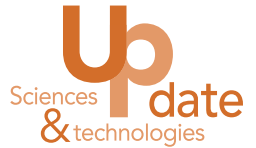

\title{
AGROFORESTERIE ET SERVICES ÉCOSYSTÉMIQUES EN ZONE TROPICALE
}

Josiane Seghieri et Jean-Michel Harmand, coordinateurs

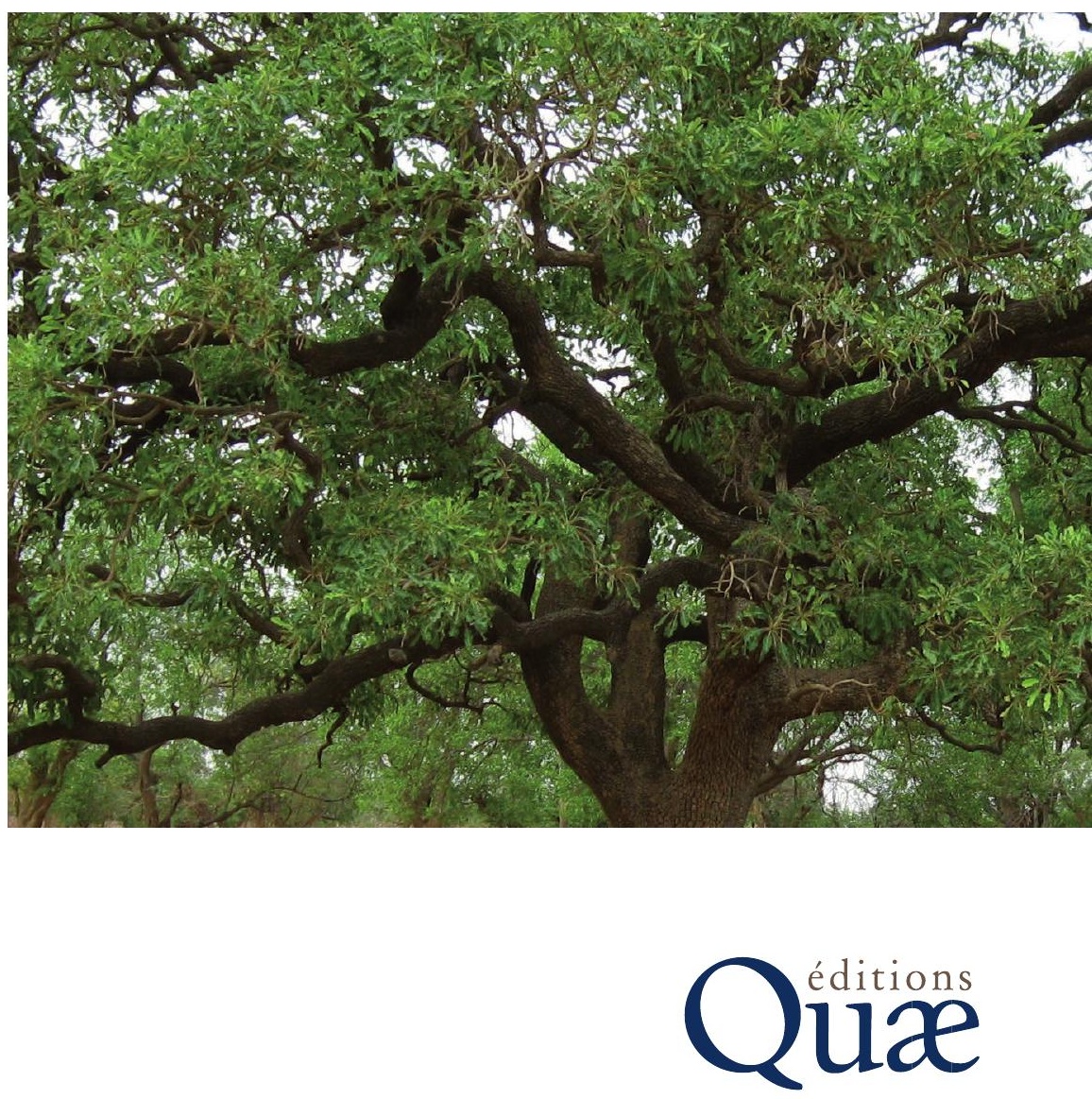




\section{Agroforesterie et services écosystémiques en zone tropicale}

Recherche de compromis entre services d'approvisionnement et autres services écosystémiques

Josiane Seghieri et Jean-Michel Harmand, coordinateurs 


\section{Collection Update Sciences \& Technologies}

Systèmes agraires et changement

climatique au Sud

H. Cochet, O. Ducourtieux,

N. Garambois, coord.

2018

Trente années d'observation

des microalgues et des toxines

d'algues sur le littoral

C. Belin, D. Soudan

2018

Stratégies des filières fromagères

sous AOP en Europe

Modes de régulation

et performance économique

P. Jeanneaux

2018

Services écosystémiques

et protection des sols

Analyses juridiques

et éclairages agronomiques

C. Hermon, coord.

2018
Paroles de chercheurs

Environnement et interdisciplinarité

E. Brun, J.-F. Ponge, J.-C. Lefeuvre

2017, $124 \mathrm{p}$.

Démanteler les barrages

pour restaurer les cours d'eau

Controverses et représentations

R. Barraud, M.-A. Germaine, coord.

2017, 260 p.

Construire des politiques

alimentaires urbaines

Concepts et démarches

C. Brand, N. Bricas, D. Conaré,

B. Daviron, J. Debru, L. Michel,

C.-T. Soulard, coord.

2017, 160 p.

Éditions Quæ

RD 10, F-78026 Versailles Cedex

(C) Quæ, 2019

ISBN (Pdf) : 978-2-7592-3059-4

ISBN Quæ (ePub) : 978-2-7592-3060-0

Cet ouvrage est sous licence CC-by-NC-ND. Vous êtes autorisé à partager - copier, distribuer et communiquer le matériel par tous moyens et sous tous formats selon les conditions suivantes :

- attribution - Vous devez créditer l'œuvre et indiquer ces informations par tous les moyens raisonnables, sans toutefois suggérer que l'éditeur et l'auteur vous soutiennent ou soutiennent la façon dont vous avez utilisé l'œuvre.

- pas d'utilisation commerciale — Vous n'êtes pas autorisé à faire un usage commercial de cette œuvre, tout ou partie du matériel la composant.

- pas de modifications - Dans le cas où vous effectuez un remix, que vous transformez, ou créez à partir du matériel composant l'œuvre originale, vous n'êtes pas autorisé à distribuer ou mettre à disposition l'œuvre modifiée.

- pas de restrictions complémentaires — Vous n'êtes pas autorisé à appliquer des conditions légales ou des mesures techniques qui restreindraient légalement autrui à utiliser l'œuvre dans les conditions décrites par la licence.

https://creativecommons.org/licenses/by-nc-nd/2.0/fr/ 


\section{Table des matières}

Préface

Préambule

Remerciements

Introduction

Jean-Michel Harmand et Josiane Seghieri

PARTIE 1.

LES SYSTÈMES AGROFORESTIERS À BASE DE CULTURES PÉRENNES : CAFÉIERS ET CACAOYERS

A - Systèmes à base de caféiers

Chapitre 1. Pressions environnementalistes et stratégies des agriculteurs dans les systèmes agroforestiers caféiers au Costa Rica et au Nicaragua.

Sibelet N., Fréguin-Gresh S., Le Coq J.-F., Gutiérrez Montes I., Laffourcade R., Dhorne S., Dorgans-Cadilhac J., Baranger M. et Constanty M.

Chapitre 2. Suivi des services écosystémiques dans un observatoire de caféiers agroforestiers. Applications pour la filière du café

Roupsard O., Allinne C., Van den Meersche K., Vaast P., Rapidel B., Avelino J., Jourdan C., Le Maire G., Bonnefond J.-M., Harmand J.-M., Dauzat J., Albrecht A., Chevallier T., Barthès B., Clément-Vidal A., Gomez-Delgado F., Charbonnier F., Benegas L., Welsh K., Kinoshita R., Vézy R., Pérez-Molina J.-P., Kim J., Taugourdeau S., Defrenet E., Nespoulous J., Rançon F., Guidat F., Cambou A., Soma M., Mages C., Schnabel F., Prieto I., Picart D., Duthoit M., Rocheteau A., Do F., de Melo Virginio Filho E., Moussa R., Le Bissonnais Y., Valentin C., Sanchez-Murillo R., Roumet C., Stokes A., Vierling L., Eitel J., Dreyer E., Saint-André L., Malmer A., Loustau D., Isaac M., Martin A., Priemé A., Eberling B., Madsen M., Robelo A., Robelo D., Borgonovo C., Lehner P., Ramirez G., Jara M., Acuña Vargas R., Barquero A., Fonseca C. et Gay F.

Chapitre 3. Régulation des bioagresseurs du caféier par le couvert arboré au Costa Rica ...... 53 Allinne C., Boudrot A., De Melo E., Granados E., Merle I., Pico J., Vonthron S. et Avelino J. 
Chapitre 4. Simulation participative pour explorer le rôle des politiques sur la production de services environnementaux au Costa Rica et au Nicaragua. 63 Le Coq J.-F., Bonifazi M., Aucante M., Ferrand N., Fréguin-Gresh S., Rapidel B. et Sibelet N.

$$
\text { B - Systèmes à base de cacaoyers }
$$

Chapitre 5. Les cacaoyères agroforestières au Centre et au Sud du Cameroun : diversité et dynamique

Michel I., Carrière S.-M., Manga Essouma F., Bihina M.A., Blanchet A., Moisy C., Ngono F. et Levang $P$.

Chapitre 6. Compromis entre séquestration de carbone, conservation et productivité dans les systèmes agroforestiers à base de cacaoyers au Centre-Cameroun .

Saj S., Jagoret P., Mvondo Sakouma K., Essobo J.-D., Bouambi E. et Todem-Ngogue H.

Chapitre 7. Structures spatiales et régulation des bioagresseurs des agroforêts à cacaoyers au Cameroun et au Costa Rica

Ngo Bieng M.A., Gidoin C. et Ten Hoopen G.M.

\section{PARTIE 2 \\ LES PARCS AGROFORESTIERS ARBORES ET ARBUSTIFS À BASE DE CUltures ANNUELles EN AFRIQUe DE L'OUeST}

$$
\text { A - Les parcs arborés }
$$

Chapitre 8. La crise du parc arboré à karité : exemple de Djougou au Bénin

Bidou J.-É., Koukpéré A. et Droy I.

Chapitre 9. Consommation en eau d'une espèce agroforestière en zone soudanienne

Awessou Kohomlan G.-B., Peugeot C., Agbossou E. et Seghieri J.

Chapitre 10. Impacts du karité sur les ressources du sol et la production d'une culture de maïs associée dans un parc agroforestier soudanien du Nord-Est du Bénin

Clermont-Dauphin C., Séguis L., Velluet C., Degbé M., Cournac L. et Seghieri J.

$$
\text { B - Les parcs arbustifs }
$$

Chapitre 11. La régénération naturelle assistée dans le bassin arachidier du Sénégal, une alternative pour réduire la pauvreté en milieu rural.

Sanogo D., Camara Baba A., Diatta Y., Coly L., Diop M., Badji M. et Binam J.-N.

Chapitre 12. Les cultures vivrières associées aux arbustes natifs :

un modèle adapté au climat sahélien

Chapuis-Lardy L., Badiane Ndour N.Y., Assigbetse K., Diédhiou I., Balaya R., Cournac L.,

Founoune-Mboup H., Mc Spadden Gardener B., Ghezzehei T., Jourdan C., Bright M.B., Bogie N., Debenport S., Delay C., Diakhaté S., Sambou D.M. et Dick R.P.

Chapitre 13. Contribution des arbustes au fonctionnement hydrique et carboné des parcs agroforestiers à Guiera senegalensis J.F. Gmel : observations et modélisation

Issoufou Bil-Assanou H., Demarty J., Cappelaere B., Allies A., Velluet C., Maïnassara I., Ö̈ M., Chazarin J.-P., Moussa Moumouni R. et Seghieri J. 


\section{PARTIE 3 \\ EXEMPLES D'ANALYSES TRANSVERSALES AU REGARD DE LA DIVERSITÉ \\ DES SYSTÈMES AGROFORESTIERS TROPICAUX}

Chapitre 14. Déterminants socio-économiques des dynamiques

des systèmes agroforestiers

Pédelahore P., Droy I., Bidou J.-E., Freguin-Gresh S., Sibelet N. et Le Coq J.-F.

Chapitre 15. Régulation des bioagresseurs des cultures

dans les systèmes agroforestiers tropicaux, revue des approches

Bagny Beilhe L., Allinne C., Avelino J., Babin R., Brévault T., Gidoin C., Ngo Bieng M.A., Motisi N., Soti V. et Ten Hoopen G.M.

Liste des auteurs 



\section{Préface}

La publication de cet ouvrage collectif «Agroforesterie et services écosystémiques en zone tropicale» nous paraît essentielle pour alerter les décideurs sur l'importance et l'urgence d'encourager et d'accompagner le développement de l'agroforesterie en milieu tropical afin de résoudre durablement les problèmes de sécurité alimentaire, environnementaux et économiques que rencontrent quotidiennement les populations rurales.

Cet ouvrage nous conforte et corrobore notre démarche naturaliste et empirique de terrain. En effet, l'Association pour la promotion des arbres fertilitaires, de l'agroforesterie et de la foresterie (Apaf) a comme vocation principale de former les paysannes et paysans à l'implantation d'arbres fertilitaires (principalement de la famille des légumineuses) dans leurs champs cultivés. Depuis les années 1990, l'Apaf a favorisé l'association de ces arbres aux cacaoyers, caféiers, palmiers, bananiers, arbres fruitiers et aux cultures vivrières dans la plupart des pays d'Afrique de l'Ouest et du Centre (environ 8 millions d'arbres implantés). Une grande partie de ces systèmes est traitées par les auteurs de cet ouvrage. Nous les félicitons pour l'ensemble des analyses exposées et espérons qu'ils aient, dans l'avenir, les moyens nécessaires pour poursuivre leurs recherches dans ce domaine.

Face au changement climatique, à la perte de biodiversité, à la déforestation, à l'appauvrissement des sols et à la dégradation des conditions de vie des populations rurales des zones tropicales, l'association des scientifiques et des acteurs de la société civile telle que l'Apaf nous paraît primordiale pour permettre la diffusion à grande échelle de techniques agroforestières innovantes en milieu paysan. Laccroissement de la résilience des systèmes agricoles, des paysages et des moyens d'existence des populations face aux changements globaux (climatiques, démographiques, économiques) en dépend.

Puisse cet ouvrage participer à la promotion de l'agroforesterie tant auprès des scientifiques que des agents de développement, des bailleurs et du grand public.

Pour l'Apaf,

Pascal Humbert, Président d'Apaf internationale et Bruno Devresse, Directeur exécutif d'Apaf internationale 



\section{Préambule}

Pratiques traditionnelles des agricultures familiales dans de nombreuses régions tropicales et tempérées, les systèmes agroforestiers ont été «redécouverts»par les organismes de recherche nationaux et internationaux il y a quelques décennies en Afrique comme en Asie ou en Amérique latine.

Louvrage coordonné par le Cirad et l'IRD, tous deux organismes de recherche pour le développement, examine des projets menés dans des contextes variés et avec de nombreux partenaires. Il combine des approches scientifiques et des disciplines complémentaires afin d'analyser le fonctionnement de différents systèmes agroforestiers, avec un intérêt plus particulier sur les systèmes agroforestiers à caféiers et cacaoyers et les parcs agroforestiers des régions sèches africaines.

Les études présentées font bien ressortir la multifonctionnalité et la diversité des biens et des services produits par les systèmes agroforestiers analysés. Elles replacent ces systèmes dans des trajectoires d'innovation et d'adaptation portées par celles et ceux qui les construisent et les gèrent.

Les différents chapitres de l'ouvrage montrent en quoi ces systèmes agroforestiers, développés par les paysans pour répondre à leurs besoins et contraintes locales spécifiques, se révèlent plus en phase avec les visées des grandes conventions internationales, notamment celles portant sur la biodiversité, le changement climatique et la désertification. Ces systèmes peuvent contribuer à plusieurs des 17 «Objectifs du développement durable», en particulier aux objectifs «Faim zéro (2)», «Consommation et production responsables (12)», «Lutte contre les changements climatiques (13)», et «Vie terrestre (15)».

Louvrage rappelle enfin l'apport nécessaire de recherches pluridisciplinaires et multipartenariales, contributions majeures pour un développement par la recherche au service des sociétés et de leurs populations.

Bernard Mallet, ancien directeur du département des forêts du Cirad, actuellement rattaché à la direction générale déléguée recherche et stratégie 



\section{Remerciements}

Nos remerciements s'adressent en priorité aux auteurs et co-auteurs, dont on trouvera la liste en fin de volume. Nous remercions également l'équipe de coordination éditoriale pour son appui constant.

Nous remercions particulièrement les relecteurs des articles qui ont permis la réalisation de cet ouvrage : Clémentine Allinne, Tiphaine Chevalier, Hubert de Foresta, Jean-Michel Harmand, Patrick Jagoret, Patrice Levang, Maud Loireau, Olivier Planchon, Josiane Seghieri, Alain Ratnadass, Olivier Roupsard, Georges Serpantié et Philippe Thaler.

Nous tenons à remercier également tous les chercheurs, les techniciens, les acteurs de terrain, les agriculteurs, etc., impliqués dans la réalisation des études qui ont abouti à la publication de cet ouvrage, ainsi que les personnes et les organismes qui ont contribué à leur financement. 



\title{
Introduction
}

\author{
Jean-Michel Harmand et Josiane Seghieri
}

\section{W Contexte}

Lagroforesterie est un système de gestion des ressources naturelles qui, grâce à l'intégration des arbres dans les exploitations agricoles et dans le paysage agricole, permet de diversifier et soutenir la production et d'accroître la résilience des paysages et des moyens d'existence en milieu rural. Lagroforesterie compte parmi les pratiques d'exploitation des terres qui existent depuis des siècles sur tous les continents. De très nombreux agriculteurs des pays tropicaux en vivent. Selon Lundgren et Raintree (1982), la gestion des systèmes agroforestiers repose sur la recherche de la durabilité d'une production diversifiée en valorisant les interactions écologiques, économiques et sociales existant entre les composantes de ces systèmes. Lagroforesterie constitue l'une des solutions d'utilisation durable de ressources naturelles limitées et d'adaptation aux changements globaux démographiques, économiques et climatiques. En multipliant les strates végétales, cette pratique permet d'augmenter la diversité des espèces, des habitats, des niches écologiques, des modes d'occupation de l'espace, des fonctions et des fonctionnements écologiques. Les rythmes d'utilisation des ressources environnementales sont aussi accrus car ces dispositifs améliorent la captation, la fixation, l'utilisation et le recyclage in situ de ces ressources.

Cependant, il ne faut pas sous-estimer la complexité de la structure et du fonctionnement des systèmes agroforestiers, en particulier celle des interactions entre les arbres et les cultures associées. En effet, cette complexité repose sur les principes de fonctionnement des écosystèmes naturels, notamment forestiers. La viabilité de ces écosystèmes dépend donc de la connaissance de ces principes et de la maîtrise des facteurs qui contrôlent leurs productions multiples. Moins consommateurs d'intrants et d'énergie que les systèmes agricoles conventionnels, ces systèmes combinent des 
services écologiques et des productions diverses qui contribuent à la sécurité alimentaire, économique et foncière des exploitants, notamment les petits exploitants des pays tropicaux. Les systèmes agricoles, quels qu'ils soient, sont sources de services écosystémiques, mais aussi de «disservices» tels que la perte de biodiversité, la pollution agrochimique, l'érosion des sols, l'émissions de gaz à effet de serre (Power, 2010). Comparativement aux systèmes agricoles conventionnels, les systèmes agroforestiers déplacent cet équilibre fonctionnel vers la fourniture de services. Dans les associations agroforestières, les services d'approvisionnement (productions) dépendent, entre autres, de l'impact de la strate arborée sur le rendement de la culture de base et des productions complémentaires multiples fournies par cette strate arborée. Les services de régulation - en particulier ceux du cycle et de la qualité de l'eau, du climat à travers le contrôle des émissions de gaz à effet de serre et le stockage de carbone - y sont favorisés. Enfin, selon leur composition, leur structure et leur gestion, les systèmes agroforestiers contribuent de façon plus ou moins importante aux services de support des services précédents. Parmi ces services de support, on peut citer : la production primaire, le contrôle des bioagresseurs, la conservation des sols, les cycles des nutriments et la préservation de la biodiversité.

Lagroforesterie contribue ainsi à la mise en place ou au maintien de paysages multifonctionnels résilients face au changement climatique. En diversifiant les productions et les revenus, les systèmes agroforestiers réduisent la vulnérabilité des agriculteurs face à la volatilité des prix des denrées. De ce fait, ils sont une composante essentielle du patrimoine des ménages transmis de génération en génération. La sécurisation foncière est d'ailleurs un préalable à leur maintien sur le long terme et à la possibilité de rétribution des services environnementaux qu'ils fournissent.

Lagroforesterie constitue l'une des solutions potentielles à mettre en œuvre pour faire face au défi de l'intensification écologique des agroécosystèmes en permettant de produire plus et mieux avec peu d'intrants, tout en s'adaptant au changement climatique. Cependant, le potentiel d'amélioration de la production agricole via les systèmes agroforestiers est encore peu exploré, notamment pour optimiser leur fonctionnement sur le long terme.

\section{" Objectif de l'ouvrage}

Cet ouvrage a pour ambition de documenter l'évaluation des performances agronomiques, environnementales et économiques des systèmes agroforestiers tropicaux. Plus particulièrement, sont étudiés les impacts de la structure, de la composition et de la gestion de ces systèmes agroforestiers sur les services écosystémiques fournis (approvisionnement et autres services). Sont documentés les interactions, compromis et synergies entre ces services afin de proposer des scénarios réalistes d'amélioration de ces systèmes. Une analyse des déterminants socio-économiques des dynamiques des systèmes agroforestiers est également présentée pour les différents cas d'étude.

Les études ont été menées par des chercheurs du Cirad, de l'IRD et des principales institutions partenaires des pays en développement en zone tropicale à partir de différents projets et dans le cadre de dispositifs en partenariat (DP) «Agroforesterie Cameroun», «Systèmes agroforestiers à base de cultures pérennes en Amérique 
centrale» (AFS-PC). Elles ont été également menées au sein d'observatoires collaboratifs multidisciplinaires de projets comme :

- Coffee-flux (Observatory for monitoring and modeling carbon, nutrients, water and sediment ecosystem services in coffee agroforestry systems) au Costa Rica;

- Amma-Catch (Analyse multidisciplinaire de la mousson africaine - Couplage de l'atmosphère tropicale et du cycle hydrologique) en Afrique de l'Ouest;

- le projet Cirad-IRD sur la «Recherche de compromis entre productions et autres services écosystémiques fournis par les systèmes agroforestiers tropicaux (Safse, 2012-2015);

- les projets de la National science foundation (NSF). Partnerships for international research and education (PIRE, 2001-2008 et PIRE 2010-2017);

- des projets de l'Agence nationale française pour la recherche (ANR), «Élevageclimat-société » (Eclis, 2009-2012; Ecosfix, 2011-2014), «Environmental and social changes in Africa: past, present and future» (Escape, 2011-2015), «Modélisation pour l'accompagnement des acteurs, vers l'adaptation des couverts pérennes ou agroforestiers aux changements globaux» (Macacc 2014-2016);

- les projets européens «Mise en relation des services environnementaux et de la valeur marchande de l'agroforesterie caféière» (Cafnet, 2007-2010), «Lagroforesterie au service de la sécurité alimentaire» (AFS4Food 2012-2015).

Les activités concernées s'appuient sur des dispositifs de recherche localisés dans des situations particulièrement contrastées.

\section{Structure de l'ouvrage}

La première partie de l'ouvrage traite des systèmes agroforestiers à base de cultures pérennes - caféiers (chapitres 1 à 4) et cacaoyers (chapitres 5 à 7) — très répandus dans les zones tropicales humides. La deuxième partie de l'ouvrage concerne les parcs agroforestiers arborés (chapitres 8 à 10) et arbustifs (chapitres 11 à 13) à base de cultures annuelles dans les zones semi-arides d'Afrique de l'Ouest. La troisième partie de l'ouvrage donne deux exemples d'analyse transversale au regard de la diversité des systèmes agroforestiers : les déterminants socio-économiques de leur dynamique (chapitre 14) et la régulation des bioagresseurs (chapitre 15).

Une première thématique porte sur les stratégies et impacts des acteurs sur la structure et la dynamique spatiale des systèmes agroforestiers au regard de leur diversité et des services écosystémiques qu'ils fournissent (chapitres 1, 4, 5, 8, 11 et 15) :

- le chapitre 1 (Sibelet et al.) compare les impacts des pressions environnementalistes au Costa Rica et au Nicaragua. Au Costa Rica où les systèmes de production et niveaux socio-économiques sont relativement homogènes, le discours environnementaliste des agriculteurs est souvent en décalage avec leurs pratiques. Au Nicaragua les pressions environnementalistes se heurtent à une grande diversité de situations et aux priorités de survie des populations;

- le chapitre 4 (Le Coq et al.) propose une approche participative et prospective conçue et mise en œuvre dans deux zones de production de café, au Costa Rica et au Nicaragua, pour tester l'intérêt de certains instruments incitatifs de pratiques agricoles pourvoyeuses de services environnementaux; 
- le chapitre 5 (Michel et al.) montre l'évolution des structures des plantations de cacaoyers au Centre et au Sud du Cameroun et dans différentes situations biogéographiques. Ainsi, des agroforêts cacaoyères multistrates semblables au modèle ancien se maintiennent, se transforment en partie ou continuent de s'étendre. Parallèlement, des structures plus simples se développent. Elles sont essentiellement portées par de nouveaux investisseurs dans la cacaoculture de rente;

- le chapitre 8 (Bidou et al.) montre que les dynamiques en cours peuvent contribuer à expliquer comment, malgré une demande importante en karité sur le marché international, beaucoup de parcs à karité sont en voie de dégradation au Bénin. Pour parvenir à cette démonstration, les auteurs lient les évolutions du parc à karité aux changements sociaux, en particulier à la répartition de la gestion de la ressource et des terres entre les hommes et les femmes;

- le chapitre 11 (Sanogo et al.) évalue les avantages socio-économiques que les ménages agricoles tirent de la régénération naturelle assistée dans deux zones agroclimatiques du Sénégal : le centre-nord et le centre-sud du bassin arachidier. Il analyse également les contraintes naturelles, anthropiques et institutionnelles de l'adoption de cette régénération naturelle assistée;

- le chapitre 14 (Pedelahore et al.) montre qu'au-delà des spécificités de chacune des neuf situations analysées au Bénin, au Cameroun, au Costa Rica, au Kenya et au Nicaragua, il existe des récurrences dans les dynamiques observées et dans leurs déterminants socio-économiques établis en fonction de quatre grandes classes : les stratégies des exploitants agricoles, la disponibilité en ressources, les caractéristiques des marchés et les régulations et les appuis en vigueur dans la zone étudiée. Généralement, les grands producteurs disposant de capitaux réduisent la couverture arborée pour privilégier la culture principale. Les petits producteurs développent des services agroforestiers variés permettant la diversification des produits vivriers et marchands, ainsi qu'une réduction des risques alimentaires et commerciaux.

La deuxième thématique porte sur les interactions et les compromis existant entre productions, conservation de la biodiversité végétale, séquestration du carbone et régulation des flux hydriques au sein des services agroforestiers (chapitres 2, 6, 9 et 13) :

- le chapitre 2 (Roupsard et al.) synthétise un grand nombre de résultats multidisciplinaires obtenus sur le long terme (observatoire Coffee-flux) dans une ferme caféière sous légumineuse arborée au Costa Rica. Il montre que la gestion du caféier pourrait être améliorée par des tailles appropriées, l'ajustement de l'ombrage et des apports d'intrants. On peut ainsi obtenir une production plus efficiente en termes d'utilisation de l'énergie, de l'eau et des nutriments, en particulier de l'azote. Des propositions d'amélioration de l'évaluation des bilans de carbone des fermes caféières sont également issues de ces résultats;

- le chapitre 6 (Saj et al.) analyse les compromis existant entre les trois services - production de cacao, conservation des espèces ligneuses et stockage de carbone dans les systèmes agroforestiers du Centre du Cameroun — en soulignant la synergie entre les deux services de régulation et l'antagonisme de ces derniers avec le rendement accessible en cacao. Néanmoins, se dégagent des compromis intéressants entre des niveaux moyens à élevés de diversité végétale et de stockage de carbone, ainsi que des rendements en cacao performants lorsque des pratiques adéquates sont adoptées; 
- le chapitre 9 (Awessou et al.) montre qu'en considérant seulement la transpiration du couvert du karité estimée dans une jachère de dix ans en zone soudanienne, la marge de densification des arbres est potentiellement élevée. En effet, la transpiration reste très faible par rapport aux précipitations et à la demande évaporative annuelles (moins de 2\%);

- le chapitre 13 (Issoufou et al.) démontre, dans un site sahélien au Niger, la plus grande contribution à l'évapotranspiration réelle et la plus grande capacité de production des rejets de Guiera senegalensis rabattus chaque année en champs par rapport aux arbustes matures de la même espèce en jachère.

La troisième thématique traite du rôle des couverts arborés dans la régulation des bioagresseurs des cultures associées (chapitres 3, 7 et 15):

- le chapitre 3 (Allinne et al.) analyse, dans des systèmes agroforestiers à base de caféiers au Costa-Rica, les effets des interactions entre l'ombrage et les conditions microclimatiques, entre l'ombrage et le cycle de vie d'un agent pathogène (la rouille du caféier), entre des bioagresseurs du complexe parasitaire et entre des auxiliaires et des bioagresseurs. Il montre aussi qu'un ombrage optimal peut réduire l'impact total du cortège de bioagresseurs;

- le chapitre 7 (Ngo Bienget al.) démontre, au Costa Rica et au Cameroun, comment la répartition spatiale des individus (arbres et cacaoyers) influence l'intensité d'attaque de bioagresseurs du cacaoyer (moniliose au Costa Rica et mirides au Cameroun). Comparativement à une distribution agrégée des arbres forestiers, des distributions régulières ou aléatoires réduisent les intensités d'attaque des bioagresseurs;

- le chapitre 15 (Bagny et al.) synthétise un ensemble d'études sur les mécanismes de régulation des maladies et des ravageurs menées dans des systèmes agroforestiers à base de caféiers, de cacaoyers et de mil au Cameroun, au Costa Rica, au Kenya et au Sénégal. Il met en évidence l'effet de la composition et de l'organisation spatiale de la biodiversité associée sur les bioagresseurs, l'effet de l'ombrage sur le développement des bioagresseurs, l'effet de la biodiversité végétale du couvert, aux échelles parcelle et paysage, sur les communautés d'ennemis naturels des bioagresseurs et leur efficacité à réguler leurs populations.

La quatrième thématique traite des interactions entre les arbres ou les arbustes et les cultures associées au regard des ressources du sol, de la production et du rendement de la culture (chapitres 10 et 12 ) :

- le chapitre 10 (Clermont-Dauphin et al.) évalue, dans un système agroforestier au Nord-Est du Bénin, les effets en apparence paradoxaux du karité sur les ressources du sol (eau, nutriments et carbone) et sur le rendement de la culture de maïs associée. Cette évaluation met en évidence un plus faible rendement de la culture sous l'arbre et un effet positif de l'arbre sur la matière organique du sol à l'échelle de la parcelle;

- le chapitre 12 (Lardy et al.) dresse le bilan de quinze années de recherches sur le fonctionnement des sols des systèmes agroforestiers sahéliens au Sénégal associant milarachide et arbustes natifs (Guiera senegalensis ou Piliostigma reticulatum). Ces travaux démontrent l'effet significatif d'«îlots de fertilité» autour des arbustes. Ces îlots sont propices au développement de communautés microbiennes (bactéries et champignons) impliquées dans la fourniture d'éléments nutritifs et la régulation de certains bioagresseurs (nématodes phytophages) au bénéfice d'une meilleure croissance et d'un meilleur rendement de la culture associée encore améliorable par une gestion appropriée. 
En résumé, les différentes études présentées dans cet ouvrage quantifient et qualifient la contribution positive des strates arborées des systèmes agroforestiers à un grand nombre de services écosystémiques tels que la diversification des productions, l'amélioration du microclimat, la qualité et la santé des sols, le stockage de carbone, la régulation des bioagresseurs des cultures et la conservation des espèces. Aussi, se dégagent de ces études des possibilités de compromis intéressants et de fortes synergies entre le rendement de la culture de base et les autres services écosystémiques. Ces compromis et ces synergies plaident en faveur du maintien et d'une gestion adaptée des couverts arborés dans les systèmes agroforestiers pour une production durable.

Néanmoins, les études sur la dynamique temporelle et spatiale des systèmes agroforestiers mettent en évidence des tendances à la réduction des couverts liée à des pratiques d'intensification et à des changements d'acteurs ruraux, conduisant ainsi à des pertes de services écosystémiques et de résilience de ces systèmes. Des politiques publiques favorisant l'agroforesterie sous des formes prennant en compte les priorités des populations concernées pourraient s'appuyer sur différents mécanismes tels que les incitations réglementaires, la rétribution de services environnementaux, l'appui et le conseil ou encore la labéllisation des produits. Aussi, la structuration et la sécurisation des filières concernées par les systèmes agroforestiers permettraient également d'éviter leur conversion totale en monocultures de rente, provoquée par des opportunités nouvelles offertes par les marchés.

\section{Bibliographie}

Lundgren B.O., Raintree J.B., 1982. Sustained agroforestry. In: Nestel B., (ed). Agricultural research for development: potentials and challenges in Asia. The Hague: Isnar, 37-49.

Power A.G., 2010. Ecosystem services and agriculture: trade-offs and synergies. Philosophical transactions of the royal society B: Biological sciences, 365: 2959-2971. 
Partie 1

Les systèmes agroforestiers à base de cultures pérennes : caféiers et cacaoyers 

A - Systèmes à base de caféiers 



\title{
Chapitre 1 \\ Pressions environnementalistes et stratégies des agriculteurs dans les systèmes agroforestiers caféiers au Costa Rica et au Nicaragua
}

\author{
Sibelet N., Fréguin-Gresh S., Le Coq J.-F., Gutiérrez Montes I., \\ Laffourcade R., Dhorne S., Dorgans-Cadilhac J., \\ Baranger M. ET Constanty M.
}

Résumé. L'agroforesterie est souvent citée pour sa capacité à concilier la préservation de l'environnement, tout en assurant un revenu et une production satisfaisante aux agriculteurs. Le présent chapitre traite des pressions environnementalistes sur l'agroforesterie au Nicaragua et au Costa Rica, où la question de préservation de l'environnement concerne notamment la biodiversité, l'érosion des sols, la ressource en eau et la couverture arborée. L'objectif de notre recherche est de comprendre comment les pressions environnementalistes influent sur les stratégies des agriculteurs. Ces pressions sont assez similaires dans les deux pays, mais les résultats sont distincts. Au Costa Rica, l'homogénéité et la force des discours ont produit une idéologie environnementaliste globale qui s'applique à une situation relativement homogène en termes de systèmes de production et de niveau socioéconomique. Néanmoins un décalage existe encore entre le discours des agriculteurs et leurs pratiques. Au Nicaragua, les multiples pressions environnementalistes se heurtent à une grande diversité de situations des systèmes de production et du niveau socio-économique, ainsi qu'aux priorités relevant de la survie des populations les plus précaires.

\begin{abstract}
Agroforestry is often cited for its capacity to preserve the environment while also providing sufficient production and income for farmers. This chapter looks at environmentalist pressures in Nicaragua and Costa Rica, both subjected to questions regarding environmental conservation notably in terms of biodiversity, soil erosion, water conservation and forest cover. The objective of our research is to understand how environmentalist pressures influence farmers' strategies. Environmentalist pressures are similar in both countries but their effects are distinct. In Costa Rica, the homogeneity and the strength of the discourse have resulted in a comprehensive environmentalist
\end{abstract}


ideology that applies to a relatively homogenous situation in terms of farming systems and socio-economic level, nonetheless a discrepancy still exists between farmers' discourses and their practices. In Nicaragua, multiple environmentalist pressures apply to a diversity of situations regarding farming systems, socio-economic level and the priorities relevant to the survival of its most precarious inhabitants.

\section{Introduction}

Les systèmes agroforestiers sont souvent cités pour leur capacité à concilier la préservation de l'environnement et l'assurance d'un revenu tout en assurant une production satisfaisante pour les agriculteurs. Dans le cadre du projet Safse (Compromis entre production et autres services écosytémiques fournis par les systèmes agroforestiers, Cirad/IRD), nous avons contribué à comprendre si cette conciliation est effective et dans quelles conditions.

Une pression environnementaliste est «une pression émise par une entité sur une autre entité sous la forme d'un discours, d'une argumentation ou d'un objectif afin de préserver l'environnement» (Sibelet, 2015).

Les pressions environnementalistes sont traitées dans deux régions, au Nicaragua et au Costa Rica, elles sont caractérisées par la présence de systèmes agroforestiers et d'enjeux environnementaux. Ces enjeux concernent :

- la perte de la biodiversité;

- la dégradation des sols, notamment par l'érosion hydrique;

- la déforestation ou diminution du nombre des arbres-hors-forêts liée au changement d'usage des sols;

- la pollution de l'eau due aux résidus de produits agrochimiques ou aux effluents de la première transformation du café;

- l'augmentation de la charge en sédiments de l'eau pouvant, par exemple, envaser une retenue d'eau de barrage hydroélectrique.

Lobjectif de ce chapitre est, d'une part, d'identifier et de caractériser les pressions environnementalistes mises en œuvre par des acteurs intervenant localement dans l'agriculture et l'environnement et, d'autre part, d'analyser comment ces pressions s'exercent sur les stratégies et les pratiques des agriculteurs.

\section{Méthodes}

\section{Sites d'étude}

Deux sites d'étude ont été choisis. Dans les deux cas, la production agricole, la préservation de l'environnement et leurs articulations sont des questions prégnantes.

\section{Site de La Dalia, Nicaragua}

Au Nicaragua, le site d'étude est localisé au sud du massif de Peñas Blancas, il inclut une réserve naturelle (Gómez et al., 2011) couverte de forêt sempervirente qui culmine à $1750 \mathrm{~m}$ d'altitude et marquée par de fortes pentes. Ce site est à l'interface de quatre communes dont El Tuma-La Dalia encore appelée La Dalia. 
La qualité agronomique des sols et le climat de type tropical humide d'altitude (2000-2500 mm de pluviométrie et $22-24^{\circ} \mathrm{C}$ de température moyenne) font de ce site un lieu d'excellence pour la production de café. Le café de cette région se distingue au niveau international par sa qualité.

La majeure partie de la population rurale de La Dalia vit de l'utilisation des ressources naturelles du massif, en particulier pour y développer une agriculture familiale (93\% des exploitations agricoles en 2011) (Cenagro, 2012). Les systèmes de production agricole en ouvre combinent des caféiers sous ombrage de densité variable et de type diversifié - ombrage composé de fruitiers et de multiples espèces d'arbres forestiers - , des cultures vivrières (maïs, haricots, racines et tubercules), du maraîchage et de l'élevage (essentiellement de bovins). Toutefois, la production agricole ne permet pas de générer des revenus suffisants pour un grand nombre de producteurs, notamment en raison d'une dotation foncière inéquitable et d'un accès différencié au marché (Arribard, 2013).

\section{Le site de Llano Bonito, Costa Rica}

La région de Los Santos, située au centre-ouest du Costa Rica, produit le tiers de la production nationale de café. Cette culture façonne le paysage de cette région. Le district de Llano Bonito est montagneux, dominé par le Cerro Dragón qui culmine à $2506 \mathrm{~m}$. Il est représentatif de la région avec des pentes de plus de $80 \%$ (Meylan et al., 2013). Les flancs de montagne sont presque entièrement couverts par des caféiers sous ombrage essentiellement d'Erythrina poeppigiana et de bananiers (Musa sp.) Dans les six villages de Llano Bonito, vivent 2500 habitants (RoseroBixby et Dow, 2012). Les 700 à 800 agriculteurs de la zone d'étude cultivent tous du caféier (Coffea arabica).

Le site de Llano Bonito, d'environ 1800 hectares, produit exclusivement de l'arabica. Les caféiculteurs sont propriétaires de petites exploitations agricoles, pour la plupart entre deux et cinq hectares, et produisent un café de haute qualité liée à l'altitude élevée de $1500 \mathrm{~m}$ en moyenne (Meylan et al., 2017). La dénomination «café Tarrazú », encore en cours de formalisation d'appellation d'origine contrôlée, est cependant déjà connue dans le monde entier et bénéficie d'une prime de qualité élevée sur le marché international. Selon les chaînes de valeur, les prix de vente en gros bénéficient d'un premium qui varie entre 40 et 100 US\$ par sac de $45,4 \mathrm{~kg}$ de café (Snider et al., 2016).

Le relief très escarpé, le type de sol et les fortes précipitations durant la saison des pluies font de l'érosion des sols un problème environnemental majeur pour la zone. La perte moyenne de sol sur la zone a été estimée à 22 tonnes/ha/an (Melendez Marin, 2010).

\section{Collecte de données}

Dans les deux sites d'étude, l'observation participative a fait partie intégrante du travail de recherche. Des entretiens de type compréhensif semi-directif ont été menés auprès de 52 membres d'institutions (agences gouvernementales et de coopération, ONG et coopératives) et de 62 producteurs au Nicaragua (Kaufmann, 
2011; Sibelet et al., 2013). Ils ont concerné 10 membres d'institutions et 89 habitants au Costa Rica. Cet échantillon embrasse la diversité des acteurs. Les principales variables étudiées ont été les pratiques institutionnelles, sociales et agricoles, les discours environnementalistes et les actions environnementales, les systèmes de culture, les systèmes de production agricole, les relations entre les acteurs exerçant des pressions environnementalistes et les stratégies des acteurs.

\section{" Résultats et discussion}

\section{Les pressions environnementalistes répondent aux enjeux environnementaux locaux}

Dans les deux sites, les acteurs publics et privés mettent en place différents types d'actions en vue de préserver l'environnement qui se traduisent par des pressions environnementalistes sur les agriculteurs. Le premier type de pression renvoie à l'application des réglementations existantes par les pouvoirs publics ou par des acteurs privés (voir lignes 1 et 2, tab. 1.1).

À La Dalia (Nicaragua), plusieurs acteurs publics ont pour mandat l'application des nombreuses règles de gestion de l'usage des sols et de l'environnement (Fréguin-Gresh et al., 2014). Ainsi, les délégués ministériels au niveau municipal ayant des compétences dans le secteur agricole et environnemental sont en charge de l'application des lois en vigueur, en particulier le ministère de l'Économie familiale, communautaire, coopérative et associative (Mefcca), l'Institut national des forêts (Inafor), l'Institut national des technologies agricoles (Inta), le ministère de l'Environnement et des Ressources naturelles (Marena), le ministère de l'Agriculture et de l'Élevage (MAG) et la mairie à travers son Unité environnementale (UAM). Lapplication de ces normes est un défi à cause du manque de ressources financières et de personnel. Lessentiel des actions de ces fonctionnaires est l'application de sanctions (amendes) lorsqu'ils constatent des infractions et des délits environnementaux (coupes d'arbres sans permis, rejets d'effluents incontrôlés). Parallèlement, des ONG tentent de faire respecter les règles d'usage des sols dans les différentes zones de la réserve naturelle. Parmi leurs actions, la mise en défens par l'achat de terres vise à conserver l'environnement en interdisant de facto la mise en culture, notamment à proximité des sources d'eau du massif situées dans la zone-cœur de la réserve.

Llano Bonito est, comme tout le reste du Costa Rica, sous le régime de nombreuses lois nationales faites pour préserver l'environnement : interdiction de certains produits phytosanitaires, interdiction de déforester (loi forestière n 7575 de 1996) et interdiction de cultiver à proximité de cours d'eau. Toutefois, si l'application des deux premières interdictions est relativement bien respectée, la loi sur la protection des cours d'eau est peu contrôlée et donc peu respectée.

Les acteurs publics et privés mettent également en œuvre des actions incitatives auprès des producteurs, qui passent par des formations et des campagnes de sensibilisation et des incitations économiques (certifications) (lignes 3 et 4 du tab. 1.1). 
Tableau 1.1. Pressions environnementalistes dans les deux régions étudiées au Nicaragua et au Costa Rica (données issues des études de Baranger et al., 2015; Dhorne, 2013; Dorgans-Cadilhac, 2013; Laffourcade, 2012)

\begin{tabular}{|c|c|c|c|}
\hline & $\begin{array}{c}\text { Pression } \\
\text { environnementaliste }\end{array}$ & Instruments & $\begin{array}{l}\text { Acteurs exerçant } \\
\text { les pressions } \\
\text { environnementalistes }\end{array}$ \\
\hline 1 & $\begin{array}{l}\text { Par des interdictions } \\
\text { de certains usages } \\
\text { du sol, en fonction } \\
\text { du zonage de } \\
\text { la réserve naturelle } \\
\text { Mise en défens } \\
\text { de terres pour } \\
\text { la conservation }\end{array}$ & $\begin{array}{l}\text { Régulation directe par } \\
\text { les autorités ayant pour mandat } \\
\text { l'aménagement du territoire et } \\
\text { la réglementation de l'usage des } \\
\text { terres dans le cadre d'une aire } \\
\text { protégée (au Nicaragua et } \\
\text { Costa Rica) } \\
\text { Achat de terres pour la mise } \\
\text { en défens (Nicaragua) }\end{array}$ & $\begin{array}{l}\text { Nicaragua : organisations } \\
\text { publiques (ministère } \\
\text { de l'Environnement - } \\
\text { Marena; ministère de } \\
\text { l'Agriculture - Mag; Institut } \\
\text { national des Forêts - Inafor) } \\
\text { et autorités locales (mairie) } \\
\text { Costa Rica : organisations } \\
\text { publiques (Minae; } \\
\text { Système national des aires } \\
\text { de conservation - Sinac) } \\
\text { ONG (Nicaragua) }\end{array}$ \\
\hline 2 & $\begin{array}{l}\text { Par l'application } \\
\text { de sanctions } \\
\text { en lien avec } \\
\text { les réglementations } \\
\text { environnementales }\end{array}$ & $\begin{array}{l}\text { Interdiction de rejeter } \\
\text { des effluents de transformation } \\
\text { du café (Nicaragua) } \\
\text { Interdiction de cultiver } \\
\text { à proximité des cours d'eau } \\
\text { (Costa Rica) }\end{array}$ & $\begin{array}{l}\text { Nicaragua : Inafor, Marena, } \\
\text { ministère de la Santé, } \\
\text { Minsa; mairie (unité } \\
\text { environnementale) } \\
\text { Costa Rica : Minae, Mag }\end{array}$ \\
\hline 3 & $\begin{array}{l}\text { Par la formation } \\
\text { et sensibilisation } \\
\text { (outils cognitifs) }\end{array}$ & $\begin{array}{l}\text { Campagnes d'information et } \\
\text { de sensibilisation } \\
\text { Formations dans le cadre } \\
\text { de programmes de l'État ou } \\
\text { de projets de développement } \\
\text { Dons de plants d'arbres }\end{array}$ & $\begin{array}{l}\text { Nicaragua : ministère } \\
\text { de l'Economie familiale, } \\
\text { Mefcca, Marena; Inafor; } \\
\text { Coopératives de café, ONG, } \\
\text { universités locales) } \\
\text { Costa Rica : Mag, Icafe, Ice, } \\
\text { Ina, Coopérative de café, }\end{array}$ \\
\hline \multirow[t]{3}{*}{4} & $\begin{array}{l}\text { Par des incitations } \\
\text { économiques }\end{array}$ & $\begin{array}{l}\text { 4.1. Paiements pour services } \\
\text { environnementaux : } \\
\text { Costa Rica : paiement pour } \\
\text { la plantation d'arbres dans } \\
\text { des systèmes agroforestiers } \\
\text { Nicaragua : crédits verts } \\
(1)\end{array}$ & $\begin{array}{l}\text { Costa Rica : Minae, Fonafifo } \\
\text { mais pas dans la zone de } \\
\text { Llano Bonito. } \\
\text { Nicaragua : ONG }\end{array}$ \\
\hline & & $\begin{array}{l}\text { 4.2. Transferts directs de projets } \\
\text { ou fourniture d'intrants à crédit } \\
\text { sous conditions d'adoption } \\
\text { de pratiques plus favorable } \\
\text { à l'environnement }\end{array}$ & $\begin{array}{l}\text { Nicaragua : Exportateurs } \\
\text { de café, Mefcca, } \\
\text { coopératives, exportateurs } \\
\text { de café, entreprises } \\
\text { de micro-crédit } \\
\text { Costa Rica : non }\end{array}$ \\
\hline & & $\begin{array}{l}\text { 4.3. Certification socio- } \\
\text { environnementale (commerce } \\
\text { équitable, Rainforest alliance, } \\
\text { Biologique, Cafe practices) }\end{array}$ & $\begin{array}{l}\text { Costa Rica et Nicaragua } \\
\text { (encore rare) : certificateurs, } \\
\text { exportateurs de café, } \\
\text { coopératives de café }\end{array}$ \\
\hline
\end{tabular}

(1) Crédits verts : taux d'intérêts bonifiés pour certaines pratiques 


\section{À La Dalia (Nicaragua)}

À La Dalia (Nicaragua), ces actions incitatives sont essentiellement des campagnes d'information et de sensibilisation développées dans le cadre de programmes publics ou de projets privés mis en œuvre par des coopératives de production, des organisations internationales de coopération technique ou des ONG. Elles prennent la forme d'«écoles techniques de campagne» lorsqu'elles se combinent à des formations techniques. Ces campagnes peuvent s'organiser au niveau de bassins versants pollués ou déboisés pour restaurer l'écosystème ou prévenir de nouvelles infractions environnementales.

D'autres actions incitatives existent, parmi lesquelles des dons de plants d'arbres que l'Inafor et l'unité environnementale de la Mairie distribuent aux producteurs pour installer des arbres en bordure de rivière. Certains exportateurs de café proposent des prix incitatifs aux producteurs capables de respecter certains cahiers des charges. D'autres producteurs réussissent à faire certifier individuellement leur production (Rainforest, UTZ, Café Practices strategic supplier et Starbucks) en respectant ainsi des cahiers des charges ayant une forte composante environnementale. Ils peuvent alors exporter leur café à des prix plus avantageux pour eux.

En dehors de la provision de «crédits verts» conditionnés par le respect de certaines pratiques culturales, d'autres acteurs privés ont, par leurs actions, des effets adverses sur la préservation de l'environnement. C'est le cas notamment des agro-exportateurs et des caisses de micro-crédits qui, en facilitant l'accès aux intrants agrochimiques des producteurs, favorisent une utilisation parfois incontrôlée de certains produits polluants. C'est le cas notamment d'un insecticide pourtant interdit : l'endosulfan.

\section{À Llano Bonito (Costa Rica)}

À Llano Bonito (Costa Rica), le discours environnementaliste est omniprésent dans toutes les organisations locales, alors qu'elles n'ont pas d'intérêt direct dans la préservation de l'environnement. Les organisations alignent leurs discours sur l'image «verte» que le Costa Rica s'est construite. Les organisations publiques et les acteurs privés exercent des pressions environnementalistes par un discours qui privilégie un contact régulier et un climat de confiance avec les agriculteurs (Constanty, 2015; Dhorne, 2013; Laffourcade, 2012).

Ainsi, la coopérative Llano Bonito est l'organisation la plus liée aux agriculteurs. Linstitut du café (Icafe) et l'institut national d'apprentissage (Ina) s'appuient tous deux sur le canal de communication privilégié que représente cette coopérative pour transmettre recommandations, cours et documents.

La coopérative Llano Bonito et Volcafé, les deux principaux transformateurs de café de la zone, ont des certifications telles que Starbucks ${ }^{\circledR}$ Cafe practices et Nespresso AAA. Les organismes certificateurs influencent, par leurs exigences, les discours de ces deux transformateurs à destination des caféiculteurs.

LInstitut costaricien de l'électricité (ICE) a un barrage en aval des terroirs caféiers de Llano Bonito. Il préconise une caféiculture limitant l'érosion des sols pour éviter l'envasement de la retenue d'eau. Outre une collaboration avec l'ICE, le MAG a une stratégie d'appui aux activités complémentaires : l'élevage bovin, la culture d'avocat et l'apiculture. 
Par ailleurs, si le Costa Rica dispose d'un programme national de paiement pour services environnementaux pour la préservation des ressources arborées (forêts et arbres hors-forêts), celui-ci est peu mis en œuvre dans la zone d'étude.

\title{
Exploitations agricoles et systèmes agroforestiers caféicoles au Nicaragua
}

\begin{abstract}
À La Dalia (Nicaragua), les systèmes agroforestiers caféicoles sont concentrés dans la partie ouest, centrale et nord du territoire. Quelques résidus de forêts primaires sont présents dans la réserve naturelle située au nord de La Dalia. Sur le reste du territoire, alternent des zones de pâturages, des zones de cultures vivrières (maïs, haricots et légumes) et des systèmes agroforestiers (Arribard, 2013). Les systèmes agroforestiers sont diversifiés. Leur gestion est hétérogène compte tenu des écarts de niveaux socio-économiques (revenus, accès au foncier) entre les exploitations. Celles-ci peuvent être classées en exploitations familiales, exploitations patronales et haciendas, catégories subdivisées en six types (Baranger et al., 2015).
\end{abstract}

\section{Les exploitations familiales}

Type 1. Les «salariés agricoles ayant une petite parcelle de cultures vivrières» se distinguent car ils travaillent comme journaliers agricoles ou salariés permanents dans la région. De fait, ils dédient peu de temps à l'agriculture sur une surface maximale d'un hectare qu'ils louent en fermage ou en métayage, ou qu'ils occupent illégalement dans la Réserve.

Type 2. Les «petits-moyens caféiculteurs aux cultures diversifiées» combinent la production de café avec la production de cultures vivrières, parfois complétées par un élevage bovin, sur une exploitation de 1 à 7 ha.

Type 3. Les producteurs de cultures vivrières avec des systèmes agroforestiers caféiers ont une stratégie centrée sur la production de cultures vivrières combinée à une petite production de café. Avec des surfaces comprises entre 3 et 8 ha, ils emploient des salariés pendant la récolte. La majorité de ces producteurs ont des bovins qui fournissent du lait pour l'autoconsommation et peuvent servir d'assurance en cas de sinistre.

\section{Les exploitations patronales}

Type 4. Les grands caféiculteurs spécialisés ont des exploitations de 29 à 36 ha dédiées à la production de café en système agroforestier, avec des salariés agricoles. Ils n'ont pas de cultures vivrières, mais certains élèvent des bovins.

Type 5. Les grands caféiculteurs diversifiés possèdent des exploitations de 17 à 480 ha cultivés avec des salariés. Ils combinent une parcelle de caféiers de grande taille (jusqu'à 77 ha) avec une production annuelle relativement élevée, des cultures vivrières et l'élevage bovin.

\section{Les haciendas}

Type 6. Les «haciendas caféières» d'une surface de 161 à 479 ha détiennent de grandes parcelles de caféiers en systèmes agroforestiers (140 à 150 ha) avec une 
production annuelle importante. Les propriétaires de ces haciendas engagent des salariés et produisent plus de 25 tonnes/an de café parche. Ces haciendas ne produisent pas de cultures vivrières. Elles se distinguent des exploitations patronales par leur taille et le nombre de salariés (70 permanents et 500 saisonniers). Le niveau d'éducation du propriétaire est élevé, comprenant un cursus universitaire à l'étranger avant son installation au Nicaragua. Il dispose d'un réseau professionnel et d'un capital personnel importants.

\section{Exploitations agricoles et systèmes agroforestiers caféicoles au Costa Rica}

Le site de Llano Bonito (Costa Rica) est dominé par la culture du café. La région est relativement homogène du point de vue des systèmes de culture. Le caféier est cultivé en systèmes agroforestiers simples avec peu de diversité arborée. Depuis les limitations au changement d'usage des sols (loi forestière nº7575 de 1996), les parcelles de forêt rémanente ne peuvent pas être converties à l'agriculture. Sur les terres les plus hautes (1900-2000 m dans la vallée de Llano Bonito), les pâturages existants sont progressivement plantés en caféiers en raison de l'augmentation des températures. Les deux espèces pourvoyeuses d'ombrage complantées dans les caféières sont Erythrina poeppigiana (et quelques autres espèces de même genre telles que E. fusca, E. glauca et E. berteroana) - légumineuse fixatrice d'azote - et Musa sp. pour la production de bananes. Quelques arbres fruitiers, dont l'avocatier (Persea americana), des espèces d'agrumes et quelques rares espèces d'arbres à bois d'œuvre sont parfois aussi présents. Les itinéraires techniques des systèmes agroforestiers caféicoles sont relativement peu différenciés. Les principales différenciations concernent la gestion de l'ombrage, la fertilisation et la lutte contre les adventices (Bonifazi, 2015; Dhorne, 2013; Laffourcade, 2012). Deux groupes d'agriculteurs se détachent assez nettement : les agriculteurs patrimoniaux et les exploitants expérimentateurs.

\section{Les agriculteurs patrimoniaux : type 1}

Les agriculteurs patrimoniaux sont de petits producteurs ayant une surface de 3,1 ha en moyenne. Ils ont appris très jeunes à cultiver les caféiers avec leur père : «Je me contente de faire comme mon père, le même système que lui ». Ils ne modifient que rarement leurs pratiques et se fient à leur grande expérience et à celle de leurs parents ou aïeux. Ils s'intéressent aux cours ou formations dont ils appliquent rarement les recommandations : «C'est très dur d'apprendre». Toutefois, il leur arrive de changer leurs modes de production lorsqu'un conseil est relayé par plusieurs organismes sur une longue durée, ou lorsqu'ils constatent qu'une pratique nuit à leur production, leur terre ou leur santé.

\section{Les agriculteurs expérimentateurs : types 2, 3, 4}

Les agriculteurs de ce groupe sont des producteurs qui testent des pratiques nouvelles : changer les espèces d'arbres d'ombrage, varier la quantité, la fréquence 
d'utilisation et la marque des intrants. Ils ont souvent fait des études longues (au-delà du baccalauréat) ou ont appris à cultiver le caféier auprès de différents tuteurs. Ce groupe rassemble trois types de producteurs.

Type 2. Les grands producteurs. Ils ont une exploitation de 25,2 ha en moyenne, ce qui leur donne une marge de manœuvre plus grande pour expérimenter de nouvelles pratiques. En moyenne, seule la moitié de la surface de leur exploitation est dédiée au système agroforestier à base de caféier. Sur le reste, ils élèvent du bétail, conduisent d'autres cultures (avocatiers) ou conservent des forêts.

Type 3. Les fournisseurs de Volcafé. Ces producteurs sont peu nombreux dans la zone. La surface de leur exploitation est en moyenne de 19,3 ha. Ils sont en contact avec les certificateurs, ces derniers s'assurant que la majoration de prix soit justement attribuée : «Ils (les certificateurs de Volcafé) font une inspection, contrôlent tout ... mais cela me plaît, c'est très sain pour l'environnement». Cette rémunération des pratiques vertes est une motivation pour modifier les comportements dans le sens de la protection de l'environnement.

Type 4. Ce sont des expérimentateurs ayant une surface assez faible et qui aiment le changement. Malgré la faible marge de manœuvre dont ils disposent (leur surface moyenne est de 2,4 ha), ils essaient souvent de nouvelles cultures ou l'élevage. Ces petits expérimentateurs ont des exploitations de quatre hectares au plus et ont pour la plupart appris à cultiver le caféier pendant leurs études ou auprès de leur entourage (au-delà de l'entourage familial) : «Je suis technicien agricole, je n'exerce pas la fonction mais le peu que j'ai appris, je le vis sur ma ferme», «J'ai appris toute ma vie des voisins, des amis et par mes propres efforts ».

\section{Pressions environnementalistes et stratégies des agriculteurs, influence de la situation économique et des institutions}

$\mathrm{Au}$ Nicaragua, bien que conscients des défis environnementaux, en particulier lorsqu'ils affectent des questions de santé publique (pollution de l'eau), les agriculteurs les plus pauvres, souvent peu dotés en foncier, n'ont pas d'autre choix que de prioriser leur sécurité alimentaire et l'obtention de revenus. Cette stratégie est adoptée même si elle implique de couper des arbres, d'utiliser des intrants agrochimiques ou de rejeter des effluents dans la nature.

Au Costa Rica, le revenu moyen, plus élevé des exploitations en comparaison de celles du Nicaragua, facilite des choix plus en adéquation avec le discours environnementaliste ambiant.

\section{À La Dalia, Nicaragua}

Les acteurs perçoivent deux principaux enjeux environnementaux (Baranger et al., 2015 ; Dorgans-Cadihac et al., 2013) :

- la pollution de l'eau attribuée à l'utilisation de produits agrochimiques dans l'agriculture et aux effluents de la première transformation du café;

- la destruction du couvert forestier et la diminution du nombre d'arbres hors-forêt.

Toutefois, tous les acteurs ne peuvent pas adopter des stratégies en accord avec les impératifs de préservation de l'environnement. 
Les producteurs familiaux ayant des surfaces limitées ont une stratégie centrée sur l'autosuffisance alimentaire, avec une petite production de café lorsque leur dotation foncière le permet. Leur objectif est d'acquérir le foncier et d'augmenter leur production de café, parfois en déboisant, afin de capitaliser dans l'élevage bovin et les caféières. Cependant, ces agriculteurs sont souvent dans un cercle «vicieux». En effet, l'essentiel de leurs ressources est utilisé dans le cycle de production suivant et ils ne réussissent pas à capitaliser et améliorer leur niveau de vie.

Les salariés agricoles ayant une petite parcelle en cultures vivrières ont un faible niveau d'éducation et ne se projettent pas dans l'avenir. Cela constitue un obstacle quant à l'amélioration de leurs pratiques agricoles et à leur sensibilisation aux questions environnementales.

Les exploitants familiaux membres de coopératives (parmie les types 2 et 3 ) et les exploitants patronaux (types 4 et 5) sont en relation avec de nombreux acteurs. Cette proximité leur permet de bénéficier des programmes d'appui et des actions incitatives mis en œuvre par les institutions. En revanche, les exploitants familiaux non-membres de coopératives (parmi les types 2 et 3 , et ceux de type 1), les petits et moyens caféiculteurs ayant des cultures diversifiées et les propriétaires des haciendas (type 6) sont faiblement touchés par ces institutions.

La majorité des grands caféiculteurs diversifiés (type 5) ont fait certifier leur production et valorisent la qualité de leur café au meilleur prix. Les incitations ne sont pas efficaces pour impulser un changement de pratiques aux propriétaires des haciendas (type 6). Ceux-ci privilégient la maximisation de leurs profits (notamment par rapport à leur capital foncier) en cultivant du caféier en monoculture de plein soleil et en utilisant beaucoup d'intrants agrochimiques. Ils ne peuvent donc pas remplir le cahier des charges des certifications.

Pour tous ces agriculteurs, les programmes d'éducation et de sensibilisation ne comprennent pas de suivi technique. La plupart des actions incitatives manquent aussi de coordination. Il en résulte une limitation de la diffusion du discours environnementaliste et de l'apprentissage de pratiques «vertes».

Le niveau d'application des réglementations est faible ou partiel, qu'elles soient incitatives ou coercitives. En conséquence, le déboisement n'est pas limité. Les exploitations familiales, les plus nombreuses, sont les plus affectées par certaines amendes, notamment celles liées à l'abattage d'arbres. Les entreprises patronales et les haciendas sont davantage exposées aux amendes liées à une mauvaise gestion de leurs effluents issus de la première transformation du café.

\section{À Llano Bonito, Costa Rica}

Les discours des organisations et des institutions se retrouvent dans celui des agriculteurs. Lérosion des sols et la dangerosité chimique des herbicides pour l'environnement et pour la santé sont souvent évoquées. Les avis sont plus partagés en ce qui concerne les autres produits phytosanitaires. Les problèmes de pollution liés aux fertilisants azotés sont passés sous silence tant par les institutions que par les agriculteurs, malgré un usage conduisant à une pollution dépassant la norme préconisée (teneur en nitrate inférieure à 40 mg/l dans les eaux de drainage) (Cannavo et al., 2013). 
Dans les exploitations de grands producteurs, en moyenne, seulement la moitié de la surface est couverte de caféiers. Les grands producteurs possèdent des pâturages ou de la forêt sur leurs terres. Contrairement aux autres agriculteurs, ils ont les ressources financières et foncières ou sont contraints par la loi de conserver ainsi des terres non productives. Ils justifient la conservation de forêt par conviction personnelle.

Les petits propriétaires, petits expérimentateurs ou exploitants patrimoniaux, possèdent presque exclusivement des parcelles de caféiers et sont seuls à travailler sur leurs exploitations. Cette situation leur laisse, d'une part, une faible marge de manœuvre financière et foncière et, d'autre part, une plus grande marge de manœuvre technique par rapport aux grands producteurs. En effet, ils peuvent adapter leurs pratiques à chacune de leurs parcelles et aux conditions externes (climat, maladies, ...). Par exemple, les petits expérimentateurs utilisent un minimum d'herbicides à raison de 0,5 passage/an - autrement dit, un passage tous les deux ans - contre 0,92 passage/an pour les grands producteurs. Ils le font avec parcimonie : «Je mets de l'herbicide seulement sur les mauvaises herbes qui ne partent pas autrement».

Outre les dangers des herbicides, les petits expérimentateurs soulignent les problèmes d'érosion dans la région. Ils ont en général une couverture arborée qui comporte des arbres fruitiers. Produire des légumes sous serre, planter des arbres fruitiers dans la caféière ou commencer des activités d'éco-tourisme sont des projets envisagés.

Les agriculteurs patrimoniaux ont en général un profil plus productiviste. La moitié d'entre eux dénonce l'usage des herbicides ou s'inquiète de l'érosion. Cependant, ils utilisent beaucoup de fertilisants et sont ceux qui épandent le plus souvent d'herbicides (0,92 passage/an). Ils diversifient peu leur couverture arborée. Le contexte foncier dans la zone empêche bien souvent ces agriculteurs d'acheter des terres; ils adoptent alors la voie de l'intensification à l'hectare.

En général, les agriculteurs répondent de manière cohérente aux problèmes environnementaux qu'ils perçoivent. Ils mettent en place des pratiques agricoles qui réduisent l'usage des intrants chimiques, sauf les deux fournisseurs de Volcafé qui emploient plus de fertilisants chimiques et de fongicides que la moyenne des agriculteurs de la zone. Ce hiatus est un reflet de la certification Nespresso AAA obtenue par Volcafé : la limitation des herbicides et la diversification de la couverture arborée sont des exigences, alors que les limitations en fertilisants chimiques et fongicides ne sont que des recommandations.

Tous les agriculteurs fustigent les herbicides et n'en utilisent quasiment pas. Les grands propriétaires soulignent l'importance de conserver des forêts et en maintiennent sur leurs terrains; ils ne se soucient guère des produits phytosanitaires qu'ils ont tendance à utiliser davantage que les plus petits producteurs. Inversement, les petits agriculteurs ont peu de forêts et utilisent peu de produits phytosanitaires. Les grands propriétaires ne manipulent pas les produits phytosanitaires, déléguant cette tâche à leurs employés. Les petits agriculteurs le font eux-mêmes. Cette disparité de pratique explique largement la différence de sensibilité à la dangerosité des produits. 


\section{Conclusion}

À La Dalia au Nicaragua, les agriculteurs gèrent des systèmes agroforestiers variés et riches en agrobiodiversité : nombreuses variétés de fruitiers et d'arbres forestiers utilisés dans l'ombrage des caféiers. Llano Bonito au Costa Rica est dominé par des caféiers conduits en quasi-monoculture sous ombrage d'une à trois espèces arborées.

$\mathrm{Au}$ Nicaragua, de grandes différenciations socio-économiques (dotation foncière, infrastructures de transformation et, in fine, de revenus) existent entre les agriculteurs. La plupart des agriculteurs pratiquent une agriculture vivrière, une minorité possède de grands domaines. Le territoire comprend plus d'agrobiodiversité, mais celle-ci est menacée par le fait que les populations aspirent légitimement à une amélioration de leur niveau de vie - en privilégiant d'abord l'augmentation de la production par l'usage de produits agrochimiques - à la mise en œuvre de pratiques plus respectueuses de l'environnement. La différenciation sociale est forte entre les agriculteurs avec notamment des cas de pauvreté, ce qui limite l'appropriation des discours environnementalistes.

Au Costa Rica, l'évolution du conseil et de l'encadrement agricole de la caféiculture au cours du $\mathrm{Xx}^{\mathrm{e}}$ siècle a entraîné une standardisation des pratiques consommatrices de produits agrochimiques et génératrices d'érosion, dans un modèle d'agriculture tourné vers l'exportation. Cela s'est traduit par un appauvrissement de la biodiversité.

Les pressions environnementalistes sont assez similaires dans les deux pays, mais les résultats sont différents.

Au Costa Rica, l'homogénéité et la force des discours ont produit une idéologie environnementaliste globale qui s'applique à une situation relativement homogène en termes de systèmes de production et de niveau socio-économique. Néanmoins, un décalage existe encore entre le discours des agriculteurs et leurs pratiques. Au Nicaragua, les multiples pressions environnementalistes se heurtent à une grande diversité de situations en termes de systèmes de production et de niveau socio-économique, ainsi qu'aux priorités relevant de la survie des populations les plus précaires.

\section{Remerciements}

Nous remercions vivement les populations locales qui nous ont toujours aimablement accueillis. Merci à Louise Begey et Casi Avard pour l'aide à l'édition de ce chapitre.

\section{Bibliographie}

Arribard L., 2013. Production agricole et préservation des ressources naturelles au Nicaragua : analyse diagnostic du système agraire à l'ouest du massif de Peñas Blancas. Mémoire de Master. Nanterre, AgroParisTech, Université Paris Ouest.

Baranger M., Fréguin-Gresh S., Le Coq J.-F., Rapidel B., Pesche D., Aubert P.-M., 2015. Services écosystémiques, stratégies d'acteurs et relations sociales dans un territoire agroforestier au Nicaragua : le cas de la commune de La Dalia. Montpellier : Cirad, 83. 
Bonifazi M., 2015. Services écosystémiques et production agroforestière : instruments d'incitation pour le bassin de Llano Bonito, Costa Rica. Mémoire de Master. Montpellier : SupAgro, 100.

Cannavo P., Harmand J.-M., Zeller B., Vaast P., Ramirez J.-E., Dambrine E., 2013. Low nitrogen use efficiency and high nitrate leaching in a highly fertilized Coffea arabica-Inga densiflora agroforestry system: a 15N labeled fertilizer study. Nutrient cycling in agroecosystems, 95(3): 377-394.

Cenagro, 2012. Informe final Iv Censo nacional agropecuario. Cenagro. www.inide.gob.ni/Cenagro/ infivcenagro/informefinal.html.

Constanty M., 2015. Stratégie des acteurs dans la gestion des déchets de l'usinage du café au Costa Rica : un exemple d'intégration de contraintes environnementales par les acteurs d'une filière agricole. Mémoire de fin d'études : Gestion environnementale des écosystèmes et forêts tropicales. Montpellier : SupAgro, Montpellier, 68 p.

Dhorne S., 2013. Environnement et stratégies des acteurs dans les systèmes agroforestiers caféicoles : le cas du district de Llano Bonito (Costa Rica), Mémoire, Environnement, développement, territoires et sociétés. Mémoire de Master. Paris : Muséum national d'histoire naturelle, AgroParisTech, $61 \mathrm{p}$.

Dorgans-Cadihac J., Freguin-Gresh S., Pedelahore P., Marsden C., Sibelet N., 2013. Environmentalist pressure, stakeholder perception and productive strategy in an agricultural Region bordering a Nature Reserve (La Dalia, Nicaragua), In : Henry A. Wallace, Inter-American Scientific Conference. Turrialba, Costa Rica, 2013-09-30 / 2013-10-04, s.n. Communication par affiche, 1 p.

Dorgans-Cadilhac J., 2013. Stratégies et jeux d'acteurs, entre développement de l'agriculture et conservation des ressources naturelles de part et d'autre d'une aire protégée. Analyse d'une étude de cas dans la commune d'El Tuma-La Dalia, Nicaragua. Mémoire de Master. Montpellier, France, Institut des régions chaudes-SupAgro.

Fréguin-Gresh S., Wilson White C., Flores López J.C., Müller Oporta P.E., Huybrechs F., Pikitle A., Marchena Williams R., Manzanares D., 2014. Mapping institutions that govern access and uses of natural resources in the Nicaragua-Honduras sentinel landscape: Revealing the complexity, issues, and challenges of natural resource governance. Montpellier: Cirad, $126 \mathrm{p}$.

Gómez L., Munk Ravnborg H., Castillo E., 2011. Gobernanza en el uso y acceso a los recursos naturales en la dinámica territorial del Macizo de Peñas Blancas: Nicaragua. Santiago de Chile: Rimisp, Centro latinoamericano para el desarrollo rural, Documento de trabajo ${ }^{\circ} 82$. https://idl-bnc-idrc. dspacedirect.org/bitstream/handle/10625/46792/133246.pdf? sequence=1 (consulté le 07/04/2019).

Kaufmann J.-C., 2011. Lentretien compréhensif. Paris : Armand Colin, 128 p.

Laffourcade R., 2012. Discours environnementaliste : de la théorie aux pratiques agricoles, entre retouches et renouveaux, Mémoire de Master. Paris : AgroParisTech, 67.

Melendez Marin L., 2010. Plan de manejo de cuenca del Rio Pirris, Instituto costarricense de electricidad, UEN PySA, Proyecto Hidroelectrico Pirris, San José, Costa Rica.

Meylan L., Merot A., Gary C., Rapidel B., 2013. Combining a typology and a conceptual model of cropping system to explore the diversity of relationships between ecosystem services: the case of erosion control in coffee-based agroforestry systems in Costa Rica. Agricultural systems, 118: 52-64.

Meylan L., Gary C., Allinne C., Ortiz J., Jackson L., Rapidel B., 2017. Evaluating the effect of shade trees on provision of ecosystem services in intensively managed coffee plantations. Agriculture, ecosystems and environment, 245: 32-42.

Rosero-Bixby L., Dow W.H., 2012. Predicting mortality with biomarkers: a population-based prospective cohort study for elderly Costa Ricans. Population health metrics, 10(1): 1.

Sibelet N., 2015. Reconnaissance des capacités d'innovation des agriculteurs face aux pressions environnementalistes : projet scientifique. Cirad, Turrialba, Costa Rica, 23 p.

Sibelet N., Mutel M., Arragon P., Luye M., Pollet S., 2013. Méthodes de l'enquête qualitative appliquée à la gestion des ressources naturelles. Course en ligne. Montpellier, France, Cirad, IAMM, SupAgro, Uved. http://entretiens.iamm.fr/.

Snider A., Kraus E., Sibelet N., Bosselmann A., Faure G., 2016. Influence of voluntary coffee certifications on cooperatives' advisory services and agricultural practices of smallholder farmers in Costa Rica. Journal of agricultural education and extension, 22(5): 435-453. 



\title{
Chapitre 2
}

\section{Suivi des services écosystémiques dans un observatoire de caféiers agroforestiers. Applications pour la filière du café}

\author{
Roupsard O., Allinne C., Van den Meersche K., Vaast P., Rapidel B., \\ Avelino J., Jourdan C., Le Maire G., Bonnefond J.-M., Harmand J.- \\ M., Dauzat J., Albrecht A., Chevallier T., Barthès B., Clément- \\ Vidal A., Gomez-Delgado F., Charbonnier F., Benegas L., Welsh K., \\ Kinoshita R., Vézy R., Pérez-Molina J.-P., Kim J., Taugourdeau S., \\ Defrenet E., Nespoulous J., Rançon F., Guidat F., Cambou A., Soma M., \\ Mages C., Schnabel F., Prieto I., Picart D., Duthoit M., Rocheteau \\ A., Do F., de Melo Virginio Filho E., Moussa R., Le Bissonnais Y., \\ Valentin C., Sanchez-Murillo R., Roumet C., Stokes A., Vierling L., \\ Eitel J., Dreyer E., Saint-André L., Malmer A., Loustau D., IsAac M., \\ Martin A., Priemé A., Eberling B., Madsen M., Robelo A., Robelo D., \\ Borgonovo C., Lehner P., Ramirez G., Jara M., Acuña Vargas R., \\ Barquero A., Fonseca C. et Gay F.
}

Résumé. Huit ans de travaux de recherche sur les services écosystémiques dans une grande ferme caféière du Costa Rica (observatoire collaboratif Coffee-Flux, en système agroforestier à base de caféiers sous de grands arbres d'Erythrina poeppigiana, surface projetée de couronne de l'ordre de 16\%) ont suggéré plusieurs applications pour les agriculteurs et les décideurs. Il est apparu que de nombreux services écosystémiques dépendaient des propriétés du sol (ici des Andisols), en particulier de l'érosion, de l'infiltration, de la capacité de stockage de l'eau et des éléments nutritifs. Nous confirmons qu'il est essentiel de lier les services hydrologiques et de conservation au type de sol en présence. Une densité adéquate d'arbres d'ombrage (plutôt faible ici) permet de réduire la sévérité des maladies foliaires avec, en perspective, une réduction de l'usage de pesticides-fongicides. Un simple inventaire de la surface basale au collet des caféiers permet d'estimer la biomasse souterraine et la moyenne d'âge d'une plantation de caféiers, ce qui permet d'évaluer sa 
valeur marchande ou de planifier son remplacement. Le protocole de calcul actuel pour la neutralité carbone des systèmes agroforestiers ne prend en compte que les arbres d'ombrage, pas la culture intercalaire. Dans la réalité, si on inclut les caféiers, on se rapproche très probablement de la neutralité. Des évaluations plus complètes, incluant les arbres, les caféiers, la litière, le sol et les racines dans le bilan en carbone du système agroforestier sont proposées. Les arbres d'ombrage offrent de nombreux servies écosystémiques s'ils sont gérés de manière adéquate dans le contexte local. Par rapport aux parcelles en plein soleil, nous montrons qu'ils réduisent l'érosion laminaire d'un facteur 2, augmentent la fixation de l'azote $\left(\mathrm{N}_{2}\right)$ atmosphérique et le pourcentage d'azote recyclé dans le système, réduisant ainsi les besoins en engrais. Ils réduisent aussi la sévérité des maladies foliaires, augmentent la séquestration de carbone, améliorent le microclimat et atténuent substantiellement les effets des changements climatiques. Dans notre étude de cas, aucun effet négatif sur le rendement n'a été enregistré.

\begin{abstract}
Eight years of studying coffee ecophysiology and monitoring ecosystem services (ES) in a large coffee farm in Costa Rica revealed several practical recommendations for farmers and policy makers. The cropping system studied within our collaborative observatory (Coffee-Flux) corresponds to a coffee-based agroforestry system (AFS) under the shade of large trees of Erythrina poeppigiana (16\% of canopy cover). A lot of ES and disservices depend on local soil properties (here Andisols), especially erosion/infiltration, water/carbon and nutrient storage capacity. Therefore, for ES assessment, the type of soil is crucial. An adequate density of shade trees (rather low here) reduced the severity of leaf diseases with the prospect of reducing pesticide-fungicide use. A simple inventory of the basal area at collar of the coffee plants allowed estimating the belowground biomass and the average age of the plantation, to judge of its market value and to decide when to replace it. Coffee farms are probably much closer to $\mathrm{C}$ neutrality than predicted by the current C-Neutral protocol, which only considers shade trees. More comprehensive assessments, including trees, coffee, litter, soil, and roots in the $\mathrm{C}$ balance of the AFS are proposed. Shade trees offer many ES if they are adequately managed in the local context. As compared to full sun conditions, shade trees may (i) reduce laminar erosion by a factor of 2, (ii) increase $\mathrm{N} 2$ fixation and the $\%$ of $\mathrm{N}$ recycled into the system, thus reducing fertilizer requirements, (iii) reduce the severity of leaf diseases, (iv) increase C sequestration, (v) improve the microclimate, and (vi) substantially reduce the effects of climate change. In our case study, no negative effect on coffee yield was found.
\end{abstract}

\title{
Introduction
}

Le caféier est une culture pérenne tropicale cultivée dans environ 80 pays. La consommation mondiale de café est supérieure à 9 millions de tonnes, dont environ $65 \%$ proviennent de Coffea arabica L. On estime que l'ensemble de la chaîne de valeur du café (de la culture au marketing) implique environ 100 millions de personnes dans le monde. Aujourd'hui, les certifications Nama-Café ${ }^{1}$ et C-Neutre 2 promeuvent les modes de culture permettant l'atténuation et l'adaptation aux changements climatiques. Beaucoup de recherches fructueuses sont publiées chaque année sur le café, mais il n'existait pas d'observatoire collaboratif sur un même site avec des approches multidisciplinaires et verticales (même unité de lieu et de

1. https://www.namacafe.org/

2. Certification C-neutre Costa Rica : https://presidencia.go.cr/comunicados/2016/09/meta-de-carbononeutralidad-para-el-2021-se-mantiene-y-fortalece/. 
temps). Un tel observatoire offre la possibilité de relier plusieurs disciplines de recherche et d'avoir une vue d'ensemble et à long terme de l'écophysiologie du caféier dans des conditions réelles.

En 2009, le Cirad, le Catie et la ferme d'Aquiares ${ }^{3}$ ont créé leur observatoire collaboratif à long terme «Coffee-Flux » ${ }^{4}$ pour l'étude biophysique du système agroforestier caféier (fig. 2.1). La ferme d'Aquiares est située dans le bassin du Reventazón, dans la région Centre-Caraïbes du Costa Rica, aux coordonnées 9 ${ }^{\circ} 56^{\prime} 17^{\prime \prime}$ (Nord) et 8343'41" (Ouest), sur la pente du volcan Turrialba. Certifiée Carbon-Neutral et Rainforest Alliance ${ }^{\mathrm{TM} 5}$, c'est l'une des plus grandes fermes du Costa Rica $\left(6,6 \mathrm{~km}^{2}\right)$. La parcelle principale où sont installés les principaux instruments de mesure (tourà-flux, venturi) est à $1000 \mathrm{~m}$ d'altitude, dans un bassin hydrographique de $1 \mathrm{~km}^{2}$.

Lobjectif de Coffee-Flux est d'évaluer les flux de carbone, d'eau, de nutriments, de $\mathrm{N}_{2} \mathrm{O}$ et d'érosion et de quantifier les services écosystémiques, de la plante jusqu'au bassin versant ou à la ferme. Lobservation, l'expérimentation, la modélisation et la télédétection sont combinées. Les données sont collectées (fig. 2.2) et les modèles sont calibrés localement, de la plante à la parcelle (ha) avant de changer d'échelle (bassin, ferme). Lobservatoire est suivi depuis 2009 afin d'appréhender les fluctuations saisonnières et interannuelles de la productivité du caféier et les services écosystémiques associés. Lobservatoire Coffee-Flux a été soutenu en permanence par plusieurs institutions et projets, dont le projet Safse (Compromis entre production et autres services écosytémiques fournis par les systèmes agroforestiers, Cirad/IRD).

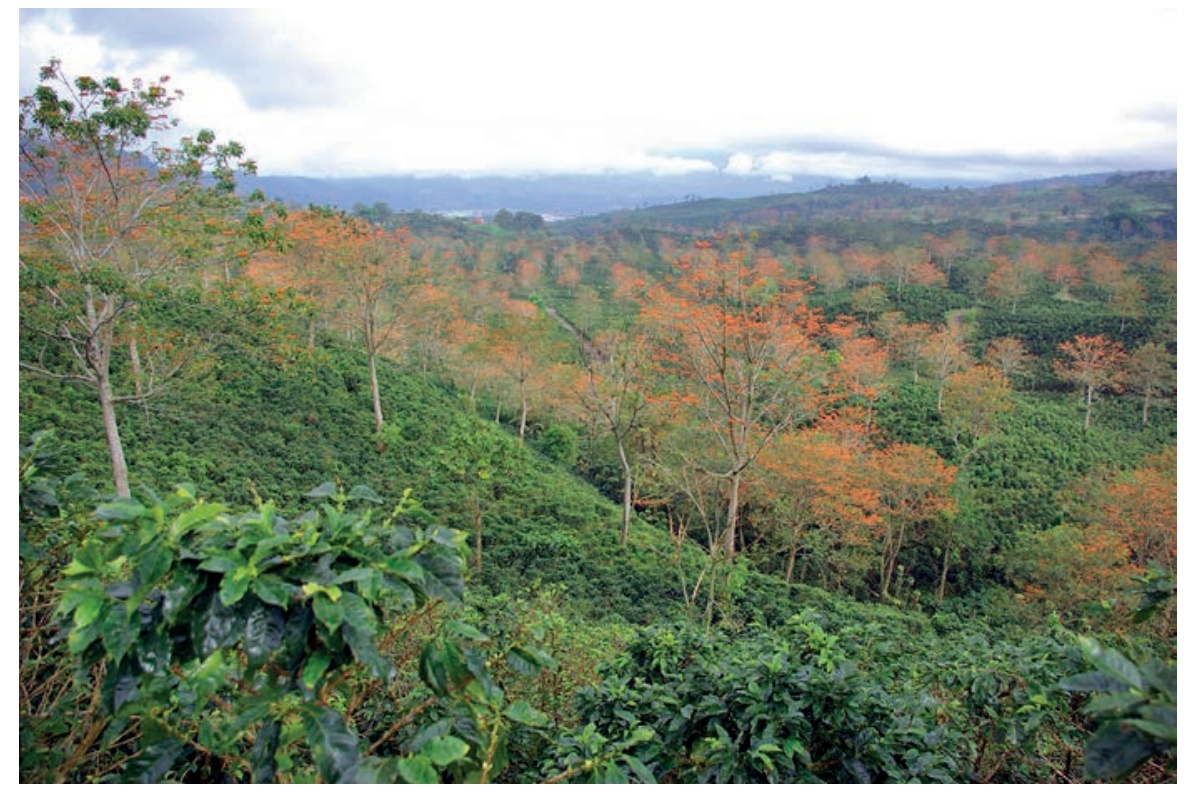

Figure 2.1. Lobservatoire collaboratif CoffeeFlux dans la ferme d'Aquiares : caféiers sous grandes érythrines.

3. Cafetalera Aquiares : http://auction.stoneworks.com/includes/crq2004/aquiares_brochure.html

4. Coffee-Flux Observatory: http://www.umr-ecosols.fr/index.php/fr/recherche/projets/53-coffee-flux.

5. Rainforest-Alliance ${ }^{\mathrm{TM}}$ : https://www.rainforest-alliance.org/lang/fr. 


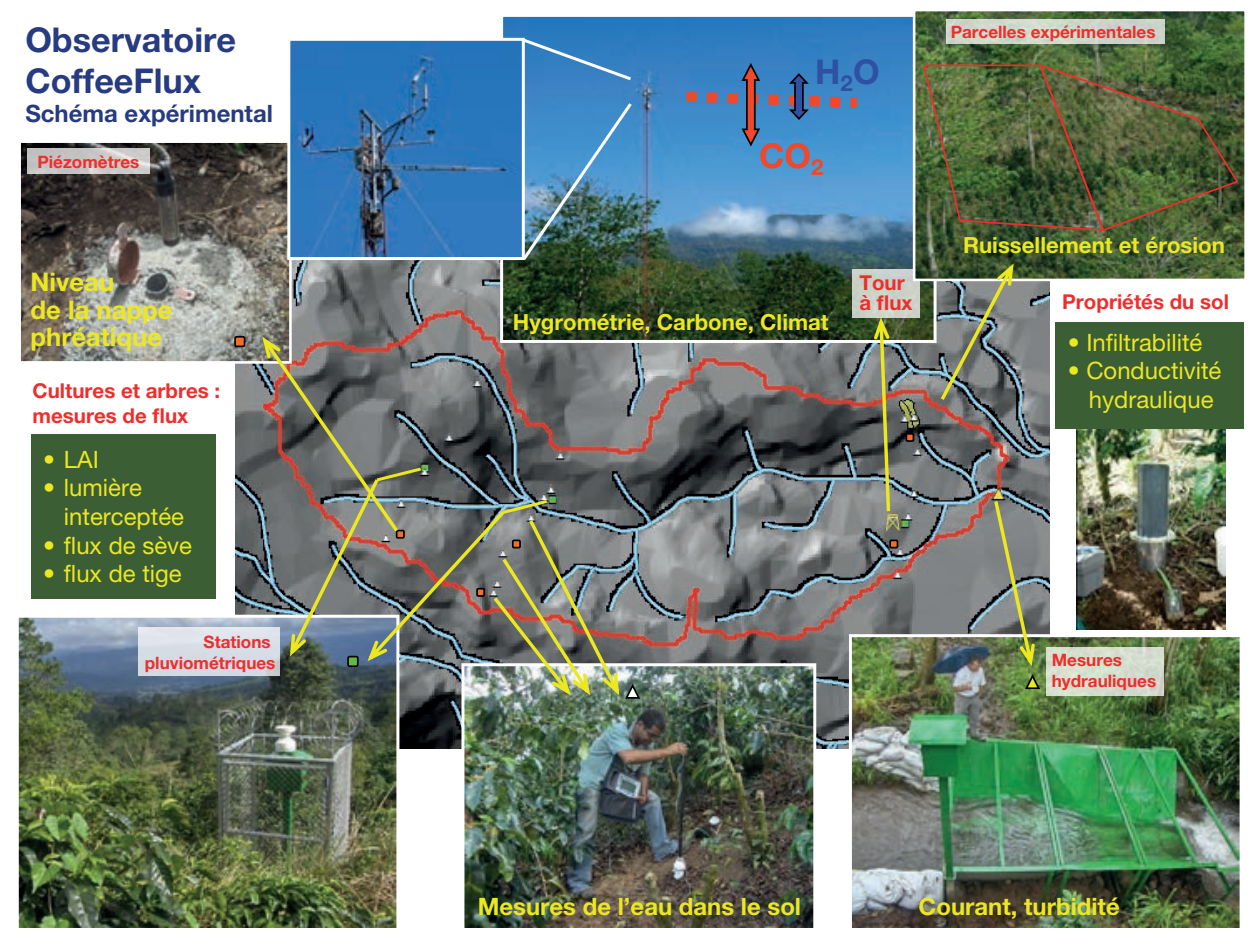

Figure 2.2. Dispositif expérimental de l'observatoire collaboratif CoffeeFlux dans la ferme d'Aquiares : mesures et modélisations des flux de $\mathrm{C}, \mathrm{H}_{2} \mathrm{O}$, nutriments, de l'érosion, des services écosystémiques dans un système agroforestier à base de caféier.

La philosophie est de concentrer plusieurs recherches sur un site spécifique et pendant plusieurs années, de partager une base de données expérimentale commune, de développer la modélisation et de publier des résultats dans des revues scientifiques de haut rang. Cette recherche collaborative a été possible grâce à des interactions positives entre plusieurs institutions (Isaac et al., 2017; Kim et al., 2017; Martin et al., 2017; Prieto et al., 2015; Sánchez-Murillo et al., 2016). La recherche appliquée a été également fortement encouragée (Roupsard et al., 2017) : en agronomie, sur la sélection de variétés, pour les certifications C-Neutre et NAMA (De Melo Virginio Filho et al., 2015; Roupsard, 2015). Coffee-Flux bénéficie de l'infrastructure du Catie, d'un accès facile au terrain et d'une bonne sécurité. La base de données (disponible sous $\mathrm{R}$ ) accélère le partage.

Notre objectif ici est de présenter les principales réalisations de Coffee-Flux après huit ans de suivi, soulignant l'intérêt pratique pour les producteurs de café et les décideurs. Nous nous concentrerons sur : les stocks de carbone des sols; l'hydrologie; l'érosion, la fertilisation, l'ombrage et le rendement; la fertilisation et les flux de $\mathrm{N}_{2} \mathrm{O}$; la sévérité du complexe des maladies foliaires et l'ombrage; l'indice foliaire et le rendement du caféier; les racines; les effets de l'ombrage sur la productivité primaire du caféier et sur son efficience d'utilisation de la lumière; le bilan de carbone de l'écosystème; les arbres d'ombrage et leur adaptation aux changements climatiques. 


\section{W Quelques applications pratiques de Coffee-Flux}

\section{Stock de carbone très élevé et grande variabilité horizontale dans les andisols}

La plupart des sols sous caféiers dans la région étudiée sont des andisols, ou leurs versions plus évoluées (ultisols). Les andisols proviennent de cendres volcaniques régulièrement déposées, ici par le volcan Turrialba. Ces cendres évoluent en allophanes. Puis avec le temps en argiles, les andisols deviennent alors des ultisols.

Les andisols sont des sols jeunes et riches en allophanes (jusqu'à 20\%). Plus il y a d'allophanes, plus fortes sont leurs propriétés andiques. Les allophanes ont une structure nanométrique avec une très grande surface. Ils peuvent piéger beaucoup d'eau, de carbone et d'éléments minéraux tels que le phosphore. Les contaminants sont probablement accumulés dans ces sols lors de leur formation. Le pourcentage d'allophanes est très corrélé avec les stocks de carbone des sols. Les stocks de carbone sont élevés, de l'ordre de $560 \pm 180$ t C/ha ou $56 \pm 18 \mathrm{~kg} \mathrm{C} / \mathrm{m}^{2}$ entre 0 et $2 \mathrm{~m}$ (Chevallier et al., résultats préliminaires), soit un ordre de grandeur dix fois plus élevé que la biomasse aérienne et souterraine évaluée à 37 t C/ha (Charbonnier et al., 2017; Defrenet et al., 2016).

Avec le temps, les allophanes sont lessivés et transformés en une séquence d'argiles : la teneur en carbone peut être extrêmement variable à l'échelle d'une ferme (Kinoshita et al., 2016), comme révélé par spectométrie infrarouge. Le pourcentage d'allophanes (ou le rapport allophanes/argiles) étant le principal déterminant de la variabilité spatiale des stocks de carbone des sols, il faut l'appréhender avant d'évaluer les stocks de carbone des sols dans les fermes ou entre fermes.

\section{Applications pratiques}

En fonction du rapport allophanes/argiles, les besoins en fertilisation pourraient être très différents, même au sein de petites zones apparemment homogènes. La mise à disposition de cartes détaillées des andisols agricoles permettrait aux agriculteurs d'économiser des engrais et de minimiser leur impact sur le sol et l'eau. La spectrométrie infrarouge est un outil prometteur pour le criblage des sols à haut débit.

\section{Grande infiltrabilité des andisols, le bilan hydrologique piloté par l'aquifère}

Les parcelles avec andisols d'Aquiares infiltrent environ $92 \%$ de la pluie (GómezDelgado et al., 2011). Le ruissellement de surface au sein des parcelles est donc très faible, sa contribution au débit de rivière n'est que de $4 \%$. Les andisols sont une «éponge » avec une teneur élevée en eau permise par les allophanes, une macroporosité élevée, un fort drainage profond et une infiltration très élevée ( $>1000 \mathrm{~mm} / \mathrm{heure}$ ) (Benegas et al., 2014; Hasselquist et al., 2018). Le débit de la rivière dépend principalement des aquifères (56\%) (Gómez-Delgado et al., 2011), notamment via des sources (Welsh et al., 2018). 


\section{Applications pratiques}

La gestion sans labour du caféier semble bénéficier à l'aquifère dans les andisols. Cependant, en raison d'une infiltration importante, les contaminants transitent probablement dans l'aquifère. Il est recommandé de surveiller le niveau et la qualité des aquifères dans de tels sols, notamment dans les régions plus sèches où des effets de concentration sont probables.

\section{Effet prépondérant du type de sol par rapport à celui de la présence d'arbres d'ombrage sur l'érosion}

Les andisols présentent une faible érosion laminaire, en cohérence avec les faibles ruissellements de surface mesurés. La production de sédiments par le bassin versant est très faible (environ $1 \mathrm{t} / \mathrm{ha} / \mathrm{an}$ ). Très peu de sédiments (environ $5 \%$ ) proviennent des parcelles caféières elles-mêmes. Les deux tiers des sédiments proviennent en fait du lit de la rivière. Le reste (environ 1/3) provient des routes et des sentiers situés entre les parcelles caféières. Le ruissellement est en effet élevé sur les routes provoquant une érosion des bordures de parcelles. Par conséquent, l'érosion ne doit pas être considérée à l'échelle de la parcelle, mais à l'échelle de la ferme avec ses infrastructures ou à l'échelle du bassin versant (Gómez-Delgado, 2010). Néanmoins, les arbres d'ombrage atténuent le ruissellement et l'érosion par un facteur de près de deux par rapport aux parcelles de plein soleil, même si cela représente peu à l'échelle du bassin versant sur ce genre de sols.

\section{Applications pratiques \\ La faible érosion laminaire en parcelles sur andisols et le rôle important des routes et des bordures de parcelles dans la production de sédiments conduisent à recommander la construction de canalisations en béton le long des chemins afin de détourner le ruissellement vers les parcelles où il pourra être infiltré. Il faut aussi protéger en priorité les berges de rivières. Quel que soit le contexte pédolo- gique de la ferme, nous recommandons d'effectuer une étude hydrologique rapide avant de choisir les meilleures mesures contre l'érosion (Gómez-Delgado, 2010).}

\section{Fertilisation, ombrage et rendement}

Selon les résultats préliminaires de Van den Meersche et al. (en préparation), la quantité d'azote recyclée par l'écosystème est plus élevée sous ombrage qu'en plein soleil. Les arbres d'ombrage étudiés ici étaient des érythrines. Le taux de recyclage est plus élevé si les érythrines sont émondées régulièrement (environ 150 à $300 \mathrm{~kg} \mathrm{~N}$ recyclé/ha/an) que si elles poussent librement ( $<70 \mathrm{~kg} \mathrm{~N}$ recyclé/ha/an). Cinq mois après l'élagage de l'érythrine, plus de $40 \%$ de l'azote des résidus d'élagage persistent dans la litière et le sol et $23 \%$ se retrouvent dans les caféiers. Aussi, dans le système basé sur la taille, l'élagage apparaît comme la voie essentielle de transfert de l'azote de l'érythrine au caféier. 
Sous ombrage et sur andisols, nous avons observé que le sol peut être laissé sans fertilisation pendant environ cinq ans sans chute du rendement en café. Ceci a été observé lors de la manipulation des apports d'engrais $(0,110$ ou $230 \mathrm{~kg} \mathrm{~N} / \mathrm{ha} / \mathrm{an})$. Lazote total du sol n'a pas été affecté dans le traitement faiblement fertilisé, alors que l'azote minéral a baissé d'un facteur trois dans le traitement faiblement fertilisé par rapport au traitement fortement fertilisé. La production alternée (forte-faible) sur deux ans, ou biennialité, du rendement est plus élevée en plein soleil (Schnabel et al., 2018).

\section{Applications pratiques}

La grande capacité de stockage d'azote des andisols permet de conserver de bons rendements en café pendant plusieurs années, malgré des apports réduits d'engrais (ici la moitié du niveau actuel de $200 \mathrm{~kg} / \mathrm{ha}$ ), voire des apports nuls. Cette information est utile lorsque les conditions économiques sont défavorables dans le contexte de fluctuations des prix du café. À plus long terme, il est prometteur de prolonger les études d'optimisation des apports d'engrais pour des questions de rentabilité économique et d'impact sur l'environnement. Cependant, les exigences en matière d'engrais dépendent beaucoup du type de sol. La grande variabilité des sols à l'échelle de la ferme pourrait inciter à une agriculture de précision, consistant à ajuster la fertilisation aux conditions micro-locales, afin de réduire les dépenses totales et la contamination des eaux en nitrates.

\section{Fertilisation et flux de $\mathrm{N}_{2} \mathrm{O}$ du sol}

Van den Meersche et al. (en préparation) ont mesuré les flux de $\mathrm{N}_{2} \mathrm{O}$ du sol dans les parcelles de plein soleil pendant une année en utilisant des chambres dynamiques (mesures continues). Les parcelles testées ont reçu des apports d'azote contrastés (0 vs $230 \mathrm{~kg} \mathrm{~N} / \mathrm{ha} . \mathrm{an}$ ), ceci dès quatre ans avant la mesure. Dans les parcelles non fertilisées, les flux de $\mathrm{N}_{2} \mathrm{O}$ sont restés faibles, autour de $1 \mathrm{~g} \mathrm{~N}-\mathrm{N}_{2} \mathrm{O} /$ ha/jour. Dans les parcelles fertilisées, les flux ont considérablement augmenté : de $1 \mathrm{~g} \mathrm{~N}-\mathrm{N}_{2} \mathrm{O} / \mathrm{ha} /$ jour pendant la saison sèche, jusqu'à 2-3 $\mathrm{g} \mathrm{N}-\mathrm{N}_{2} \mathrm{O} /$ ha/jour pendant la saison humide. Ils ont culminé à $15 \mathrm{~g} \mathrm{~N}-\mathrm{N}_{2} \mathrm{O} /$ ha/jour juste après l'application d'engrais avec un pic étroitement lié aux événements pluvieux et pouvant durer jusqu'à deux mois. Cependant, moins de $1 \%$ de l'azote apporté par l'engrais a été libéré sous forme de $\mathrm{N}_{2} \mathrm{O}$. Après la pluie, les taux d'infiltration élevés éliminent les nitrates, et entraînent une diminution des conditions redox. Ainsi, l'azote $(\mathrm{N})$ est plutôt susceptible d'être soustrait au système sol que d'être libéré sous forme de $\mathrm{N}_{2} \mathrm{O}$ dans l'atmosphère.

\section{Applications pratiques}

Nous avons mesuré des émissions de $\mathrm{N}_{2} \mathrm{O}$ sensiblement inférieures à celles calculées à partir des valeurs standard du GIEC* (protocole C-Neutral standard). En outre, une optimisation de l'apport d'engrais, avec de moindres quantités et des fréquences d'apport plus élevées, aiderait encore à diminuer les émissions de $\mathrm{N}_{2} \mathrm{O}$ et réduirait considérablement la lixiviation potentielle des nitrates.

* Groupe d'experts intergouvernemental sur l'évolution du climat : https://www.ipcc.ch/ 


\section{Un ombrage adéquat atténue le complexe des maladies foliaires sans affecter le rendement}

Allinne et al. ont mis en place une étude factorielle avec deux niveaux d'exposition (ombrage vs plein soleil) et deux niveaux de protection phytosanitaire (conventionnel vs protection maximale), dans laquelle on a mesuré la sévérité (en \% de la surface foliaire affectée) de cinq bioagresseurs foliaires : Mycena citricolor (Ojo de gallo ou american leaf spot), Hemileia vastatrix (Roya ou rouille), Cercospora caffeicola (Cercospora, cercosporiose), Leucoptera coffealia (mineuse) et Phoma costaricensis (Phoma). L'expérimentation a duré trois ans (2012-2014), incluant l'épidémie régionale historique de rouille de 2012.

La protection maximale a réduit considérablement l'impact des maladies. Quelle que soit l'année considérée, l'exposition ombrage ou plein soleil n'a pas eu d'effet sur les impacts de la rouille ou de la mineuse. En revanche, l'ombrage a réduit l'impact de la cercosporiose. Mais il a aggravé celui de Mycena citricolor (Ojo de gallo). Globalement, lorsque l'on prend en compte les effets cumulés de l'ensemble du cortège des maladies et ravageurs foliaires, l'ombrage a réduit significativement la sévérité du complexe de bioagresseurs de 10 à $20 \%$. L'ombrage a réduit à la fois la charge en fruits des caféiers, mais aussi les pertes de rendement attribuables aux maladies; ce qui s'est finalement compensé. Un rendement conservé avec des besoins réduits en pesticides-fongicides confère un réel intérêt des arbres d'ombrage dans la culture de caféiers.

\section{Applications pratiques}

Si l'on considère l'ensemble du cortège de bioagresseurs foliaires du caféier, l'ombrage réduit de 10 à $20 \%$ la sévérité sans affecter le rendement. Un ombrage adéquat peut réduire les besoins en pesticides-fongicides et améliorer la rentabilité de la culture de caféiers.

\section{L'indice foliaire (LAI) du caféier, un indicateur des services écosystémiques}

Une reconstruction des séries chronologiques de LAI (2001-2011) a été réalisée grâce à deux calibrations successives :

- la calibration du Normalized difference vegetation index (NDVI) provenant d'une image satellite à haute résolution via le LAI vrai mesuré au sol sur chacune des couches (arbres d'ombrage et caféiers);

- la calibration du NDVI obtenu par Moderate resolution imaging spectroradiometer (Modis) (Le Maire et al., 2014; Taugourdeau et al., 2014) avec l'image à haute résolution calibrée.

Le LAI du caféier varie de façon saisonnière entre 2,4 et $4,4 \mathrm{~m}^{2}$ feuille $/ \mathrm{m}^{2}$ sol avec un maximum vers la fin de la saison humide, une forte baisse au cours de la saison sèche, un minimum après l'élagage annuel du caféier, un rétablissement durant la saison des pluies suivante et un second minimum pendant la période de remplissage du grain. Avec Modis, nous avons également détecté des variations interannuelles 
significatives de LAI provenant de l'élagage annuel ou de la rénovation des parcelles. Le LAI des arbres d'ombrage, les érythrines, était en moyenne annuelle de $0,67 \mathrm{~m}^{2}$ feuille $/ \mathrm{m}^{2}$ de sol.

Nous avons corrélé les séries chronologiques du LAI du caféier avec les registres agricoles afin d'examiner les répercussions de la gestion des parcelles sur le LAI et sur certains services écosystémiques, en particulier le rendement et le ruissellement de surface. La fertilisation en azote est ajustée annuellement par l'agriculteur, elle reste le meilleur prédicteur de rendement. Mais en combinant la fertilisation en azote avec le LAI de six mois de l'année, la prédiction s'améliore. Le LAI est donc un co-prédicteur important du rendement. Ce modèle peut s'utiliser pour la cartographie des rendements régionaux et pour la reconstruction des séries temporelles de rendements passés.

Lindice foliaire a aussi été utilisé dans la modélisation des services hydrologiques. Lorsqu'il est doublé de 3,8 à 7,6, l'évapotranspiration est augmentée de $60 \%$, le ruissellement superficiel diminue de $1 \%$, le débit de la rivière se réduit de $17 \%$ et la quantité d'eau qui traverse l'aquifère est réduite de $20 \%$ (Taugourdeau et al., 2014).

\section{Applications pratiques}

Le LAI est un indicateur de rendement au niveau de la ferme, mais aussi au niveau régional. La télédétection du LAI fonctionne également pour évaluer des pertes de rendement associées aux pertes de feuillage. C'est une technique très prometteuse pour suivre la rouille et l'american leaf spot. Elle pourrait être préconisée dans les programmes d'alerte qui se sont beaucoup développés récemment, suite à l'attaque historique de rouille en 2012. Le LAI peut être aussi utilisé pour simuler des services hydrologiques.

\section{Les racines du caféier représentent $50 \%$ de la biomasse}

Les racines de caféier poussent jusqu'à $4 \mathrm{~m}$ de profondeur. La biomasse souterraine du caféier peut être estimée allométriquement.

Nous avons estimé la biomasse et la productivité primaire nette (NPP) de la parcelle (caféiers et arbres d'ombrage érythrine), en tenant compte des racines profondes et de la position des plants de caféier par rapport aux arbres. La croissance du cep et des racines, ainsi que la biomasse, le renouvellement et la décomposition des racines ont été évaluées. Les anneaux de croissance ont été étudiés à la base du cep sur des gammes de tailles de plants de caféiers. La biomasse racinaire et la densité de racines fines ont été mesurées dans des tranchées jusqu'à une profondeur de $4 \mathrm{~m}$. Le renouvellement des racines fines a été mesuré par carottage séquentiel du sol sur 0-30 cm pour tenir compte de l'hétérogénéité du sous-sol agroforestier. Ceci a été réalisé pendant un an et à différents endroits (en plein soleil ou sous les arbres, sur le rang et dans l'interrang) (Defrenet et al., 2016).

Des relations allométriques ont été proposées pour calculer la biomasse et la productivité primaire nette des parties pérennes à l'échelle de la parcelle. Des relations linéaires entre la surface basale au collet et la biomasse des compartiments pérennes 
(ceps, racines grossières, etc.) permettent d'estimer aisément le stock de carbone dans la biomasse des plantations de caféier.

Le rayon au collet du cep augmente jusqu'à 2,5 mm/an (stabilisé entre 12 et 44 ans). $92 \%$ de la biomasse racinaire du caféier est située sur une profondeur de 1,5 m, et seulement $8 \%$ jusqu'à $4 \mathrm{~m}$. La biomasse des racines pérennes est de $16 \mathrm{t} / \mathrm{ha}$ et leur productivité primaire nette de 1,3t/ha/an. La biomasse des racines fines (sur une profondeur de 0 à $30 \mathrm{~cm}$ ) est deux fois plus élevée dans le rang que dans l'interrang. La biomasse des racines fines est de 2,29t/ha, soit $12 \%$ de la biomasse racinaire totale,. Leur productivité primaire nette est de $2,96 \mathrm{t} / \mathrm{ha} / \mathrm{an}$, soit $69 \%$ de la productivité primaire nette totale de la racine. Le taux de renouvellement des racines fines est de 1,3 fois/an.

Le système racinaire de caféier représente $49 \%$ de la biomasse végétale totale. Un tel ratio est peut-être une conséquence de l'élagage. Il n'y a aucun effet significatif des arbres d'ombrage sur la biomasse des racines fines du caféier, ce qui suggère que le système racinaire du caféier est très compétitif dans la couche superficielle du sol (Defrenet et al., 2016).

Les anneaux de croissance sont annuels pour des caféiers en zone humide sans interruption de croissance. Létude de ces anneaux de croissance, combinée à l'inventaire de la répartition surface au collet, permet de calculer l'âge moyen d'une parcelle entière (Defrenet et al., 2016).

\section{Applications pratiques}

Les équations allométriques basées sur le diamètre au collet du caféier permettent d'estimer aisément sa biomasse souterraine et donc son stock de carbone dans la biomasse souterraine. La caractérisation de l'âge et des cohortes moyennes de la parcelle, telle que proposée ici, pourrait être très utile pour estimer la vigueur et la productivité d'une parcelle, ou pour normaliser les comparaisons habituellement effectuées entre les plantations, voire pour évaluer la valeur d'une plantation donnée sur le marché. Cet âge moyen pourrait également être utilisé pour déterminer le moment optimal auquel appliquer la rénovation.

\section{Les arbres d'ombrage augmentent l'efficience d'utilisation de la lumière du caféier, ce qui compense les pertes de productivité primaire nette}

Les arbres d'ombrage des systèmes agroforestiers affectent la répartition de la lumière (Charbonnier et al., 2013). Nos observations de terrain et la modélisation 3D montrent que la lumière sous les arbres est très variable et modifie la physiologie de la culture intercalaire (Charbonnier et al., 2017). La réduction du rayonnement photosynthétiquement actif absorbé (Apar) est, dans une certaine mesure, compensée par une augmentation de l'efficience de l'utilisation de la lumière (LUE). La différence de productivité primaire nette du caféier entre l'ombre et le plein soleil est ainsi réduite. En raison de la grande hétérogénéité spatiale des systèmes agroforestiers et du manque d'outils appropriés, les effets combinés de ces variables avaient rarement 
été analysés. Pourtant, leur étude permet de comprendre les processus physiologiques sous-jacents à la dynamique des rendements (Charbonnier et al., 2017).

Dans cette étude, la productivité primaire nette (NPP) à l'échelle du caféier et de la parcelle a été mesurée pendant deux ans. Le rayonnement absorbé (Apar) a été cartographié avec un modèle 3D (Maespa) : 35\% de la lumière disponible au-dessus des caféiers arrive au sol. Cela constitue une perte de ressource considérable (Charbonnier et al., 2013). On ne peut pas augmenter la densité des caféiers à cause des maladies, mais il est possible de couvrir le sol avec des cultures utiles. L'efficience d'utilisation de la lumière et le taux net d'assimilation ont été calculés individuellement pour chaque caféier. Bien que la lumière disponible ait été réduite de $60 \%$ en dessous des couronnes d'arbres d'ombrage, l'efficience d'utilisation de la lumière du caféier a augmenté de $50 \%$. La productivité primaire nette et le rendement des caféiers sous ombrage sont donc restés à un niveau élevé. À l'issue du projet, une base de données a été constituée. Elle regroupe biomasse et productivité primaire nette du caféier en condition de plein soleil ou d'ombrage. La variabilité de la productivité primaire nette intraparcelle du caféier est principalement expliquée par l'âge des plantes et par une compétition intraspécifique entre caféiers plutôt que par la présence d'arbres d'ombrage (Charbonnier et al., 2017).

\section{Applications pratiques}

Les modèles de transmission de lumière indiquent que $35 \%$ de la lumière disponible au-dessus des caféiers finit au sol : cette ressource importante est sous-utilisée et force au désherbage. Il serait judicieux de développer des plantes de couverture, fixatrices de $\mathrm{N}_{2}$ si possible. Mais la crainte de la compétition avec le caféier ou des serpents dans les interrangs est un frein à cela dans beaucoup de régions.

La compensation de la perte de lumière sous les arbres d'ombrage par une efficience accrue de l'utilisation de la lumière du caféier renforce la confiance dans l'utilisation d'arbres d'ombrage. Les modèles utilisés, comme Maespa, permettent de calculer la concurrence pour la lumière entre caféiers, les plus anciens ombrageant les plus jeunes, et de calculer ou d'optimiser les impacts de l'élagage et de la densité des plants de caféier sur leur productivité.

Grâce aux modèles, la lumière absorbée par les caféiers est devenue une variable continue dans l'espace et le temps. Ceci permet de mettre fin à la dichotomie ombre/ lumière longtemps utilisée pour décrire les systèmes agroforestiers. La lumière peut ainsi être calculée pour chaque plante, quelle que soit sa distance à l'arbre ou son environnement radiatif. La lumière absorbée peut être cartographiée et utilisée comme co-variable pour tout autre type d'étude agronomique dans des parcelles agroforestières hétérogènes.

\section{Pourquoi le caféier est-il épuisé après seulement quelques années de production? Le rôle de l'amidon}

La taille des caféiers est une action cruciale car elle affecte les rendements et le besoin de main-d'œuvre. Seulement, les caféiers ne sont productifs que pendant 
trois ans sur les six ans de durée de vie du rejet avec des coûts de main-d'œuvre élevés. Pourquoi une si courte période productive?

Nous avons étudié les effets de l'exposition (ombrage ou plein soleil) et de la maturité (pieds immatures de deux ans ou pieds matures de 5 ans) sur les concentrations de carbohydrates non structurels (NSC), leurs stocks dans les feuilles, tiges et ceps, ainsi que la dynamique de l'amidon au cours de la vie des caféiers. Nous avons utilisé les méthodes analytiques classiques et la spectroscopie de réflectance visible et proche infrarouge (VNIRS) (Cambou et al., soumis).

Les variables trophiques liées à l'activité de photosynthèse (azote, glucose, fructose, saccharose) diminuent depuis les feuilles vers les tiges, puis les souches. En revanche, les variables liées à la structure et aux réserves de la plante $(\mathrm{C}, \mathrm{C}: \mathrm{N}$ et amidon $)$ ont montré la tendance inverse, avec des valeurs plus élevées dans les organes à longue durée de vie comme les ceps. Les concentrations de N, glucose et fructose sont plus élevées dans les organes de deux ans que dans ceux de cinq ans. Ceci confirme un ratio source/puits plus important en l'absence du puits de fruits. Leffet de l'exposition, plein soleil ou ombrage, est faible sur les variables mesurées. La spectroscopie de réflectance visible et proche infrarouge (VNIRS) est un outil prometteur, rapide et rentable pour suivre la dynamique des carbohydrates non structurels, en particulier l'amidon.

Nos résultats montrent que le rejet (temporaire) s'épuise alors même que son cep (pérenne) accumule de l'amidon. Lépuisement du rejet provient d'une forte concurrence entre trois grands puits : les fruits, la croissance des rejets et le stockage de l'amidon dans les parties pérennes. En effet, le caféier est l'un des rares arbustes qui verse (se plie) naturellement avant de rejeter. Il illustre la fonction de «survie» des carbohydrates non structurels chez les plantes (Cambou et al., soumis).

\section{Applications pratiques}

Des variétés de caféier sélectionnées pour accumuler moins d'amidon dans leurs parties pérennes exprimeront probablement une durée de vie de leurs rejets prolongée et donc un plus grand nombre d'années productives du rejet. La VNIRS, en tant que technique de mesure à haut débit, permettra le dépistage de ces variétés.

\section{Bilan de carbone de l'écosystème}

Les fermes caféières sont-elles déjà proches de la neutralité C? Tout dépend de la méthode de calcul.

La ferme d'Aquiares (Costa Rica) a réalisé l'évaluation de ses émissions de gaz à effet de serre pour l'usine, les déchets et les engrais. Les émissions annuelles de 1100 t $\mathrm{CO}_{2}$ eq ont été compensées financièrement, ce qui était nécessaire pour l'obtention de la certification «C-Neutre» de la ferme en 2016. Cette ferme peut servir de pilote au niveau national et potentiellement au niveau régional pour l'avenir. Si les facteurs d'émission du GIEC sont utilisés, un tiers des émissions de la ferme serait dû aux émissions de $\mathrm{N}_{2} \mathrm{O}$ par l'utilisation d'engrais et presque autant aux émissions de $\mathrm{CH}_{4}$ par la fermentation de la pulpe. 
Selon les règles de certification C-Neutre (scénario 1), seuls les arbres d'ombrage en croissance, les forêts en croissance et la biomasse du bois de caféier utilisée comme énergie recyclée pour le séchage du grain de café peuvent être considérés comme des puits de carbone de la ferme. Le bois recyclé pour les séchoirs est une énergie renouvelable qui compense une bonne part des émissions de gaz à effet de serre de l'usine, des engrais $\left(\mathrm{N}_{2} \mathrm{O}\right)$ et des déchets $\left(\mathrm{CH}_{4}\right)$. Néanmoins, les caféiers et le sol restent exclus des calculs de ce scénario 1. En effet, le GIEC considère que les cultures en général sont neutres dans les échanges de gaz à effet de serre entre l'écosystème et l'atmosphère.

Que se passerait-t-il si le caféier et le sol étaient ré-évalués en tant que puits?

Le scénario 2 teste cette hypothèse et considère les caféiers non pas comme une culture annuelle standard, mais comme un petit arbre. Le scénario 2 inclut donc le stockage de carbone des caféiers en croissance, ainsi que dans la litière et le sol. Le bilan de carbone a été mesuré par Eddy-covariance des parcelles agroforestières (arbres + caféier + litière + sol) de la ferme d'Aquiares pendant huit années, pour un stockage moyen de 3,6 t C/ha/an. De ce chiffre, il faut en réalité déduire les

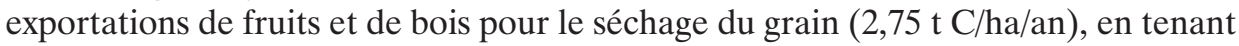
compte du fait que la combustion du bois a déjà été décomptée comme énergie renouvelable dans le calcul des émissions annuelles de 1100 t CO2eq. Au final, la séquestration de carbone par les parcelles agroforestières, en prenant les caféiers en compte, compense les émissions de l'usine + engrais + déchets. Cela indique que la ferme est virtuellement, si on accepte d'inclure les caféiers dans le décompte, déjà C-Neutre, voire même un puits de carbone.

\section{Applications pratiques}

La principale différence entre le scénario 1 (le protocole C-Neutre standard) et le scénario 2 est l'intégration des caféiers, de la litière et des sols. Le scénario 2 est avantageux pour l'agriculteur qui payerait moins de compensation carbone pour ses émissions car sa ferme se rapprocherait de la neutralité. Ceci reste également compatible avec l'objectif d'améliorer l'efficience en carbone de la ferme pour chaque année de certification successive. Cela aurait également l'avantage au niveau du pays, d'accélérer le processus de certification C-neutre des plantations de caféier et de créer éventuellement des crédits carbone à vendre en interne ou à l'étranger.

\section{Les arbres d'ombrage, un levier pour l'adaptation au changement climatique}

Nous avons étudié le potentiel de l'agroforesterie pour atténuer la température de canopée des caféiers par expérimentation sur le terrain et par modélisation. L'ouverture de la canopée des arbres d'ombrage a été évaluée par photographie hémisphérique au-dessus de chaque caféier.

Nous avons mesuré des différences de variables microclimatiques entre les parcelles à l'ombre et celles en plein soleil (Soma et al., 2017). L'ombrage réduit la température maximale de l'air de $1,7^{\circ} \mathrm{C}$. Les feuilles de caféier en plein soleil ont montré des températures jusqu'à $5^{\circ} \mathrm{C}$ supérieures à l'air environnant. La réduction de la tempé- 
rature moyenne journalière de la couche de caféiers serait de $-0,5^{\circ} \mathrm{C}$ pour le niveau bas dans la couronne à $-1,1^{\circ} \mathrm{C}$, pour le niveau élevé dans la couronne.

La modélisation permet d'ajuster l'ombrage pour des climats actuels et futurs, quelles que soient les conditions d'altitude, de pente, d'exposition ou de microclimat. Un modèle statistique simple et d'intérêt assez générique a été proposé pour prédire la température de la canopée du caféier en utilisant la température de l'air en plein soleil, l'ouverture de la canopée des arbres d'ombrage et la fraction de lumière diffuse. Un modèle agroforestier plus sophistiqué (Maespa-GO +) permet de calculer le bilan radiatif, les flux d'énergie, de $\mathrm{CO}_{2}$ et d' $\mathrm{H}_{2} \mathrm{O}$ dans des parcelles caféières hétérogènes complexes (Vezy et al., 2018).

\section{Applications pratiques}

Nous confirmons que l'agroforesterie constitue une solution intéressante pour atténuer les effets de l'élévation de la température sur le rendement, en complément d'autres stratégies comme la sélection variétale. Les outils de modélisation aideront à ajuster l'ombrage aux conditions locales (altitude, pente, exposition et rayonnement).

\section{Conclusions}

Les résultats interdisciplinaires obtenus à long terme dans une ferme caféière en milieu réel, montrent que la gestion du caféier pourrait être plus efficace à l'avenir pour l'énergie, l'eau, les nutriments et les services écosystémiques en général. Un meilleur couplage entre la recherche, la vulgarisation, les projets de développement et l'expérience des agriculteurs est recommandé pour orienter l'investissement, dans l'intérêt de la rentabilité économique et de l'environnement. En raison de ses nombreuses vertus confirmées ici, l'ombrage est à recommander : il est préférable de l'ajuster pour chaque ferme ou même pour chaque condition de terrain et la modélisation peut appuyer cet effort.

De plus, il est recommandé que les projets de développement ne se basent pas uniquement sur des règles générales, mais consacrent une partie de leur budget aux vérifications locales de ces règles. Un minimum d'investissement dans l'expertise scientifique au départ des projets devrait améliorer leur impact a posteriori.

\section{Hemerciements}

Notre étude fait partie de l'observatoire Coffee-Flux, développé par le Cirad, le Catie et Cafetalera Aquiares. Le site Coffee-Flux appartient au réseau d'observatoires SOERE F-ORE-T qui est soutenu annuellement par Ecofor, Allenvi et l'infrastructure nationale de recherche ANAEE-F (http://www.anaee-france.fr/fr/). Coffee-Flux a été soutenu par le projet européen Cafnet (EuropAid/121998/C/G), le projet Ecosfix (ANR-2010-STRA-003-01), le projet Safse (Cirad/IRD) et le projet Macacc (ANR-13-Agro-0005). Nous sommes très redevables à la famille Barquero et à Alexis Perez pour une assistance sur le terrain et à Patricia Leandro pour les installations de laboratoire du Catie. 


\section{- Bibliographie}

Benegas L., Ilstedt U., Roupsard O., Jones J., Malmer A., 2014. Effects of trees on infiltrability and preferential flow in two contrasting agroecosystems in Central America. Agriculture, ecosystems \& environment, 183(0): 185-196.

Cambou A., Barthès B., Clement-Vidal A., Thaler P., Charbonnier F., Van Den Meersche K., Avelino J., DavrieuxvF., Labouisse J.-P., Aguilar M.H., De Melo Virginio Filho E., Deleporte P., Brunet D., Gay A., Lehner P., Roupsard O., subm. Why is Coffea arabica exhausted after just a few years of yield? Starch sink in perennial stumps competes but ensures survival. Tree Physiology. (Submitted 26 ${ }^{\text {th }}$ of April 2017, rejected, to be re-submitted).

Charbonnier F., Le Maire G., Dreyer E., Casanoves F., Christina M., Dauzat J., Eitel J.U.H., Vaast P., Vierling L.A., Roupsard O., 2013. Competition for light in heterogeneous canopies: Application of Maestra to a coffee (Coffea arabica L.) agroforestry system. Agricultural and forest meteorology, 181: 152-169.

Charbonnier F., Roupsard O., Le Maire G., Guillemot J., Casanoves F., Lacointe A., Vaast P., Allinne C., Audebert L., Cambou A., Clement-Vidal A., Defrenet E., Duursma R.A., Jarri L., Jourdan C., Khac E., Leandro P., Medlyn B.E., Saint-Andre L., Thaler P., Van Den Meersche K., Aguilar A.B., Lehner P., Dreyer E., 2017. Increased light-use efficiency sustains net primary productivity of shaded coffee plants in agroforestry system. Plant cell and environment, 40(8): 1592-1608.

De Melo Virginio Filho E., Arrieta S., Escobedo Aguilar A., Rapidel B., Roupsard O., 2015. Validación de Piloto de Sistemas Agroforestales en el ámbito de la Nama-Café Costa Rica (Propuesta para ejecución en 2016). Annexo 2. Catie, Cirad, Fundecooperacion, Icafe, MAG. 30 p., Diseño piloto de sistemas agroforestales en el ámbito de la Nama-Café Costa Rica, 16 p.

Defrenet E., Roupsard O., Van Den Meersche K., Charbonnier F., Pastor Pérez-Molina J., Khac E., Prieto I., Stokes A., Roumet C., Rapidel B., De Melo Virginio Filho E., Vargas V.J., Robelo D., Barquero A., Jourdan C., 2016. Root biomass, turnover and net primary productivity of a coffee agroforestry system in Costa Rica: effects of soil depth, shade trees, distance to row and coffee age. Annals of botany, 118(4): 833-851.

Gómez-Delgado F., 2010. Hydrological, ecophysiological and sediment processes in a coffee agroforestry basin: combining experimental and modelling methods to assess hydrological environmental services. Thèse de doctorat, Montpellier, SupAgro; École doctorale Systèmes intégrés en biologie, agronomie, géosciences, hydrosciences, environnement. Montpellier, $254 \mathrm{p}$.

Gómez-Delgado F., Roupsard O., Le Maire G., Taugourdeau S., Perez A., Van Oijen M., Vaast P., Rapidel B., Harmand J.-M., Voltz M., Bonnefond J.-M., Imbach P., Moussa R., 2011. Modelling the hydrological behaviour of a coffee agroforestry basin in Costa Rica. Hydrology and earth system sciences, 15(1): 369-392.

Hasselquist N.J., Benegas L., Roupsard O., Malmer A., Ilstedt U., 2018. Canopy cover effects on local soil water dynamics in a tropical agroforestry system: Evaporation drives soil water isotopic enrichment. Hydrological processes, 32(8): 994-1004.

Isaac M.E., Martin A.R., de Melo Virginio Filho E., Rapidel B., Roupsard O., Van den Meersche K., 2017. Intraspecific trait variation and coordination: root and leaf economics spectra in coffee across environmental gradients. Frontiers in plant science, 8: 1196.

Kim J.H., Fourcaud T., Jourdan C., Maeght J.L., Mao Z., Metayer J., Meylan L., Pierret A., Rapidel B., Roupsard O., de Rouw A., Sanchez M.V., Wang Y., Stokes A., 2017. Vegetation as a driver of temporal variations in slope stability: The impact of hydrological processes. Geophysical research letters, 44: 4897-4907.

Kinoshita R., Roupsard O., Chevallier T., Albrecht A., Taugourdeau S., Ahmed Z., Van Es H.M., 2016. Large topsoil organic carbon variability is controlled by Andisol properties and effectively assessed by VNIR spectroscopy in a coffee agroforestry system of Costa Rica. Geoderma, 262: 254-265.

Le Maire G., Rançon F., Charbonnier F., Roupsard O., 2014. Characterizing the structure of a coffee agroforestry systems in Costa Rica. Poster, In: Pleiades days. 1-3 avril 2014. Toulouse, France. 
Martin A.R., Rapidel B., Roupsard O., Van Den Meersche K., Virginio E.D., Barrios M., Isaac M.E., 2017. Intraspecific trait variation across multiple scales: the leaf economics spectrum in coffee. Functional ecology, 31(3): 604-612.

Prieto I., Roumet C., Cardinael R., Dupraz C., Jourdan C., Kim J.H., Maeght J.L., Mao Z., Pierret A., Portillo N., Roupsard O., Thammahacksa C., Stokes A., 2015. Root functional parameters along a land-use gradient: evidence of a community-level economics spectrum. Journal of ecology, 103(2): 361-373.

Roupsard O., 2015. La utilización de modelos para apoyar la validación de Innovaciones Agroforestales, In : Diseño de Piloto de Sistemas Agroforestales en el ámbito de la Nama-Café Costa Rica-Catie-Cirad-Fundecooperación-Icafe-MAG. Catie, 09 de Diciembre de 2015, NamaCafé Costa Rica Oral presentation.

Roupsard O., Van Den Meersche K., Alline C., Vaast P., Rapidel B., Avelino J., Jourdan C., Le Maire G., Bonnefond J.M., Harmand J.M., Dauzat J., Albrecht A., Chevallier T., Gómez-Delgado F., Charbonnier F., Benegas L., Welsh Unwala K.E., Kinoshita R., Vezy R., Perez Molina J.P., Kim J., Defrenet E., S T., Nespoulous J., Cambou A., Soma M., Mages C., Schnabel F., Prieto Aguilar I., Picart D., Duthoit M., Rocheteau A., Do F., De Melo Virginio Filho E., Moussa R., Le Bissonnais Y., Sanchez-Murillo R., Roumet C., Stokes A., Vierling L., Eitel J., Dreyer E., Saint-André L., Malmer A., Loustau D., Isaac M.E., Martin A., Priémé A., Elberling B., Madsen M., Robelo A., Robelo D., Borgonovo C., Lehner P., Ramirez L.G., Jara M., Acuna Vargas R., Barquero A., Fonseca C., Gay F., 2017. Eight years studying ecosystem services in a large coffee agroforestry observatory. Practical applications for the farmers, In: World Coffee summit. $31^{\text {st }}$ May- $3^{\text {rd }}$ June 2017. El Salvador. Invited oral presentation.

Sánchez-Murillo R., Birkel C., Welsh K., Esquivel-Hernández G., Corrales-Salazar J., Boll J., Brooks E., Roupsard O., Sáenz-Rosales O., Katchan I., Arce-Mesén R., Soulsby C., AraguásAraguás L.J., 2016. Key drivers controlling stable isotope variations in daily precipitation of Costa Rica: Caribbean Sea versus Eastern Pacific Ocean moisture sources. Quaternary science reviews, 131(Part B): 250-261.

Schnabel F., Virginio E.D., Xu S., Fisk I.D., Roupsard O., Haggar J., 2018. Shade trees: a determinant to the relative success of organic versus conventional coffee production. Agroforestry systems, 92(6): 1535-1549.

Soma M., Vezy R., Deswarte C., De Melo Virginio Filho E., Guilioni L., Le Maire G., Avelino J., Roupsard O., 2017. A simple model to predict the effect of Agroforestry on coffee canopy temperature. (en préparation) Agricultural and forest meteorology.

Taugourdeau S., Le Maire G., Avelino J., Jones J.R., Ramirez L.G., Quesada M.J., Charbonnier F., Gomez-Delgado F., Harmand J.-M., Rapidel B., Vaast P., Roupsard O., 2014. Leaf area index as an indicator of ecosystem services and management practices: An application for coffee agroforestry. Agriculture ecosystems and environment, 192: 19-37.

Vezy R., Christina M., Roupsard O., Nouvellon Y., Duursma R., Medlyn B., Soma M., Charbonnier F., Blitz-Frayret C., Stape J.L., Laclau J.P., Virginio E.D., Bonnefond J.M., Rapidel B., Do F.C., Rocheteau A., Picart D., Borgonovo C., Loustau D., Le Maire G., 2018. Measuring and modelling energy partitioning in canopies of varying complexity using MAESPA model. Agricultural and forest meteorology, 253: 203-217.

Welsh K., Boll J., Sanchez-Murillo R., Roupsard O., 2018. Isotope hydrology of a tropical coffee agroforestry watershed: Seasonal and event-based analyses. Hydrological processes, 32(13) : 1965-1977. 
Chapitre 3

\title{
Régulation des bioagresseurs du caféier par le couvert arboré au Costa Rica
}

\author{
Allinne C., Boudrot A., De Melo E., Granados E., Merle I., \\ Pico J., Vonthron S. et Avelino J.
}

Résumé. Dans les systèmes agroforestiers, l'ombrage peut avoir des effets synergiques ou, au contraire, antagonistes sur l'expression des dégâts du complexe de bioagresseurs et des dommages associés. Les travaux présentés ont été réalisés dans des systèmes agroforestiers à base de caféiers au Costa Rica. Ils ont pour but d'illustrer les effets des interactions de l'ombrage à différents niveaux : avec le climat et l'environnement; au sein même du cycle de vie d'un bioagresseur; entre bioagresseurs du complexe parasitaire; entre auxiliaires et bioagresseurs. L'effet de l'ombrage sur la fourniture de plusieurs services écosystémiques est également illustré. Nos résultats montrent que l'ombrage augmente la dispersion du pathogène Hemileia vastatrix, responsable de la rouille quand il pleut, alors qu'il la réduit quand il fait sec. Lombrage favorise le processus pré-infectieux et la colonisation du caféier par la rouille, ainsi que sa régulation par son ennemi naturel, le champignon Lecanicillium lecaniii. Les effets de l'ombrage sont contraires selon le bioagresseur foliaire (deux basidiomycètes, deux ascomycètes et une larve de lepidoptère). Une approche «multipest» montre qu'un ombrage optimal permet de réduire l'impact total du cortège de bioagresseurs. Enfin, l'ombrage peut avoir des effets antagonistes selon les services écosystémiques, comme en réduisant directement la production mais en augmentant la régulation des bioagresseurs. L'étude des compromis de l'ensemble de ces services doit être réalisée afin de concevoir des systèmes permettant d'optimiser la régulation des bioagresseurs par l'intermédiaire l'ombrage.

\begin{abstract}
The shade cover on agroforestry systems can have synergic or antagonistic effects on the pest and disease ("bioagressor") injury profile and the associated damages. The present study has been carried out in coffee-based agroforestry systems in Costa Rica and aims to illustrate the effects of shade interactions at different levels: with climate/environment, within the life cycle of a bioagressor, between bioagressors of the parasitic complex, and/or between bioagressors and their natural enemies. The impact of shading on the provision of ecosystem services is also illustrated. Our results show that under shade, the dispersion of coffee rust is increased under rainy conditions, while it is reduced under dry
\end{abstract}


conditions. Shade promotes the pre-infectious process and the colonization of coffee by rust and at the same time its regulation by its natural enemy, Lecanicillium lecaniii. These effects are opposite according to the foliar pests and diseases, and our multipest approach makes it possible to demonstrate that optimal shading allows the reduction of the overall impact of foliar pests and diseases. Lastly, shading can have antagonistic effects depending on the ecosystem services, such as crop production and pest and disease regulation, and the study of trade-offs between all these services must be carried out in order to design systems which optimize pest and disease regulation via shading.

\section{Introduction}

La présence d'arbres dans la parcelle ou dans le paysage peut avoir des répercussions sur l'état sanitaire de la culture. Les effets de cette présence varient en fonction des espèces d'arbres, des bioagresseurs ou de leurs ennemis naturels, de leurs traits d'histoire de vie et des environnements physiques (Ratnadass et al., 2012).

La figure 3.1 illustre de manière simplifiée les différentes voies d'action de l'ombrage sur les composantes biotiques du système : les cortèges de bioagresseurs et de leurs ennemis naturels, ainsi que les caféiers (Avelino et al., 2011; Damatta, 2004;

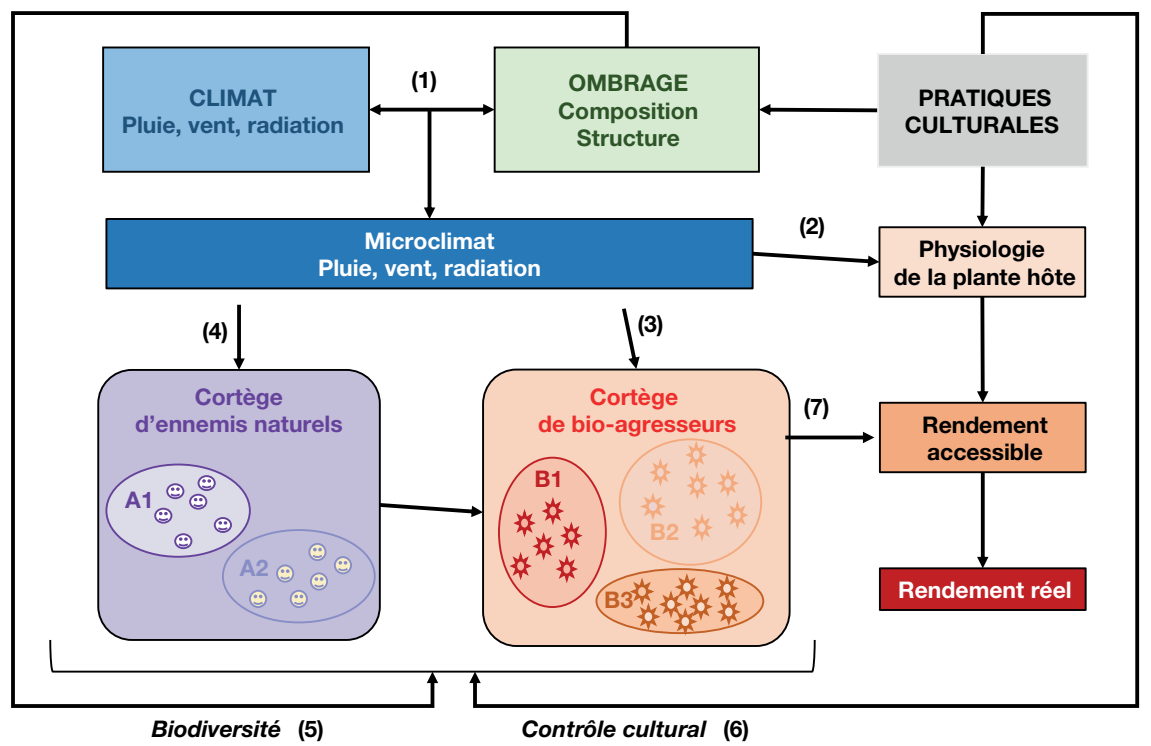

Figure 3.1. Représentation des différentes voies d'action de l'ombrage sur le cortège de bioagresseurs, les auxiliaires de culture et le rendement dans les systèmes agroforestiers à base de caféiers.

Déterminée par les pratiques, la présence d'arbres d'ombrage en interaction avec le climat détermine un microclimat (1). Ce microclimat affecte la physiologie du caféier (croissance, développement et phénologie) (2) qui détermine le rendement accessible (Rabbinge et al., 1989), c'est-à-dire sans pertes liées aux bioagresseurs. Le microclimat impacte aussi le développement des bioagresseurs (3) et leurs ennemis naturels (4). Lombrage peut également avoir un impact direct sur la présence de bioagresseurs et de leurs ennemis naturels en jouant le rôle d'hôte alternatif (5). Les pratiques influencent directement ces populations par les méthodes de contrôle utilisées (6). Enfin, l'impact des bioagresseurs, c'est-à-dire les pertes de rendement associées à l'ensemble des dégâts occasionnés par le cortège, détermine le rendement final (7). 
Muller et al., 2009; Schroth et al., 2000; Staver et al., 2001). Les effets peuvent être complexes. Il est possible d'observer des effets antagonistes à différents niveaux :

- des interactions avec l'environnement, lorsque l'ombrage conduit à réguler un bioagresseur, c'est-à-dire à contrôler son développement, mais en favorise un autre; - au sein même du cycle de vie de certains bioagresseurs, en favorisant ou en défavorisant des processus physiologiques;

- sur diverses espèces de bioagresseurs et d'ennemis naturels;

- à une échelle plus large, il peut exister des effets antagonistes des arbres d'ombrage sur la fourniture de plusieurs services écosystémiques, notamment la régulation des bioagresseurs et la production agricole.

Le bilan général de ces effets est donc très difficile à définir a priori. En s'appuyant sur l'étude des systèmes agroforestiers à base de caféiers du Costa Rica et son cortège de bioagresseurs et de leurs ennemis naturels, nous avons développé différentes approches afin de :

- mettre en évidence d'éventuelles interactions entre l'ombrage et l'environnement; - mieux comprendre les effets de l'ombrage sur les composantes biotiques des systèmes ;

- identifier d'éventuels compromis entres services écosystémiques.

Dans ce chapitre, nous nous appuyons sur ces différentes études afin d'illustrer comment l'ombrage, en interaction avec d'autres composantes du système biophysique, peut réguler les bioagresseurs du caféier par des voies d'action différentes peuvant être parfois antagonistes.

\section{Ombrage et régulation des bioagresseurs}

\section{Interactions de l'ombrage avec l'environnement, effets de l'ombrage sur la dispersion d'Hemileia vastatrix}

Au sein du système agroforestier, les interactions sont complexes et nous supposons qu'elles peuvent influencer le développement des bioagresseurs et l'expression des dégâts (symptômes physiologiques provoqués par les bioagresseurs sur la plante) et des dommages (pertes de rendement induites par les dégâts). Il peut exister une interaction entre l'ombrage, le climat et l'environnement, c'est-à-dire qu'en fonction de la présence ou pas d'arbre d'ombrage, le climat influence de manière différente le développement des bioagresseurs.

Les travaux présentés ici, publiés en 2016 par Boudrot et al., visent à étudier l'effet de l'ombrage sur la dispersion de la rouille orangée en comparant deux modalités d'ombrage :

- dans la première, sont associées deux espèces végétales d'ombrage, Erythrina poeppigiana et Chloroleucon eurycyclum;

- dans la seconde, la dispersion est évaluée sans ombrage, en plein soleil.

Pour étudier la dispersion de la rouille orangée, des pièges à spores Burkard ont été utilisés.

Nous avons mis en évidence des interactions entre l'ombrage et les conditions météorologiques pour la dispersion de H. vastatrix. Quand il pleut, les gouttes de 
pluie, interceptées par les arbres d'ombrage, s'accumulent sur les feuilles. Cette eau est ensuite redistribuée dans la parcelle de caféiers sous forme de gouttes d'eau à plus forte énergie cinétique (Van den Meersche et al., en révision). Limpact des gouttes sur les feuilles de caféier libère, pour cette raison, plus de spores sous ombrage qu'en plein soleil. Les rafales de vent annulent cet effet, probablement en empechant l'eau de s'accumuler sur les feuilles des arbres d'ombrage. Quand il ne pleut pas, les rafales de vent favorisent la dispersion des spores en plein soleil, alors qu'elles n'ont aucun effet dans les conditions d'ombrage, probablement parce que la canopée des arbres intercepte le vent.

Ces résultats mettent en exergue l'importance de la gestion des arbres d'ombrage en fonction de la météorologie pour contrôler la dispersion des spores d'H. vastatrix : tailler les arbres d'ombrage pendant les périodes pluvieuses et les laisser s'étendre pendant les périodes sèches. Nos résultats permettent aussi d'identifier des traits d'intérêt des arbres d'ombrage pour éviter la dispersion d'H. vastatrix. En effet, il faut des arbres qui ne permettent pas une augmentation de l'énergie cinétique des gouttes d'eau : petite taille, grande surface spécifique des feuilles et feuilles découpées. L’approche développée dans cette étude, basée sur l'étude des traits fonctionnels des arbres d'ombrages pourrait être étendue à d'autres processus du cycle de vie d'H. vastatrix.

\section{Effets contraires de l'ombrage au cours du cycle de vie d'un bioagresseur}

Il s'agit de comprendre les effets contraires de l'ombrage pendant le cycle de vie d'un bioagresseur : l'incidence et la sévérité de la rouille orangée, la sporulation du pathogène Hemileia vastatrix et le parasitisme de Lecanicillium lecanii sur les lésions de rouille.

Lombrage peut avoir des effets positifs ou négatifs selon les composantes affectées durant le cycle de vie d'un bioagresseur. Une étude réalisée en 2013 et 2014 sur la rouille du caféier illustre ce phénomène (Pico, 2014). Au cours de cette étude réalisée dans l'essai d'agroforesterie du Catie à Turrialba, et de gestion de la rouille (tab. 3.1) :

- l'incidence (pourcentage de feuilles atteintes, qui renseigne sur la dissémination de la maladie);

- la sévérité (pourcentage de surface foliaire atteinte, qui renseigne sur le développement de la maladie) de la rouille;

- le parasitisme de la rouille par le champignon entomopathogène et mycoparasite Lecanicillium lecanii.

L'ombrage tend à augmenter le degré d'infestation de la rouille orangée, en favorisant à la fois sa propagation et son développement. En effet, les processus pré-infectieux, mesurés par l'incidence (nombre de succès de l'infection), ont été favorisés (germination et pénétration). La colonisation a également été favorisée, comme le montre l'augmentation de la sévérité. Ces tendances ont déjà été rapportées lors d'une étude où la charge fruitière en baies de café a été homogénéisée afin d'en supprimer l'effet (López-Bravo et al., 2012), ce qui est le cas aussi ici. 
Tableau 3.1. Description des traitements utilisés dans l'essai d'agroforesterie du Catie à Turrialba afin d'étudier l'effet de l'ombrage sur l'incidence et la sévérité de la rouille orangée du caféier.

\begin{tabular}{cccc}
\hline Traitement & Type d'ombrage & Type de gestion & Niveau d'intrants \\
\hline $\mathrm{T} 1$ & $\begin{array}{c}\text { E. poeppigiana } \\
\text { C. eurycyclum }\end{array}$ & Biologique & Élevé \\
\hline $\mathrm{T} 2$ & $\begin{array}{r}\text { E. poeppigiana } \\
\text { C. eurycyclum }\end{array}$ & Biologique & Élevé \\
\hline $\mathrm{T} 3$ & $\begin{array}{l}\text { E. poeppigiana } \\
\text { C. eurycyclum }\end{array}$ & Conventionnelle & Faible \\
\hline $\mathrm{T} 4$ & Plein soleil & Conventionnelle & Faible \\
\hline $\mathrm{T} 5$ & E. poeppigiana & Conventionnelle & Faible sans fongicide \\
\hline $\mathrm{T} 6$ & Plein soleil & Conventionnelle & Faible sans fongicide \\
\hline
\end{tabular}

Par ailleurs, les effets de l'ombrage sur l'intensité de la sporulation (nombre de spores par centimètre carré de surface foliaire contaminée) sont extrêmement faibles. Une seule différence significative a été observée, avec une intensité plus élevée sous ombrage dense (E. poeppigiana et $C$. eurycyclum) par rapport à E. poeppigiana. À noter que l'intensité de sporulation diminue avec le temps dans les systèmes biologiques ou conventionnels sans fongicide. Elle augmente avec le temps dans les systèmes conventionnels avec fongicide. Cette évolution peut s'expliquer par le parasitisme de la rouille sur $L$. lecanii qui est plus intense dans les traitements biologiques et conventionnels sans fongicide. Il y a donc, dans ces traitements, un effet de régulation de l'épidémie par $L$. lecanii. Même si cet effet est tardif - il ne s'exprime qu'en seconde partie d'année, c'est-à-dire à un stade avancé du cycle de production du café et de développement de la rouille —, l'ombrage favorise l'hyperparasitisme de L. lecanii.

En résumé, l'ombrage a des effets contraires sur différents processus. Il tend à favoriser l'incidence et la sévérité de la rouille, donc les phases pré-infectieuses et de colonisation. Parallèlement, l'ombrage défavorise la sporulation, en aidant $L$. lecanii qui régule l'épidémie en fin de saison des pluies, au moment du pic de récolte du café. La question se pose de savoir si cette régulation est suffisante pour éviter des pertes.

\section{Effets contraires de l'ombrage sur différents bioagresseurs foliaires du caféier}

Lombrage peut favoriser le développement d'un bioagresseur et en défavoriser un autre. Quel est alors l'effet global de l'ombrage sur l'ensemble du cortège des bioagresseurs et son impact sur le rendement si l'on prend en compte ces effets antagonistes?

Nous traitons cette question avec une étude dans laquelle une approche originale «multipest» a été développée. Elle a pour but d'évaluer l'impact de l'ombrage sur l'ensemble du complexe de bioagresseurs foliaires du caféier (incluant les effets antagonistes des arbres d'ombrage), ainsi que sur le rendement en café. Cette approche 
«multipest» met en évidence les compromis possibles entre régulation des bioagresseurs et production. Cette expérimentation a été menée sur le site d'Aquiares (Turrialba) sur le dispositif de la plateforme CoffeeFlux (voir chapitre 2).

\section{Encadré 3.1 - Le dispositif de la plateforme CoffeeFlux}

Il s'agit d'une étude factorielle à trois facteurs (ombrage, âge et traitement pesticide ou fongicide). Au total, 80 caféiers ont été étudiés : 4 classes d'âges (de 2 à 6 ans), deux conditions (ombre et soleil), deux traitements (non traité, traité avec un pesticide) et cinq répétitions. Sur chaque caféier, six rameaux ont été marqués et suivis mensuellement. Chaque mois, nous avons mesuré le pourcentage de feuilles atteintes (incidence) et le pourcentage de surface foliaire atteinte (sévérité) par chaque maladie foliaire détectée : cinq champignons (Hemileia vastatrix, Mycena citricolor, Cercospora coffeicola, Colletotrichum coffeanum et Phoma costarricensis) et une larve mineuse de lépidoptère (Leucoptera coffeella). L'aire sous la courbe de progression de la maladie (Area under disease progress curve, AUDPC) pour chacune des deux variables, incidence et sévérité, a été calculée pour chaque année. Les mesures de rendement ont été réalisées à la récolte.

Nos résultats ont montré que l'ombrage réduit significativement la sévérité totale des maladies et ravageurs, mais il n'a pas d'effet significatif sur le rendement (Allinne et al., soumis). Même si la production en café est généralement plus élevée en plein soleil, l'ombrage permet de maintenir le rendement à un niveau acceptable même en cas d'épidémies majeures de rouille, comme en 2012. Ceci est fondamental dans un contexte de conception de systèmes de culture durables et capables qui doit prendre en compte l'équilibre entre plusieurs services écosystémiques, mais également leur stabilité dans le temps.

\section{Effets de l'ombrage sur plusieurs services écosystémiques : régulation des bioagresseurs et rendement des caféiers de base}

En agriculture, les bioagresseurs sont les principaux facteurs de réduction du rendement. Dans les systèmes de culture monospécifiques, il est possible d'en évaluer l'impact même s'il reste difficile de quantifier exactement les pertes de production associées. En revanche, dans les systèmes plurispécifiques complexes fournissant une diversité de services écosystémiques autres que la production, leur impact est moins apparent en raison des nombreuses interactions dans lesquelles ils sont impliqués. Cependant, il est essentiel de comprendre les relations entre les bioagresseurs, d'une part, et la fourniture de services écosystémiques (y compris la production agricole), d'autre part, afin de développer des agroécosystèmes durables.

Létude présentée (Allinne et al., 2016) illustre comment la biodiversité associée à l'ombrage et la gestion du système influent sur la fourniture de services écosystémiques, principalement la régulation des bioagresseurs et la production de café. Cette étude a été conduite dans des agroécosystèmes à base de caféiers au Costa Rica.

Notre étude est basée sur l'analyse de données d'enquêtes recueillies dans 107 parcelles de caféiers caractérisées par un topoclimat, un sol, des états physiologiques de production des caféiers, des pratiques culturales et un certain niveau d'incidence des 
différents bioagresseurs. Pour chacune de ces catégories de variables, nous avons réalisé une typologie des parcelles afin d'identifier des profils des principaux attributs des systèmes de culture. Les profils de dégâts ont été déterminés à partir des niveaux d'incidence de sept maladies, un insecte et deux nématodes. Les groupes obtenus (types de topoclimat, de sol, de caractéristiques physiologiques de production du café, de pratiques culturales et les profils de dégâts) sont considérés alors comme des méta-variables, décrites à partir des variables initiales. Puis, nous avons réalisé une analyse des correspondances pour évaluer les niveaux d'associations entre les profils de dégâts et les autres méta-variables descriptives des agroécosystèmes. Nous avons également intégré dans l'analyse, comme variables supplémentaires, un indicateur de la biodiversité basé sur le type d'ombrage associé au système et un indicateur du rendement basé sur une estimation du rendement avant récolte.

Quatre principaux types de systèmes de culture représentatifs d'un gradient d'intensité de gestion ont été identifiés (fig. 3.2). Le niveau de biodiversité associé aux types d'ombrage est inversement proportionnel au gradient d'intensité de gestion. Ces types de systèmes de culture vont de l'extensif (systèmes diversifiés à faible niveau d'intrants) à l'intensif (monocultures avec un fort niveau d'intrants). À chaque type, sont associés des profils de dégâts spécifiques, des niveaux de rendement différents et une certaine quantité de services écosystémiques autres que la production de

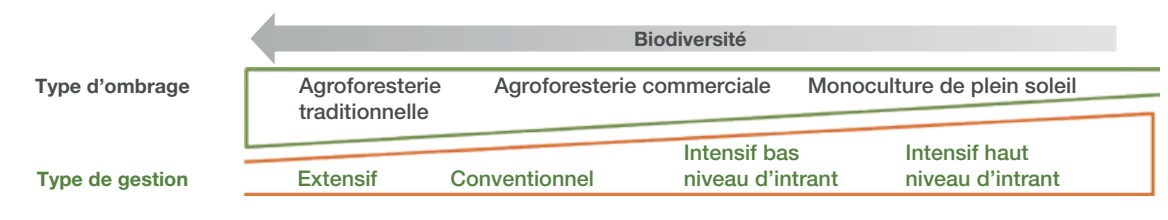

Profils de bioagresseurs
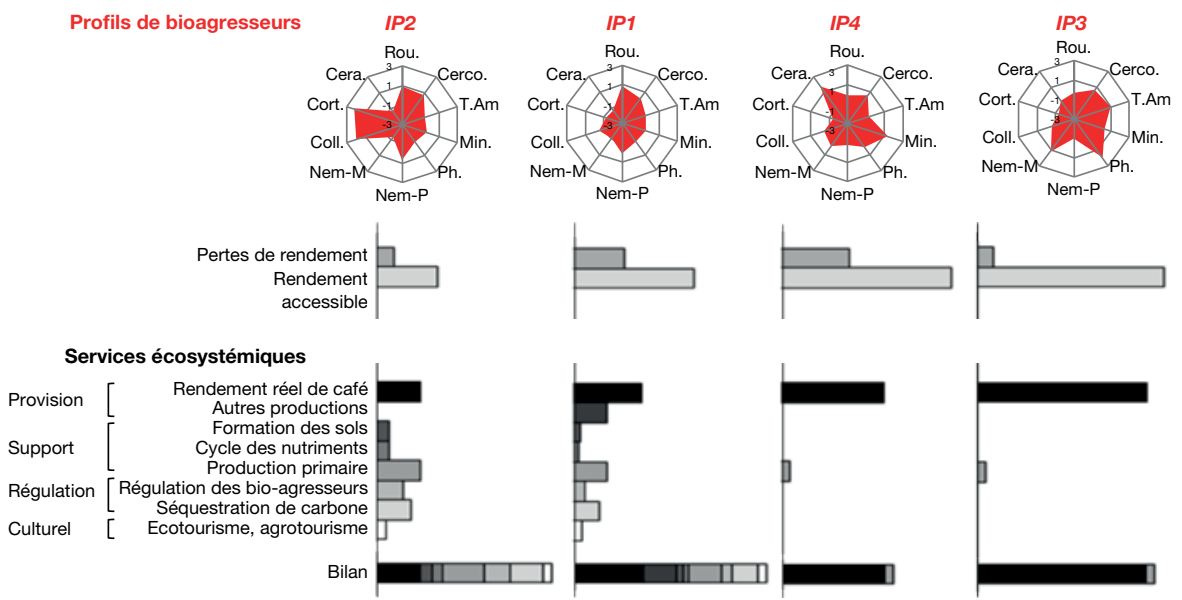

Figure 3.2. Influence de la biodiversité, associée au système de culture et à son mode de gestion, sur les profils des bioagresseurs (IP1, IP2, IP3 et IP4) et la fourniture totale de services écosystémiques.

Les bioagresseurs décrits sont : la rouille (Rou.), la cercosporiose (Cerco.), la maladie de la tache américaine (T.Am.), la mineuse des feuilles (Min.), le phoma (Ph.), les nématodes des lésions racinaires (Nem.P), les nématodes à galles (Nem.M), la mort régressive des rameaux (Coll.), la maladie de la toile d'araignée (Cort.) et le chancre du café (Cera.). Les valeurs présentées ont été centrées-réduites. (adapté de Allinne et al., 2016). 
café. Les systèmes les plus diversifiés sont associés à de faibles rendements en café, mais ils sont liés à un profil de bioagresseurs ayant peu d'impact sur la production. À l'opposé, les systèmes les plus intensifs, en monoculture, sont associés à des profils de bioagresseurs susceptibles de réduire fortement le rendement en café. Ces résultats sont synthétisés dans la figure 3.2.

Nous avons mis en évidence que les caractéristiques environnementales physiques, topoclimat et sol, étaient les principaux facteurs déterminant les profils de dégâts et les pertes de rendement associées : la présence de bioagresseurs et leur impact sur la production dépendent en premier lieu de l'environnement. Les pratiques culturales et la gestion des bioagresseurs doivent donc être d'abord adaptées à ces caractéristiques physiques. Nous avons montré que lorsque le topoclimat et le sol favorisent le développement des maladies et ravageurs pouvant conduire à de lourdes pertes de rendement, la diversification du système permet d'augmenter la fourniture de services écosystémiques, comme la production d'autres cultures, contribuant ainsi à compenser la baisse de rendement en café. Au contraire, lorsque les caractéristiques physiques de l'environnement ne sont pas favorables aux maladies et ravageurs, il est possible d'augmenter la quantité d'arbres d'ombrage et donc le nombre de services écosystémiques associés, à condition que cela ne réduise pas significativement la production de café.

\section{Conclusion}

Les études présentées montrent que les effets des arbres d'ombrage sur la régulation des bioagresseurs du caféier sont complexes et peuvent être antagonistes. D’un côté, l'ombrage augmente la dispersion de la rouille quand il pleut, et la réduit quand il fait sec. Lombrage favorise le processus pré-infectieux et la colonisation du caféier par la rouille, ainsi que le développement de son ennemi naturel, L. lecanii. Ces effets sont contraires selon le bioagresseur foliaire considéré. Seule une approche «multipest» permet, dans une condition donnée, d'établir le bilan réel de l'effet de l'ombrage sur l'ensemble du cortège de bioagresseurs. Enfin, l'ombrage peut avoir des effets antagonistes selon les services écosystémiques comme la production de café et la régulation des bioagresseurs, par exemple. L'étude des compromis de l'ensemble de ces services doit être réalisée afin de concevoir des systèmes permettant d'optimiser la régulation des bioagresseurs via l'ombrage.

\section{" Bibliographie}

Allinne C., Savary S., Avelino J., 2016. Delicate balance between pest and disease injuries, yield performance, and other ecosystem services in the complex coffee-based systems of Costa Rica. Agriculture, ecosystems and environment, 222: 1-12.

Avelino J., Ten Hoopen M., Declerck F., 2011. Ecological mechanisms for pest and disease control in coffee and cacao agroecosystems of the Neotropics. In: Ecosystem services from agriculture and agroforestry: measurement and payment. De Clerck F., Le Coq J.F., Beer J. (eds). Londres : Earthscan.

Boudrot A., Pico J., Merle I., Granados E., Vilchez S., Tixier P., Filho E.M., Casanoves F., Tapia A., Allinne C., Rice R.A., Avelino J., 2016. Shade effects on the dispersal of airborne Hemileia vastatrix uredospores. Phytopathology, 4. 
Damatta F.M., 2004. Ecophysiological constraints on the production of shaded and unshaded coffee: a review. Field crops research, 86(2-3): 99-114.

López-Bravo D.F., Virginio-Filho E.D.M., Avelino J., 2012. Shade is conducive to coffee rust as compared to full sun exposure under standardized fruit load conditions. Crop protection, 38(0): 21-29.

Muller R.A., Berry D., Avelino J., Bieysse D., 2009. Coffee diseases. In: Coffee: Growing, Processing, Sustainable production: a guidebook for growers, processors, traders, and researchers. Wintgens J.N., (eds). London: Wiley, 495-549.

Pico Rosado, J.T., 2014. Efecto de la sombra del café y el manejo sobre la incidencia, severidad, cantidad de inóculo y dispersión de Hemileia vastatrix en Turrialba, Costa Rica. Centro agronomico tropical de investigacion y ensenanza, Turrialba, Costa Rica.

Rabbinge R,Ward SA, Van Laar HH, eds. 1989. Simulation and systems management in crop protection. Wageningen: Pudoc.

Schroth G., Krauss U., Gasparotto L., Duarte Aguilar J., Vohland K., 2000. Pests and diseases in agroforestry systems of the humid tropics. Agroforestry systems, 50(3): 199-241.

Staver C., Guharay F., Monterroso D., Muschler R., 2001. Designing pest-suppressive multistrata perennial crop systems: shade-grown coffee in Central America. Agroforestry systems, 53(2): 151-170.

Van den Meersche K., Cabon M., Theriez M., Garrido A., Rapidel B., Allinne C., Rolando Cerda R., de Melo Virginio E., Roupsard O., Avelino J., soumis. Throughfall kinetic energy in coffee agroforestry systems depends on shade tree species, canopy height and leaf morphology. Agricultural and forest meteorology (soumis).

Zadoks J.C., Schein R.D., 1979. Epidemiology and plant disease management. New York: Oxford University Press. 



\title{
Chapitre 4 \\ Simulation participative pour explorer le rôle des politiques sur la production de services environnementaux au Costa Rica et au Nicaragua
}

\author{
Le Coq J.-F., Bonifazi M., Aucante M., Ferrand N., \\ Fréguin-Gresh S., Rapidel B. et Sibelet N.
}

\begin{abstract}
Résumé. Alors que les systèmes agroforestiers sont reconnus pour la diversité des services environnementaux qu'ils fournissent, la nature et l'ampleur de ces services dépendent des choix des producteurs. Différents instruments de politiques ont été proposés pour influencer les choix des pratiques des producteurs et promouvoir la production de services environnementaux. Toutefois, il n'est pas aisé d'anticiper les réponses des producteurs à ces instruments. Dans ce chapitre, nous présentons les résultats de l'application d'une méthodologie originale fondée sur une approche à la fois participative et prospective conçue et mise en ouvre avec les acteurs locaux et des producteurs afin de tester l'intérêt de certains instruments encourageant la production de services environnementaux dans leur contexte. Cette méthode consiste à co-construire, sur la base de savoirs locaux et de connaissances des chercheurs, des jeux de rôles spécifiques permettant aux producteurs de simuler leur choix en termes de pratiques et d'en observer les effets en termes de production agricole, de revenu et de fourniture de services environnementaux. Lapplication de ces jeux de rôle dans deux zones de production de café au Costa Rica et au Nicaragua montre la diversité des réponses des producteurs face à des scénarios de mise en œuvre d'instruments de type coercitif (contrôle effectif de la législation environnementale) ou incitatif (crédit vert, certification et paiement pour services environnementaux). Bien que comportant certaines limites (méthode non prédictive), nous soulignons les intérêts de cette méthode pour concevoir des innovations en termes de gestion d'exploitation et d'instruments adaptés aux conditions des producteurs et à la diversité des systèmes agroforestiers.
\end{abstract}

Abstract. While the diversity of the environmental services provided by agroforestry systems are widely acknowledged, the nature and magnitude of the services they produce depends on producers' choices. Many policy instruments have been proposed to influence 
farmers' choice toward a higher provision of environmental services. However, anticipating the potential responses of producers to such instruments is a complex issue. In this chapter, we present the results of the application of an original methodology based on participatory and prospective approach conceived and implemented with local stakeholders and producers to test the interest of some instrument to promote environmental services provision. This method consists in co-designing thanks to local and scientific knowledge specific role-playing games that enable the producers to simulate their practices' choices and to observe their effects on their agricultural production, incomes and environmental services. The application of these role-playing games in two coffee producing areas in Costa Rica and Nicaragua, show the diversity of producers' responses according to scenarios of implementation of coercive (application of environmental legislation) and incentive measures (green credit, certification, payment for environmental services). Although this method has some limitation (no predictive results), we argue that it is of great interest to design innovations in terms of management and instruments adapted to farmers and SAF conditions.

\section{Introduction}

Le concept de services écosystémiques a été proposé pour caractériser les effets des écosystèmes sur la société (Millenium ecosystem assessment, 2005). Pour les agroécosystèmes, on distingue généralement les services d'approvisionnement (production agricole), de régulation (hydrique, séquestration de carbone, etc.), de support (conservation de la biodiversité et des sols) et les services culturels. À ces services, s'ajoutent les effets négatifs sur l'environnement (disservices - ayant un impact défavorable) tels que les pollutions ou l'érosion des sols qu'ils peuvent engendrer (Power, 2010; Swinton et al., 2007; Zhang et al., 2007).

Les systèmes agroforestiers sont reconnus pour l'importance et la multiplicité des services qu'ils fournissent (Davidson, 2005; Rapidel et al., 2011). Cependant, il est difficile de maximiser l'ensemble des services dans un même système agroforestier. Il est donc nécessaire de faire des compromis. Ces compromis entre différents services dépendent principalement des choix et des pratiques des agriculteurs. Souvent, ces derniers priorisent la production (les services d'approvisionnement) dont ils tirent un intérêt individuel ou familial au détriment d'autres services environnementaux d'intérêt général comme la protection de la qualité de l'eau ou la conservation de la biodiversité et des sols.

Afin d'encourager la production de services environnementaux, une large gamme d'instruments a déjà été proposée (Schroth et McNeely, 2011). Au-delà des instru-

\section{Encadré 4.1 - Services environnementaux et services écosystémiques}

Alors que les termes de services environnementaux et services écosystémiques sont parfois utilisés de manière synonyme dans la littérature, nous faisons ici la distinction entre ces deux concepts. À la différence des services écosystémiques, les services environnementaux n'incluent pas les services d'approvisionnement (production agricole). Ils représentent plus spécifiquement les services ayant un caractère de bien public (services de régulation ou de support) tels que la capture et le stockage du carbone affectant le changement climatique, les services hydrauliques (qualité et quantité d'eau), l'érosion des sols ou la biodiversité. 
ments de régulation publique fondés sur l'autorité (command and control) comme l'interdiction de certaines pratiques ou la mise en défens, les instruments économiques incitatifs font l'objet d'un intérêt croissant. Ces incitations économiques peuvent prendre différentes formes comme des paiements pour services environnementaux (Pirard et Lapeyre, 2014), des certifications socio-environnementales (Le Coq et al., 2016) ou des outils financiers de type microfinance verte (Huybrechs et al., 2016). Ces outils font souvent l'objet de débats (Le Coq et al., 2011), tant leur efficacité est variable en fonction des contextes dans lesquels ils sont élaborés et mis œuvre (Rodríguez-Robayo et Merino-Perez, 2017). Cette variabilité s'explique notamment par le manque de prise en compte des représentations et des stratégies des agriculteurs dans la conception de ces instruments. En effet, les approches dominantes pour la conception d'incitations économiques consistent à identifier les services fournis, évaluer leur valeur économique et en déduire le montant des incitations (Rapidel et al., 2011).

L'objectif de ce chapitre est de présenter les résultats de l'application d'une méthodologie originale fondée sur une approche à la fois participative et prospective conçue et mise en œuvre avec les acteurs locaux et les producteurs, afin de tester l'intérêt de certains instruments encourageant la fourniture de services environnementaux.

Après avoir explicité notre méthodologie, nous présenterons les résultats de son application dans deux régions d'étude au Costa Rica et au Nicaragua, dans le cadre du projet Safse (Compromis entre production et autres services écosytémiques fournis par les systèmes agroforestiers, Cirad/IRD) (Aucante, 2015; Bonifazi, 2015). Enfin, nous discuterons de l'intérêt et des limites de cette méthode et les perspectives qu'elle ouvre pour améliorer la définition d'instruments adaptés aux contextes agro-écologiques et socio-économiques des services agroforestiers.

\section{" Méthode de conception d'instruments par simulation participative}

La méthode mise en œuvre est inspirée des approches de modélisation d'accompagnement (ComMod) (Étienne, 2010) et mobilise le kit méthodologique Wat a game (Ferrand et al., 2009; De Fooij, 2011).

\section{Processus méthodologique des jeux de rôles}

Le processus méthodologique s'est déroulé en quatre étapes successives qui ont permis de co-construire des jeux de rôle et de réaliser des simulations participatives. Ces jeux de rôle ont été élaborés à partir d'un modèle générique original représentant les interactions possibles entre instruments, la décision des agriculteurs en termes d'activités, de choix de production agricole et de pratiques, ainsi que leurs effets en termes de fourniture de services environnementaux et de disservices au niveau de l'agroécosystème. Ce modèle générique a été conçu à partir de la littérature relative à l'analyse des systèmes de production (Cochet et Devienne, 2006) et des services écosystémiques dans les agroécosystèmes (Swinton et al., 2007; Zhang et al., 2007) (fig. 4.1). 


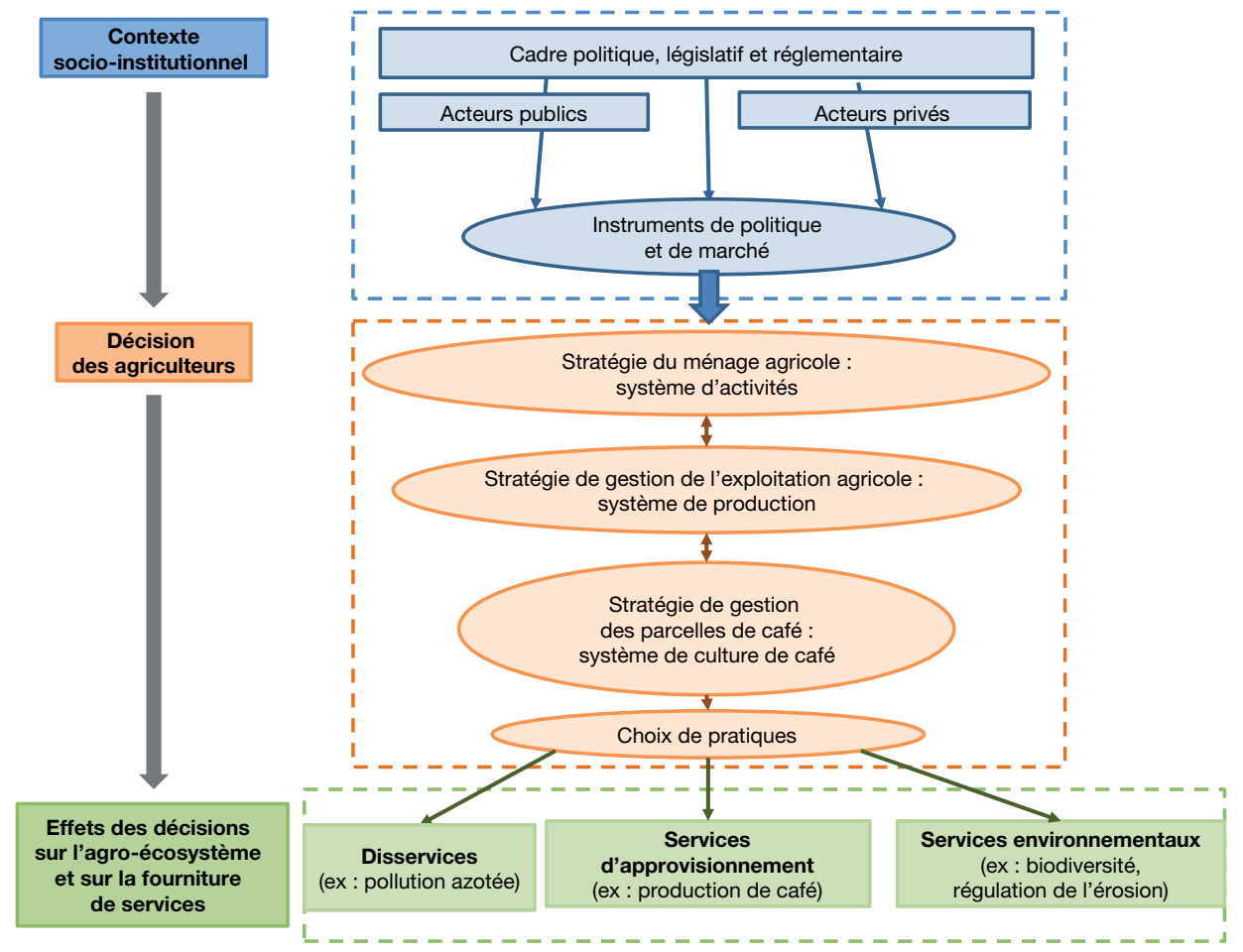

Figure 4.1. Cadre conceptuel pour appréhender les effets des instruments sur les pratiques des agriculteurs et la provision de services.

La première étape consiste en une analyse de l'agroécosystème, inspirée des approches de l'agriculture comparée (Cochet, 2011). Il s'agit d'abord d'identifier et de comprendre, grâce à des enquêtes auprès des agriculteurs, leurs pratiques agricoles et leur stratégie de gestion des systèmes agroforestiers en prenant en compte les contraintes et les opportunités liées à l'accès à des facteurs de production. En outre, en matière de contexte socio-institutionnel, les principaux instruments de politique et de marché existants (régulations, incitations, etc.), leur usage et leur représentation par les agriculteurs sont identifiés. En parallèle, une revue bibliographique et des entretiens d'experts locaux (conseillers de coopérative, gestionnaires de bassin versant, fonctionnaires, etc.) permettent d'analyser les relations entre la conduite des systèmes agroforestiers et leur fourniture de services écosystémiques.

La seconde étape a pour objectif de co-construire un jeu de rôle avec des experts locaux, des producteurs agricoles et des chercheurs pour tester de nouveaux instruments dans un environnement représentant l'agroécosystème de leur territoire. Pour ce faire, nous avons mobilisé le kit méthodologique Wat a game. Cette méthodologie, initialement créée pour la gestion de l'eau, permet la co-construction d'un modèle à dire d'acteurs afin d'aborder des problématiques de gestion de ressources. Impliquant des acteurs locaux (dont les producteurs, futurs joueurs des simulations) et des chercheurs porteurs de savoirs scientifiques, elle permet de construction selon une démarche en jouant (design by playing) des itérations de dialogues afin d'élaborer un modèle (symbolisé sous forme de jeu) représen- 
tant les socio-écosystèmes locaux. Ainsi, dans notre application de cette méthode, les objectifs des jeux et leurs structures, ainsi que les règles du jeu (scenarios en termes de mise en place d'instruments) ont été déterminés lors d'une série d'ateliers avec les experts locaux et chercheurs du projet Safse. Ces ateliers ont aussi permis un calibrage des pratiques et des activités (cartes «actions »), c'est-à-dire la définition des ressources (moyens de production) nécessaires à la mise en œuvre de chaque pratique et activité (intrants), ainsi que leurs résultats respectifs (en termes de fourniture de services et de revenu). Les liens entre pratiques et fourniture de services sont ainsi définis par croisement de savoirs locaux et savoirs des experts, qui s'appuient sur des recherches conduites dans d'autres situations et, évidemment, d'autres dispositifs expérimentaux spécifiques (Villatoro et al., 2015; Meylan et al., 2017; voir également chapitres xx).

Dans une troisième phase, des sessions de jeu d'environ trois heures sont organisées. Les agriculteurs sont invités à «jouer» leur propre rôle, c'est-à-dire à choisir des pratiques agricoles et des activités dans leurs exploitations stylisées sur un plateau de jeu. Après une présentation générale, ils reçoivent des ressources (intrants) symbolisés par des perles de couleurs différentes. Ces ressources leur permettent à chaque tour de mettre en œuvre des activités sur leur exploitation. À la fin d'un tour représentant une année, les cartes «actions» produisent des résultats (outputs). Chaque joueur évalue alors ses résultats et la fourniture de services écosystémiques de son exploitation. Finalement, une évaluation collective (somme des résultats individuels) est faite à l'échelle du territoire symbolisé par un plateau central. À chaque tour de jeu, un nouveau scénario en termes de mise en place d'instruments est introduit par l'animateur. Une fois les différents scénarios simulés, les joueurs commentent leur choix pendant le jeu et à donner leur avis sur les instruments proposés.

La dernière phase consiste à analyser les résultats des sessions de jeu. Cette analyse porte d'une part sur des comportements des agriculteurs en termes d'adoption de pratiques et d'activités, et d'autre part sur les résultats collectifs en termes de fourniture de services écosystémiques selon les scenarios. Pour ce faire, les résultats des tours, où des instruments ont été testés, sont comparés à ceux de la situation de référence correspondant à un premier tour de jeu sans incitations.

\section{Application dans deux régions caféières au Costa Rica et au Nicaragua}

La méthode décrite précédemment a été mise en œuvre dans le canton de Llano Bonito au Costa Rica et dans la commune d'El Tuma-La Dalia au Nicaragua. Quoique différents en termes agroécologique, socio-économique et de contexte institutionnel (tab. 4.1), ces deux territoires sont des zones de production de café d'altitude, avec une forte présence de systèmes agroforestiers (voir chapitres 1 et 15).

Suivant des principes généraux similaires, deux jeux ont été conçus afin de rendre compte des spécificités des deux régions en termes de moyens de production, de pratiques et de services écosystémiques.

Par ailleurs, les pratiques et activités que les agriculteurs peuvent choisir diffèrent entre les deux sites (tab. 4.2 et tab. 4.3). 
Tableau 4.1. Caractéristiques agroécologiques et socio-économiques des territoires d'étude au Costa Rica et au Nicaragua.

\begin{tabular}{lcc}
\hline \multicolumn{1}{c}{ Caractéristiques } & Llano Bonito (Costa Rica) & La Dalia (Nicaragua) \\
\hline Altitude $(\mathrm{m})$ & $1200-1900$ & $700-1200$ \\
\hline Précipitation moyenne annuelle $(\mathrm{mm})$ & 2440 & 1890 \\
\hline Productivité en café $(\mathrm{kg} / \mathrm{ha})$ & 1440 (Warren Raffa, 2013) & 720 (Notaro, 2014) \\
\hline Densité de la population (hab./km²) & 62 (Inec) & 139 (Inifom) \\
\hline Indice de développement humain ${ }^{(1)}$ & $0,69(2016)$ & $0,45(2012)$ \\
\hline
\end{tabular}

(1) Lindice de dévelopement humain est un indice composite calculé par le PNUD, compris entre 0 (très mauvais) et 1 (excellent). Il est calculé à partir de la moyenne de trois indices quantifiants : la santé et la longévité, le savoir et le niveau d'éducation, ainsi que le niveau de vie. Source pour Llano Bonito, Costa Rica : canton de Leon Cortes (http://desarrollohumano.or.cr/mapa-cantonal/index.php/rankingidh\#ranking-por-idh, accedé le 29/03/2019). Source pour La Dalia Nicaragua, http://hdr.undp.org/sites/ default/files/nicaragua_2002_sp.pdf, accedé le 29/03/2019.

\section{Au Costa Rica}

Les paramètres du jeu. Au Costa Rica, deux ressources (inputs) ont été retenus : l'argent et la main-d'œuvre. En effet, au-delà de la contrainte monétaire, les agriculteurs font face à une pénurie de main-d'œuvre les contraignant à employer des salariés lors des pics de travaux. En termes de résultats (outputs), en plus de la production de café et de la conservation de la biodiversité, sont pris en compte l'érosion des sols et la qualité des eaux. En effet, le bassin versant fait face à de graves problèmes d'érosion des sols menaçant le fonctionnement d'un barrage en aval et à des pollutions des eaux (eutrophisation des cours d'eau) liées à un usage massif d'engrais azotés. Quant à la biodiversité, elle est au cour de politiques nationales et est une préoccupation pour les acteurs locaux.

Les cartes actions permettent de simuler les choix en termes de gestion de la parcelle de caféier (lutte contre les adventices, fertilisation et type d'ombrage), d'aménagement des parcelles (terrasse, mise en défens d'une bande non cultivée le long des cours d'eau) et d'activités complémentaires (agricoles ou non agricoles).

Les scénarios. En termes d'instruments, trois scénarios sont simulés pendant les sessions de jeux.

Scénario 1. Mise en place d'un contrôle de l'interdiction de produire sur une bande proche des cours d'eau (protection des rivières). Cela diminue la surface de production, et donc la production de café, et réduit les risques d'érosion et de pollution azotée des cours d'eau. Cette interdiction est inscrite dans la loi du Costa Rica, mais de fait peu contrôlée par les autorités. Or, les limites de ce type de mesure (de type «command and control») sont souvent soulignées pour justifier le recours à des instruments incitatifs du type paiements pour services environnementaux (Wunder, 2005), alors qu'elles peuvent être efficaces si le contrôle est effectif (Börner et al., 2014).

Scénario 2. Mise en place de «crédits verts», c'est-à-dire un financement à taux zéro pour l'adoption de pratiques permettant de réduire les disservices, demandant des investissements financiers non accessibles aux agriculteurs. Ces crédits sont 


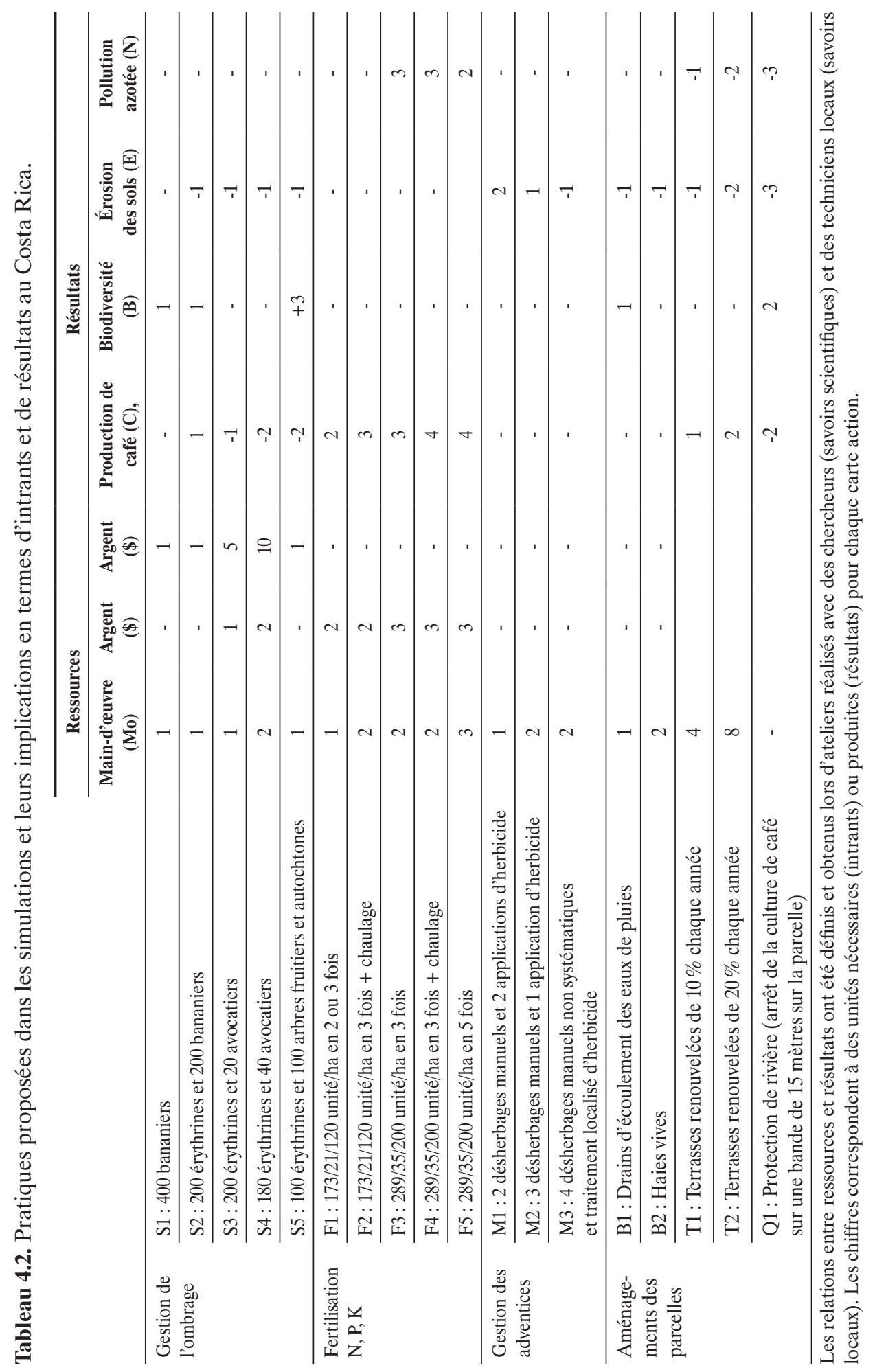


proposés pour diminuer et fractionner les apports de fertilisants (F1 et F2); ce qui peut affecter la production, mais diminue les risques de pollution azotée. Un crédit est aussi possible pour financer le désherbage manuellement (à la place du traitement herbicide) et l'aménagement des terrasses, deux pratiques limitant l'érosion et coûteuses en main-d'œuvre (M3). Ce type d'instrument n'existe pas pour l'instant au Costa Rica, mais il s'apparente au programme de reconnaissance pour bénéfices environnementaux mis en place par le ministère de l'Agriculture depuis 2007 pour faciliter l'adoption de pratiques agricoles soucieuses de protéger l'environnement (Bonin et al., 2017). Comme l'idée de la mise en place d'un crédit vert se diffuse dans d'autres pays pour favoriser la provision de services écosystémiques (Huybrechs et al., 2016), il semble intéressant de tester ce type d'instrument.

Scénario 3. Mise en place de paiements pour services environnementaux sous forme d'une compensation monétaire pour le manque à gagner du fait du développement d'un ombrage diversifié (S5) ou de la protection des rivières par des bandes enherbées (Q1). Ces deux pratiques réduisent la production de café. Néanmoins, elles augmentent les services de régulation ou de support (biodiversité) et réduisent les disservices (érosion des sols et pollution azotée). Cet instrument a été choisi car, même s'il existe un programme national de paiements pour services environnementaux au Costa Rica, ce programme est peu mobilisé par les agriculteurs dans la zone parce qu'il est peu connu et peu adapté à leur réalité. Il s'agit donc de tester les effets potentiels d'une modalité d'un paiement pour service environnemental nouveau avec des conditionnalités plus adaptées à la situation de ces agriculteurs de systèmes agroforestiers à base de caféiers.

\section{Au Nicaragua}

Les paramètres du jeu. Au Nicaragua, la principale ressource limitante est la disponibilité monétaire liée à la pauvreté rurale. Par conséquent, c'est le seul intrant représenté dans le jeu. Pour les résultats attendus, les services d'approvisionnement considérés sont la production de café et la production de cultures vivrières (maïs) pour l'alimentation familiale. En effet, la sécurité alimentaire est un enjeu fondamental pour les ménages ruraux de cette région isolée et pauvre. En outre, deux disservices sont représentés à cause de leur importance sur la qualité de l'eau de consommation en aval de la zone : la pollution organique liée au dépulpage du café dont les effluents sont rejetés dans les rivières, et la pollution chimique liée à l'usage de produits phytosanitaires.

Les cartes actions ont permis de simuler des choix en termes de systèmes de culture des caféiers (quatre modes de gestion allant de systèmes agroforestiers avec fort ombrage à des systèmes de culture de caféiers de plein soleil), et des pratiques culturales spécifiques liées à l'application d'intrants agrochimiques (fertilisation et produits phytosanitaires), la diversification des cultures, le traitement des effluents de transformation du café, la lutte contre les bioagresseurs et les aménagements de la parcelle.

Au Nicaragua, les trois scénarios étaient les suivants.

Scénario 1 : application de sanctions (renforcement de la mise en place du cadre législatif existant) et de crédit de campagne (pour lever la contrainte financière des 
Tableau 4.3. Pratiques proposées dans les simulations et leurs implications en termes d'intrants et de résultats au Nicaragua.

\begin{tabular}{|c|c|c|c|c|c|c|}
\hline & & \multicolumn{2}{|c|}{ Ressources } & \multicolumn{3}{|c|}{ Résultats } \\
\hline & & $\begin{array}{l}\text { Argent } \\
(\$)\end{array}$ & $\begin{array}{l}\text { Production } \\
\text { de café }(C)\end{array}$ & $\begin{array}{l}\text { Production } \\
\text { alimentaire } \\
\text { (A) }\end{array}$ & $\begin{array}{l}\text { Pollution } \\
\text { chimique } \\
\quad(\mathrm{PC})\end{array}$ & $\begin{array}{c}\text { Pollution } \\
\text { organique } \\
\text { (PO) }\end{array}$ \\
\hline \multirow{4}{*}{$\begin{array}{l}\text { Mode de } \\
\text { gestion de } \\
\text { la parcelle de } \\
\text { café : } 4 \text { niveaux } \\
\text { d'intensification }\end{array}$} & Café «naturel» & 9 & 10 & 6 & 5 & 9 \\
\hline & Café «simple» & 28 & 15 & 2 & 10 & 28 \\
\hline & Café «semi-technifié» & 45 & 30 & 0 & 25 & 45 \\
\hline & Café «technifié» & 77 & 80 & 0 & 50 & 77 \\
\hline \multirow{4}{*}{$\begin{array}{l}\text { Intensification : } \\
\text { application } \\
\text { d'intrants } \\
\text { chimiques, } \\
\text { fertilisants et } \\
\text { phytosanitaires }\end{array}$} & $\begin{array}{l}\text { P1 : } 1 \text { application NPK / } \\
1 \text { application de phytosanitaires }\end{array}$ & 6 & $+10 \%$ & $+10 \%$ & +2 & \\
\hline & $\begin{array}{l}\text { P2 }: 2 \text { applications NPK/ } \\
2 \text { applications de phytosanitaires }\end{array}$ & 12 & $+20 \%$ & $+20 \%$ & +5 & \\
\hline & $\begin{array}{l}\text { P3 : } 2 \text { applications NPK/ } \\
4 \text { applications de phytosanitaires }\end{array}$ & 15 & $+30 \%$ & $+30 \%$ & +10 & \\
\hline & $\begin{array}{l}\mathrm{P} 4: 3 \text { applications NPK/ } \\
6 \text { applications de phytosanitaires }\end{array}$ & 22 & $+40 \%$ & $+40 \%$ & +15 & \\
\hline \multirow{3}{*}{$\begin{array}{l}\text { Diversification } \\
\text { culturale }\end{array}$} & Association avec du cacaoyer & 12 & 50 & & 5 & \\
\hline & Association avec des bananiers & 2 & -3 & 10 & & \\
\hline & Association avec des fruitiers & 3 & -3 & 15 & & \\
\hline \multirow{5}{*}{$\begin{array}{l}\text { Aménagement } \\
\text { parcellaire }\end{array}$} & Barrière morte & 2 & & & -2 & \\
\hline & Barrière vive & 3 & & & -4 & \\
\hline & Couverture du sol & 2 & & & -3 & \\
\hline & Parcelle forestière & 5 & & & -2 & \\
\hline & $\begin{array}{l}\text { Éloignement des cultures } \\
\text { du cours d'eau }\end{array}$ & 1 & & & -1 & \\
\hline \multirow{2}{*}{$\begin{array}{l}\text { Lutte contre les } \\
\text { bioagresseurs }\end{array}$} & Pièges à scolyte & 0,5 & & & -2 & \\
\hline & $\begin{array}{l}\text { Tri des grains touchés } \\
\text { par le scolyte }\end{array}$ & 1 & & & -2 & \\
\hline \multirow{4}{*}{$\begin{array}{l}\text { Pratiques } \\
\text { alternatives } \\
\text { à l'utilisation } \\
\text { d'intrants } \\
\text { chimiques }\end{array}$} & $\begin{array}{l}\text { Rotation des fongicides } \\
\text { (molécules) }\end{array}$ & 0,5 & & & -1 & \\
\hline & $\begin{array}{l}\text { Usage « raisonné» } \\
\text { des herbicides }\end{array}$ & 3 & & & -2 & \\
\hline & Fertilisation « raisonnée» & 0 & & & -2 & \\
\hline & Amendements organiques & 3 & & & -4 & \\
\hline \multirow{5}{*}{$\begin{array}{l}\text { Gestion des } \\
\text { effluents et } \\
\text { sous-produits } \\
\text { du café }\end{array}$} & Dépulpeuse en circuit fermé & 28 & & & & $-10 \%$ \\
\hline & Trou avec des pierres & 2 & & & & $-20 \%$ \\
\hline & Fosse ou réservoir d'oxydation & 2 & & & & $-50 \%$ \\
\hline & Lagune avec système de filtres & 14 & & & & $-80 \%$ \\
\hline & Démucilagineuse & 30 & & & & $-100 \%$ \\
\hline
\end{tabular}

Les relations entre les ressources et les résultats ont été définis lors d'ateliers réalisés avec des chercheurs (savoirs scientifiques) et des techniciens locaux (savoirs locaux). Les chiffres correspondent à des unités nécessaires (inputs) ou produites (outputs) pour chaque carte «actions». 
exploitations). En effet, dans ce territoire où la contrainte d'accès au crédit est forte, il s'agit de voir si en levant cette contrainte financière, qui limite la production du café, et en faisant appliquer effectivement les régulations actuelles, les producteurs se tournent vers des pratiques plus respectueuses de l'environnement.

Scénario 2 : mise en place de «crédits verts » basés sur les remises d'intérêts conditionnés par l'adoption de certaines pratiques plus respectueuses de l'environnement, parallèlement à la fourniture d'assistance technique permettant de faciliter l'adoption de ces pratiques.

Scénario 3 : mise en place d'une certification du café de type commerce équitable, mais avec des conditionnalités sur l'adoption de pratiques respectueuses de l'environnement. Cette certification offre une garantie sur les prix du café tout en obligeant à adopter certaines pratiques. Ce type d'instruments existe au Nicaragua, mais il est encore peu développé dans cette zone et surtout, ne correspond pas aux contraintes des petits producteurs qui sont majoritaires.

\section{Résultats des simulations participatives}

Quatre sessions de jeux avec treize agriculteurs au total sont réalisées au Costa Rica et trois sessions avec quinze joueurs au total au Nicaragua. Ces simulations participatives mettent en lumière des réponses différentes selon les scenarios en termes d'adoption de pratiques et de provision de services écosystémiques.

\section{Au Costa Rica}

Les résultats sont présentés dans le tableau 4.4. La mise en place d'un paiement pour services environnementaux ciblé sur le multimultiombrage (S5) incluant des arbres d'espèces autochtones incite bien l'adoption de cette pratique actuellement peu mise en œuvre. La gestion des adventices basée sur un faible usage d'intrants chimiques et des désherbages manuels (M3) est également davantage adoptée avec la mise en place d'incitation positive de type «crédit vert» ou de paiements pour services environnementaux. La fertilisation réduite, raisonnée et fractionnée (F1) est peu adoptée quels que soient les scenarios. Seule la fertilisation réduite (F2) est adoptée de manière accrue avec les scénarios de «crédit vert» ou de paiements pour services environnementaux. Enfin, la mise en place d'une zone de protection proche du cours d'eau (Q1) semble être favorisée par la mise en place d'un contrôle de l'interdiction de produire sur une bande proche des cours d'eau (protection des rivières) et par la mise en place d'un paiement pour services environnementaux spécifique. L'effet de ce paiement semble légèrement supérieur à celui d'un contrôle accru.

En vertu des relations entre pratiques et provision de services écosystémiques définis pour chacune des pratiques (tab. 4.2), les choix de pratiques réalisés dans le jeu selon les scenarios entraînent une fourniture plus ou moins importante des différents services écosystémiques (tab. 4.5). Le renforcement du contrôle des régulations existantes semble ainsi réduire les disservices, mais il conduit aussi à une réduction de la production de café. La mise en place de crédits verts permet d'encourager la diversification des pratiques (la fertilisation raisonnée F1 et F2 et le désherbage manuel M3) favorables à la réduction des disservices (érosion du sol et pollution 
azotée), tout en conduisant à une réduction pluslimitée de la production de café. Enfin, la mise en place de paiements pour services environnementaux en promouvant la gestion multiombrage (S5) et la mise en place d'une zone de protection des rivières $(\mathrm{Q} 1)$ conduisent à une augmentation des services écosystémiques de support (biodiversité) et une réduction des disservices (érosion et pollution). Cependant, cela se fait au détriment de la production de café qui est fortement réduite.

\section{Au Nicaragua}

Les simulations montrent aussi dans le contexte du Nicaragua des résultats différents selon les scénarios en termes d'adoption de pratiques selon les scénarios

Tableau 4.4. Effets simulés des scenarios selon les types d'instruments sur l'adoption de pratiques de systèmes agroforestiers et les aménagements des parcelles dans la zone d'étude au Costa Rica.

\begin{tabular}{|c|c|c|c|c|c|c|c|c|c|c|c|c|c|c|c|c|c|c|}
\hline \multirow[t]{2}{*}{ Scenarios } & \multicolumn{5}{|c|}{$\begin{array}{c}\text { Gestion } \\
\text { de l'ombrage }\end{array}$} & \multicolumn{5}{|c|}{$\begin{array}{l}\text { Fertilisation } \\
\text { des caféiers }\end{array}$} & \multicolumn{3}{|c|}{$\begin{array}{l}\text { Gestion des } \\
\text { adventices }\end{array}$} & \multicolumn{5}{|c|}{$\begin{array}{c}\text { Aménagement } \\
\text { des parcelles }\end{array}$} \\
\hline & $\mathrm{S} 1^{(1)}$ & S2 & $\mathrm{S} 3$ & S4 & S5 & $\mathrm{F} 1$ & $\mathrm{~F} 2$ & F3 & $\mathrm{F} 4$ & F5 & M1 & M2 & M3 & B1 & B2 & $\mathrm{T} 1$ & $\mathrm{~T} 2$ & Q1 \\
\hline $\begin{array}{l}\text { Situation } \\
\text { de référence }\end{array}$ & 1 & 5 & 2 & 4 & 1 & 0 & 1 & 6 & 5 & 1 & 0 & 12 & 1 & 9 & 1 & 6 & 5 & 6 \\
\hline $\begin{array}{l}\text { Contrôle effectif } \\
\text { de la protection } \\
\text { des rivières }\end{array}$ & 0 & 3 & 0 & 5 & 5 & 0 & 1 & 4 & 6 & 2 & 1 & 8 & 4 & 6 & 3 & 6 & 5 & 10 \\
\hline Crédit vert & 1 & 4 & 3 & 1 & 4 & 1 & 6 & 2 & 4 & 0 & 0 & 5 & 8 & 6 & 1 & 5 & 8 & 8 \\
\hline
\end{tabular}

Note. Les nombres correspondent au nombre de pratiques adoptées par les agriculteurs dans les sessions de jeux pour chaque type de pratiques, sur un total de 13 joueurs ayant participé aux simulations. Pour les pratiques de gestion d'ombrage, de fertilisation et de gestion des adventices, un seul choix était possible. Pour l'aménagement des parcelles, les agriculteurs pouvaient en choisir plusieurs.

(1) Les codes correspondent aux pratiques présentées dans le tableau 4.2.

(2) La situation de référence correspond au premier tour du jeu pour laquelle les pratiques sont les plus courantes dans la réalité.

Tableau 4.5. Résultats des simulations de scénario de type d'instrument en termes de production de services et disservices produits à l'échelle du bassin versant.

\begin{tabular}{lcccc}
\hline \multicolumn{1}{c}{ Scenarios } & \multicolumn{4}{c}{$\begin{array}{c}\text { Évolution des services en comparaison } \\
\text { de la situation de référence }\end{array}$} \\
\cline { 2 - 5 } & Café & Érosion & Biodiversité & Pollution \\
\cline { 2 - 5 } & -- & - & ++ & - \\
\hline $\begin{array}{l}\text { Contrôle effectif } \\
\text { de la protection des rivières }\end{array}$ & - & -- & + & -- \\
\hline Crédit vert & -- & -- & ++ & -- \\
\hline $\begin{array}{l}\text { Paiement pour services } \\
\text { environnementaux }\end{array}$ & - & & & \\
\hline
\end{tabular}

Lintensité des changements est indiquée de manière qualitative par le nombre de signes :

+ signifie une légère augmentation du nombre d'unité de service fournit dans le scénario en question vis-à-vis de la situation de référence, ++ une augmentation modérée, et +++ une forte augmentation; - signifie une légère diminution du nombre d'unité de service fournit dans le cas du scénario en question vis-à-vis de la situation de référence, -- une réduction modérée, et --- une forte réduction 
(tab. 4.6). Le renforcement des contrôles, couplé à un meilleur accès au crédit, est le scénario qui conduit à davantage d'adoption de pratiques limitant la pollution chimique et, dans une moindre mesure, à des pratiques raisonnées d'application d'intrants chimiques. Ceci confirme l'efficience de la mise en place effective d'un contrôle accru dans le contexte du jeu. En revanche, ce sont les mesures incitatives qui conduisent à traiter davantage les effluents de transformation du café qui entraînent une pollution organique. Si ce résultat confirme l'importance du manque de moyens financiers pour l'adoption de pratiques plus respectueuses pour l'environnement, on constate que les incitations fondées sur la stabilité des prix (certification du café) semblent légèrement plus efficaces que des crédits ciblés (crédit vert). Enfin, c'est avec le scénario des «crédits verts» que les pratiques alternatives de lutte contre les bioagresseurs réduisant les pollutions chimiques sont le plus adoptées.

Tableau 4.6. Effets simulés des scenarios de changement d'environnement institutionnel sur l'adoption de pratiques dans la zone d'étude au Nicaragua.

\begin{tabular}{|c|c|c|c|c|c|}
\hline Scénarios & $\begin{array}{c}\text { Aménagement } \\
\text { parcellaires }\end{array}$ & $\begin{array}{l}\text { Pratiques de } \\
\text { diversification } \\
\text { culturale }\end{array}$ & $\begin{array}{c}\text { Gestion des } \\
\text { effluents et } \\
\text { sous-produits } \\
\text { du café }\end{array}$ & $\begin{array}{c}\text { Intensification } \\
\text { (pratiques } \\
\text { en matière } \\
\text { d'application } \\
\text { d'intrants } \\
\text { chimiques) }\end{array}$ & $\begin{array}{c}\text { Pratiques } \\
\text { culturales } \\
\text { alternatives et } \\
\text { de lutte contre } \\
\text { les bioagresseurs } \\
\text { réduisant } \\
\text { la pollution } \\
\text { chimique }\end{array}$ \\
\hline $\begin{array}{l}\text { Corps de règles } \\
\text { en vigueur et } \\
\text { sanctions + } \\
\text { crédit classique }\end{array}$ & 18 & 7 & 8 & 4 & n.d. \\
\hline $\begin{array}{l}\text { «Crédit vert» } \\
\text { et formation } \\
\text { technique }\end{array}$ & 15 & 3 & 13 & 1 & 44 \\
\hline $\begin{array}{l}\text { Certification } \\
\text { commerce } \\
\text { équitable }\end{array}$ & 14 & 7 & 15 & 0 & 25 \\
\hline
\end{tabular}

Les chiffres indiquent le nombre total de pratiques adoptées par les agriculteurs (15 au total) lors des sessions de simulation participatives par types de pratiques. Un producteur peut adopter plusieurs pratiques.

n.d. Données non disponibles, les pratiques alternatives réduisant la pollution chimique n'ayant été introduite que pour les scénarios de crédit vert et de certification.

En termes de services et disservices, les simulations montrent que les trois scenarios proposés conduisent in fine à des situations assez similaires en termes de réduction des pollutions chimiques et organiques et d'augmentation de la production alimentaire (tab. 4.7). Toutefois, ces améliorations en termes de réduction des impacts environnementaux s'accompagnent d'une réduction de la production de café dans le cas des incitations positives (crédit vert + assistance technique et certification). Dans le cas d'un renforcement des contrôles des règles existantes (couplé à un accès au crédit de campagne), ces changements s'accompagnent d'une très légère augmentation de la production de café. Cette augmentation peut s'expliquer par le caractère aléatoire des contrôles simulés dans le jeu. En effet, les joueurs contrôlés 
ont subi une certaine pression les incitant à utiliser leur crédit de campagne pour implémenter des pratiques ayant des effets positifs sur les services environnementaux. Certains joueurs non soumis au contrôle et bénéficiant du crédit de campagne ont saisi cette opportunité pour appliquer davantage d'intrants chimiques et ainsi augmenter leur production de café.

Tableau 4.7. Résultats des simulations de scénario de changement institutionnel en termes de services et disservices dans la zone d'étude du Nicaragua.

\begin{tabular}{lcccc}
\hline \multicolumn{1}{c}{ Scénarios } & \multicolumn{4}{c}{ Évolution en comparaison du scenario de référence } \\
\cline { 2 - 5 } & $\begin{array}{c}\text { Production } \\
\text { de café }\end{array}$ & $\begin{array}{c}\text { Production } \\
\text { d'aliments }\end{array}$ & $\begin{array}{c}\text { Pollution } \\
\text { chimique }\end{array}$ & $\begin{array}{c}\text { Pollution } \\
\text { organique }\end{array}$ \\
\hline $\begin{array}{l}\text { Corps de règles en vigueur } \\
\text { et sanctions + crédit classique }\end{array}$ & + & +++ & -- & -- \\
\hline «Crédit vert» & - & ++ & --- & -- \\
et formation technique & - & ++ & ---- & -- \\
\hline Certification commerce équitable & - & & & \\
\hline
\end{tabular}

Lintensité des changements est indiquée de manière qualitative par le nombre de signes :

,,++++++ : intensité de l'augmentation des unités de services considérée par rapport au tour de référence;

-, --, --- : intensité de la diminution des unités de la ressource considérée par rapport au tour de référence.

\section{Limites et perspectives la méthode}

La mise en œuvre de ces jeux, en simplifiant la réalité et en élargissant le domaine des possibles, permet de générer une réflexion au-delà d'une simple vision tendancielle en introduisant l'idée de rupture (les scenarios). Les différentes sessions peuvent ainsi faire émerger de nouvelles idées d'actions. Par exemple au Nicaragua, dans le jeu, les acteurs recherchent des solutions collectives pour faire face aux pollutions. Cette démarche était relativement nouvelle dans ce site sur ce thème. Les participants soulignent aussi l'intérêt de cet outil pour prendre conscience des enjeux environnementaux, du lien entre choix individuels et résultats collectifs. Loutil permet aussi de réfléchir à la mise en œuvre de pratiques innovantes, auxquelles les joueurs n'avaient pas forcément pensé. Plus généralement, les joueurs s'accordent à dire que le jeu leur a semblé être une méthode attractive et compréhensible pour échanger.

La méthode comporte néanmoins des limites. En effet, le jeu n'est pas prédictif, comme peuvent le prétendre des modèles et simulations calibrés sur la base de faits qui se répètent et pour lesquels des données quantitatives sont disponibles. Au contraire, il s'agit d'une approche prospective pour envisager des futurs possibles et en explorer les conséquences possibles par rapport à un futur désiré. Des améliorations peuvent être apportées à la méthode présentée afin de gagner en robustesse. La première amélioration concerne l'intégration d'une connaissance plus fine des fonctionnements des agroécosystèmes, et des coûts et bénéfices des pratiques en termes monétaire et de provision de services. Le calibrage du jeu serait ainsi plus robuste. La seconde amélioration concerne le nombre de répétitions des sessions de jeu. Dans nos applications, nous avons pu mettre en place un nombre limité de répé- 
titions de sessions de jeu (7 sessions de jeu, 28 producteurs au total). Un nombre plus important doit permettre d'avoir une meilleure robustesse statistique sur les réponses des agriculteurs face aux scénarios proposés.

Malgré ces limites, la méthode proposée offre des perspectives pour concevoir des innovations en termes de gestion d'exploitation agricole et d'instruments dans le cadre de projets de recherche ou de développement. Elle pourrait notamment être adaptée pour tester une plus grande diversité d'instruments politiques et législatifs concernant les services environnementaux, une gamme réduite d'instruments en raffinant les conditionnalités et les modalités (montant d'un paiement pour services environnementaux, conditionnalité spécifique) ou encore une combinaison d'instruments (assistance technique, financement, etc.) Cette méthode pourrait aussi être mobilisée pour identifier des objectifs communs à l'échelle du territoire et renforcer l'émergence d'actions collectives. Pour ce faire, plusieurs évolutions du jeu sont envisageables, comme demander aux agriculteurs de proposer eux-mêmes des options de scénarios et/ou inclure des rôles supplémentaires correspondant aux acteurs institutionnels existants (agence de gestion du bassin et coopérative). Enfin, ce type de simulation pourrait être inséré dans des processus de renforcement de capacités en gestion des exploitations, dans la mesure où il permet de tester virtuellement des combinaisons d'activités économiques et des pratiques agricoles.

\section{" Bibliographie}

Abrami G., Ferrand N., Morardet S., Murgue C., Popova A., De Fooij H., Farolfi S., Dutoit D., Aquae-Gaudi W. 2012. Wat-A-Game, a toolkit for building role-playing games about integrated water management. In: Managing resources of a limited planet: pathways and visions under uncertainty, sixth biennial meeting. Seppelt R., Voinov A.A., Lange S., Bankamp D. (eds). International congress on environmental modelling and software (IEMSS). 6, Leipzig, Allemagne, 01/06/201205/07/2012. Leipzig: IEMSS, 1912-1919. ISBN 978-88-9035-742-8.

Aucante M., 2015. Arrangements institutionnels et fourniture de services écosystémiques dans un territoire agroforestier. Construction et application d'un outil de jeu de rôles. Master, Istom.

Bonifazi M., 2015. Services écosystémiques et production agroforestière : instruments d'incitation pour le bassin de Llano Bonito, Costa Rica. Master, IRC-SupAgro Montpellier.

Bonin M., Le Coq J.F., Aznar O., Saenz F., 2017. Efficacité et justice des instruments de politiques agricoles visant à favoriser la provision de services environnementaux : une analyse comparée entre Costa Rica et France (Guadeloupe). VertigO, 17(2): 19 p.

Börner J., Wunder S., Wertz-Kanounnikoff S., Hyman G., Nascimento N., 2014. Forest law enforcement in the brazilian amazon: Costs and income effects. Global environmental change, 29 : 294-305.

Étienne M. (ed.), 2010. La modélisation d'accompagnement. Une démarche participative en appui au développement durable. Versailles, France : Éditions Quæ, 384 p.

Cochet H., Devienne S., 2006. Fonctionnement et performances économiques des systèmes de production agricole : une démarche à l'échelle régionale. Cahiers agricultures, 15(6) : 578-583.

Davidson S., 2005. Shade coffee agro-ecosystems in Mexico: a synopsis of the environmental services and socio-economic considerations. Journal of sustainable forestry, 21(1): 81-95.

De Fooij H., 2011. Wat-a-game: modelling a participatory role-playing game. Mémoire de fin d'études, Université de Twente, Pays Bas.

Ferrand N., Farolfi S., Abrami G., Dutoit D., 2009. Wat-a-game: sharing water and policies in your own basin, In: $40^{\text {th }}$ Annual Conference. International simulation and gaming association, Singapore, 17 p. https://hal.archives-ouvertes.fr/hal-01355501/document (consulté le 07/04/2019). 
Huybrechs F., Bastiaensen J., Forcella D., Van Hecken G., 2016. La microfinance pour les services environnementaux. Revue tiers monde, $1: 125-154$.

Le Coq J.F., Serpantié G., Andriamahefazafy F., Saenz-Segura F., 2016. Les écocertifications, des dispositifs en faveur des services écosystémiques? In : Méral P. et Pesche D., (eds). Les services écosystémiques : repenser les relations nature et société. . Versailles, France : Éditions Quae, 213-228.

Le Coq J.F., Soto G., González Hernández C., 2011. PES and eco-label. A comparative analysis of their limits and opportunities to foster environmental services provision. In: Rapidel B., De Clerk F., Le Coq J.F., Beer J., (eds). Ecosystem services from agriculture and agroforestry: measurement and payment. Londres, Royaume-Uni: Earthscan publications, 237-264.

Millenium ecosystem assessment, 2005. Ecosystems and human well-being. Washington DC : Island Press.

Méral P., Pesche D., 2016. Les services écosystémiques : repenser les relations nature et société. Versailles, France : Éditions Quae, 300 p.

Meylan L., Gary C., Allinne C., Ortiz J., Jackson L., Rapidel B., 2017. Evaluating the effect of shade trees on provision of ecosystem services in intensively managed coffee plantations. Agriculture, ecosystems and environment, 245: 32-42.

Pirard R., Lapeyre R., 2014. Classifying market-based instruments for ecosystem services: a guide to the literature jungle. Ecosystem services, 9: 106-114.

Power A.G., 2010. Ecosystem services and agriculture: tradeoffs and synergies. Philosophical transactions of the royal society B: biological sciences, 365(1554): 2959-2971.

Rapidel B., De Clerck F., Le Coq J.F., Beer J., (eds), 2011. Ecosystem services from agriculture and agroforestry: measurement and payment. Londres, Royaume-Uni : Earthscan publications.

Rodríguez-Robayo K.J., Merino-Perez L., 2017. Contextualizing context in the analysis of payment for ecosystem services. Ecosystem services, 23: 259-267.

Schroth G., McNeely J.A., 2011. Biodiversity conservation, ecosystem services and livelihoods in tropical landscapes: towards a common agenda. Environmental management, 48(2): 229-236.

Swinton S.M., Lupi F., Robertson G.P., Hamilton S.K., 2007. Ecosystem services and agriculture: cultivating agricultural ecosystems for diverse benefits. Ecological economics, 64(2): 245-252.

Villatoro Sanchez M., Wang Y., Stokes A., 2017. Vegetation as a driver of temporal variations in slope stability: the impact of hydrological processes. Geophysical research letters, 44: 4897-4 907.

Wunder S., 2005. Payment for environmental services: some nuts and bolts. Bogor: Cifor, (Occasional paper), 42, $24 \mathrm{p}$.

Zhang W., Ricketts T.H., Kremen C., Carney K., Swinton S.M., 2007. Ecosystem services and disservices to agriculture. Ecological economics, 64(2): 253-260. 



\section{$\mathrm{B}$ - Systèmes à base de cacaoyers}





\title{
Chapitre 5 \\ Les cacaoyères agroforestières au Centre et au Sud du Cameroun : diversité et dynamique
}

\author{
Michel I., Carrière S.-M., Manga Essouma F., Bihina M.A., \\ Blanchet A., Moisy C., Ngono F. et Levang P.
}

\begin{abstract}
Résumé. Produit d'une histoire ancienne, une agroforesterie cacaoyère multistrate et riche en biodiversité a été diffusée partout dans les régions du Centre et du Sud du Cameroun, de la périphérie des villes aux zones forestières les plus reculées. Créée il y a plus d'un siècle par les agriculteurs natifs de ces régions, cette agroforesterie a bénéficié, pendant longtemps, des appuis de l'État pour le développement de la cacaoculture. Depuis la fin des années 1980 et l'arrêt du soutien de l'État au secteur agricole, la production cacaoyère camerounaise a traversé une succession de crises et de booms impactant les profils et les stratégies des planteurs et, par conséquent, les structures et la composition en espèces d'arbres des cacaoyères. Une approche pluridisciplinaire, comparant quatre sites contrastés du point de vue biogéographique et socio-économique, nous a permis de déterminer et de caractériser les différents types de cacaoyères existant actuellement dans le Centre et le Sud du Cameroun. La diversité des plantations existe tant au niveau de la sous-région qu'au sein-même des différents sites d'étude. Elle est reliée aux histoires locales de plantation et reflète la diversité des planteurs. Selon les situations, les agroforêts cacaoyères multistrates semblables au modèle ancien se maintiennent, se transforment en partie ou continuent de s'étendre. Parallèlement, de nouvelles cacaoyères aux structures plus simples émergent et se développent, essentiellement portées par de nouveaux acteurs investissant dans la cacaoculture de rente.
\end{abstract}

\footnotetext{
Abstract. From urban margins to the most remote forest areas, multistrata and biodiversity-rich cocoa agroforestry systems emerged over time all over Centre and South Cameroon. For a long time, the development by local farmers of these original agroforestry systems benefitted from a strong support of the Cameroonian State. But the major economic crisis of the late 1980s and following adjustment plans put an end to the State's support to the agricultural sector. Then, cocoa growers of Centre and South Cameroon
} 
have followed different strategies and development paths, resulting in diversification of cocoa plantations in terms of structure and species composition of trees. This multidisciplinary study comparing four ecologically and socially contrasted sites identified and characterized the main different types of cocoa plantations existing in the Centre and the South Cameroon. A diversity of plantations was found both at the sub region level and within the different study sites, reflecting the diversity of types of farmers in relation to local planting histories. In some cases, multistrata cocoa agroforests have persisted as such or have been partially transformed, or even have expanded. In other cases, new simplified structures have appeared and diffused, generally promoted by new stakeholders investing non-agricultural capital in cocoa production.

\section{Introduction}

Au Cameroun, actuellement $5^{\mathrm{e}}$ pays producteur de cacao à l'échelle mondiale et $4^{\mathrm{e}}$ en Afrique (Icco, 2016), les régions Centre et Sud constituent le plus ancien bassin de production du pays. Ces régions partagent une histoire commune initiée à l'époque coloniale (1892) et relayée par une politique volontariste ${ }^{1}$ après l'Indépendance. Partout, des périphéries urbaines aux zones rurales forestières les plus reculées, s'est développée une cacaoculture agroforestière multistrate, portée par les familles natives de ces régions, aujourd'hui pluri-décennale voire centenaire (Santoir, 1992; Carrière, 2003; Jagoret et al., 2018). À la fin des années 1980, l'effondrement des prix des matières premières agricoles sur le marché international, dont le cacao, a provoqué une crise économique majeure au Cameroun, mettant fin au soutien de l'État au secteur agricole. Au début des années 2000, cette crise s'est interrompue avec la reprise à la hausse des cours mondiaux du cacao, entraînant une forte augmentation des surfaces cacaoyères entre 2003 et 2014 (Faostat, 2018). Selon les zones, cette succession d'événements a profondément modifié le secteur de la production cacaoyère. Les descendants des premiers planteurs se sont, pour la majorité, maintenus dans les zones de production initiales, reprenant les cacaoyères de leurs aïeux. D'autres planteurs se sont déplacés, allant des zones où la pression foncière était la plus forte, comme la Lékié, vers des zones reculées, forestières et moins densément peuplées, comme le Mbam et Kim, à la recherche de nouvelles terres. Sur ces sites d'accueil, ils se confrontent à de nouveaux contextes et à la présence de nouveaux acteurs désireux d'investir depuis peu dans la cacaoculture (Pedelahore, 2014). Ces éléments ont contribué à l'émergence d'une diversité de planteurs, aux stratégies variées. Faisant l'hypothèse que cette diversité de contextes et d'acteurs influe sur les systèmes techniques, et donc sur les types de plantations cacaoyères. Nous répondrons, dans ce chapitre, aux questions suivantes : les cacaoyères agroforestières multistrates sont-elles présentes partout? Sont-elles homogènes? Quelles sont les transformations à l'œuvre? Par quels types de planteurs sont-elles portées? Nous nous intéresserons également aux conduites techniques et aux performances de ces systèmes en termes de production de cacao.

1. Cette politique se traduisait par le soutien d'une recherche cacaoyère active et par la subvention des produits phytosanitaires, la fixation et le contrôle des prix du cacao payés aux producteurs, le soutien et le développement d'organismes d'appui technique et de vulgarisation allant jusqu'à la création en 1970 de la Sodecao (Société pour le développement du cacao) dotée de moyens importants. 


\section{\éthodologie et description des quatre sites d'étude}

Une approche pluridisciplinaire a été adoptée pour décrire les sites d'étude. Elle associe l'économie, l'ethnoécologie, l'écologie et l'agronomie. La démarche scientifique est basée sur une analyse comparative de situations contrastées, reflétant la diversité biogéographique et sociale rencontrée dans le Centre et le Sud du Cameroun. Elle a été mise en œuvre entre 2013 et 2015 sur un dispositif réunissant quatre sites (fig. 5.1).

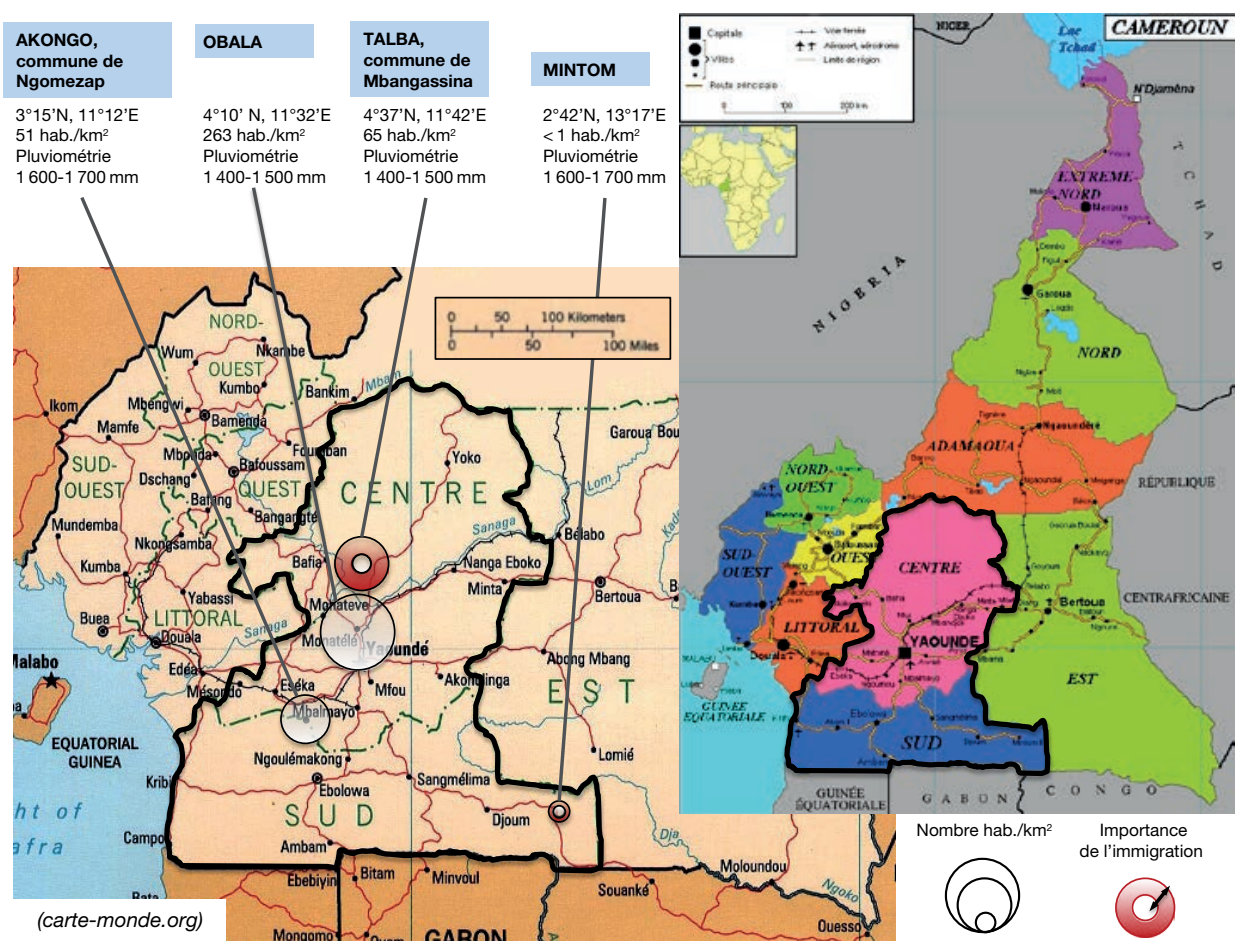

Figure 5.1. Localisation des quatre sites d'étude.

Source des données climatiques : Santoir et Bopda, 1995. Source des données démographiques : cvuc.com.

\section{Démographie et situtation géographique}

Tout en faisant varier l'importance des phénomènes migratoires, ce dispositif a été construit selon un gradient décroissant de densité démographique en s'éloignant de la capitale Yaoundé.

Le site d'Obala (département de la Lekié) présente la densité démographique la plus élevée, réunissant une population essentiellement Eton, composée des descendants des premiers planteurs de cacaoyers. Beaucoup parmi eux ont émigré dès le début des années 1970 vers d'autres zones à pression foncière plus faible.

Plus éloigné de Yaoundé, le site d'Akongo (département du Nyong et So'o) présente une densité démographique moyenne, sans phénomènes migratoires, réunissant une population d'origine essentiellement Éwondo. 
Le site de Talba (département du Mbam-et-Kim), portion forestière restée enclavée jusqu'à la construction d'un pont sur le fleuve Sanaga qui la borde (1979), fait partie des zones d'accueil de migrants originaires de la Lekié. Cette zone attire également de nouveaux acteurs d'origine urbaine. Elle réunit donc une population diversifiée autour d'un noyau d'origine, les Sanaga, aujourd'hui minoritaires et présente une densité démographique moyenne avec une population devenue à majorité Éton et Manguissa (Pedelahore, 2014).

Avec une très faible densité démographique, le site de Mintom (département du Dja-et-Lobo) est un front pionnier forestier récent et actif, en phase de désenclavement suite à la construction d'un axe goudronné; ce qui attire de nouveaux acteurs. La population majoritaire demeure celle d'origine - Fang et pygmées Baka rejointe à la fin des années 1980 par des familles Éton en provenance de la Lekié.

Les quatre sites se trouvent sur le plateau central camerounais, de topographie vallonnée, entre 500 et $850 \mathrm{~m}$ d'altitude. Les sols ferralitiques dominent, sous un climat subéquatorial de type guinéen à quatre saisons (Santoir et Bopba, 1995). Plus méridionaux, les sites d'Akongo et de Mintom sont dans le domaine de la forêt dense humide sempervirente guinéo-congolaise. Les sites d'Obala et de Talba, de pluviométrie annuelle plus faible, se trouvent dans le domaine de la forêt dense humide semi-caducifoliée.

\section{Échantillonnage des planteurs enquêtés et des cacaoyères}

Dans chaque site, des entretiens semi-directifs auprès de planteurs âgés et de chefs de village ont été menés afin d'identifier les dynamiques agricoles à l'œuvre, ainsi que la diversité des planteurs et des cacaoyères en présence. Un échantillon de planteurs a ensuite été construit et stratifié de manière à représenter leur diversité intrasite. À partir de 170 agriculteurs enquêtés (40 à 50 par site, pris dans différents villages et hameaux voisins), une typologie a été construite. Elle est basée sur les critères suivants : ancienneté familiale dans la cacaoculture (en nombre de générations); migration et ancienneté de cette migration (en nombre de générations); âge (héritage de cacaoyères); assise (ou superficie) cacaoyère in situ et statut foncier (superficie à dires d'acteurs des cacaoyères héritées, créées, confiées ou louées); main-d'œuvre principale mobilisée (familiale, salariée); activité principale cacaoyère ou autre (en termes de revenus à dires d'acteurs); trajectoire de vie géographique et professionnelle.

Soixante et onze cacaoyères choisies au sein de l'échantillon élargi de planteurs ont été inventoriées (20 à Obala, 20 à Mintom, 19 à Talba et 12 à Akongo), associant des cacaoyères relativement récentes (de 10 à 30 ans), anciennes ( $>30$ ans) et juvéniles ( $<10$ ans) dans le cas de nouvelles structures très spécifiques comme à Mintom.

\section{Indicateurs pour caractériser les cacaoyères}

Pour caractériser ces cacaoyères, nous avons utilisé des indicateurs classiques de structure (hauteur/individu, densité, surface terrière des arbres associés y compris les bananiers et les palmiers) et des indices de diversité (détermination des espèces 
associées et richesse spécifique ${ }^{2}$ ) (Sonwa et al., 2017). Les indicateurs concernant les arbres associés ont été mesurés dans une portion homogène de la plantation dans un carré de $50 \mathrm{~m} \times 50 \mathrm{~m}$, puis pour le peuplement cacaoyer dans un rectangle inclus dans le carré précédent de $20 \mathrm{~m} \times 50 \mathrm{~m}$. Seuls les arbres associés de diamètre $>5 \mathrm{~cm}$ ont été mesurés, ainsi que les cacaoyers de plus de trois ans. Les pratiques de gestion et de conduite des cacaoyères (fréquence annuelle des désherbages manuels et des traitements phytosanitaires, notamment fongicides ${ }^{3}$, date de création des cacaoyères, âge et variété des cacaoyers), ainsi que le rendement en cacao ont été renseignés à dires d'acteurs.

Trois strates ont été définies pour l'ensemble des cacaoyères du dispositif :

- la strate cacaoyère, de 1 à $8 \mathrm{~m}$ du sol, où se trouvent les peuplements cacaoyers, ainsi que quelques jeunes arbres fruitiers et forestiers (bananiers inclus);

- la strate intermédiaire, de 8 à $25 \mathrm{~m}$, où se retrouvent principalement les espèces fruitières épargnées ou plantées, ainsi que quelques jeunes arbres forestiers conservés (palmiers inclus);

- la strate émergente, de 25 à plus de $60 \mathrm{~m}$, où se retrouve l'essentiel des arbres forestiers et parfois des palmiers.

Pour comparer les caractéristiques des cacaoyères entre les sites, les variables mesurées ont été soumises à une analyse de variance (Anova) utilisant un modèle linéaire. En cas de différence significative mise en évidence par le test de Fisher (à p < 5\%), le test de TukeyHSD a été utilisé (logiciel R; version 3.5.0, 2018). Les compositions variétales des peuplements cacaoyers et les compositions spécifiques des peuplements associés sont données en pourcentage en fonction du nombre d'individus recensés sur l'ensemble des cacaoyères par site.

Par ailleurs, les cacaoyères sont classées selon la valeur de la surface terrière par hectare du peuplement associé, indicatrice de l'importance de ce peuplement dans les systèmes. Au Cameroun, pour des agroforêts cacaoyères complexes étudiés dans des zones similaires, différentes valeurs de surface terrière associée ont été relevées : entre 9 et $46 \mathrm{~m}^{2} / \mathrm{ha}$, données obtenues par Bisseleua et Vidal (2008); 17,6 m²/ ha, moyenne obtenue par Jagoret et al. (2017); 30,5 $\mathrm{m}^{2} /$ ha, moyenne indiquée par Sonwa et al. (2017).

Dans ce dispositif, quatre types de cacaoyères sont définies : «simple» pour une valeur de surface terrière associée inférieure à $8 \mathrm{~m}^{2} /$ ha; «moyenne» pour une valeur comprise entre 8 et $16 \mathrm{~m}^{2} /$ ha; «complexe» pour une valeur comprise entre 16 et $35 \mathrm{~m}^{2} / \mathrm{ha}$; «très complexe» pour une valeur supérieure à $35 \mathrm{~m}^{2} / \mathrm{ha}$.

\section{Mésultats}

Les cacaoyères complexes multistrates sont bien représentées dans notre dispositif. En totalité, nous avons recensé 135 espèces différentes d'arbres associés sur les quatre sites (85 espèces différentes à Mintom, 60 à Obala, 50 à Akongo et 45 à Talba) dont

2. Nombre d'espèces par unité de surface.

3. Un traitement fongicide correspond à un épandage à la dose recommandée par la Sodecao de 20 sachets dose de $50 \mathrm{~g} / \mathrm{ha}$, un sachet se diluant dans un pulvérisateur à dos de 15 litres. Sur l'année, la Sodecao recommande 10 traitements à réaliser pendant la phase de production de cabosses. 
$90 \%$ d'espèces forestières. En moyenne, les arbres associés sont présents à une densité de 112,7 individus/ha (tab. 5.1), avec une surface terrière de $21,5 \mathrm{~m}^{2} / \mathrm{ha}$. Si les arbres associés relevant de la strate cacaoyère représentent en nombre en moyenne 18,8\% de l'ensemble des individus associés $(37,4 \%$ pour ceux de la strate intermédiaire et $43,8 \%$ pour ceux de la strate émergente), leur contribution en surface terrière par hectare est faible, en moyenne 1,8\% de l'ensemble des individus associés (16,3\% pour ceux de la strate intermédiaire et $81,9 \%$ pour ceux de la strate émergente).

\section{Spécificités locales des cacaoyères}

\section{En nombre d'espèces et en surface terrière par hectare d'arbres associés}

Les cacaoyères de Mintom ont des peuplements associés significativement plus denses (individus/ha) et plus développés ( $\mathrm{m}^{2} / \mathrm{ha}$ ) que ceux des autres sites (tab. 5.1). Aussi, les arbres associés de la strate émergente contribuent-ils fortement à l'ensemble, avec une surface terrière et une densité significativement supérieure à très supérieure. Pour autant, les arbres associés relevant des strates cacaoyère et intermédiaire sont bien présents à Mintom, représentant des surfaces terrières par hectare significativement similaires à celles d'Akongo et de Talba (fig. 5.2). Les cacaoyères de Mintom sont de composition spécifique à la fois très riche et très forestière. Elles présentent le plus grand nombre d'espèces, dont 83,6\% sont forestières. Les deux espèces les plus représentées sont Terminalia superba (12,1\%) (espèce forestière) et Persea americana $(5,7 \%)$ (espèce fruitière).

En nombre d'espèces et en surface terrière par hectare d'arbres associés, les cacaoyères d'Obala présentent des valeurs plus faibles qu'à Mintom. Elles sont voisines de celles d'Akongo et de Talba (tab. 5.1). En revanche, les cacaoyères d'Obala se distinguent par l'importance de leurs strates cacaoyères et intermédiaires (fig. 5.2), représentant des surfaces terrières par hectare significativement supérieures à celles des autres sites. Ces valeurs sont liées à des densités élevées des arbres associés dans ces deux strates, notamment dans la strate cacaoyère avec une composante fruitière importante. Seulement 33,8\% des arbres associés recensés à Obala sont des espèces forestières; ce qui se traduit par la surface terrière moyenne par arbre associé la plus faible. Les deux espèces les plus représentées sont fuitières : Persea americana $(17,5 \%)$ et Dacryodes edulis (12,7\%).

Les cacaoyères de Talba se distinguent par une densité d'arbres associés significativement inférieure à celle des autres sites (tab. 5.1). Il s'agit majoritairement d'espèces forestières (81,7\% des individus recensés). Les arbres associés sont particulièrement peu présents dans la strate intermédiaire, avec une densité significativement inférieure aux autres sites. La strate émergente présente une faible densité, mais une surface terrière élevée associée aux espèces les plus représentées sur le site, toutes deux forestières : Terminalia superba $(20,5 \%)$ et Ceiba pentadra $(5,1 \%)$.

Les cacaoyères d'Akongo sont à la fois forestières et fruitières, avec toutefois une densité d'arbres associés dans la strate émergente élevée. Près de $74 \%$ des arbres associés recensés sont des espèces forestières. Les deux espèces les plus représentées sont Terminalia superba (13,5\%) et Dacryodes edulis (10,9\%). 


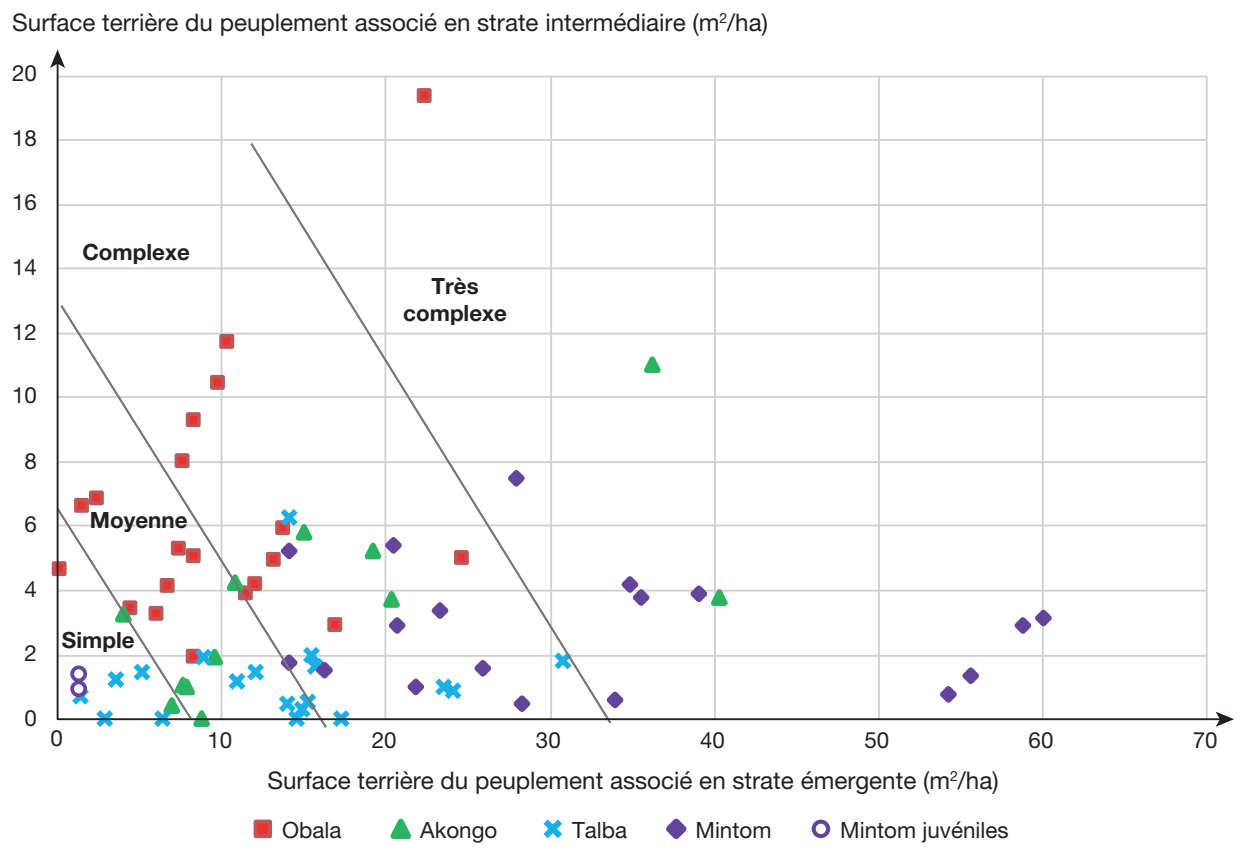

Figure 5.2. Répartition des cacaoyères en fonction de la surface terrière du peuplement arboré associé selon les strates et en distinguant les sites (données issues de nos enquêtes menées de 2013 à 2015).

Structure simple, surface terrière toutes strates confondues $<8 \mathrm{~m}^{2} /$ ha; structure moyenne, surface terrière entre 8 et $16 \mathrm{~m}^{2} / \mathrm{ha}$; structure complexe, surface terrière entre 16 et $35 \mathrm{~m}^{2} / \mathrm{ha}$; structure très complexe, surface terrière $>35 \mathrm{~m}^{2} / \mathrm{ha}$. ( $\mathrm{n}=71$, dispositif d'enquêtes Centre et Sud du Cameroun, 2013-2015).

\section{Ancienneté des cacaoyères}

Les cacaoyères les plus anciennes se trouvent sur les sites historiques sans immigration : à Obala, âgées en moyenne de 61 ans (tab. 5.1), la plus âgée ayant 113 ans; à Akongo, âgées de 55 ans en moyenne, la plus âgée ayant 78 ans. À Mintom, la moyenne d'âge des cacaoyères est de 34 ans, la plus âgée ayant 48 ans. Les cacaoyères de Talba sont les plus jeunes (28 ans en moyenne), la plus âgée ayant 56 ans.

Dans les cacaoyères anciennes d'Obala, le peuplement cacaoyer fait l'objet d'un fort renouvellement comme le montre l'âge moyen des cacaoyers, similaire à celui des cacaoyères de Talba et de Mintom, tout en étant significativement inférieur à celui des cacaoyères d'Akongo. Ce renouvellement se retrouve dans la composition variétale de ces peuplements : la variété ancienne 'Amelonado' (ou 'Cacao allemand') domine dans les cacaoyères d'Akongo où elle représente $98,4 \%$ des cacaoyers recensés. Elle est moins représentée à Mintom (79,3\%), à Obala $(65,3 \%)$ et encore moins à Talba $(26,1 \%)$.

\section{Rendement en cacao et conduite technique}

Si les cacaoyères des quatre sites ont des densités cacaoyères similaires avec une moyenne de 1399 individus/ha, elles ont des rendements en cacao significativement 


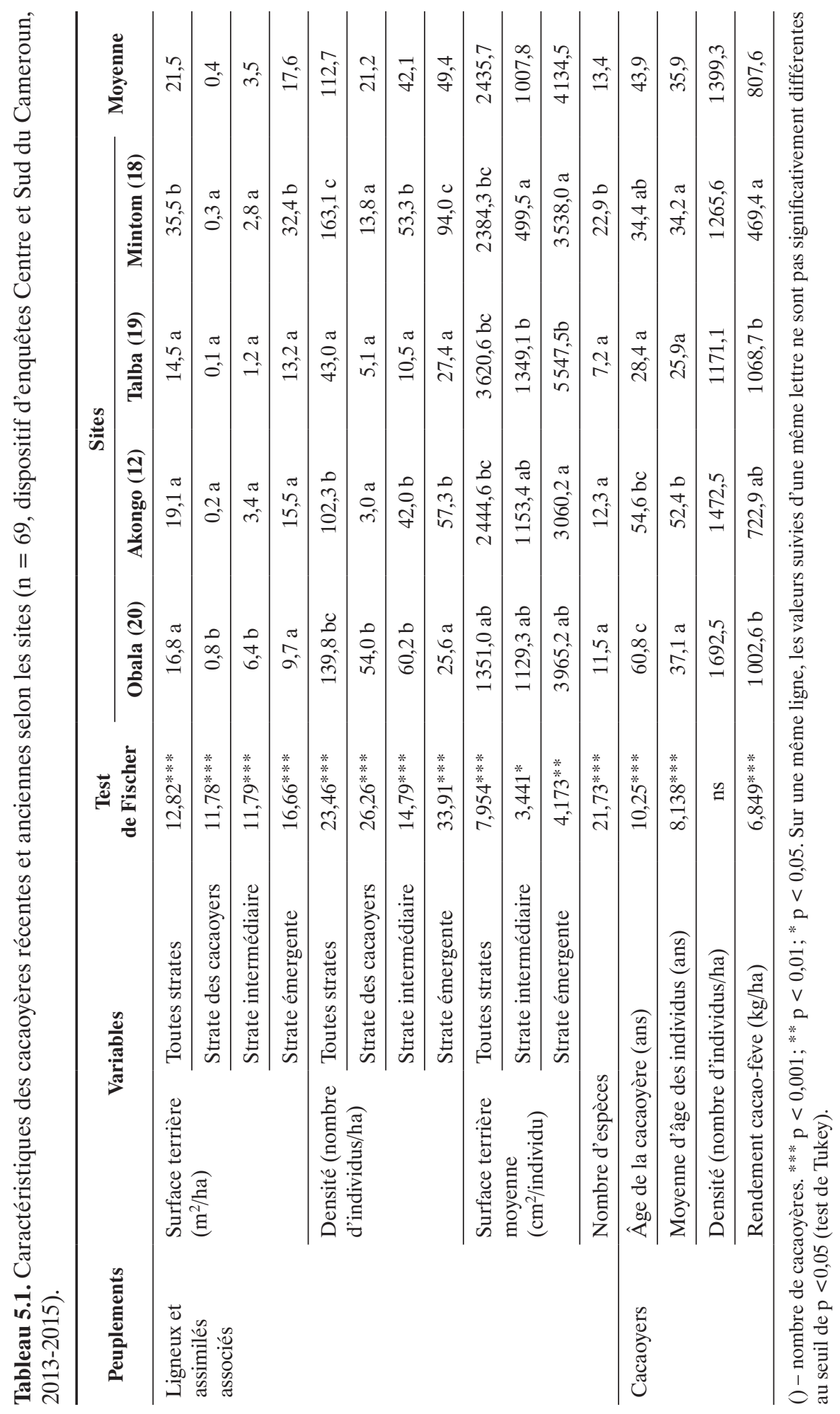


différents. Les plus faibles rendements sont obtenus à Mintom, suivis de près par les rendements obtenus à Akongo. Les plus élevés sont obtenus à Obala et Talba (tab. 5.1).

En termes de conduite technique, les cacaoyères d'Obala et de Talba sont désherbées deux à trois fois et traitées cinq à six fois par an. À Akongo, les cacaoyères sont désherbées deux fois et traitées quatre fois par an. À Mintom, certaines cacaoyères sont désherbées une à deux fois et traitées cinq fois par an, d'autres ne sont désherbées qu'une fois par an et non traitées.

\section{La diversité intrasite : diversité des planteurs, des cacaoyères et dynamiques à l'œuvre}

En plus des différences significatives observées entre les sites, les cacaoyères présentent aussi une diversité de structures au sein-même des sites, comme le montre la figure 5.2. Le tableau 5.2 affiche la répartition des types de cacaoyères au sein d'un site, en distinguant les cacaoyères récentes des anciennes; ce qui permet de mettre en exergue les dynamiques. Par ailleurs, ce tableau croise les types de cacaoyères avec les types de planteurs rencontrés dans chacun des sites.

Tableau 5.2. Croisement des types de cacaoyères $(n=71)$ et des types de planteurs (dispositif d'enquêtes Centre et Sud du Cameroun, 2013-2015)

\begin{tabular}{|c|c|c|c|c|c|c|c|c|c|}
\hline \multirow[b]{2}{*}{ Site } & \multirow[b]{2}{*}{ Type de planteurs } & \multicolumn{4}{|c|}{$\begin{array}{c}\text { Type de cacaoyères }<30 \text { ans } \\
\text { (nombre) }\end{array}$} & \multicolumn{4}{|c|}{$\begin{array}{c}\text { Type de cacaoyères }>\mathbf{3 0} \text { ans } \\
\text { (nombre) }\end{array}$} \\
\hline & & 产 & 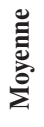 & $\frac{\mathscr{z}}{\tilde{e}}$ & 承 & 冚 & 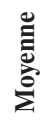 & 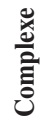 & 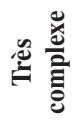 \\
\hline \multirow[t]{3}{*}{ Obala } & CacaoHéritActif & 1 & 1 & 1 & & & 3 & 3 & 1 \\
\hline & CacaoSecond & & & & & & 2 & 3 & \\
\hline & CacaoRetraite & & & & & & 2 & 3 & \\
\hline Akongo & CacaoHéritActif & 1 & 1 & & & 1 & 4 & 3 & 2 \\
\hline \multirow[t]{5}{*}{ Talba } & CacaoHéritActif & & 1 & & & 1 & 2 & 5 & \\
\hline & Jeune avant héritage & & 2 & 1 & & & & & \\
\hline & CacaoMigrant & & 2 & 1 & & & & & \\
\hline & Locataire & 2 & & & & & & & \\
\hline & Petit investisseur & 2 & & & & & & & \\
\hline \multirow[t]{4}{*}{ Mintom } & CacaoSecond & & & & & & & 4 & 2 \\
\hline & CacaoMigrant & & & 3 & 3 & & & & \\
\hline & Locataire & & & & & & & & 4 \\
\hline & Petit investisseur & 2 juv. & & & & & & & \\
\hline
\end{tabular}




\section{Dans les sites historiques sans immigration (Obala et Akongo)}

Le site d'Akongo présente une grande homogénéité de planteurs de cacaoyers correspondant, selon les cas, à la deuxième ou troisième génération de planteurs. Ces planteurs sont classés dans la catégorie des héritiers actifs mobilisant essentiellement de la main-d'œuvre familiale (Cacao Hérit Actif). Les 40 planteurs de notre échantillon élargi ont en moyenne 52 ans et exploitent 3,7 ha de cacaoyères en moyenne chacun en plus des cultures vivrières, avec un maximum de surface de 9 ha. La plupart de ces héritiers ont une trajectoire de vie qui les a conduits temporairement hors du village pour étudier ou exercer des activités professionnelles. Avant leur retour et la prise en main des cacaoyères héritées, celles-ci étaient souvent confiées et exploitées en métayage; ce mode de faire-valoir n'a pas modifié leur structure et se prolonge en période de prix bas du cacao.

La catégorie de planteurs héritiers se retrouve à Obala. Mais la densité démographique plus élevée et la proximité de la capitale Yaoundé font émerger deux autres types de planteurs :

- ceux qui, bien qu'ayant pris en main les cacaoyères héritées, ont conservé une activité prépondérante au cacao — fonctionnaires de l'administration, salariés d'une activité urbaine ou commerçants - (Cacao Second);

- ceux qui ont repris tardivement les cacaoyères héritées, au moment de la retraite d'une autre activité en ville (Cacao Retraite).

Comme à Akongo, les jeunes non héritiers se mettent au service de leurs aînés temporairement absents ou trop âgés, via un métayage ou plus rarement une location de cacaoyères à l'année. Les 50 planteurs de notre échantillon élargi à Obala ont en moyenne 60 ans et exploitent 2 ha en moyenne de cacaoyères chacun, avec un maximum de 3,5 ha.

Les 27 cacaoyères anciennes situées à Akongo et à Obala ont connu des périodes de transition de durée variable avec métayage. Elles étaient toutes à l'origine structurées de manière «complexe» ou «très complexe». Reprises en main par les héritiers, elles ont été maintenues ainsi (15) ou simplifiées jusqu'à devenir «moyennement» complexes (11) voire «simple»(1). Les cacaoyères ainsi simplifiées sont toutes associées à des cacaoyères maintenues complexes chez les mêmes agriculteurs. Les cinq cacaoyères récentes de notre dispositif ont été mises en place par des héritiers actifs dans le but d'agrandir leurs superficies. Elles sont de structure «simple» (2) et «moyenne» (2), associées à des cacaoyères anciennes maintenues complexes. Une seule est de structure «complexe».

La plupart des agriculteurs héritiers enquêtés à Akongo ont la possibilité d'étendre encore la superficie de leurs cacaoyères sur des réserves foncières. Celles-ci sont cependant insuffisantes pour permettre l'installation de cacaoyères pour des jeunes n'ayant pas encore hérité. À Obala, seulement $10 \%$ des planteurs enquêtés déclarent encore disposer de réserves foncières, mais celles-ci sont faibles (en moyenne 2 ha). D'importants phénomènes d'émigration sont à l'œuvre au départ d'Obala : soit les héritiers se déplacent en confiant leurs cacaoyères aux bons soins d'une première épouse épaulée par des métayers, soit ils favorisent l'installation de leurs fils sur des fronts pionniers cacaoyers en zone forestière à Talba ou au sud du pays. 


\section{Dans les sites d'accueil de migrants}

\section{À Talba}

On retrouve les migrants de la Lekié à Talba, où ils ont créé et créent encore de nouvelles cacaoyères. Du fait de l'ancienneté de cette migration toujours active, une première génération de planteurs migrants coexiste avec une deuxième qui a déjà hérité de cacaoyères. Les premiers sont regroupés dans le type des migrants issus d'autres zones cacaoyères (Cacao Migrant); les seconds viennent grossir le type des héritiers de Talba, composé également des premières familles de planteurs (Cacao Hérit Actif). Du fait de la disponibilité des terres, les jeunes n'ayant pas encore hérité peuvent commencer leurs propres plantations in situ, formant un nouveau type (Jeune avt héritage). Il existe un type spécifique de grands investisseurs (Gd invest.) à Talba, apparu à la fin des années 1980, développant des surfaces cacaoyères importantes avec des moyens ad hoc reposant sur l'emploi de main-d'ouvre salariée. Le type de petits investisseurs (Petit Invest.) regroupe des urbains souvent originaires de Yaoundé, arrivés dans les années 1990, fuyant la crise économique qui touchait alors tous les secteurs de l'économie camerounaise, venant investir dans le cacao des revenus modestes, faisant appel également à de la main-d'œuvre salariée. Ces grands et petits investisseurs dynamisent le marché foncier à Talba, ainsi que le salariat agricole qui attire des migrants issus d'autres régions du Cameroun (principalement du Nord-Ouest) et, dans une moindre mesure, des pays frontaliers (Tchad, Nigeria, etc.). Ces migrants se spécialisent dans les travaux de la cacaoculture.

Les 40 planteurs de notre échantillon élargi de Talba, toutes catégories confondues, ont en moyenne 50 ans et exploitent chacun en moyenne 11 ha de cacaoyères, le maximum étant 40 ha. Les héritiers associent des cacaoyères anciennes héritées de structures «complexes» (5) à des cacaoyères anciennes de structures simplifiées «moyennes» (2) et «simples » (1), ainsi qu'à une cacaoyère récente «moyenne » (1). Les jeunes avant héritage et les migrants mettent en place des cacaoyères «simplifiées moyennes» (4) et «complexes» (2). Les cacaoyères de structure «simple» non associées à d'autres sont récentes (4) et aux mains des grands et petits investisseurs urbains.

\section{À Mintom}

À Mintom, les terres peuvent encore s'acquérir auprès des populations en échange d'une somme symbolique ou de cadeaux. Arrivés plus récemment qu'à Talba, les migrants de la Lekié sont classés dans le type des migrants issus d'autres zones cacaoyères (Cacao Migrant). Quant aux premières familles de planteurs de cacaoyers de Mintom, elles forment un type spécifique aux zones forestières éloignées (Cacao Second). Ces acteurs étaient initialement des chasseurs-cueilleurs cultivant un peu de vivrier, devenus planteurs de cacaoyers lors des campagnes de plantation initiées par l'État au début des années 1960, puis poursuivies par la Sodecao. Suite à la mise en veille de cette société en 1990, ils ont quasiment stoppé l'entretien de leurs plantations, ne réalisant que quelques interventions manuelles. Cependant, ils ont conservé des activités de chasse importantes, prépondérantes à l'entretien des cacaoyères. Par ailleurs, le désenclavement récent de la zone a généré l'essor de nouvelles activités (commerces, administrations locales, orfèvrerie...), 
attirant de nouveaux acteurs originaires d'autres régions du Cameroun. Certains choisissent d'investir dans une petite cacaoculture de type patronal employant de la main-d'œuvre constituée de pygmées Baka sédentarisés (Petit Invest). D’autres migrants originaires du Nord-Ouest (communément appelés «Bamenda») commencent à affluer à Mintom de façon saisonnière puis de manière permanente. Ils sont attirés par les cacaoyères peu entretenues des premiers planteurs de Mintom qu'ils prennent en location pour une à plusieurs saisons, formant un nouveau type (locataire). Ils investissent en travail et en intrants sans être autorisés à modifier la structure des plantations louées.

Toutes catégories confondues, les planteurs de notre échantillon élargi à Mintom ont en moyenne 45 ans et exploitent chacun en moyenne 7,5 ha de cacaoyères, 26 ha au plus. Toutes les cacaoyères des premiers planteurs de Mintom sont anciennes et de types «complexe» à «très complexe», qu'elles soient aux mains de locataires Bamenda ou non. Les plantations récentes sont le fruit des familles migrantes de la Lekié, elles sont également de type «complexe» à «très complexe». Les cacaoyères de type «simple», présentes uniquement au stade juvénile, sont spécifiques des petits investisseurs qui émergent à Mintom, ne développant que ce type de cacaoyère.

\section{"Discussion}

Dans les sites de cacaoculture historique d'Obala et d'Akongo, les types de planteurs sont peu nombreux. Ils contribuent à la diversification des structures des cacaoyères héritées, complexes au départ, en les simplifiant en partie. Les cacaoyères les plus anciennes sont présentes à Obala, là où la densité démographique est aussi la plus forte. Elles sont riches en arbres fruitiers, avec un peuplement cacaoyer renouvelé et jeune, et sont conduites de façon relativement intensive. Lobservation de ces cacaoyères anciennes à Obala montre qu'il existe une marge d'évolution dans la conduite des cacaoyères à Akongo, à commencer par le renouvellement du peuplement cacaoyer.

Sur les sites de plantation plus récents de Mintom et de Talba, les types de planteurs sont plus variés. Les arbres associés au cacaoyer sont surtout forestiers, avec plusieurs types de structures selon les situations et les acteurs. À Talba où la densité démographique augmente, la tendance est à la simplification des structures des nouvelles cacaoyères (moins de strates et moins d'arbres par strate); ce qui va de pair avec l'utilisation de nouvelles variétés de cacaoyers conduites de façon relativement intensive. À Mintom où la pression démographique reste faible, les cacaoyères demeurent complexes, qu'elles soient entretenues par les migrants de la Lekié ou des héritiers in situ. Dans les sites de Talba et Mintom, il existe des cacaoyères de structure simple, récentes ou juvéniles, portées par des acteurs investissant du capital d'origine non agricole. Leur objectif est essentiellement le gain financier à grande échelle et, si possible, à court terme. Ces cacaoyères sont implantées avec de nouvelles variétés hybrides qui demandent plus de lumière. Les arbres associés sont quand même présents dans ces nouvelles cacaoyères (fig. 5.2).

À Mintom, nous observons un réservoir d'anciennes cacaoyères complexes avec une forte proportion d'arbres forestiers, peu, voire pas, entretenues. Les pratiques et les rendements en cacao observés dans les cacaoyères louées et donc reprises en 
main par les Bamenda prouvent qu'elles peuvent être réactivées en une seule saison. Dans notre échantillon, les six cacaoyères récoltées et entretenues par les premiers planteurs, désherbées une fois par an et non traitées, ont des rendements moyens de $300 \mathrm{~kg} / \mathrm{ha}$ de cacao marchand. Les six cacaoyères louées par les Bamenda, désherbées une à deux fois et traitées cinq fois par an, ont des rendements moyens de $556 \mathrm{~kg} / \mathrm{ha}$; ce qui est très proche en termes de pratiques et de rendement (moyenne de $558 \mathrm{~kg} / \mathrm{ha}$ ) des six cacaoyères récentes des migrants issus de la Lekié.

\section{Conclusion}

Les cacaoyères agroforestières observées présentent une grande diversité de structures et de compositions. Dans le site le plus ancien (Obala), ces cacaoyères agroforestières ont une forte composante fruitière. Si elles se simplifient en partie, il n'en est pas moins vrai qu'elles se maintiennent parce qu'elles sont entretenues et conduites de façon relativement intensive. Parallèlement, les mêmes familles qui participent à leur maintien in situ alimentent des courants migratoires et sont à l'origine de nouvelles implantations cacaoyères ailleurs dans le pays, exportant ainsi leurs systèmes et leurs savoir-faire ou le transformant selon les contextes. Sur le front pionnier de Mintom, les planteurs reproduisent et étendent les agroforêts multistrates conduits de façon peu intensive. À Talba, où l'espace d'accueil est plus ancien et où le brassage de populations est important, les planteurs créent de nouvelles cacaoyères aux structures plus simples et de composition très forestière. Ils mobilisent davantage les nouvelles variétés de cacaoyer conduites de façon relativement intensive. À Akongo, autre site historique où les familles poursuivent le développement de leurs activités cacaoyères dans un espace qui devient limité, sans perspectives d'émigration pour le moment. Les cacaoyères multistrates se maintiennent ou se simplifient en partie, et elles sont conduites de façon peu intensive. Quant aux cacaoyères présentant des structures simples dès l'origine, récentes ou juvéniles, elles se développent essentiellement sur les fronts pionniers (Talba et Mintom) où elles sont portées par de nouveaux acteurs ayant des capacités d'investissement. Dans le site forestier enclavé (Mintom), il existe encore un nombre important de cacaoyères agroforestières anciennes et inactives dont l'entretien pourrait être repris.

\section{" Bibliographie}

Bisseleua D.H., Vidal B.S., 2008. Plant biodiversity and vegetation structure in traditional cocoa forest gardens in Southern Cameroon under different management. Biodiversity conservation, 17 : 1821-1 835. http://dx.doi.org/10.1007/s10531-007-9276-1.

Carrière S., 2003. Les orphelins de la forêt. Pratiques paysannes et écologie forestière (Ntumu, SudCameroun). Montpellier, France : IRD Éditions.

Jagoret P., Michel I., Todem Ngnogue H., Lachenaud P., Snoeck D., Malézieux E., 2017. Structural characteristics determine productivity in complex cocoa agroforestry systems. Agronomy for sustainable development, 37-60. https://doi.org/10.1007/s13593-017-0468-0.

Jagoret P., Todem Ngnogue H., Malézieux E., Michel I., 2018. Trajectories of cocoa agroforests and their drivers over time: lessons from the Cameroonian experience. European journal of agronomy, 101: 183-192. https://doi.org/10.1016/j.eja.2018.09.007. 
Pedelahore P., 2014. Systèmes agroforestiers à cacaoyers et transition capitaliste : l'exemple du Centre-Cameroun. Bois et forêts des tropiques, (321) : 55-66.

Santoir C., 1992. Sous l'empire du cacao : étude diachronique de deux terroirs camerounais. Paris : Orstom.

Santoir C., Bopda A., 1995. Atlas régional Sud-Cameroun. Paris : Orstom.

Sonwa D.J., Weise S.F., Nkongmeneck B.A., Tchatat M., Janssens M.J.J., 2017. Structure and composition of cocoa agroforests in the humid forest zone of Southern Cameroon. Agroforestry systems, 91(3): 451-470. http://dx.doi.org/10.1007/s10457-016-9942-y. 


\title{
Chapitre 6 \\ Compromis entre séquestration de carbone, conservation et productivité dans les systèmes agroforestiers à base de cacaoyers au Centre-Cameroun
}

\author{
Saj S., Jagoret P., Mvondo Sakouma K., Essobo J.-D., \\ Bouambi E. et Todem-Ngogue $\mathrm{H}$.
}

\begin{abstract}
Résumé. Les systèmes agroforestiers à base de cacaoyers du Centre-Cameroun présentent des niveaux de stockage de carbone (C), de conservation d'espèces d'arbres et de production de cacao intéressants. Les services de conservation et de stockage de carbone peuvent s'y révéler synergiques, mais ils semblent tous les deux antagonistes des niveaux de production supérieurs à une tonne/ha de fèves de cacao marchand. Le stockage de carbone peut néanmoins être découplé de la conservation car dans ces systèmes, il est principalement réalisé par de très grands arbres. Ainsi, un nombre réduit d'individus peut suffire à atteindre des stocks de $100 \mathrm{t} \mathrm{C/ha.} \mathrm{La} \mathrm{conservation} \mathrm{des} \mathrm{espèces} \mathrm{ligneuses} \mathrm{apparait,}$ quant à elle, dépendante du niveau de multifonctionnalité que les agriculteurs attendent de leurs systèmes agroforestiers à base de cacaoyers. Par ailleurs, les systèmes ayant des niveaux de stockage de carbone élevés ou moyens semblent être en mesure de soutenir une production cacaoyère intéressante au-delà de 40 ans $(0,75$ à $1 \mathrm{t} / \mathrm{ha}$ cacao marchand). Les travaux présentés permettent de distinguer trois groupes de systèmes agroforestiers à base de cacaoyers : simples, peu durables et peu multifonctionnels, mais avec de bonnes capacités de production de cacao; complexes, plus durables avec des niveaux de stockage de carbone et de production de cacao intéressants, mais peu de capacité de conservation; très complexes avec de bons niveaux de stockage de $\mathrm{C}$ et de conservation, mais limités en production de cacao. Ces trois groupes, correspondant à des situations réelles, ils permettent de mieux cibler les intervalles dans lesquels des compromis entre services écosystémiques sont acceptables.
\end{abstract}

Abstract. Cocoa-based agroforestry systems (cAFS) from Central-Cameroon showed interesting levels of carbon storage (C), tree species conservation and cocoa production. 
Carbon storage and species conservation services may prove to be synergistic but both were found antagonistic to cocoa production levels above $1 \mathrm{t} / \mathrm{ha}$. Carbon storage could also be decoupled from conservation because in these systems the former was mainly carried out by very large trees. Hence, a small number of individuals could support stocks of $100 \mathrm{t} / \mathrm{ha}$. Species conservation appeared to be dependent on the level of multifunctionality that farmers expect from their cAFS. In addition, cAFS with high or average carbon storage levels seemed able to sustain an interesting cocoa production ( 0.75 to $1 \mathrm{t} / \mathrm{ha}$ ) beyond 40 years. The herein highlighted studies allow to distinguish 3 groups of cAFS: (i) simple, poorly sustainable, poorly multifunctional, yet with good cocoa production capacity; (ii) complex, more sustainable with interesting levels of carbon storage and cocoa production but little conservation capacity; (iii) very complex with good levels of storage and conservation abilities but limited in cocoa production. These three groups, corresponding to real situations, make it possible to better target the intervals in which it is possible to obtain acceptable trade-offs between ecosystem services.

\section{" Contexte et enjeux autour des systèmes agroforestiers à base de cacaoyers}

La production mondiale de cacao est aujourd'hui de l'ordre de 4 à 4,5 millions de tonnes par an (Icco, 2016). Elle est en grande partie (environ 80\%) le fruit du travail de petits agriculteurs dont les vergers excèdent rarement les cinq-six hectares (Jagoret, 2011). Cependant, la cacaoculture est ou a été une cause majeure de déforestation dans certaines régions du monde, notamment en Afrique (Cleaver, 1992). En effet, les conditions économiques d'exploitation et les modes de gestion des cacaoyères mises en place par exemple en Côte d'Ivoire ou au Ghana - les deux premiers pays producteurs mondiaux — ont incité, de façon cyclique, beaucoup d'agriculteurs à migrer vers des fronts pionniers. Ces migrants ont ainsi profité d'une «rente forestière » pour la création à moindre coût de nouvelles cacaoyères, leur évitant ainsi le surcoût du renouvellement des anciens vergers en perte de rendement (Ruf, 1995).

Les systèmes agroforestiers à base de cacaoyers, qui mêlent cacaoyers et plusieurs autres espèces pérennes ayant des fonctions et usages divers, sont souvent qualifiés de «complexes» (Clough et al., 2009; Somarriba et al., 2013). Cette complexité semble limiter la déforestation et l'érosion de la biodiversité qui sont associées à la cacaoculture, tout en maintenant une production durable de cacao.

Dans la région Centre du Cameroun, qui produit du cacao depuis plus d'une centaine d'années, des systèmes agroforestiers à base de cacaoyers en forêt sont installés après abattis-brulis partiel du couvert forestier. Lors du défrichement, quelques arbres forestiers sont conservés (fruitiers indigènes, espèces médicinales et espèces ligneuses) pour assurer un ombrage aux jeunes cacaoyers et pour leur valeur économique. Lassociation de cultures annuelles (Zea mais, Arachis hypogaea, Xanthosoma sagittifolium, etc.) et de cultures pluriannuelles (Manihot esculenta et Musa paradisiaca) est également pratiquée sur quelques cycles. Dans le même temps, les agriculteurs introduisent plusieurs espèces fruitières dans le système de culture (Elaeis guineensis, Citrus sinensis, Dacryodes edulis, Cola acuminata, etc.) Ces espèces croissent en association avec les cacaoyers et les espèces forestières conser- 
vées (Jagoret et al. 2009). Ces systèmes sont exploités avec très peu d'intrants par rapport au modèle conventionnel recommandé (Jagoret et al., 2011). Leur durabilité semble basée sur le maintien d'un équilibre stratégique entre les peuplements cacaoyers et les communautés d'arbres associés. Cependant, le potentiel productif de ces systèmes est peu étudié et mal évalué aujourd'hui. Par ailleurs, le CentreCameroun abrite encore des espaces forestiers protégés, proposés à la protection ou non protégés (Agter, 2012) pouvant servir de références essentielles en particulier à l'évaluation du niveau des services de stockage de carbone (C) et de conservation de la biodiversité qu'offrent les systèmes agroforestiers à base de cacaoyers. Ces informations seront alors très utiles pour la prise de décision concernant les politiques à mener dans les zones de production cacaoyère.

Dans un premier temps, ce chapitre résume les principaux résultats du projet Safse (Compromis entre production et autres services écosytémiques dans les systèmes agroforestiers, Cirad/IRD) sur les systèmes agroforestiers à base de cacaoyers du Centre-Cameroun pour «trois services» : conservation d'espèces ligneuses, stockage de carbone $(\mathrm{C})$ et production de cacao. Dans un deuxième temps, il traite des compromis et des synergies entre ces services. Afin de travailler sur des gradients d'association intéressants, trois zones ont été particulièrement étudiées afin d'obtenir l'échantillonnage le plus large possible en termes d'âges des cacaoyères et de niveaux d'ombrage pour des conditions pédoclimatiques relativement proches (fig. 6.1; Jagoret, 2011; Pédelahore, 2012).
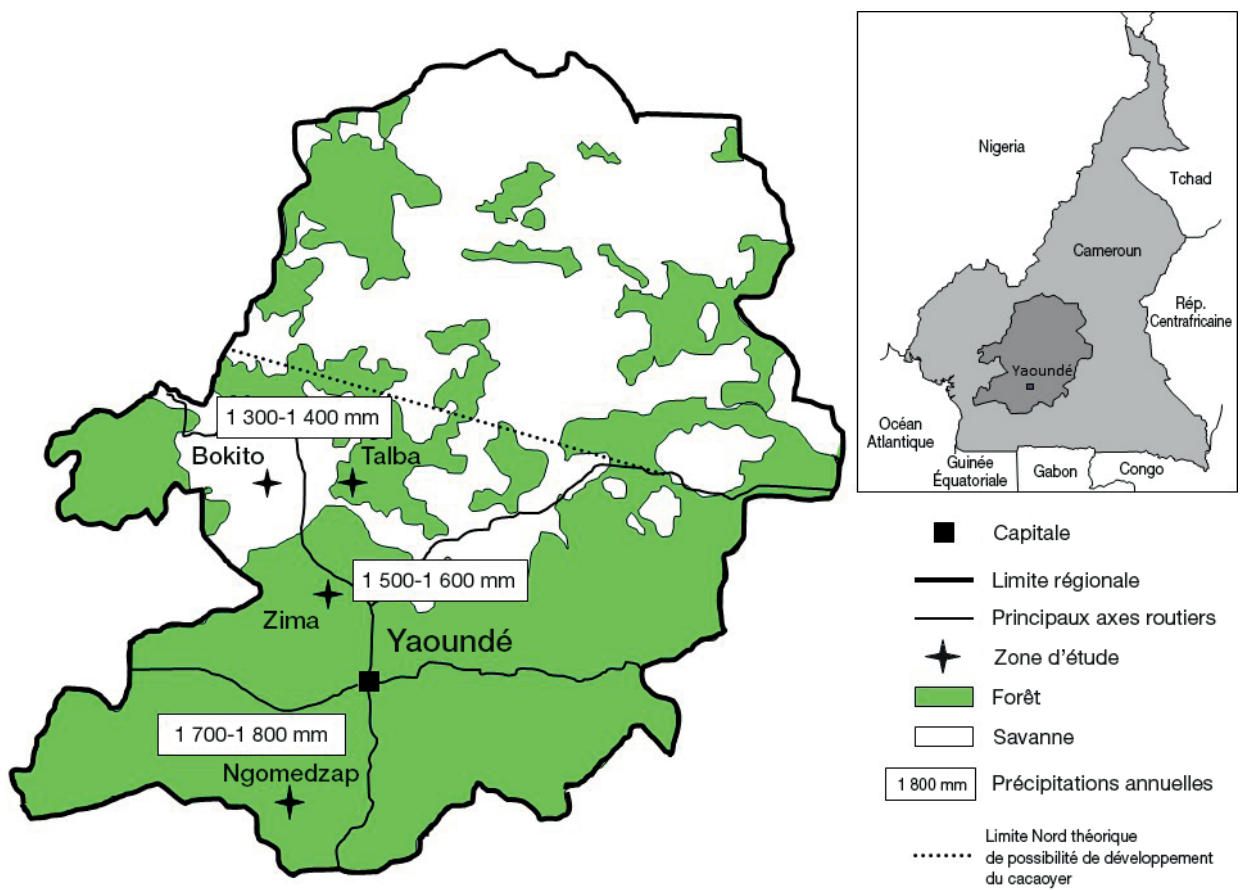

Figure 6.1. Carte de la région Centre du Cameroun et localisation des trois zones d'échantillonnage (Bokito et Talba, Zima, Ngomedzap) des systèmes agroforestiers à base de cacaoyers. 


\section{" Les services écosystémiques étudiés au Centre-Cameroun}

\section{Le stockage de carbone}

Le projet Safse s'est particulièrement intéressé au stockage de carbone (C) dans la biomasse ligneuse des systèmes agroforestiers à base de cacaoyers du Centre-Cameroun. Dans les résultats présentés ici, le stock de carbone des sols et des végétaux non ligneux n'est pas pris en compte. La méthode de calcul du stockage de carbone dans la biomasse arborée de ces systèmes a reposé sur des inventaires d'arbres et de leurs dimensions (hauteur, diamètre du tronc à $130 \mathrm{~cm}$ de hauteur), ainsi que de l'application de relations allométriques (Saj et al., 2013).

Lors de la mise en place de la cacaoyère en forêt, un déstockage important de carbone intervient. Puis, tout au long de la croissance des cacaoyers et des arbres associés, le stockage de carbone augmente jusqu'à atteindre parfois un niveau proche de celui d'un système forestier (fig. 6.2; Saj et al., 2017a). Il s'avère cependant que ce stockage est principalement le fait des grands arbres ayant un diamètre supérieur à $30 \mathrm{~cm}$ qui sont favorisés tout au long de la vie de la cacaoyère. Le stockage de carbone dans la biomasse des peuplements cacaoyers reste marginal, il est comparable au stockage des arbres associés de petite taille (au diamètre $<30 \mathrm{~cm}$ ). La dynamique de (re)stockage devient positive environ 20 ans après la mise en place des systèmes agroforestiers à base de cacaoyers, puis elle stagne après 60 ans.

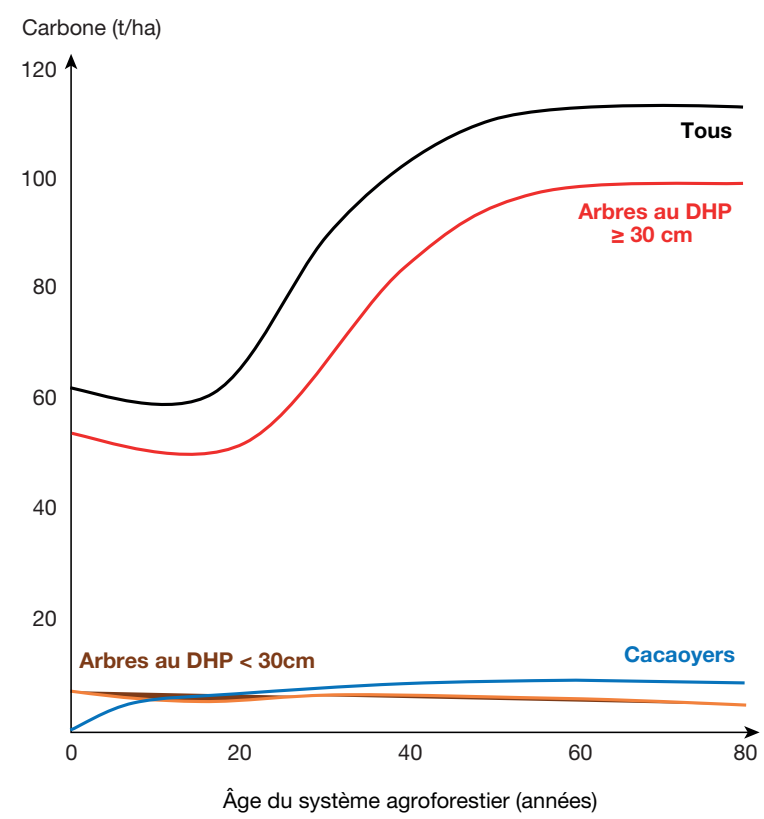

Figure 6.2. Dynamique de stockage de carbone dans la biomasse ligneuse de systèmes agroforestiers à base de cacaoyers au Centre-Cameroun.

Moyennes obtenues à partir d'un échantillonnage de 144 parcelles (Saj et al., 2017a). DHP : diamètre à hauteur de poitrine. 
La pérennisation sur le long terme du stockage de carbone dans les systèmes agroforestiers à base de cacaoyers dépend donc du devenir des grands arbres associés aux cacaoyers. Dans la stratégie de mise en place puis d'entretien de ces systèmes, ces grands arbres occupent plusieurs fonctions pour les agriculteurs. Ils permettent un ombrage plus léger et plus homogène de la cacaoyère comparé à celui des arbres de taille plus modeste. Certains de ces arbres possèdent également des caractéristiques régulièrement considérées comme positives par les planteurs pour la production cacaoyère : amélioration de la fertilité du sol ou mitigation de la ressource en eau (Ceiba pentandra, Ficus mucoso) (Jagoret et al., 2014). Enfin, ces grands arbres présentent souvent une qualité de bois qui les rendent intéressants à la vente ou à l'utilisation en tant que bois d'œuvre (Entandrophragma cylindricum, Terminalia superba) (Saj et al., 2017a). Même pour les systèmes les plus simples — densités inférieures ou proches de 30 arbres associés/ha - , ces grands arbres sont toujours présents. A contrario, la densité des arbres de moindre diamètre (inférieur à $30 \mathrm{~cm}$ ) a tendance à diminuer avec l'âge de la cacaoyère. Ce sont ces artbres qui sont davantage éliminés lors de la simplification des parcelles agroforestières (Saj et al., 2017a).

En conclusion, la pérennisation des niveaux élevés de stockage de carbone rencontrés dans les systèmes agroforestiers à base de cacaoyers du Centre-Cameroun dépend, bien qu'indirectement, de la pérennisation et de la gestion à long terme des cacaoyers en leur sein. La substitution de la composante cacaoyère par une autre culture, à l'image de ce qui est observé en Côte d'Ivoire avec l'hévéa par exemple (Ruf, 1995), représente actuellement le plus grand risque de perte de carbone dans ces systèmes.

\section{La conservation des essences forestières}

Dans le cadre du projet Safse, l'étude des systèmes agroforestiers à base de cacaoyers du Centre-Cameroun s'est focalisée sur les capacités de conservation de leur communauté ligneuse. Une des spécificités de ces systèmes du Centre-Cameroun réside dans le fait qu'ils sont composés d'un grand nombre d'espèces associées aux cacaoyers. Ces espèces sont représentées par des nombres d'individus assez réduits, surtout lorsqu'il s'agit d'essences forestières (Jagoret, 2011; Saj et al. 2017a). Notre analyse a porté sur 11,4 ha de systèmes agroforestiers à base de cacaoyers et 1,4 ha de témoins forestiers (forêts secondaires sans cacaoyers). Plus de 3000 arbres associés aux cacaoyers ont été comptabilisés dans 147 cacaoyères agroforestières, pour un nombre total de 192 espèces. Cette richesse spécifique est cependant à pondérer en fonction du degré de complexité des systèmes observés rencontrés et des zones étudiées (Bokito, Ngomedzap et Talba). En moyenne, les systèmes agroforestiers à base de cacaoyers comportent 10 à 30 essences associées/ha pour des densités variant de 30 à plus de 200 arbres/ha. Néanmoins, ces chiffres sont inférieurs aux estimations réalisées pour les systèmes forestiers de cette région : plus de 135 espèces inventoriées en forêt pour une surface échantillonnée d'environ dix fois moindre. Mais il est tout de même intéressant de noter que la proportion d'espèces ayant un enjeu de conservation (selon le statut de la liste rouge de l'IUCN) est de l'ordre de $20 \%$, à la fois dans les forêts et dans toutes les classes d'âge des systèmes agroforestiers à base de cacaoyers (Saj et al., 2017a). 
Il apparaît donc que les systèmes agroforestiers à base de cacaoyers ne sont pas en mesure de pérenniser le milieu forestier. Cependant, comparés à d'autres systèmes de culture, ils représentent un des systèmes agricoles les plus divers qui soient. En outre, ils présentent l'avantage de permettre la conservation de nombreuses espèces rencontrées dans les zones forestières du Centre-Cameroun. Par conséquent, ces systèmes possèdent des caractéristiques utiles à des actions conservationnistes et peuvent constituer des zones tampons efficaces. En effet, ils permettent la préservation tout au long de leur vie de nombreux arbres appartenant à des espèces confrontées à des enjeux de conservation. Il a d'ailleurs été récemment émis l'hypothèse que pour certaines de ces espèces, le potentiel de conservation dans les systèmes agroforestiers à base de cacaoyers pourrait se révéler plus intéressant que dans les forêts exploitées (Abada Mbolo et al., 2016). Enfin, ces systèmes comprennent également d'autres espèces dont les enjeux de conservation ne sont pas encore déterminés.

Le potentiel écologique des systèmes agroforestiers à base de cacaoyers du CentreCameroun dépend néanmoins de la densité des arbres associés aux cacaoyers. Ainsi, contrairement au stockage de carbone, la simplification de ces systèmes conduirait à une diminution drastique de ce potentiel. Dans les parcelles agroforestières échantillonnées, lorsque le nombre d'individus associés passe de plus de 200 à moins de 50 individus/ha (différence entre les systèmes agroforestiers complexes et simples), le nombre d'espèces associées a diminué de plus de $70 \%$ et les indices de diversité alpha ${ }^{1}$ d'environ $50 \%$ (Saj et al., 2017a). Aussi, le potentiel de conservation des systèmes agroforestiers à base de cacaoyers dépend de leur nature multifonctionnelle, les arbres qui y sont conservés possédant chacun un ou plusieurs usages (Jagoret et al., 2014). Si l'usage des arbres associés est uniquement orienté vers l'ombrage et le maintien de la fertilité du sol pour la cacaoculture, comme dans le cas d'une faible densité d'arbres associés ( $<50$ individus/ha), les espèces à usages locaux et traditionnels sont amenées à disparaître, par exemple les espèces médicinales Drypetes staudtii et Massularia acuminata. Or, la plupart de ces espèces présentent un enjeu de conservation reconnu ou indéterminé à ce jour.

Ainsi, la pérennisation des capacités de conservation en essences forestières des systèmes agroforestiers à base de cacaoyers au Centre-Cameroun dépend de la stabilité sur le long terme des modes d'exploitation extensifs et multifonctionnels qui y sont pratiqués (Jagoret et al., 2018).

\section{La production de cacao des systèmes agroforestiers}

Lestimation de la production de cacao des systèmes agroforestiers à base de cacaoyers du Centre-Cameroun a consisté à compter 4 à 5 fois/an les nouvelles cabosses de taille supérieure à $10 \mathrm{~cm}$ de longueur (Saj et al., 2017b). Les données de production obtenues correspondent à des rendements que l'on peut qualifier d' «accessibles », c'est-à-dire le rendement maximum que l'on pourrait obtenir si toutes les cabosses dépassant $10 \mathrm{~cm}$ de longueur atteignaient la maturité et restaient indemnes d'accidents et de pression parasitaire jusqu'à la récolte. Ce rendement accessible donne

1. La diversité $\alpha$ est une mesure du nombre d'espèces présentes dans un habitat uniforme de taille fixe à un temps donné. 
donc une idée du potentiel productif in itinere de ces systèmes en permettant de s'abstraire des effets de certaines pratiques des agriculteurs (désherbage et application de pesticides et de fongicides notamment). En effet, ces pratiques peuvent influencer le rendement final en cacao (Saj et al., 2017b). En revanche, le rendement accessible intègre les effets des modalités de mise en place et de gestion des systèmes agroforestiers à base de cacaoyers que les agriculteurs raisonnent sur le moyen et long terme (Jagoret et al., 2018).

La production sous couvert forestier est dépendante du degré de compétition, d'une part, entre arbres associés et cacaoyers et, d'autre part, entre les cacaoyers eux-mêmes lorsque la densité de plantation est trop importante. Nous avons tenté d'évaluer l'effet de la compétition entre le peuplement cacaoyer et les arbres associés sur le rendement en utilisant la notion de surface terrière ${ }^{2}$ (Saj et al., 2017b). Cette approche a permis d'illustrer pour la première fois les rendements en fonction d'un indicateur d'interactions interspécifiques : le rapport de la surface terrière des cacaoyers/la surface terrière totale de la communauté (STC/STT).

Nos résultats montrent une relation significativement positive entre le STC/STT et les rendements accessibles en cacao $\left(\mathrm{R}^{2}=0,45 ; \mathrm{p}=0,02\right)$. De manière générale, il peut être considéré qu'effectivement, la présence d'arbres associés concurrence les cacaoyers. Ces résultats montrent également que la présence d'autres arbres n'explique pas l'ensemble des variations. Ainsi, les rendements dépendent également de l'âge de la parcelle cacaoyère (Saj et al., 2017b). Enfin, les niveaux de rendement accessible obtenus tendent à montrer qu'une meilleure gestion au cours du cycle - par exemple en termes de stratégies de protection ou de prophylaxie sanitaire pourrait grandement améliorer les rendements réels obtenus ou déclarés, qui se situent régulièrement entre 250 et $750 \mathrm{~kg} /$ ha de cacao marchand (Jagoret et al., 2011). Cette amélioration de la gestion pourrait se révéler bien plus payante et durable qu'une diminution de la densité des arbres associés dans les systèmes agroforestiers à base de cacaoyers. Par ailleurs, il est remarquable que, malgré un effort d'échantillonnage important, nous n'ayons pas recensé des systèmes agroforestiers à base de cacaoyers âgés de plus 60 ans dont la surface terrière relative des cacaoyers était supérieure à $50 \%$ (Saj et al., 2017b). Même s'il reste à valider, ce résultat suggère une limite à ne pas dépasser en termes de diminution du nombre d'arbres associés pour une production sur le long terme et à bas niveau d'intrants.

\section{" Les interactions entre services}

\section{Interactions entre conservation des essences forestières et stockage de carbone}

Le stockage de carbone $(\mathrm{C})$ et la conservation des essences forestières sont deux services écosystémiques plus ou moins directement liés au nombre d'arbres associés aux cacaoyers dans les systèmes agroforestiers à base de cacaoyers. Il n'est donc pas

2. Surface terrière : surface de la section d'un arbre à hauteur de poitrine $(130 \mathrm{~cm})$. Ici, on a sommé les surfaces terrières du peuplement cacaoyer d'une parcelle échantillonnée. La surface terrière totale de la parcelle correspond à la somme de l'ensemble des arbres constituant la parcelle échantillonnée. 
surprenant d'observer une relation positive entre ces deux services et, par conséquent, des évolutions de type synergétique entre stockage de carbone et nombre d'espèces associées $\left(\mathrm{R}^{2}=0,36\right.$ et $\mathrm{R}^{2}=0,635$ en fonction de l'indice de richesse spécifique $^{3}$ ou de la diversité choisie). Cependant, cette relation n'est pas aussi simple qu'il y paraît. Comme elle dépend fortement de la nature et de la taille des individus associés aux cacaoyers, tous les systèmes agroforestiers à base de cacaoyers étudiés ne rentrent pas dans ce schéma. Par rapport à la moyenne observée, certains intègrent des communautés d'espèces associées constituées de peu d'individus, mais de grande taille; d'autres possèdent de nombreux individus, mais à la taille modeste. Ces cas démontrent que la synergie n'est pas forcément systématique et que certains choix dans la conduite technique des systèmes agroforestiers à base de cacaoyers dissociant les deux services pourraient être effectués (Saj et Jagoret, 2017).

\section{Interactions entre rendement en cacao et stockage de carbone}

Les rendements accessibles en cacao des systèmes agroforestiers à base de cacaoyers sont négativement corrélés au stockage de carbone. Cette relation non linéaire présente une variabilité importante qui est fonction du niveau de stockage de carbone (fig. 6.3a). En effet, il apparaît d'une part que moins le stockage de carbone est important, plus la variabilité du rendement est importante. D'autre part, des rendements inférieurs à $1 \mathrm{t} / \mathrm{ha}$ de cacao marchand peuvent être obtenus assez indépendamment du niveau de stockage de carbone. Ainsi, l'obtention d'un rendement élevé ( $>1,5 \mathrm{t} / \mathrm{ha}$ ) n'est possible que pour un niveau de stockage en carbone inférieur à 100-150 t C/ha. À contrario, un niveau de stockage supérieur à $150 \mathrm{t} \mathrm{C} /$ ha ne semble pas être compatible avec un rendement accessible supérieur à $750 \mathrm{~kg} /$ ha de cacao marchand. En d'autres termes, un rendement supérieur à $1,5 \mathrm{t} /$ ha de cacao marchand n'est possible que lorsque la compétition avec les arbres se situe en deçà d'un certain seuil. Par ailleurs, il semble que dans les conditions actuelles de gestion, la durée maximum d'exploitation de ces systèmes plus simples soit d'environ 40 ans (Saj et al., 2017b).
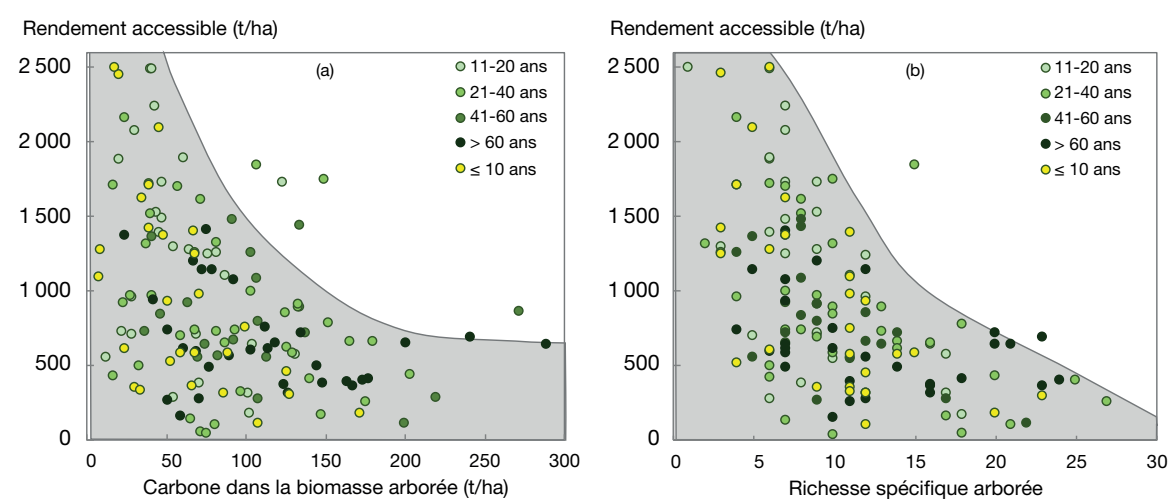

Figure 6.3. Évolution du rendement en cacao accessible en fonction (a) du carbone (C) de la biomasse arborée associée; (b) de la richesse spécifique de la communauté arborée associée. Le fond gris représente l'espace dans lequel (a) $96 \%$ ou (b) $98 \%$ des systèmes agroforestiers à base de cacaoyers étudiés se situent.

3. Richesse spécifique : nombre d'espèces par unité de surface. 
La majorité des systèmes agroforestiers à base de cacaoyers échantillonnés sont dans une situation intermédiaire à celles décrites plus haut. Il apparaît donc que des marges de manœuvre importantes existent et pourraient être mobilisées dans l'objectif de mieux concilier rendement et stockage de carbone malgré une relation globalement négative.

\section{Interactions entre rendement en cacao et conservation des essences forestières}

En ce qui concerne les capacités de conservation des espèces forestières au sein des systèmes agroforestiers à base de cacaoyers, étudiées ici à partir de la richesse spécifique de la communauté associée, il y a également une relation négative avec le rendement accessible en cacao. De même que pour le stockage de carbone, il existe une très grande variabilité des niveaux de richesse spécifique pour un rendement donné. Des rendements supérieurs à $1 \mathrm{t} / \mathrm{ha}$ de cacao marchand ne semblent pas pouvoir être obtenus pour un nombre d'espèces associées supérieur à 10. Par contre, il semble possible d'atteindre des rendements de l'ordre de $750 \mathrm{~kg} / \mathrm{ha}$ de cacao marchand pour des richesses spécifiques allant jusqu'à 20-23 espèces associées. Dès lors, une réflexion équivalente à celle effectuée sur le stockage de carbone peut être engagée pour une meilleure conciliation entre richesse spécifique et rendement.

Cependant, on doit prendre en compte la notion d'usage des espèces associées aux cacaoyers. En effet, comme démontré par Jagoret et al. (2014), le choix des essences associées est effectué par les agriculteurs en fonction des usages et des bénéfices qu'ils souhaitent en tirer. Les systèmes agroforestiers à base de cacaoyers ayant maintenu les usages les plus traditionnels sont les systèmes les plus riches et les plus divers, mais leurs rendements en cacao sont les moins élevés. Au contraire, les systèmes montrant des usages des essences associées moins divers présentent des niveaux de rendement plus élevés (Saj et al., 2017b). Enfin, comparativement au stockage de carbone, les marges de manœuvre apparaissent faibles pour des niveaux de richesses spécifiques élevés (fig. 6.3b). Ces niveaux sont liés à des densités d'arbres associés élevés généralement supérieures à 100 individus/ha, et donc à une compétition pour la ressource plus importante avec le peuplement cacaoyer.

\section{Interactions entre rendement en cacao, stockage de C et conservation des essences forestières}

Lanalyse des relations entre ces trois variables montre que le stockage de carbone et la conservation des espèces forestières semblent assez synergiques, alors que ces deux variables sont deux antagonistes au rendement accessible. La figure 6.4 présente ces relations en fonction des niveaux de rendements accessibles observés.

Lobtention de rendements supérieurs à $1,5 \mathrm{t} / \mathrm{ha}$ de cacao marchand n'est peu ou pas compatible avec des niveaux de stockage supérieurs à $100 \mathrm{t} C /$ ha et une richesse spécifique supérieure à 10 espèces. Par ailleurs, ces rendements semblent difficilement atteignables au-delà de 40 ans de cacaoculture. Cela est vraisemblablement dû à la diminution des capacités productives des cacaoyers, voire de l'augmentation de la pression sanitaire dans le temps comme c'est le cas des mirides (Babin, 2009; Jagoret et al., 2011). 


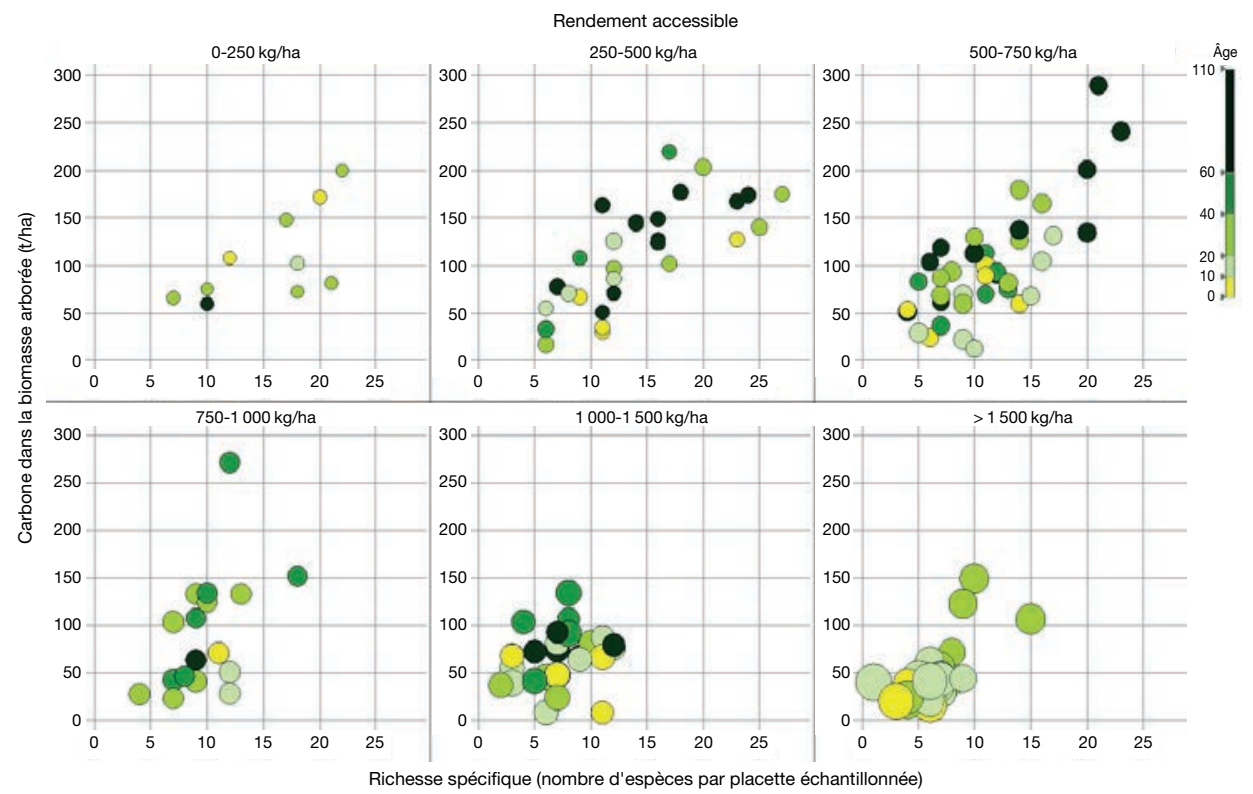

Figure 6.4. Relations entre la richesse spécifique (abscisse) et le carbone dans la biomasse arborée (ordonnée) selon le rendement accessible (différents graphes et taille des points), et l'âge des systèmes agroforestiers à base de cacaoyers (gradient de couleur).

\section{Le groupe 1 : rendements accessibles supérieurs à $1 \mathrm{t} /$ ha de cacao marchand}

Les niveaux moyens de stockage de carbone et de richesse spécifique sont comparables pour des rendements compris entre $1 \mathrm{t} /$ ha et $1,5 \mathrm{t} / \mathrm{ha}$ ce cacao marchand et semblent pouvoir être maintenus au-delà de 60 ans d'exploitation dans certains cas. Ces systèmes constituent le groupe 1 pour lequel des rendements accessibles seraient supérieurs à $1 \mathrm{t} / \mathrm{ha}$ de cacao marchand. Les objectifs seraient au minimum pour le stockage de carbone $80 \mathrm{~kg} \mathrm{C} / \mathrm{ha}$ et pour la conservation des espèces 10 espèces/ ha sur le long-terme. Ce groupe 1 peut constituer un référentiel technique pour des systèmes agroforestiers à base de cacaoyers que les agriculteurs souhaiteraient simples. Leurs arbres associés n'auraient que peu d'utilité autre que celle de l'ombrage et du maintien de la fertilité du sol.

\section{Le groupe 2 : rendements entre $750 \mathrm{~kg} / \mathrm{ha}$ et $1000 \mathrm{~kg} / \mathrm{ha}$ de cacao marchand}

Pour les parcelles dont le niveau de rendement en cacao est situé entre $750 \mathrm{~kg} / \mathrm{ha}$ et $1000 \mathrm{~kg} / \mathrm{ha}$, les niveaux de stockage de carbone paraissent plus importants que ceux du groupe 1. Mais ils ne sont pas clairement associés à des niveaux de richesse spécifique supérieurs. Ainsi, ces systèmes plus «stockeurs» que «conservateurs» pourraient former un second groupe (groupe 2). Dans ce groupe 2, la communauté associée comporte plus d'individus que dans le groupe 1. La compétition interspécifique y est plus importante pour les cacaoyers. Cette plus grande densité ne 
semble pas être associée à plus d'usages que dans le groupe 1 et peut s'expliquer par une gestion différente de la croissance des cacaoyers, de la fertilité des sols et de la pression parasitaire. Ce groupe 2 peut constituer un référentiel technique pour des systèmes agroforestiers à base de cacaoyers que les agriculteurs souhaitent toujours orientés vers une bonne production cacaoyère, mais dont les moyens sont plus limités que ceux du groupe 1. Les arbres associés n'auraient pas d'autre utilité que celle de l'ombrage et du maintien de la fertilité du sol. Les niveaux de stockage de carbone sur le long terme pouvant être visés par ce groupe 2 seraient compris entre 100 et $150 \mathrm{t} \mathrm{C} / \mathrm{h}$ a pour un niveau minimum 10 espèces/ha.

\section{Le groupe 3 : rendements accessibles inférieurs à $750 \mathrm{~kg} / \mathrm{ha}$ de cacao marchand}

Pour les rendements accessibles inférieurs à $750 \mathrm{~kg} / \mathrm{ha}$, il apparaît que la variation du niveau de stockage de carbone s'accompagne d'une variation de la richesse spécifique. La répartition entre classes d'âges de la cacaoyère semble être assez similaire entre les niveaux de rendement 250-500 et 500-750 kg/ha de cacao marchand. Ainsi, ce groupe 3 des systèmes agroforestiers, à base de cacaoyers à faible rendement, comporte aussi des systèmes moins performants que ceux des groupes 1 et 2 pour les trois variables étudiées. Cette diversité de situations est le reflet de l'hétérogénéité de structure et de gestion de la communauté des espèces d'arbres associées. Par ailleurs, on remarque qu'au sein de ce groupe, de nombreuses cacaoyères présentent des niveaux de stockage de carbone et de richesse spécifique équivalents à ceux des groupes 1 et 2, associés à des niveaux de rendements accessibles relativement faibles. Ce constat questionne la gestion de ces systèmes et souligne un besoin d'accompagnement des agriculteurs pour améliorer ces niveaux.

\section{W Conclusion}

Les résultats présentés dans ce chapitre démontrent la complexité des liens entre production, conservation et stockage de carbone dans les systèmes agroforestiers à base de cacaoyers du Centre-Cameroun. Ils soulignent une claire synergie entre les deux services de régulation et l'antagonisme de ces derniers avec le rendement accessible en cacao. Lanalyse des compromis entre ces trois services a permis de dégager trois groupes de systèmes agroforestiers à base de cacaoyers dans l'échantillonnage effectué :

- le premier groupe est composé de systèmes plutôt simples avec des rendements supérieurs à $1 \mathrm{t} /$ ha de cacao marchand. Il permettrait d'obtenir à moyen terme des niveaux de stockage de carbone et de richesse spécifique faibles à moyens pouvant atteindre respectivement 80-100 t C/ha et 10 espèces ha;

- le deuxième groupe est constitué de systèmes dont la densité de la communauté associée, un peu plus importante, entraîne une compétition avec le peuplement cacaoyer accrue et n'autorise plus que des rendements compris entre 750 et $1000 \mathrm{~kg} /$ ha. Ces systèmes, à la richesse spécifique équivalente à ceux du groupe 1 , semblent pouvoir atteindre des niveaux de stockage de carbone plus élevés à long terme situés au-delà des 100 t C/ha, voire 150 t C/ha; 
- le troisième groupe rassemble des systèmes plus hétérogènes. Ces systèmes semblent les plus prometteurs concernant les services de régulation étudiés en montrant des potentialités de rendements plus faibles compris entre 500 et $750 \mathrm{~kg} / \mathrm{ha}$ de cacao marchand. Les parcelles sont composées de plus de 15 espèces différentes et stockent plus de $150 \mathrm{t} \mathrm{C/ha}$. Ce groupe comporte également des systèmes dont tous les niveaux de services sont faibles, démontrant la nécessité d'une amélioration de leur gestion.

Dans certains pays, l'incompatibilité présumée entre les objectifs agronomiques, économiques et écologiques des systèmes agroforestiers favorise ou a favorisé l'émergence de mécanismes de paiement pour services environnementaux. Leur objectif est de soutenir, au moins en partie, des systèmes moins ou peu intensifs en se concentrant sur la rétribution d'externalités positives jusqu'alors ignorées (Rapidel et al., 2011). Ces mécanismes semblent intéressants du fait des niveaux de services relevés pour les systèmes agroforestiers à base de cacaoyers du CentreCameroun. Cependant, avant une quelconque mise en place de paiements pour services environnementaux, il sera nécessaire d'étudier avec une méthodologie adaptée les compromis entre les services que l'on souhaite rétribuer afin de déterminer les niveaux souhaitables et atteignables (Andreotti et al., 2018; Rapidel et al., 2015). Il sera également nécessaire de travailler à la forme des paiements pour services environnementaux la plus acceptable socialement et économiquement par les populations concernées.

\section{" Bibliographie}

Abada Mbolo M.M., Zekeng J.C., Mala W.A., Fobane J.L., Djomo Chimi C., Ngavounsia T., Nyako C.M., Menyene L.F.E., Tamanjong Y.V., 2016. The role of cocoa agroforestry systems in conserving forest tree diversity in the Central region of Cameroon. Agroforestry systems, 90: 577-590.

Andreotti F., Mao Z., Jagoret P., Speelman E.N., Gary C., Saj S., 2018. Exploring management strategies to enhance the provision of ecosystem services in complex smallholder agroforestry systems. Ecological indicators, 94 : 257-265.

Agter, 2012. Carte du domaine forestier du Cameroun, Août 2012, d'après le travail de cartographie du World resources institute. www.agter.org/bdf/fr/corpus_chemin/fiche-chemin-124.html (consulté le 09/03/2017).

Babin R., 2009. Contribution à l'amélioration de la lutte contre le miride du cacaoyer Sahlbergella singularis Hagl. (Hemiptera : Miridae). Influence des facteurs agroécologiques sur la dynamique des populations du ravageur. Thèse de doctorat, Université Montpellier III-Paul Valéry, France, $202 \mathrm{p}$.

Cleaver K., 1992. Deforestation in the western and central African rainforest: the agricultural and demographic causes, and some solutions. In: Cleaver K., Munasinghe M., Dyson M., Egli N., Penker A., Wencelius F. (eds). Conservation of west and central African rainforests. Washington: World bank, IUCN, 65-78.

Clough Y., Faust H., Tschnarke T., 2009. Cacao boom and bust: sustainability of agroforests and opportunities for biodiversity conservation. Conservation letters, 2(5): 197-205.

Icco, 2016. Icco annual report 2013/2014.

Jagoret P., Todem Ngnogue H., Bouambi E., Battini J.L., Nyassé S., 2009. Diversification des exploitations agricoles à base de cacaoyer au Centre Cameroun : mythe ou réalité ? Biotechnologie, agronomie, société et environnement, 13(2) : 271-280. 
Jagoret P., 2011. Analyse et évaluation de systèmes agroforestiers complexes sur le long terme : application aux systèmes de culture à base de cacaoyer au Centre-Cameroun. Thèse de doctorat. Montpellier SupAgro, France. 288 p.

Jagoret P., Kwesseu J., Messie C., Michel-Dounias I., Malézieux E., 2014. Farmers' assessment of the use value of agrobiodiversity in complex cocoa agroforestry systems in central-Cameroon. Agroforestry systems, 88: 983-1000.

Jagoret P., Michel-Dounias I., Malézieux E., 2011. Long-term dynamics of cocoa agroforests: a case study in central Cameroon. Agroforestry systems, 81: 267-278.

Jagoret P., Snoeck D., Bouambi E., Todem Ngnogue H., Nyassé S., Saj S., 2018. Key management practices keeping cacao agroforestry systems effective on the very long-term: evidences brought by long-lived plantations of Central-Cameroon. Agroforestry systems, 92: 1185-1199.

Pédelahore P., 2012. Stratégies d'accumulation des exploitants agricoles : l'exemple des cacaoculteurs du Centre Cameroun de 1910 à 2010. Université Toulouse II Le Mirail, Toulouse, p. 443.

Rapidel B., Le Coq J.F., DeClerck F.A.J., Beer J., 2011. Measurement and payment for ecosystem services from agriculture and agroforestry: new insights from the neotropics. In: Rapidel B., Le Coq J.F., DeClerck F.A.J., Beer J., eds. Ecosystem services from agriculture and agroforestry: measurement and payment. Londres, Royaume-Uni: Earthscan, p. 377-396.

Rapidel B., Ripoche A., Allinne C., Metay A., Deheuvels O., Lamanda N., Blazy J.M., ValdesGomez H., Gary C., 2015. Analysis of ecosystem services trade-offs to design agroecosystems with perennial crops. Agronomy for sustainable development, 35: 1373-1390.

Ruf F., 1995. Booms et crises du cacao. Les vertiges de l'or brun. Paris: Karthala, 459 p.

Saj S., Jagoret P., 2017. Traditional cacao agroforestry in Central-Africa can provide both respectable yields and levels of ecosystem services. In: Icco (ed.) International symposium on cocoa research (ISCR). Lima: Icco.

Saj S., Durot C., Mvondo-Sakouma K., Tayo Gamo K., Avana-Tientcheu M.L., 2017a. Contribution of companion trees to long-term tree conservation, carbon storage and agroforest sustainability: a functional analysis of the diversity in cacao plantations of Central Cameroon. International journal of agricultural sustainability, 15(3): 282-302.

Saj S., Jagoret P., Etoa L.E., Fonkeng E.E., Tarla J., Essobo J.D., Mvondo-Sakouma K., 2017 b. Lessons learned from long term analysis of cocoa yield and stand structure in agroforestry systems of Central-Cameroon. Agricultural systems, 156: 95-104.

Saj S., Jagoret P., Todem Ngogue H., 2013. Carbon storage and density dynamics of associated trees in three contrasting Theobroma cacao agroforests of Central-Cameroon. Agroforestry systems, 87: 1309-1320.

Somarriba E., Lachenaud P., 2013. Successional cocoa agroforests of the Amazon-Orinoco-Guiana shield. Forests, trees and livelihoods, 22(1): 51-59. 



\title{
Chapitre 7 \\ Structures spatiales et régulation des bioagresseurs des agroforêts à cacaoyers au Cameroun et au Costa Rica
}

\author{
NGo BIENG M.A., Gidoin C. ET TeN Hoopen G.M.
}

Résumé. Dans ce travail, nous montrons que la structure spatiale des individus (arbres et cacaoyers) au sein d'agroforêts à cacaoyers influence l'intensité d'attaque de bioagresseurs des cacaoyers. À l'échelle de la parcelle, des organisations spatiales régulières ou aléatoires d'arbres forestiers réduisent les intensités d'attaques de la moniliose au Costa Rica et des mirides au Cameroun. À l'échelle de l'individu, le nombre de voisins cacaoyers (à $3,7 \mathrm{~m}$ ) et fruitiers (à 4,3 m) influence négativement l'intensité d'attaque de la moniliose au Costa Rica. Nos résultats révèlent l'importance de la structure spatiale dans la description des agroforêts tropicaux et la compréhension des mécanismes influençant la régulation agroécologique des bioagresseurs au sein de ces systèmes complexes. La gestion de la structure spatiale des individus dans les systèmes agroforestiers pourrait ainsi constituer un levier intéressant pour le contrôle agroécologique des bioagresseurs.

\begin{abstract}
Here we show that spatial tree structure within cacao agroforests influences pest and disease attack intensity of cacao trees. At the plot scale, regular or random spatial organizations of forest trees reduced pest and disease intensity of frosty pod rot in Costa Rica, and mirids in Cameroon. At the individual scale, the number of neighboring cacao trees (at $3.7 \mathrm{~m}$ ) and neighboring fruit trees (at $4.3 \mathrm{~m}$ ) negatively influence the individual intensity of frosty pod rot in Costa Rica. Our results reveal the importance of spatial structure in the description of tropical agroforests and in understanding of the mechanisms influencing the agroecological regulation of pest and diseases within these complex systems. Optimization of spatial tree structure when managing tropical agroforests could be an interesting lever for agroecological control of pests and diseases.
\end{abstract}




\section{Introduction}

\section{Définition du concept de structure spatiale d'une communauté végétale}

La structure spatiale représente l'organisation des individus (plantes) dans l'espace et décrit leurs relations de voisinage. Elle prend en compte les relations spatiales entre les individus (structure horizontale), leur diversité spécifique (composition) et leurs dimensions (structure verticale) (Ngo Bieng, 2013). La structure spatiale détermine les conditions de croissance et la dynamique autour de chacun des arbres du peuplement (nombre et caractéristiques des voisins à plusieurs distances). La structure spatiale rend ainsi compte de la diversité des environnements locaux autour de chacun des individus caractérisant les peuplements hétérogènes. Elle influence ainsi les propriétés naturelles et émergentes des individus dans une communauté. Dans l'étude des mécanismes de régulation agroécologique des bioagresseurs, nous avons appliqué des méthodes d'analyse de la structure spatiale aux systèmes agroforestiers tropicaux à base de cacaoyers. Nous nous sommes intéressés à l'interaction entre la structure spatiale d'une communauté végétale et l'intensité d'attaque de ses bioagresseurs.

\section{Mécanismes d'interaction entre structure spatiale et régulation des bioagresseurs}

La structure spatiale du peuplement végétal associé à la culture agit sur le service de régulation des bioagresseurs à travers la modification du microclimat. En effet, la présence d'arbres associés à une culture tamponne la température de l'air et du sol, diminue la vitesse des vents, ainsi que la quantité et la qualité de lumière transmise. Enfin, elle augmente l'humidité relative de l'air et l'humidité des sols sous canopée (Avelino et al., 2011).

La structure spatiale du peuplement végétal influence ce microclimat à travers ses caractéristiques verticales et horizontales :

- la structure verticale des arbres influence à la fois la quantité et la répartition de la ressource lumineuse sous canopée. En particulier, plus la strate est haute, plus la quantité de lumière transmise sous canopée est faible (Martens et al., 2000), plus le pourcentage d'eau interceptée puis restituée au sol est faible et plus la zone à l'abri du vent est grande;

- la structure horizontale des arbres agit sur la répartition de la ressource lumineuse sous canopée. On observe un ombrage réparti de façon homogène dans les parcelles à structure régulière. Cet ombrage est étalé de façon de plus en plus hétérogène à mesure que la structure des arbres tend vers l'agrégation (Martens et al., 2000).

Les effets directs du microclimat sont nombreux et peuvent se produire à différents moments du cycle de vie des bioagresseurs. Par exemple, la lumière, en particulier les ultraviolets, stimule la sporulation de nombreuses espèces fongiques (Schroth et al., 2000). La réduction du splashing des gouttes de pluie peut diminuer la dispersion des spores (Evans, 1998). Leffet indirect du microclimat sur les bioagresseurs intervient principalement par le biais de son impact sur la culture ou sur les auxiliaires des bioagresseurs (Gidoin et al., 2014b). 


\section{Objectif et cas d'étude}

Lobjectif de ce travail est de démontrer l'influence de l'organisation spatiale des arbres sur l'intensité d'attaque de deux bioagresseurs du cacaoyer dans des agroforêts tropicales. Le cacaoyer est une importante culture pérenne tropicale installée au sein d'agroforêts par la majorité des producteurs des pays du Sud. Ces agroforêts présentent une diversité de structure et de composition. Les deux bioagresseurs étudiés sont les mirides, Sahlbergella singularis, au Cameroun et la moniliose, Moniliophthora roreri, au Costa Rica. Les mirides sont des insectes ailés dont la dissémination est active et peut se faire sur plusieurs kilomètres (Babin et al., 2010). Ils constituent l'une des principales menaces pour la production de cacao en Afrique. La moniliose est un champignon disséminé par le vent sur de grandes distances. Cette maladie fongique est potentiellement la plus dangereuse du cacaoyer. Elle est à l'origine d'importantes pertes pouvant aller jusqu'à $100 \%$ de la production en Amérique tropicale (Gidoin et al., 2014a).

\section{" Matériel et méthodes}

\section{Analyse de la structure spatiale des peuplements et des attaques de bioagresseurs}

Nous présentons l'interaction entre l'organisation spatiale des individus (arbres et cacaoyers) et l'intensité d'attaque des deux bioagresseurs à deux échelles : la parcelle et l'individu (arbres et cacaoyers).

\section{À l'échelle de la parcelle}

Nous avons analysé la structure spatiale des individus (arbres et cacaoyers) dans 36 parcelles de $2000 \mathrm{~m}^{2}$ dans la zone de Talamanca au Costa Rica et dans 20 parcelles de $2500 \mathrm{~m}^{2}$ dans la zone d'Obala au Cameroun. La région de Talamanca est la première zone de production de cacao au Costa Rica. Cette production est assez faible du fait des dommages causés par la moniliose, de l'ancienneté et de la gestion extensive des systèmes, ainsi que de l'éloignement des zones de commercialisation. Les systèmes de culture de la région d'Obala au Cameroun sont plus productifs et gérés plus intensivement. Ils sont situés près de Yaoundé, capitale du pays.

Lanalyse des structures spatiales a été réalisée à partir de la position des individus dans les parcelles. Pour ces analyses, nous avons utilisé la fonction de Ripley, un outil d'analyse de distribution spatiale (Ngo Bieng et al., 2013). Les positions des individus - de hauteur supérieure à $2 \mathrm{~m}$ - dans les parcelles (coordonnées cartésiennes x et y) ont été obtenues à l'aide d'un théodolite (Leica Builder 409, Leica Geosystems, Heerbrugg et Switzerland). Pour chacun des individus positionnés, nous avons noté son espèce et la catégorie à laquelle il appartient : cacaoyer, arbre forestier, fruitier ou bananier. Pour les analyses de la structure spatiale, chaque catégorie a été prise en compte quand elle était représentée par au moins 10 individus dans chaque parcelle étudiée (Ngo Bieng et al., 2013). 
Pour la moniliose, l'intensité d'attaque a été évaluée par le rapport du nombre de cabosses endommagées sur le nombre total de cabosses par parcelle. Quatre relevés par an ont été réalisés en 2008 et 2009 (Gidoin et al., 2014a). Pour les mirides, l'intensité d'attaque a été évaluée par la densité du ravageur par parcelle; un relevé au mois d'août a été réalisé en 2011 et 2012 sur 1600 cacaoyers (Gidoin et al., 2014b).

Nous avons utilisé l'analyse de variance (Anova) pour étudier l'effet de la structure horizontale des arbres forestiers sur la variabilité interparcellaire de l'intensité de la moniliose, d'une part, et de la densité en mirides, d'autre part. Leffet présenté ici n'est pas un artefact du fait de la corrélation de la variable de structure horizontale des arbres forestiers avec une autre variable de structure ou de composition de l'agroforêt, comme le montre l'ensemble des analyses statistiques réalisées sur le même jeu de données. Ces analyses sont présentées en détail par Gidoin et ses coauteurs (Gidoin et al., 2014a; Gidoin et al., 2014b).

\section{À l'échelle de chaque cacaoyer}

Nous avons caractérisé l'environnement local, comme le nombre d'individus voisins sur une distance de de 0 à $10 \mathrm{~m}$, et l'effet de ce voisinage sur l'intensité individuelle d'attaque, c'est-à-dire l'intensité d'attaque de chacun des cacaoyers. Cette analyse a été conduite dans 20 parcelles de $1600 \mathrm{~m}^{2}$ dans la zone d'Upala au Costa Rica. Upala est la deuxième zone de production de cacao après Talamanca. On constate une relance de l'activité cacaoyère avec un ombrage moins dense et moins divers qu'à Talamanca. Elle s'accompagne de la pratique de la récolte sanitaire consistant à retirer des arbres les cabosses endommagées pour évacuer une partie de l'inoculum) et l'utilisation, dans les jeunes plantations, de variétés améliorées partiellement résistantes à la moniliose. Dans les parcelles étudiées, l'intensité individuelle d'attaque par la moniliose a été caractérisée, pour chaque cacaoyer évalué, par le pourcentage de cabosses malades sur le nombre total de cabosses. L'intensité d'attaque individuelle d'un cacaoyer a été expliquée par son nombre de voisins en utilisant un modèle linéaire mixte généralisé (GLMM). En effet, nous cherchons à identifier la catégorie de voisins (cacaoyer, forestier, fruitier et bananier) et la distance de voisinage (0 à $10 \mathrm{~m})$ qui sont corrélées aux plus importants indices d'attaque (Ngo Bieng et al., 2017).

\section{Structure spatiale et distribution de l'ombrage}

Dans cette section, nous nous intéressons à l'étude de l'impact de la structure horizontale et verticale sur la distribution de la ressource lumineuse, via des mesures d'ombrage dans la parcelle. La distribution de la ressource lumineuse est un important élément du microclimat pouvant influencer la dynamique de bioagresseurs. Ici, notre objectif est d'étudier l'effet de la variabilité spatiale de l'ombrage, et donc de la distribution de la ressource lumineuse, sur la distribution spatiale d'un bioagresseur. Cette étude a été réalisée uniquement au Cameroun sur les mirides (insectes photophobiques), dans les 20 parcelles de $2500 \mathrm{~m}^{2}$ installées dans la zone d'Obala et décrites par Gidoin et al. (2014b).

Pour caractériser l'ombrage nous avons utilisé deux méthodes différentes, complémentaires et simples d'utilisation : 
- le logiciel shademotion (version 3, http://shademotion.net) est utilisé pour modéliser l'ombrage. Il permet de calculer $\left(\mathrm{h} / \mathrm{j} / \mathrm{m}^{2}\right)$ l'ombre des arbres associées projetée sur la canopée des cacaoyers. Il calcule la position, la forme de l'ombre projetée par des arbres dans le temps et l'espace en fonction de leurs caractéristiques (hauteur, largeur et densité du houppier, hauteur du tronc et hauteur totale);

- un densiomètre est utilisé pour estimer le taux de couverture totale de la canopée mesurée sous les houppiers des cacaoyers. Les mesures de densiomètre ont été prises dans les parcelles dans un format de $5 \times 10 \mathrm{~m}$, ce qui donne 66 mesures par parcelle. Linterpolation pour arriver à une valeur par $\mathrm{m}^{2}$ a été effectuée par krigeage en utilisant le logiciel GS+ (http://www.gammadesign.com/).

\section{W Résultats}

\section{Relation entre structure spatiale et régulation des bioagresseurs}

\section{À l'échelle de la parcelle}

Nos résultats montrent que la structure spatiale horizontale des arbres forestiers a un effet significatif sur la moniliose au Costa Rica (fig. 7.1a) et sur les mirides au Cameroun (fig. 7.1b).

Au Costa Rica, l'intensité d'attaque de la moniliose est plus élevée dans les parcelles caractérisées par une faible densité en arbres forestiers et une structure agrégée de ces arbres (i.e. des arbres répartis en agrégats dans les parcelles). Elle est plus basse dans les parcelles à structure aléatoire et les parcelles à structure régulière des arbres forestiers (Gidoin et al., 2014a).

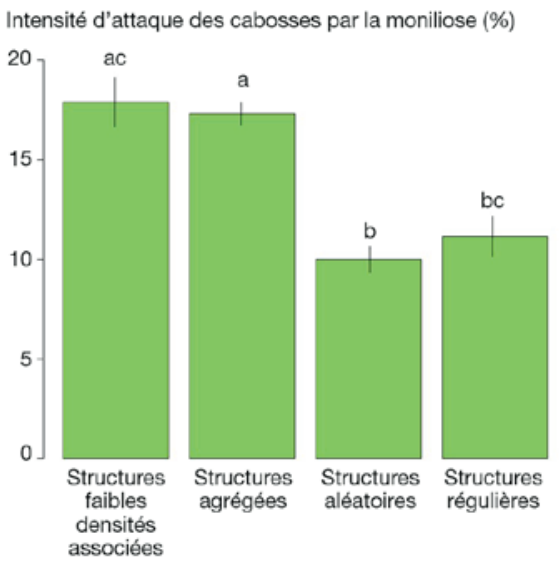

Figure 7.1a. Intensité d'attaque des cabosses de cacaoyer par la moniliose.

Anova : $\mathrm{F}=5,26$ pvalue $<0,01$

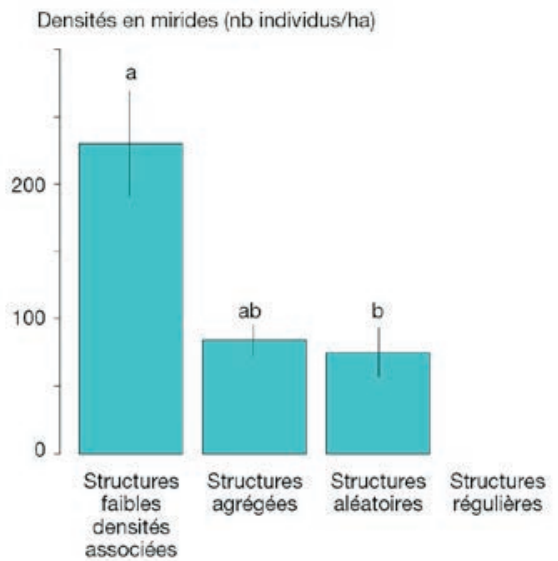

Figure 7.1b. Densité de mirides en fonction des types de structures des arbres associés.

Anova : $\mathrm{F}=3,88$ pvalue $<0,05$. Les moyennes présentées avec des lettres différentes sont significativement différentes (test de Tukey HSD), les barres d'erreurs représentent l'erreur standard. 
Au Cameroun, la densité de mirides décroît lorsqu'un nombre minimal d'arbres forestiers est aléatoirement réparti (i.e distribué au hasard) dans la parcelle (75 insectes/ ha en moyenne en 2011 et 2012) en comparaison à des parcelles ayant une structure agrégée d'arbres forestiers (84 insectes/ha) ou à faible densité en arbres forestiers (230 insectes/ha) (Gidoin et al., 2014b).

\section{À l'échelle du cacaoyer, au Costa Rica}

Dans notre jeu de données, nous avons rencontré une variabilité individuelle de l'intensité d'attaque de la moniliose, entre 0 et $100 \%$ de cabosses malades par cacaoyer. À la suite de l'exploration des variables, il ressort que le nombre des cacaoyers (nca) dans un rayon de 3,7 m (nca3.7) et le nombre de fruitiers (nfu) dans un rayon de 4,3 m (nfu4.3) sont les variables de l'environnement local expliquant le mieux la variabilité individuelle de l'intensité d'attaque. Dans les deux cas, l'interaction est négative : plus il y a de voisins cacaoyers à $3,7 \mathrm{~m}$ ou de fruitiers à 4,3 $\mathrm{m}$, moins l'intensité d'attaque individuelle est importante (fig. 7.2). Le nombre de voisins cacaoyers et le nombre de fruitiers à 4,3 m expliquent respectivement environ $15 \%$ et $7 \%$ de la variabilité de l'intensité individuelle de la moniliose (fig. 7.2). La prédiction de l'intensité d'attaque est aux alentours de $20 \%$ pour un cacaoyer avec un nca3.7 = 1 et de $2,5 \%$ pour un cacaoyer avec un nca3.7 $=8$ (fig. $7.2 \mathrm{a}$ ). Elle est aux alentours de $10 \%$ pour un cacaoyer ayant un nfu $4.3=0$ et de $1 \%$ pour un cacaoyer ayant un nfu4.3 = 3 (fig. 7.2b). Les nombres d'arbres forestiers et de bananiers dans l'environnement local n'ont pas d'influence sur l'intensité d'attaque des individus.

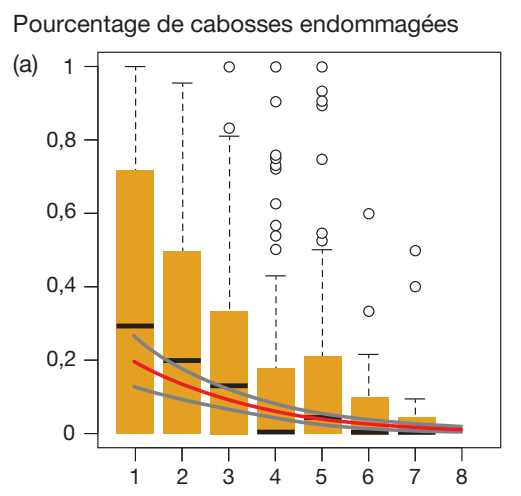

Nombre de cacaoyers dans un rayon de $3,7 \mathrm{~m}$
Pourcentage de cabosses endommagées

(b)

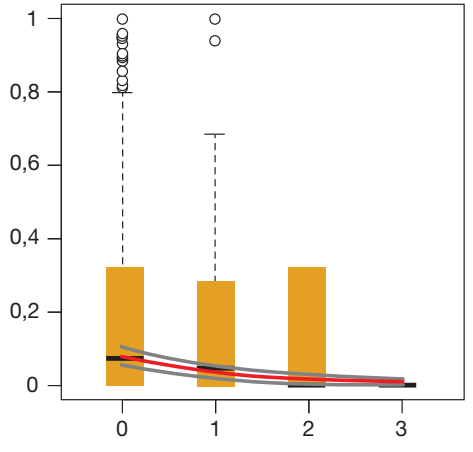

Nombre de fruitiers dans un rayon de $3,7 \mathrm{~m}$

Distribution de la variabilité individuelle

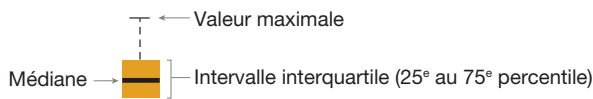

- Prédiction moyenne de l'intensité individuelle

- Écart-type

Figure 7.2a. Mesures (boîtes à moustaches) et prédictions par le GLMM (courbes) de l'intensité d'attaque individuelle des cacaoyers par la moniliose en fonction du nombre de cacaoyers situés à 3,7 m (nca3.7) (a) ou du nombre de fruitiers situés à 4,3 m (nfu4.3) (b). nca3.7 explique environ $15 \%$ de la variabilité de l'intensité d'attaque individuelle. nfu4.3 explique environ $7 \%$ de la variabilité de l'intensité d'attaque individuelle. 


\section{Structure spatiale, distribution de l'ombrage et impact sur les mirides au du Cameroun}

En utilisant le logiciel Shademotion et en comparant les parcelles de même composition et densité d'arbres d'ombrage, nous observons qu'une structure agrégée entraîne une hétérogénéité et une variance plus importante du taux d'ombrage qu'une structure aléatoire. À structure spatiale et à densité fixes, la composition a un impact sur la distribution de l'ombrage à travers l'architecture des peuplements étudiés. Des analyses de semi-variance montrent la dépendance spatiale de la distribution des mirides au sein des parcelles avec une structure agrégée des arbres d'ombrage. Les mirides ont tendance à se localiser dans les zones moins ombragées au sein des parcelles à structure agrégée (fig. 7.3a et fig. 7.3b). Cette dépendance spatiale n'est pas observée dans les parcelles à structure aléatoire. En utilisant les données du densiomètre, nous observons que ces zones moins ombragées par les arbres associés ont des valeurs élevées de couverture totale de la canopée (arbres associés + cacaoyers) (fig. 7.3c). Ceci pourrait être lié aux observations qui montrent que la densité de mirides est liée à la quantité de tissus sensibles (Gidoin et al., 2014b). Des cacaoyers situés dans des zones moins ombragées reçoivent plus de ressource lumineuse. Lintensité lumineuse plus élevée permet une croissance plus vigoureuse des cacaoyers, ce qui peut se traduire par:

- plus de ressources alimentaires (cabosses et jeunes pousses) pour les mirides;

- un taux de couverture totale arborée plus élevé expliqué en partie par un niveau d'auto-ombrage des cacaoyers plus élevé (fig. 7.3c).

\section{"Discussion}

Nos résultats mettent en évidence une diversité d'organisations spatiales des individus dans les systèmes agroforestiers à base de cacaoyers étudiés au Costa Rica et au Cameroun. De plus, ils montrent une influence significative de l'organisation spatiale des individus sur les intensités d'attaque de deux bioagresseurs du cacaoyer dans des agroforêts au Costa Rica et au Cameroun. Cette influence significative se fait par le biais d'une modification de l'environnement local autour de chacun des individus hôtes, les cacaoyers. Cette modification de l'environnement local implique un changement des conditions microclimatiques autour des cacaoyers. Et ce sont principalement des effets microclimatiques en relation avec les conditions de développement épidémiologiques des bioagresseurs étudiés que nous discutons ici.

À l'échelle de la parcelle, nous montrons que des organisations spatiales régulières sont corrélées à de plus faibles intensités d'attaques de la moniliose au Costa Rica (Talamanca) et des mirides au Cameroun (Obala). On observe la même tendance pour des organisations spatiales alétoires d'arbres forestiers.

Cas de la moniliose. La moniliose est une maladie fongique dont les conditions microclimatiques de plein soleil facilitent la dispersion (faible humidité relative de l'air et vitesse des vents importante), tandis que les conditions sous ombrage (forte humidité relative de l'air) facilitent le développement des spores. Ces deux condi- 


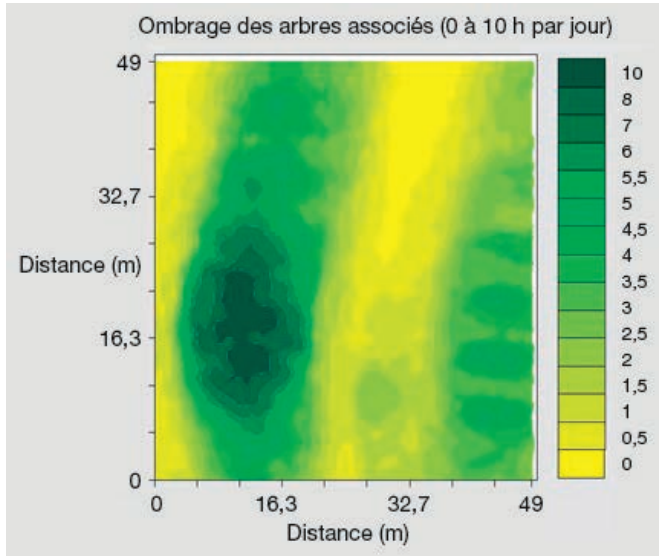

Figure 7.3a. Distribution de l'ombrage journalier (en heures par jour) dans une parcelle avec une structure agrégée des arbres associés.

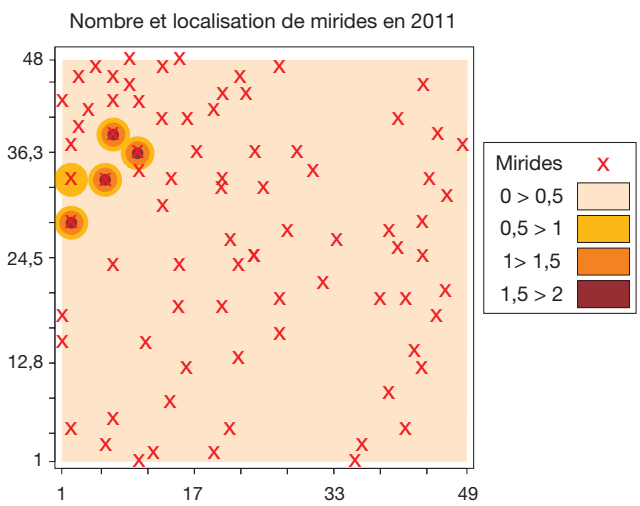

Figure 7.3b. Distribution et nombre de mirides et positions des cacaoyers échantillonnés dans une parcelle avec une structure agrégée des arbres associés.

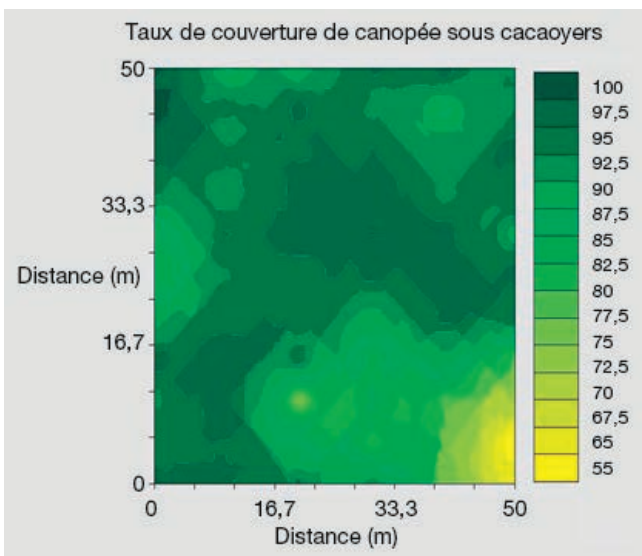

Figure 7.3c. Taux de fermeture de canopée en pourcentage dans une parcelle avec une structure agrégée des arbres associés. 
tions sont rencontrées dans les parcelles à faible densité en arbres forestiers et à structure agrégée des arbres forestiers, respectivement entre les agrégats et sous les agrégats d'arbre forestiers; ce qui pourrait expliquer les forts taux d'attaque de moniliose observés dans ces parcelles (Gidoin et al., 2014a).

Cas des mirides. Dans les parcelles à structure agrégée des arbres forestiers, la densité plus élevée de mirides pourrait être expliquée par leur présence dans des zones éclairées (entre les agrégats d'arbres forestiers). En effet ces zones pourraient fournir plus de ressources (cabosses et jeunes pousses de cacaoyers) à ces insectes piqueurs suceurs. Dans ces zones moins ombragées, les conditions de développement des mirides sont cependant considérées comme sous-optimales, les mirides étant des insectes photophobiques (Babin et al., 2010). Laugmentation de l'autoombrage (ombrage donné par le cacaoyer lui-même) pourrait alors compenser les effets négatifs du manque d'ombrage entre les agrégats sur le développement des mirides. En effet, ces zones moins ombragées correspondent à des houppiers de cacaoyer plus grands et plus denses, comme en témoignent les mesures de fermeture plus élevées dans ces zones (fig. 7.3c, valeurs de densiomètre prise en dessous du canopée des cacaoyers).

Dans des systèmes plein soleil caractérisés par une abondance de ressources, l'agrégation spatiale des mirides semble moins stable dans le temps, et leurs dégâts distribués plus uniformément (Mahob et al., 2015). Néanmoins, les travaux portant sur l'interaction entre organisation spatiale des arbres, distribution de l'ombrage et les mirides ont, pour l'instant, été menés sur un nombre limité de parcelles. Il serait intéressant de les réaliser sur un plus grand nombre de dispositifs.

Les variables de structure spatiale. Il est intéressant de noter que les variables de structure spatiale ayant des effets significatifs dans notre étude sont rarement prises en compte à l'échelle de la parcelle dans la littérature. En effet, dans la majorité des études s'intéressant à la formation d'un microclimat au sein des systèmes agroforestiers, le microclimat est décrit à l'aide de mesures directes (mesures photo-hémisphériques par exemple) ou à partir des variables de structure des arbres d'ombrage, généralement la densité en arbres d'ombrage. Nos résultats révèlent l'importance de variables de structure spatiale. Ils donnent des pistes nouvelles pour la description des systèmes complexes et l'étude des mécanismes influençant leur fonctionnement.

Variabilité individuelle de l'intensité d'attaque de la moniliose. À l'échelle des cacaoyers, nous avons mis en évidence une variabilité individuelle de l'intensité d'attaque de la moniliose. Le nombre d'individus autour des cacaoyers étudiés explique une partie de cette variabilité. En effet, le nombre de cacaoyers dans un rayon de 3,7 $\mathrm{m}$ a une influence négative sur l'intensité d'attaque de moniliose. Nous faisons l'hypothèse que cette influence est due à un effet barrière dans la dispersion des pathogènes lié à l'architecture du cacaoyer (Ngo Bieng et al., 2017). Des traits architecturaux comme le LAI, la hauteur du houppier, la rugosité du houppier, la longueur des branches, la position des branches et des feuilles réduisent la porosité du houppier des cacaoyers (i.e. pourcentage de vides présents dans le houppier), conduisant à des distances de dispersion réduites. De plus, les branches de cacaoyers peuvent s'étaler du sommet jusqu'au sol, ce qui contribue à renforcer la porosité du houppier. Ces traits architecturaux sont pertinents en 
cas de dispersion passive de spores : c'est le cas des maladies fongiques telles que la moniliose. Les organes des cacaoyers non hôtes de la maladie constituent une barrière efficace à la dispersion des spores de la moniliose jusqu'aux organes hôtes, les cabosses. Une distribution spatiale optimale des organes non hôtes aux alentours de chacun des cacaoyers infectés participe ainsi à une réduction de la dispersion de la maladie.

Concernant les arbres forestiers et fruitiers des strates supérieures, en plus de leur faible densité, leur architecture contribue peu à leur porosité, car seuls leurs troncs agissent comme barrières à la distribution des spores. En plus de cet effet barrière limité, les fruitiers ont aussi un impact sur la disponibilité de la lumière autour du cacaoyer considéré. En taille, les fruitiers sont plus petits que les arbres forestiers et fournissent un ombrage plus dense et plus localisé. Cet ombrage localisé réduit ainsi la production des cacaoyers ayant des fruitiers dans leur voisinage. D'ailleurs, nous avons observé que plus un cacaoyer a de voisins fruitiers dans un rayon de 4,3 m, moins il est infecté par la moniliose.

Importance du choix des arbres d'ombrage et de leur organisation spatiale. Nos résultats permettent de souligner l'importance du choix des arbres d'ombrage et de leur organisation spatiale dans les systèmes agroforestiers. Au Cameroun par exemple, l'intensification des systèmes agroforestiers se traduit par une augmentation de la densité en fruitiers et en arbres à produits forestiers non ligneux. Cependant, les arbres forestiers constituant la canopée de ces systèmes présentent des houppiers plus larges et moins denses que les fruitiers (Gidoin et al., 2014a). Les arbres forestiers fournissent en plus un grand nombre de services écosystémiques dans les parcelles agroforestières. De par leur architecture, ils fournissent un ombrage homogène quand ils sont régulièrement ou aléatoirement répartis, ombrage nécessaire à la durabilité de la production du cacaoyer.

\section{W Conclusion}

Dans ce travail, nous montrons que l'organisation spatiale des individus, rarement décrite dans la littérature, influence l'intensité d'attaque de bioagresseurs. Nos analyses indiquent que des organisations spatiales régulières d'arbres forestiers sont corrélées à de plus faibles intensités d'attaques de la moniliose et des mirides du cacaoyer. Il en est de même pour des organisations spatiales aléatoires, elles aussi corrélées à de plus faibles intensités d'attaques des deux bioagresseurs étudiés. Plus spécifiquement dans le cas de la moniliose et dans des parcelles présentant de telles organisations spatiales n'exacerbant pas le développement de la moniliose, nous mettons en évidence un effet barrière des cacaoyers sur la dispersion des spores.

Nos résultats participent à la mise en place de régulation agroécologique des bioagresseurs dans les systèmes agroforestiers, par le biais de l'optimisation de l'organisation spatiale des individus cultivés, dans l'objectif de limiter la réceptivité des parcelles agroforestières aux insectes ravageurs et agents pathogènes. La gestion de la structure spatiale (horizontale) des arbres d'ombrage pourrait ainsi constituer un levier intéressant pour la gestion agroécologique des bioagresseurs. 


\section{- Bibliographie}

Avelino J., Ten Hoopen G.M., Declerck F., 2011. Ecological mechanisms for pest and disease control in coffee and cacao agroecosystems of the Neotropics. In: Rapidel B., DeClerck F., Le Coq J.-F., Beer J., (eds). Ecosystems services from agriculture and agroforestry. Londres, Royaume-Uni: Earthscan, p. 320.

Babin R., Hoopen G.M.T., Cilas C., Enjalric F., Yede G.P., Lumaret J.P., 2010. Impact of shade on the spatial distribution of Sahlbergella singularis in traditional cocoa agroforests. Agricultural and forest entomology, 12: 69-79.

Gidoin C., Avelino J., Deheuvels O., Cilas C., Ngo Bieng M.N., 2014a. Shade tree spatial structure and pod production explain frosty pod rot intensity in cacao agroforests, Costa Rica. Phytopathology, 104(3): 275-281.

Gidoin C., Babin R., Beilhe L.B., Cilas C., Hoopen G.M.T., Ngo Bieng M.N., 2014b. Tree spatial structure, host composition and resource availability influence mirid density or black pod prevalence in cacao agroforests in cameroon. PLoS one, 9 (10).

Mahob R., Baleba L., Yédé, Dibog L., Cilas C., Bilong Bilong C.F., Babin R., 2015. Spatial distribution of Sahlbergella singularis Hagl. (Hemiptera: Miridae) populations and their damage in unshaded young cacao-based agroforestry systems. International Journal of Plant, Animal and environmental sciences, 5(2): 121-131.

Martens S.N., Breshears D.D., Meyer C.W., 2000. Spatial distributions of understory light along the grassland/forest continuum: effects of cover, height, and spatial pattern of tree canopies. Ecological modelling, 126(1): 79-93.

Ngo Bieng M.A., Alem L., Curtet C., Tixier P., 2017. Tree spacing impacts the individual incidence of Moniliophthora roreri disease in cacao agroforests. Pest management science, 10.1002/ps.4635, n/a-n/a.

Ngo Bieng M.A., Gidoin C., Avelino J., Cilas C., Deheuvels O., Wery J., 2013. Diversity and spatial clustering of shade trees affect cacao yield and pathogen pressure in Costa Rican agroforests. Basic and applied ecology, 14(4): 329-336.

Schroth G., Krauss U., Gasparotto L., Duarte-Aguilar J.A., K.V., 2000. Pests and diseases in agroforestry systems of the tropics. Agroforestry systems, 50: 199-241.

Watve M.G., Jog M.M., 1997. Epidemic diseases and host clustering: An optimum cluster size ensures maximum survival. Journal of theoretical biology, 184(2): 167-171. 

Partie 2

Les parcs agroforestiers arbores et arbustifs à base de cultures annuelles en Afrique de l'ouest 

A - Les parcs arborés 



\title{
Chapitre 8 \\ La crise du parc arboré à karité : exemple de Djougou au Bénin
}

\author{
Bidou J.-É., Koukpéré A. et Droy I.
}

Résumé. Les parcs arborés à dominante de karité s'étendent sur une bande de l'Afrique soudanienne du Sénégal à l'Éthiopie. Ils sont le résultat d'un système agraire associant jachère et culture, dans lequel certains arbres sont préservés et entretenus en raison de leurs fonctions nourricières, notamment l'apport en matière grasse par les noix de karité. Or, malgré une demande importante en noix de karité sur le marché international, beaucoup de ces parcs sont en voie de dégradation, comme à Djougou au Bénin. Afin de mieux comprendre cette situation paradoxale, cette étude présente la complexité des dynamiques en cours en liant, sur plusieurs échelles, les évolutions du système agroforestier aux changements sociaux, en particulier aux inégalités entre femmes et hommes sur la gestion de la ressource arborée.

Abstract. Shea trees parklands spread across sudanian Africa from Senegal to Ethiopia. They are the outcome of a farming system marked by long fallows where some trees were spared and even maintained for their food supply, like shea nut trees for vegetable fat. Yet, in spite of a strong demand of shea nut butter on the international market, many of these parklands are deteriorating, like in the case of Djougou, in Benin. For a better understanding of this paradoxical situation, this study presents the complexity of the ongoing processes, linking at different scales the evolution of the agroforestry system and social change, in particular gender inequality in wooded resource management.

\section{Introduction}

Depuis la fin des années 1970, les parcs arborés africains, dont les plus emblématiques sont les parcs à karité (Vitellaria paradoxa), connaissent de profondes évolutions entre disparition, dégradation, maintien, extension ou plus rarement régénération (Boffa, 2000). Ces situations contrastées reflètent l'évolution des modes d'exploi- 
tation et de régulation de ces espaces. En effet, il a été montré que les paysages de parcs arborés, au-delà des besoins matériels d'un groupe humain, révélaient également son histoire, sa structure sociale et son organisation (Pélissier, 1980). On trouve ainsi une bande de parcs arborés à karité qui couvre toute l'Afrique soudanienne du Sénégal à l'Éthiopie.

\section{Encadré 8.1 - Les parcs arborés d'Afrique de l'Ouest}

Les parcs arborés d'Afrique de l'Ouest sont des formations végétales que les biogéographes qualifient de savanes arborées ou boisées. Pour les agronomes, ce sont des parcs agroforestiers, désignant ainsi un mode d'utilisation des terres dans lequel certains arbres (comme les karités ou les nérés) sont préservés des défrichements pour leurs fonctions d'approvisionnement et intégrés dans un système de production associant le plus souvent d'autres cultures (céréales, tubercules ou autres plantations).

Pendant longtemps, la densité de peuplement de karités a accompagné les densités humaines; les populations construisant les parcs arborés tout au long des cycles de jachères et de défrichement dans le cadre d'une agriculture sur brûlis. Les noix germent et les jeunes plants de karités se développent dans les jachères. Lors du défrichement par brûlis, les pieds les plus prometteurs sont épargnés; ils peuvent désormais grandir et grossir dans les champs sans grande concurrence, jusqu'à la prochaine jachère. Si ces arbres sont protégés, éclaircis, sélectionnés et entretenus tout au long de leur vie, souvent longue de plus de deux cents ans, ils ne sont cependant pas plantés comme dans un verger (Agbahungba et Depommier, 1989; Serpantié, 1996a et 1996b; Serpantié et al., 1996; Ouédraogo et Devineau, 1997; Lovett et Haq, 2000).

Depuis une vingtaine d'années, les parcs à karités ${ }^{1}$ ont suscité un regain d'intérêt pour la recherche dans trois domaines principaux :

- reconnu comme une des espèces principales des jachères nourricières, le karité a suscité de nombreuses études en biologie, botanique, ethnobotanique et agronomie (Kakaï et al., 2011);

- présenté, à tort ou à raison, comme «l'or blanc des femmes», il a suscité des travaux liant le genre, le pouvoir d'agir des femmes et la lutte contre la pauvreté (Saussey et al., 2008; Pouliot, 2012; Pouliot et Elias, 2013);

- la chaîne de valeur du karité et, d’une façon plus générale, le contexte socio-politique de la filière constituent le troisième domaine de recherche (Rongead, 2014; Rousseau et al., 2015).

Au Bénin, ces parcs à karités sont plutôt en voie de dégradation, alors que la demande en noix et en beurre de karité s'est fortement accrue sur le marché international depuis quatre décennies et que, parallèlement, le monde prend conscience du rôle de l'arbre dans le cadre d'une agriculture durable (Garrity et al., 2010). Alors que de nombreux projets ont pour objectif l'amélioration des filières commerciales par le soutien aux groupements de femmes collectrices et transformatrices du karité,

1. Par simplification, les parcs arborés où domine le karité sont appelés «parc à karité », mais ils comptent aussi d'autres essences forestières comme le néré (Parka biglobosa). 
ceux-ci disparaissent pour des raisons variées : surtout faute de régénération, car il n'y a pas ou peu de jeunes arbres pour remplacer ceux qui sont éliminés par abattage, maladie ou vieillissement (Bidou et al., 2018).

Le dysfonctionnement apparent de ce système agroforestier est donc paradoxal et révèle la complexité des dynamiques à l'œuvre dans l'évolution des parcs arborés. Il nécessite de prendre en compte l'articulation de plusieurs échelles, en liant les évolutions du système agroforestier aux évolutions sociétales.

\section{W Articulation d'échelles et de méthodes}

Dans une étude conduite à Djougou dans le Nord-Est du Bénin (fig. 8.1), la dynamique du parc agroforestier est mise en relation avec la place du karité dans les revenus des femmes et l'évolution de la filière béninoise du karité. Pour réaliser cette articulation d'échelles, qui va du champ à la région, nous avons mis en relation des enquêtes socio-économiques auprès des ménages ruraux, notamment des femmes, avec des analyses d'images prises par satellite.

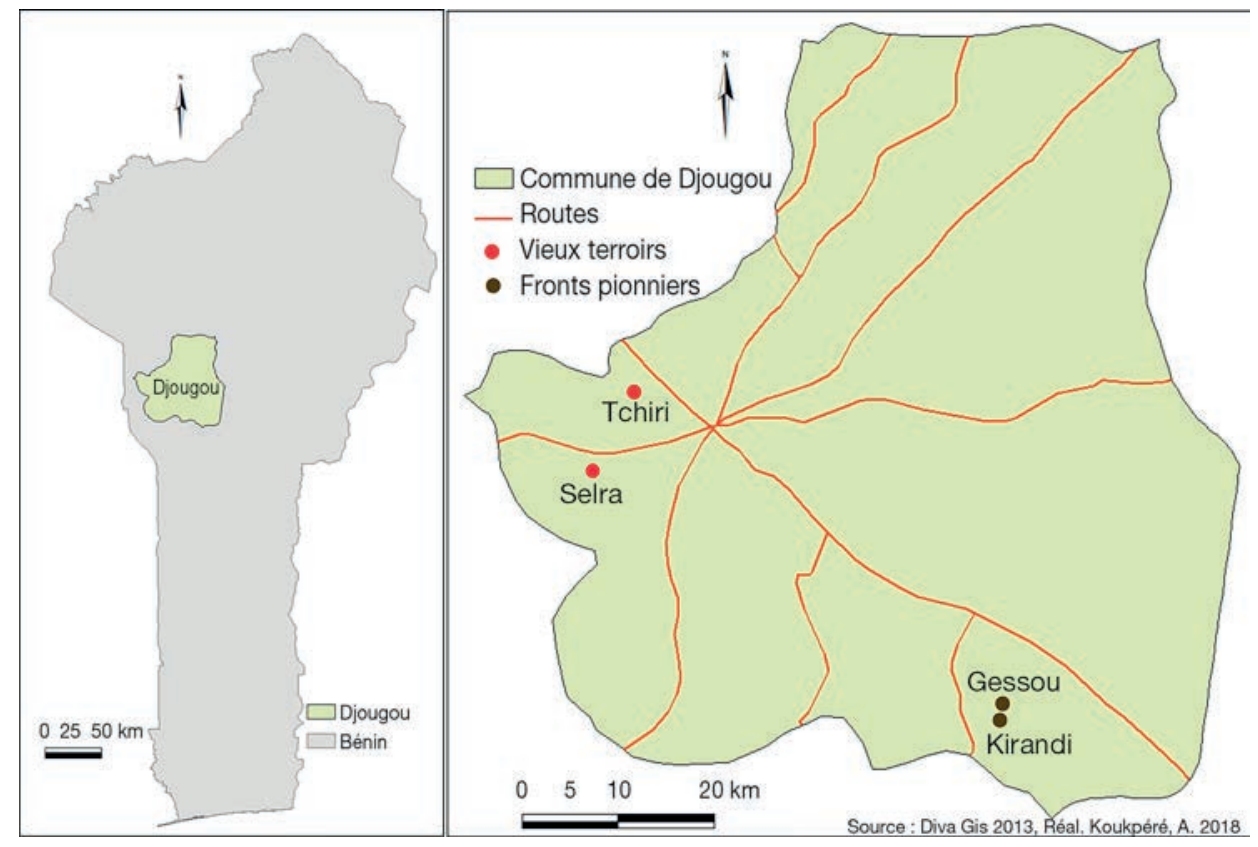

Figure 8.1. Localisation de la commune de Djougou au Bénin et des sites d'enquêtes.

\section{Dispositif d'enquêtes à l'échelle des ménages et des champs}

À Djougou, comme dans tout le Nord du Bénin, la population s'organise autour de vieilles chefferies ou de petits royaumes. Les terroirs anciens proches de la ville sont occupés depuis au moins le XVII ${ }^{\mathrm{e}}$ siècle. La localisation des villages y est stabilisée depuis longtemps, et le paysage est celui d'un parc à karités avec des densités de population 
qui dépassent actuellement 50 hab./ $\mathrm{km}^{2}$. À une trentaine de kilomètres de la ville, l'est et le sud de la commune sont encore largement occupés par des forêts sèches en cours de défrichement depuis plusieurs décennies. Les tenures foncières y sont encore mal stabilisées autour des villages récents dont la population s'accroît rapidement. Mais les densités de population demeurent relativement faibles (10 à $\left.15 \mathrm{hab} / \mathrm{km}^{2}\right)$. Les villages essaiment à partir des hameaux de culture qui deviennent permanents. Sur ces fronts pionniers, l'accessibilité des villages est très inégale avec des conséquences sur les activités pratiquées et sur les conditions de vie (Bidou et al., 2018).

Cette étude est issue des résultats d'enquêtes menés dans le cadre du projet Safse, volet «Les conditions sociales et économiques de gestion des parcs à karité au Bénin : maintien ou dégradation d'un système?». Nous avons aussi mobilisé les données d'un projet précédent sur lequel nous avions fait des enquêtes en 2011 sur la même zone (ANR-ECliS, Élevage-climat-société).

Dans la commune de Djougou, diverses enquêtes socio-économiques liées à des projets de recherche successifs ont permis d'établir une base de données qui s'étoffe au cours des années. À l'enquête socio-économique auprès des ménages de 2010, se sont succédées d'autres enquêtes en 2014 et 2015, dont celles sur les parcs arborés. Pour cette dernière, nous avons choisi un échantillon de 40 ménages à partir de l'enquête socioéconomique de 2010 auxquels nous avons distribué deux formulaires. Le premier formulaire a permis de collecter les informations sur les champs : la position du champ principal dans le finage, son organisation, l'historique des cultures et des jachères, les méthodes de défrichement et l'exploitation du karité. Le deuxième formulaire a permis de collecter des informations sur les arbres : position dans les champs étudiés, les espèces et les caractéristiques morphométriques des arbres adultes. Le comptage des karités a été fait à partir d'arbres ayant une taille de plus de 1,30 m. En effet, un karité de 1,30 m est âgé de quelques années, il a donc été préservé ou a survécu aux éventuels brûlis. Les nombreuses plantules n'ont pas été prises en compte.

Une enquête de terrain a eu lieu de juillet à septembre 2014, dans quatre localités de la commune de Djougou. Deux des localités sont situées dans les terroirs anciens, à moins de $15 \mathrm{~km}$ du chef-lieu de la commune Djougou, au nord-ouest (Tchiri) et au sud-ouest (Selra). Les deux autres localités sont situées sur le front pionnier (Guessou et Kirandi), à $40 \mathrm{~km}$ environ au sud-est du chef-lieu de commune (fig. 8.1). Au cours de l'enquête, 40 exploitations possédant des champs cultivés et des jachères ont été retenues. Au total, les enquêtes menées dans 115 champs et 35 jachères ont permis de collecter beaucoup d'informations.

\section{Les images satellitaires}

En première approximation, il est possible de recourir à la télédétection pour suivre et analyser les changements d'occupation du sol et mettre ainsi en évidence les variations de la superficie du parc agroforestier. Dans la commune de Djougou, cette évolution a été étudiée à partir de trois cartes d'occupation du sol en 1972, 1990 et 2000 réalisées à partir de scènes Landsat. La résolution spatiale des scènes utilisées ne permet pas de détecter les arbres isolés, ni les petits groupements d'arbres et encore moins d'en déterminer l'espèce. Mais on retrouve, par classification multispectrale, les différents types d'occupation du sol en fonction de la densité du couvert 
végétal (Andrieu et Mering, 2008). Les surfaces de sols nus ont été interprétées comme étant des espaces cultivés en permanence (champs). Les savanes boisées ayant une végétation ouverte sur sol clair (i.e. taux de recouvrement de moins de $30 \%$ ) sont considérées comme des jeunes jachères courtes. Dans le même esprit, on a assimilé à une jachère longue les surfaces de savanes boisées où le recouvrement est supérieur à $30 \%$, sans atteindre celui de la forêt. De fait, il y a là divers stades de recrû forestier après culture, typiques de l'évolution d'un parc agroforestier dans un système de défriche-brûlis.

Les images satellitaires renseignent sur l'évolution du parc, pas sur leur composition floristique. Or, chaque parc est modelé en fonction des conditions agroécologiques d'adaptation des arbres, mais aussi au choix des communautés villageoises conformément à leurs besoins et leurs traditions (Walla et al., 2005). Les parcs soudaniens s'organisent autour d'un certain nombre d'espèces comme le karité (Vitellaria paradoxa) et le néré (Parkia biglobosa), que l'on trouve à Djougou. D'autres parcs sont à dominante de Faidherbia albida, d'Adansonia digitata (baobab) ou de Borassus aethiopum (rôniers). Parallèlement, ces parcs évoluent au cours du temps pour s'adapter aux besoins des populations.

\section{Dynamique des parcs agroforestiers dans la région de Djougou}

\section{Évolutions des terroirs anciens et des fronts pionniers}

Lanalyse des images d'occupation du sol (recouvrement arboré) montre une forte régression des formations arborées dans les terroirs anciens, comparés aux villages des fronts pionniers. Dans les terroirs anciens, la forêt a depuis longtemps disparu ou ne présente que des reliques. Les vieilles jachères (de plus de dix ans) couvrent moins de $10 \%$ du finage en 2000. L'essentiel des terroirs se distribue entre des jachères courtes (d'un à trois ans) et des champs cultivés. Beaucoup de champs sont désormais sans végétation ligneuse (sols nus).

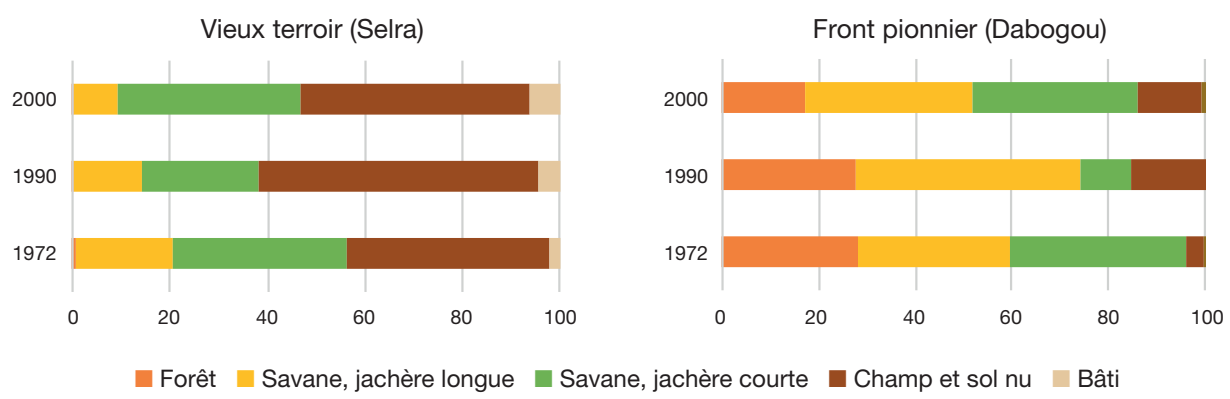

Figure 8.2. Évolution de l'occupation du sol des anciens terroirs et des fronts pionniers entre 1972 et 2000.

La typologie des terroirs est réalisée en fonction du taux de recouvrement (densité du couvert végétal) : forêt; savane boisée, jachère longue; savane boisée, jachère courte, champ et sol nu; bâti (source : images Landsat 1972, 1990 et 2000). 
Dans les fronts pionniers proches de Guessou et Kirandi, on constate un recul de la forêt entre les années 1970 et 2000, tandis que les surfaces cultivées progressent, mais à un rythme beaucoup plus lent. Ce sont surtout les jachères courtes et longues qui s'étendent.

\section{Une régénération insuffisante}

Dans ces parcs à la composition floristique diversifiée, la densité de peuplement en karités varie beaucoup en fonction des facteurs écologiques, mais aussi, de façon générale, de leur distance au village ${ }^{2}$. Dans les champs proches des villages de Guessou et Kirandi, dans les fronts pionniers, les arbres sont aussi peu nombreux que dans les anciens terroirs à Selra et Tchiri : on compte une dizaine d'arbres par hectare, qui sont des individus de grande taille et déjà âgés. En revanche, lorsque l'on s'écarte du village, le nombre de pieds s'accroît dans les fronts pionniers, jusqu'à une trentaine par hectare et quelquefois beaucoup plus (fig. 8.3), alors qu'il reste stable dans les anciens terroirs. À partir d'une soixantaine de pieds par hectare laissés lors du défrichement de la forêt, on élimine au fur et à mesure les moins productifs et les moins bien placés, car, en grandissant, les arbres font de l'ombre aux cultures qui restent l'élément essentiel de l'agrosystème forestier. Bien qu'en principe, les cycles de culture et de jachère permettent à de nouvelles générations d'arbres de rejoindre celle issue du premier défrichement, la régénération naturelle est de moins en moins facile avec le raccourcissement de la durée des jachères dans des espaces de plus en plus peuplés. C'est ce qui explique les faibles densités de karités déjà très âgés dans les anciens terroirs.

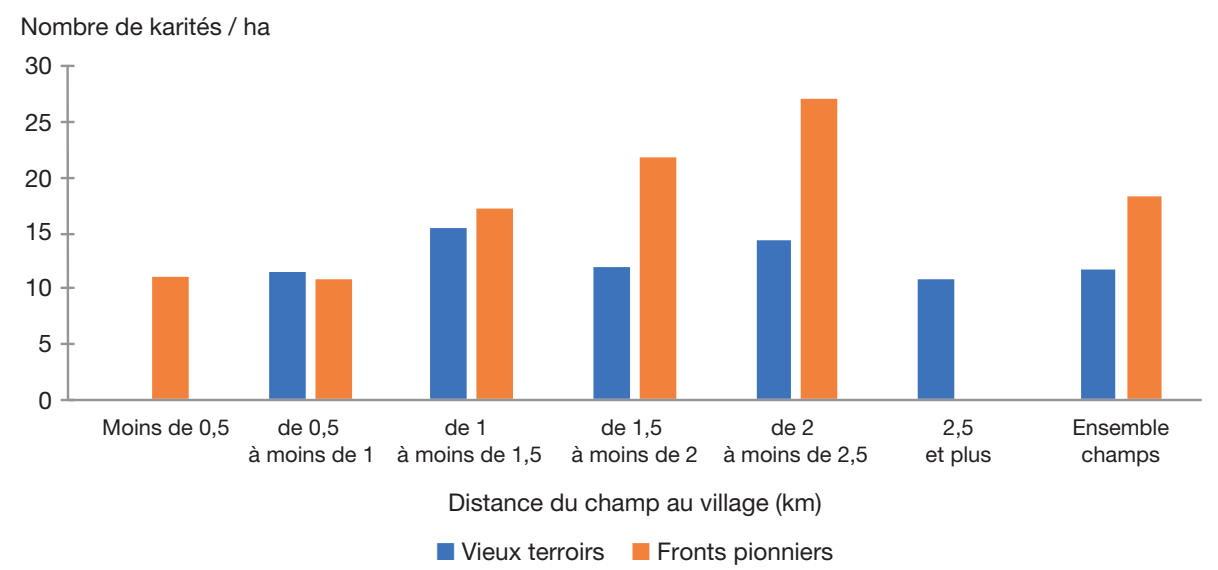

Figure 8.3. Densité de peuplement de karités par hectare dans les champs selon la distance au village $(\mathrm{km})$.

Source : enquête des auteurs, 2014. Anciens terroirs : villages de Selra et Tchiri. Fronts pionniers : villages de Guessou et Kirandi. Labsence de données montre qu'il n'y a pas eu d'observation en raison de la taille de l'échantillon. Le nombre de champs inventoriés (40) correspond au principal champ du ménage ayant l'objet d'un relevé systématique des ligneux.

2. Ce phénomène a été très bien décrit au Burkina Faso : «le karité s'épanouit puis s'éloigne, avec l'expansion des champs et de l'habitat» (Ouédraogo et Devineau, 1997). 
Pourtant, au moins dans une grande partie de la commune de Djougou, la composition arborée des jachères montre que la régénération du karité ne devrait pas rencontrer de grandes difficultés. Sur les fronts pionniers, les deux tiers des champs ont été défrichés sur des recrûs forestiers de plus de 15 ans. Seule une minorité de champs près des villages ont été ouverts après des jachères assez courtes; non par manque de terre, mais pour des raisons pratiques de proximité. La situation est plus tendue dans les anciens terroirs où les jachères courtes prévalent, avec un tiers des champs cultivés après des jachères de moins de 5 ans (fig. 8.4).
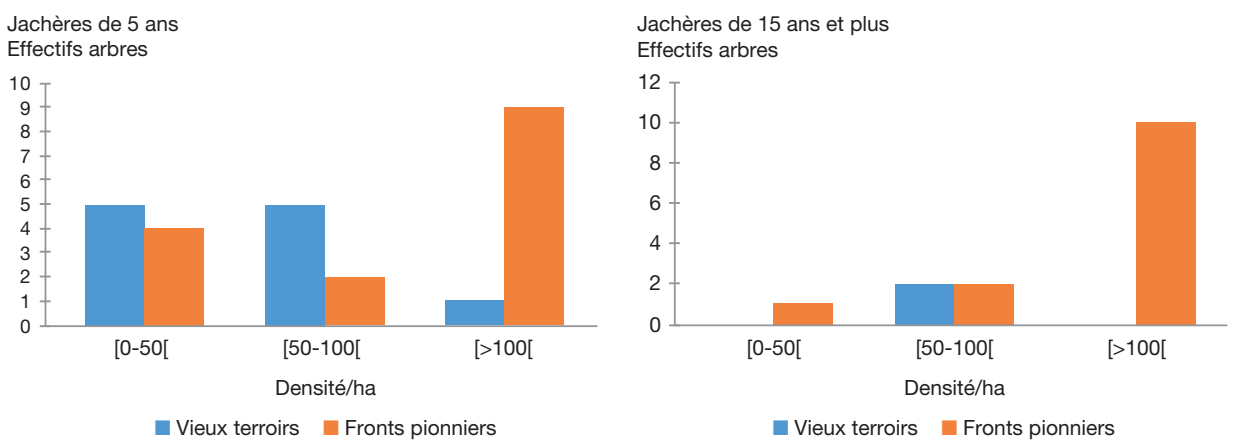

Figure 8.4. Densité de karités par hectare selon l'âge des jachères.

Source : enquête des auteurs, 2014. Anciens terroirs : villages de Selra et Tchiri. Fronts pionniers : villages de Guessou et Kirandi. Les karités pris en compte avaient au moins 1,30 m de hauteur.

Dans les jachères longues qui sont les plus propices à la régénération du karité, on compte plus de 50 pieds/ha de karité presque partout, souvent plus de 100 pieds/ha dans les villages de front pionnier. Dans les jachères courtes, la situation est surtout problématique dans les anciens terroirs où la densité moyenne de karités n'est que d'une trentaine de pieds à l'hectare. C'est insuffisant pour sélectionner les pieds productifs puisqu'une bonne part des karités s'avère être stérile, et une autre est peu productive.

Cependant, la diminution de la durée des jachères n'est pas la seule raison de la dégradation des parcs. En effet, l'absence de jeunes karités de moins de 15 ans dans les champs cultivés révèle aussi leur élimination systématique lors des mises en cultures. Dans cette région de Djougou, il n'y a pourtant pas de culture de cotonnier souvent associée à l'abattage des arbres, ni de mécanisation des travaux agricoles (avec la culture attelée par exemple) qui est aussi une raison de la destruction des plantules.

Cette absence de régénération du parc crée une situation probablement inédite : les parcs à karités sont devenus désormais un paysage hérité. Cet héritage est de plus en plus délabré, tandis que le vieillissement, les maladies et les parasites, ainsi que l'abattage pour du bois d'œuvre éclaircissent les peuplements.

\section{La typologie des champs, reflet de la dynamique des parcs}

Si la gestion du parc agroforestier se faisait autrefois sous la responsabilité de chefs de lignage et mettait en jeu des domaines souvent très vastes (Serpantié, 1996a), les décisions sont désormais prises par des chefs de famille disposant de tenures 
foncières plus petites. L'évolution du parc a suivi des trajectoires diverses qui peuvent être identifiées par les caractéristiques des champs. Sur les sites étudiés à Djougou, on peut distinguer quatre grands types en fonction de la densité des arbres, de la composition floristique, de la taille et de l'usage des champs :

- de grands champs à karités (0,6 ha et une moyenne de 30 arbres/ha, dont 22 karités). Ce sont les champs typiques d'un parc arboré à karités. Les karités dominent, accompagnés d'autres espèces comme le néré, quelques Khaya senegalensis et d'autres arbres utiles de forêt sèche;

- de grands champs à dominante de néré de 0,5 ha. La densité arborée est plus faible (21 arbres/ha), essentiellement par déficit de karités (5 karités/ha);

- de petits champs à recrû forestier (0,3 ha et 60 arbres/ha, dont 9 karités/ha). La plupart des arbres sont de petite taille (Hymenocardia acida, Prosopis africana et Parinari curatellifolia). Ces arbres sont utiles pour les fruits ou la pharmacopée, mais de faible valeur. On les laisse pousser, car ils ne gênent pas les cultures;

- des petits champs à anacardier (Anacardium occidentale) de 0,3 ha avec 74 arbres/ha dont 49 anacardiers/ha et 14 karités/ha. Limportante densité des anacardiers, presque toujours jeunes, semble montrer une transition rapide vers des vergers à anacardiers.

Les champs à karités datent de l'ancien cycle de jachère/culture, bien que ce cycle ait été interrompu il y a une vingtaine d'années par élimination des jeunes karités. Ces champs restent majoritaires puisqu'ils représentent près de la moitié de l'échantillon et sont représentatifs des fronts pionniers. Il suffirait donc de protéger une génération de jeunes karités issus des jachères pour régénérer le parc. Les champs à dominante de nérés semblent être un stade dégradé du type précédent. En effet, les karités disparaissent par abattage ou à cause des maladies. Il ne reste alors que les nérés qui, comme les karités, ne sont pas plantés, mais préservés lors de la défriche.

Les petits champs sont les plus nombreux dans les anciens terroirs. Cela montre une tendance au fractionnement des parcelles sous la pression des densités de populations croissantes. Les champs couverts d'un recrû forestier sont caractéristiques des jachères courtes.

Un peu partout, l'anacardier progresse pour diverses raisons. D'une part, étant un arbre planté (à la différence du karité ou du néré), il témoigne d'une revendication foncière et affirme les droits sur la terre. C'est la raison principale de sa progression sur les fronts pionniers où les droits fonciers de certains groupes sociaux sont quelquefois incertains. D'autre part, l'arbre entre rapidement en production (à 5 ans environ) et les prix des noix de cajou (anacardes) sont actuellement intéressants. Ceci est d'autant plus avantageux pour les hommes que le produit leur revient directement, sans passer par le biais des femmes, contrairement aux fruits du karité ou du néré.

\section{W Exploitation des karités : un miroir des inégalités intrafamiliales et sociospatiales}

Au Bénin comme dans l'ensemble de la zone d'extension du parc à karités, la gestion des ressources (terres, arbres, fruits, etc.) conserve le caractère genré décrit depuis la période coloniale. Le karité en est un archétype particulièrement significatif (Carney et Elias, 2006). Dans ces sociétés patriarcales, les hommes contrôlent la terre et les 
arbres qu'ils protègent ou coupent. Les femmes ont des droits d'usage sur des terres prêtées par leur mari ou leur père. Sur les arbres des champs ou des jachères du domaine familial, leurs droits portent sur la collecte des fruits, des écorces ou des feuilles, notamment du karité, du néré et du baobab. Les femmes les transforment éventuellement et les utilisent ensuite pour la consommation familiale ou les vendent (bruts ou transformés) pour couvrir les charges financières qui leur incombent, dans une économie familiale où les budgets sont en grande partie séparés.

\section{Une organisation marquée par des rôles genrés}

Les enquêtes socio-économiques réalisées en 2010 dans le cadre du projet Éclis auprès de 381 femmes ont permis d'estimer la place du karité dans les activités des femmes. Ainsi, $87 \%$ des femmes de l'échantillon tirent un revenu d'une activité liée au karité, le plus souvent par la vente de noix sèches, mais aussi, pour quelques-unes, par la fabrication et la vente de beurre de karité. Des observations similaires ont été faites par Schreckenberg (2004) qui a analysé l'importance du karité dans les moyens d'existence des populations rurales de la commune de Bassila, au sud de Djougou.

En 2014, une enquête complémentaire sur la conduite des activités liées au karité a été réalisée auprès d'un sous-échantillon de 101 femmes $^{3}$ parmi les 381 enquêtées en 2010 et ayant une activité liée au karité : elles sont réparties entre anciens terroirs et fronts pionniers. Cette enquête montre qu'à Djougou, les droits et l'organisation du travail restent apparemment assez conventionnels : les femmes collectent les fruits du karité sur les champs de leur propre famille. En revanche, l'accès aux jachères est beaucoup plus ouvert. Mais la concurrence est forte, y compris avec des enfants. Le ramassage se fait le matin, parfois avant l'aube. Elles se consacrent ensuite aux travaux dans les champs familiaux, puis aux travaux domestiques. Laide familiale vient seulement de leurs filles, sauf chez les Peuls où un tiers des maris aident pour le transport. Celui-ci est exclusivement manuel pour les femmes (cuvettes de $30 \mathrm{~kg}$ environ sur la tête), alors que les lieux de collecte se trouvent souvent à plusieurs kilomètres du village. Les fruits sont ensuite dépulpés, bouillis, séchés et décortiqués afin d'obtenir des amandes qui se conservent. Les quantités de noix ramassées par les femmes varient en fonction des localités. Dans les villages des anciens terroirs, près de la moitié des femmes collectent moins de $100 \mathrm{~kg}$ de noix, alors que dans les fronts pionniers, à Tchiri (Dabougou), plus du tiers des femmes ramasse $200 \mathrm{~kg}$ ou plus. Les femmes pourront les vendre ou les transformer en beurre.

La transformation du karité en beurre requiert un savoir-faire, du temps et des ressources en eau et en bois. En l'absence de mécanisation, notamment d'un moulin, cette opération est longue et pénible. Les usages alimentaires du karité se sont beaucoup réduits. En dehors des Peuls, la plupart des femmes de Djougou n'utilisent plus le beurre de karité pour la cuisine, le remplaçant par de l'huile de palme. En revanche, malgré une diminution, il reste présent dans les soins corporels (hydratation de la peau et des cheveux), comme cicatrisant et anti-inflammatoire dans la pharmacopée. Il est toujours utilisé dans de nombreux rituels, en particulier lors de cérémonies funéraires. Le beurre reste donc très associé à des activités de soins corporels ou care.

3. Bien que la polygamie soit possible et existe, une majorité de ménages est monogame, surtout chez les plus pauvres. Il n'a pas été possible de distinguer des différences entre épouses selon leur rang dans la pratique d'activités autour du karité. 


\section{Revenus tirés du karité et diversification des activités}

La commercialisation du karité remonte à plusieurs décennies, suivant la monétarisation des sociétés rurales d'Afrique de l'Ouest. Les femmes restent le premier maillon de la filière, surtout en tant que ramasseuses ${ }^{4}$, voire transformatrices, même si certaines se sont spécialisées aussi dans la collecte et la revente.

Les enquêtes réalisées en $2010^{5}$ à Djougou ont permis de replacer le karité dans l'ensemble des activités des femmes. En plus des activités agricoles dans les champs familiaux et des activités domestiques, les femmes mènent en moyenne cinq autres activités. Un quart d'entre elles en font plus de six, telles que la transformation de produits, la cueillette, le petit commerce, le salariat agricole.

Le revenu provenant de la vente des amandes de karité ou du beurre s'insère dans des proportions très variables dans les autres ressources monétaires des femmes, selon la localité et selon leur situation socio-économique. Cette part du karité ne dépasse pas $20 \%$ pour les deux tiers des femmes dans les terroirs anciens, celles-ci trouvent d'autres sources de revenus. Dans les fronts pionniers, les revenus monétaires tirés du karité tiennent une part plus importante pour les femmes rurales des villages enclavés parce que la densité de karités est plus importante, notamment dans les jachères, mais aussi et surtout parce que les opportunités de diversification, et donc de revenus, sont nettement plus faibles. Ce sont les femmes les plus pauvres qui dépendent le plus du karité pour leurs revenus monétaires.

Les revenus annuels générés par le karité sont, quant à eux, variables, mais faibles dans la majorité des cas. Pour $87 \%$ des femmes, la vente des noix sèches a rapporté moins de 25000 CFA (38€) en 2010, peu de femmes (3\%) ont gagné plus de 40000 CFA $(61 €)$.

La fabrication de beurre de karité est destinée à la consommation familiale. Parfois, une partie est vendue; certaines femmes achètent des noix pour fabriquer du beurre. Les vendeuses de beurre proviennent essentiellement des villages de fronts pionniers : Guessou et surtout Kirandi. À Kirandi, une femme enquêtée sur deux a vendu du beurre en 2013 et pour la moitié d'entre elles, pour plus de 40000 CFA.

Si on met en regard ces revenus avec ceux générés par les autres activités de la famille (production agricole, élevage et activités artisanales), on voit que la place économique du karité est hétérogène, souvent modeste. Ainsi, si pour la plupart des femmes pauvres des zones enclavées, les revenus du karité sont nécessaires faute d'autres opportunités, cela reste très peu rémunérateur compte tenu du temps consacré à la collecte, à la préparation des amandes et à la transformation; travaux dont la pénibilité est importante. Pour tirer un revenu considéré comme intéressant par rapport à d'autres opportunités, les femmes doivent avoir un peu de capital leur permettant d'acheter des noix ou des amandes pour les stocker et

4. Le terme de ramasseuse est couramment utilisé dans la description des acteurs de la filière; il comporte une certaine ambiguité, dans la mesure où, avant de vendre les noix, il y a un long travail de préparation des amandes demandant un certain savoir-faire.

5. La complexité des familles a été intégrée dans les enquêtes, notamment par la distribution des questionnaires auprès des femmes (mariées, séparées, veuves ou divorcées) membres des familles enquêtées, afin de prendre au mieux en compte la diversité des activités des femmes. 
les revendre lorsque les prix sont élevés ou disposer d'un accès à un moulin, et de débouchés rémunérateurs pour le beurre de qualité supérieure. Ce constat rejoint celui de Pouliot (2012) qui conclut, suite à une enquête réalisée sur la contribution du karité au revenu des femmes au Burkina Faso, que ceux-ci sont d'un apport certes utile, voire indispensable pour certaines femmes en période de soudure, mais sont insuffisants pour lutter contre la pauvreté, contrairement à ce que laisse entendre l'appellation couramment utilisée de «l’or des femmes» pour désigner le karité.

\section{Ressources et contexte familial}

Dans la région de Djougou, les transformations socio-économiques rapides de la société rurale depuis une trentaine d'années se sont accompagnées d'une aggravation des inégalités entre hommes et femmes (Droy et al., 2014). Avec une partie de leur temps consacré à leurs propres activités, les femmes gèrent des ressources indépendamment des hommes. Cela reflète la pluralité des unités de décision et la reconnaissance d'individus aux sphères d'activités plus ou moins autonomes à l'intérieur du ménage. Mais en replaçant les activités des femmes au sein de leur famille, on constate qu'elles sont en grande partie tributaires du degré de liberté dont elles disposent dans la famille, ainsi que des opportunités et souvent de l'aide qu'elles peuvent y trouver.

D'après les entretiens réalisés en 2014 auprès des femmes de Djougou, l'argent obtenu de la vente du karité permet d'assurer certaines dépenses de consommation courantes : achat de condiments, de vêtements et des produits de soin pour ellesmêmes et leurs enfants, voire couvrir les dépenses de santé et de scolarité des enfants — si l'homme ne les assure pas. Parfois, l'argent du karité sert directement à acheter de la nourriture, car il est disponible à une période de l'année où les familles rurales ont peu d'autres sources de revenu. Pour une minorité de femmes, ces revenus sont investis dans d'autres activités économiques.

Bien que de nombreuses femmes rappellent que «le karité, c'est notre source de richesse», et que «si la destruction des karités ne cesse pas, les femmes n'auront plus de revenus», elles se sentent assez démunies devant l'évolution de la situation. En effet, en dehors d'un consensus familial de plus en plus rare, elles n'ont pas de droit, ni même d'avis décisif sur le devenir des arbres, leur protection ou leur régénération. Or, les hommes, gestionnaires de la ressource, ont actuellement d'autres intérêts, comme la plantation de vergers d'anacardiers ou la coupe de karités pour le bois. Lavenir du parc à karités est aussi fortement dépendant de l'évolution des relations entre hommes et femmes. L'un et l'autre sont soumis à des mutations culturelles rapides.

\section{" Une filière mondialisée reposant sur le travail des femmes}

Le faible prix d'achat d'amandes de karité aux productrices reflète l'asymétrie de la chaîne de valeur des noix ou du beurre de karité au profit des maillons les plus élevés : les grands commerçants et les exportateurs.

Les chiffres de la production béninoise sont assez imprécis, mais celle-ci semble s'accroître. La FAO considère le Bénin comme un petit producteur avec une 
moyenne de $15000 \mathrm{t}$ noix/an, loin derrière les quatre grands pays producteurs d'Afrique de l'Ouest (Mali, Nigeria, Ghana et Burkina Faso), dont la production oscille autour des 100000 t noix/an. Pourtant, d'autres sources donnent des niveaux de production très supérieurs. Lassociation Karité Bénin (créé en 2013) estime que la production varie entre 40000 et 80000 t noix/an selon les années. La situation est donc assez confuse, mais semble évoluer assez rapidement sous l'effet d'une demande mondiale soutenue.

\section{Les opérateurs de la trituration et de l'exportation}

Depuis quelques années, l'essentiel de la production d'amandes se partage en deux sphères d'échange. La première est locale et produite pour des usages alimentaires, rituels ou cosmétiques. La seconde travaille pour l'exportation : les amandes sont destinées, pour l'essentiel, à l'industrie du chocolat, la stéarine du beurre de karité entrant comme substitut du beurre de cacao.

Mais récemment, le développement d'unités industrielles permet aussi de produire du beurre de karité de qualité alimentaire pour le marché local et international. La part respective de ces unités est mal connue, elle est aussi en rapide évolution.

La filière du karité est pourtant considérée comme risquée pour les différents opérateurs, en premier lieu les exportateurs et les industriels. Á l'amont de la filière, la collecte varie beaucoup du fait de l'irrégularité annuelle de la production des arbres et des volumes commercialisés. À l'aval, la filière est soumise de façon indirecte au marché mondial du cacao, car les prix de l'amande de karité s'alignent sur les cours du beurre de cacao, puis finalement sur ceux du cacao lui-même ${ }^{6}$. Lorsque les cours s'affaissent, les filières nationales du karité peuvent devenir non rentables en raison des coûts d'intermédiation élevés. Dans ces conditions, les achats ralentissent et éventuellement s'arrêtent. Les stocks s'accumulent alors.

Cette situation d'incertitude incite les acteurs de la filière à limiter leur spécialisation dans le karité. La diversification associe souvent la noix de cajou, quelquefois l'huile de palme ou de graines de coton. Chez certains commerçants indiens devenus exportateurs, les ventes d'amandes de karité et d'anacardes équilibrent les importations de tissus. Dans ces conditions, les transactions sur les amandes de karité ne représentent qu'une part, souvent minoritaire, dans les activités des entreprises ou des individus. L'essentiel du marché à l'export se partage entre deux entreprises, Knar-Bénin et Fludor, toutes deux filiales de firmes agroalimentaires internationales spécialisées dans les oléagineux.

\section{Les acheteurs : collecteurs et grossistes}

La chaîne d'opérateurs qui relie les exportateurs aux ramasseuses ou aux transformatrices a été bien décrite au Ghana (Wardell, 2013) et au Burkina Faso (Rousseau, 2016), plus rarement au Bénin (Gnanglè et al., 2012; Pourret, 2015).

6. Voir le site de Nkalo pour des informations et des synthèses sur plusieurs produits, dont le karité : http://nkalo.com/blog/karite/. Un suivi mensuel des prix à différentes étapes de la filière est réalisé dans plusieurs pays. 


\section{Diversité des collecteurs}

On observe une grande variété d'acteurs dans la collecte d'amandes de karité :

- des collectrices occasionnelles dans les villages, disposant d'un peu de trésorerie pour stocker et attendre une montée des prix;

- des petits commerçants du village plus ou moins liés à un grossiste, faisant éventuellement des prêts des denrées alimentaires aux ramasseuses;

- des collecteurs mandatés par une grande société.

Les coûts de transaction sont élevés en raison de l'absence d'organisation villageoise, ce qui renchérit le prix du transport. Comme souvent, de nombreux intermédiaires, plus ou moins scrupuleux (sur la pesée, sur le paiement, sur l'information), sont tentés de spéculer sur l'augmentation du prix des amandes au cours de la saison. Les ramasseuses ne bénéficient pas réellement de ces évolutions de prix, faute d'information, de manque de capacité de négociation et de possibilité de stockage.

La filière du karité est tributaire du respect d'un itinéraire technique préservant les qualités des amandes comme le tri à la récolte, les conditions de séchage après le dépulpage, l'ébouillantage pour éviter la germination dans les quatre jours suivant la cueillette. Or, beaucoup d'observations font état d'une proportion d'amandes avariées dépassant $50 \%$ lors des livraisons aux grossistes. Une grande partie des difficultés provient des ramasseuses elles-mêmes, car cet itinéraire est rarement respecté faute de formation et de temps. En effet, le ramassage a lieu en partie durant la saison des pluies; ce qui rend le séchage difficile. Cette période est aussi celle des travaux agricoles dans les champs. D'autres difficultés proviennent de la filière, avec les intermédiaires qui, en spéculant sur les prix, stockent les amandes dans des conditions inadéquates.

\section{Les grossistes-acheteurs}

Ils sont appelés «acheteurs» au Bénin, car ils sont enregistrés auprès du registre du commerce et des sociétés. On compte environ 200 acheteurs qui collectent, stockent et transportent les amandes de karité, en plus de divers produits agricoles. Ces acheteurs sont regroupés au sein du groupement des acheteurs de produits agricoles (Gapa). Ils sont protégés par la réglementation béninoise interdisant aux négociants-exportateurs de traiter directement avec les villageois, les obligeant à passer par ces acheteurs. Certains exportateurs, comme Knar-Bénin, ont des contrats avec ces acheteurs.

Faute de données fiables, la répartition de la valeur ajoutée sur l'ensemble de la filière est assez mal connue; les relevés de Pourret (2015) montrent tout de même que le prix à la tonne double entre le prix d'achat des collecteurs aux productrices et le prix de vente aux acheteurs. Ces commerçants ont souvent aussi d'autres fonctions : l'approvisionnement du monde rural en biens de consommation et le crédit plus ou moins usuraire, selon un mode de fonctionnement caractéristique d'une économie de traite auquel le milieu rural reste toujours inféodé (Badouin, 1967).

\section{Des initiatives pour mieux structurer la filière : au profit de qui?}

Les initiatives pour la structuration de la filière sont récentes, avec une impulsion venant essentiellement des acteurs de l'aide internationale poussant aux réformes institutionnelles de la filière du karité (Wardell, 2013). 
Au niveau international, la Global shea alliance (GSA), créée en 2011, est une association industrielle à but non lucratif basée à Accra, au Ghana. Elle compte actuellement 400 membres incluant des firmes internationales agroalimentaires et cosmétiques, des petites entreprises, des ONG et des groupements de femmes. Son but affiché est de promouvoir le karité dans le monde en rendant la filière compétitive et durable et, de façon pratique, en promouvant une politique de qualité. $\mathrm{Au}$ niveau national, sur le même modèle, l'association Karité-Bénin réunit sur une plateforme commune les acteurs engagés dans la filière, souvent aux intérêts divergents : groupements de ramasseuses et de transformatrices des noix de karité, groupements d'acheteurs professionnels, industriels ou petites entreprises.

Au Bénin, une caractéristique fondamentale de la filière du karité est l'atomisation et l'absence de structuration en amont, au niveau de la production (les ramasseuses et les transformatrices). Sur le modèle des autres filières agricoles, la création de groupements paraît être une solution pour supprimer certains maillons intermédiaires. En 2015, il y aurait environ 200 groupements de femmes travaillant essentiellement dans le ramassage des noix de karité ou, plus rarement, dans la transformation en beurre. Pour la plupart issus d'un projet de développement, il est difficile de savoir si ces groupements sont encore tous actifs ou même organisés. Les freins sont nombreux : illettrisme des femmes, faiblesse de la gestion coopérative, autonomie limitée par rapport aux acheteurs et insuffisance des équipements. Comme cela a été observé au Burkina Faso, l'absence de transparence et de participation des membres aux processus de décision incite à relativiser le rôle de ces organisations en tant que moyen d'accroître le pouvoir d'action des femmes ou comme outil de lutte contre la pauvreté (Saussey et al., 2008).

De fait, le principal objectif des opérateurs semble être la formation des femmes aux bonnes pratiques de ramassage, de conservation et de décorticage : c'est-à-dire, l'essentiel de l'itinéraire technique qui repose sur les femmes, sans s'attaquer réellement à la répartition de la valeur au sein de la filière. Les mécanismes de l'extorsion du surtravail des femmes, bien analysés dans ces contextes (Michel, 1985), semblent toujours à l'œuvre.

Conscient de ce problème de faible rémunération des collectrices, le projet ProAgri (Promotion de l'agriculture au Bénin), appuyé par la GIZ, a mis en place en 2015 des groupements de ramasseuses qui travaillent directement en relation avec l'industriel Fludor. Cette organisation limite les intermédiaires et permet de mieux rémunérer les productrices, tout en assurant à l'acheteur une meilleure maîtrise de la qualité.

\section{- Conclusion}

Au Bénin, ce n'est que récemment que le karité a été considéré comme une ressource méritant une politique de développement. Jusque dans les années 2000, bien qu'étant reconnu comme troisième poste d'exportation du pays, il n'avait pas suscité d'intérêt particulier. Certes, le parc à karités continue à s'étendre du fait des défrichements encore actifs sur la forêt, mais pour l'essentiel, dans les zones mises en culture depuis plusieurs décennies, le parc n'est plus régénéré. Deux 
causes fondamentales sont à envisager. D’une part, la croissance de la population rurale et son corollaire la réduction des jachères longues qui sont nécessaires au renouvellement du parc. D'autre part, le désintérêt des paysans qui trouvent dans l'anacardier un arbre plus rentable, même si ses mérites écologiques sont bien moins évidents ${ }^{7}$. Les initiatives de régénération assistée des karités, pourtant utiles, n'apportent donc que des réponses partielles à ces deux problèmes. D'autant plus que dans de nombreux lieux, dont Djougou, les jeunes plants de karité sont systématiquement éliminés des champs. Une gestion renouvelée des parcs arborés semble nécessaire, comme la création de conventions locales de gestion des parcs arborés permettant d'aborder d'une façon globale la question de la dégradation des parcs arborés à karités pour relever ces défis. Ainsi, les conventions locales de gestion des ressources naturelles sont apparues depuis les années 1990 dans les pays engagés dans un processus de décentralisation. Touré (2011, p. 2) écrit : «les conventions locales peuvent être définies comme des accords légitimes négociés entre plusieurs parties prenantes (stakeholders) dans une perspective de régulation des ressources naturelles en termes de contrôle, d'accès, d'appropriation, d'usage et d'exploitation».

Certes, l'argumentaire des opérateurs du développement sur le karité s'inscrit à la jointure de la rhétorique du développement et de celle du genre. Il stipule qu'en fournissant des noix et du beurre de karité pour le marché international, les femmes pauvres accroissent leur revenu; en le faisant dans des associations, elles s'affirment dans leur rôle communautaire pour leur propre bien-être et celui de leurs enfants. Et par conséquent, du fait de son intérêt économique, le parc à karités sera entretenu et protégé. Cependant, ce cercle vertueux est loin de se réaliser (Saussey et al., 2008). À Djougou, pour les femmes qui ramassent les noix de karité, la vente ne représente une part significative de revenu que pour les plus pauvres ou celles qui habitent les villages les plus enclavés, et n'ont pas d'alternative plus rentable. La relance du ramassage des noix ou de la fabrication de beurre, de surcroît en utilisant un itinéraire technique garantissant la livraison de produits de qualité, ne peut être envisagée qu'en les rémunérant décemment et donc en agissant sur la chaîne de valeur permettant de rendre le maintien du parc arboré de nouveau attractif pour les populations rurales.

\section{" Bibliographie}

Agbahungba G., Depommier D., 1989. Aspects du parc à karités-nérés (Vitellaria paradoxa Gaertn. F., Parkia biglobosa Jacq. Benth.) dans le sud du Borgou (Bénin). Revue bois et forêts des tropiques, $222:$ 41-54.

Andrieu J., Mering C., 2008. Cartographie par télédétection des changements de la couverture végétale sur la bande littorale ouest-africaine : exemple des rivières du sud du delta du Saloum au Rio Geba. Télédétection (Éditions scientifiques), 8(2) : 93-118.

Badouin R., 1967. Où en est la réforme de l'économie de traite en Afrique noire? Tiers-Monde, $8(32): 1209-1216$.

7. Les entretiens réalisés dans le cadre du projet Safse montrent que l'intérêt pour le parc à karités diffère selon les parties : les femmes, les personnes âgées et les animistes étant plutôt attachés au parc et déplorant sa dégradation. 
Bidou J.-E., Droy I., Houesse R., Mering C., 2018. Dynamiques démographiques, vulnérabilité et évolution du couvert végétal au Nord-Bénin : des interactions complexes. Espaces, populations, sociétés, 3-4.

Boffa J.-M., 2000. Les parcs agroforestiers en Afrique de l'Ouest : clés de la conservation et d'une gestion durable. Unisylva, $51: 11-17$.

Carney J., Elias M., 2006. Revealing gendered landscapes: indigenous female knowledge of agroforestry of African shea. Canadian journal of african studies, 40(2): 235-267.

Droy I., Pascual C., Bidou J.E., 2014. Inégalités de genre et vulnérabilité alimentaire en milieu rural béninois : des interactions complexes. In : Guetat-Bernard H. et Saussey M. (eds). Genre et savoirs. Pratiques et innovations rurales au Sud. Paris, France : IRD éditions, 85-115.

Garrity D.P., Akinnifesi F.K., Ajayi O.C., Weldesemayat S.G., Mowo J.G., Kalinganire A., Larwanou M., Bayala J., 2010. Evergreen agriculture: a robust approach to sustainable food security in Africa. Food security, 2(3): 197-214.

Gnanglè C., Yabi J.A., Yegbemey N.R., Kakaï R.G., Sokpon N., 2012. Rentabilité économique des systèmes de production des parcs à karité dans le contexte de l'adaptation au changement climatique du Nord-Bénin. African crop science journal, 20(s2) : 589-602.

Kakaï R.G., Akpona J.D., Assogbadjo A.E., Gaoué O.G., Chakeredza S., Gnanglè P.C., Mensah G.A., Sinsin B., 2011. Ecological adaptation of the shea butter tree (Vitellaria paradoxa C.F. Gaertn.) along climatic gradient in Benin, West Africa. African journal of ecology, 49: 440-449.

Lovett P.N., Haq N., 2000. Evidence for anthropic selection of the Shea nut tree (Vitellaria paradoxa). Agroforestry systems, 48(3): 273-288.

Mahlstein K., MacDaniel C., 2016. Une bonne affaire : comment les modifications des règles d'étiquetage aux États-Unis pourraient bénéficier à l'Afrique. Passerelles, 17(9) : 1-5.

Michel A., 1985. La sortie du travail invisible : les femmes dans l'économie. Revue Tiers-Monde, 26(102) : 161-273.

Ouédraogo S.J., Devineau J.L., 1997. Rôle des jachères dans la reconstitution du parc à karité (Butyrospermum paradoxum Gaertn. F. Hepper) dans l'Ouest du Burkina Faso. In : Floret C. (ed.) La jachère, lieu de production. Actes du séminaire, Bobo-Dioulasso, Burkina Faso, 2-3 octobre 1996, Coraf-Union européenne. Bobo Dioulasso, Burkina Faso : CNRST-Orstom, 81-87.

Pélissier P., 1980. Larbre dans les paysages agraires de l'Afrique noire. Cahiers Orstom, (série Sciences humaines), 17(3-4) : 131-136.

Pouliot M., Treue B., 2012. Rural people's reliance on forests and the non-forest environment in West Africa: evidence from Ghana and Burkina Faso. World development, 43: 180-193.

Pouliot M., 2012. Contribution of "women's gold" to West African livelihoods: the case of shea (Vitellaria paradoxa) in Burkina Faso. Economic botany, 66(3): 237-248.

Pouliot M., Elias M., 2013. To process or not to process? Factors enabling and constraining shea butter production and income in Burkina Faso. Geoforum, 50: 211-220.

Pouret M., 2015. Appui à la commercialisation pour les chaines de valeurs de la filière karité au Bénin. Programme de promotion de l'agriculture au bénin. Cotonou, Bénin, GIZ, 34 p. + annexes. Rongead, 2014. Vitellaria paradoxa, http://rongead.org/IMG/pdf/karite_15.09.pdf, 21 p.

Rousseau K., Gautier D., Wardell A., 2015. Coping with the upheavals of globalization in the shea value chain: the maintenance and relevance of upstream shea nut supply chain organization in western Burkina Faso. World development, 66: 413-427.

Rousseau K., 2016. Political ecology du karité : relations de pouvoir et changements sociaux et environnementaux liés à la mondialisation du commerce des amandes de karité. Cas de l'Ouest du Burkina Faso. Environnement et Société. Thèse de l'Institut des sciences et industries du vivant et de l'environnement (AgroParisTech), 237 p.

Saussey M., Maïzi P.M., Muchnik J., 2008. Nouvelles formes de reconnaissance des femmes burkinabè dans la production collective de beurre de karité. Cahiers agricultures, 17(6) : 582-586.

Saussey M., 2011. Initiatives féminines et économie sociale et solidaire dans la production du beurre de karité au Burkina Faso. In : I. Guérin I., Hersent M., Fraisse L., (eds). Femmes, économie et développement. De la résistance à la justice sociale. Eres, 107-128. 
Schreckenberg K., 2004. The contribution of shea butter (Vitellaria paradoxa C.F. Gaertner.) In: Sunderland T., Ndaye O., (eds). Forest products, livelihoods and conservation. Case studies of nontimber forest products systems. Vol. 2. Africa. Djakarta: Cifor, 91-113.

Serpantié G., 1996a. La production de karité (Butyrospermum paradoxum Gaertn. F. Hepper) des parcs arborés de l'Ouest-Burkina Faso. Effets de différents modes de gestion. In : Floret C. (ed.) La jachère, lieu de production. Actes du séminaire, Bobo-Dioulasso, Burkina Faso, 2-3 octobre 1996. Bobo Dioulasso, Burkina Faso : CNRST-Orstom, 73-80.

Serpantié G., 1996b. Rôles des jachères dans la production arborée non ligneuse en savane soudanienne. Cas du karité dans l'ouest du Burkina Faso. In : Floret C. (ed.) La jachère, lieu de production. Actes du séminaire, Bobo-Dioulasso, Burkina Faso, 2-3 octobre 1996. Bobo Dioulasso, Burkina Faso: CNRST-Orstom, 55-57.

Serpantié G., Bayala J., Helmfrid S., Lamien N., 1996. Pratiques et enjeux de la culture du karité (Butyrospermum paradoxum Gaertn. F. Hepper) dans l'Ouest du Burkina Faso. In : Floret C. (ed.) La jachère, lieu de production. Actes du séminaire, Bobo-Dioulasso, Burkina Faso, 2-3 octobre 1996. Bobo Dioulasso, Burkina Faso: CNRST-Orstom, 59-72.

World bank, 2015. The Republic of Benin: diagnostic trade integration study (DTIS) update: from rents to competitiveness. Report $n^{\circ}$ 97242-BJ. Final report, Mai 2015. Trade and competitiveness global practice. Africa region. Document of the World Bank.

Touré E.H., 2011. Les conventions locales pour la gestion des ressources naturelles au Sénégal : entre autonomisation et problème d'appropriation. VertigO, 11(1).

Walla K., Sinsin B., Kudzo A.G., Kouami K., Akpagana K., 2005. Typologie des systèmes agroforestiers dans la préfecture de Doufelgou (Togo). Sécheresse 16(3) : 209-216.

Wardell A., 2013. Globalisations in a nutshell: historical perspectives on the changing governance of the shea commodity chain in northern Ghana. International journal of the commons, 7(2): 367-405. 



\title{
Chapitre 9
}

\section{Consommation en eau d'une espèce agroforestière en zone soudanienne}

\author{
Awessou Kohomlan G.-B., Peugeot C., Agbossou E. et Seghieri J.
}

Résumé. En zone soudanienne, les cultures sont pratiquées depuis des siècles par les populations rurales sous forme d'agroforesterie. Cette pratique est dominée par les parcs à karités (Vitellaria paradoxa), en alternance avec les jachères dans lesquelles les arbres se régénèrent. Pour évaluer les possibilités de densification en karité de ces parcs, nous avons quantifié la transpiration à l'échelle de l'arbre et à celle de la population de karité au sein d'une jachère de dix ans située au nord du Bénin. La densité de flux de sève a été mesurée par la méthode de dissipation thermique transitoire. La transpiration à l'échelle de l'arbre a été déduite de la densité de flux de sève puis extrapolée à celle de la population. Les résultats des trois années d'étude (2011-2013) montrent que la transpiration d'un arbre augmente de 4 à 27 l/jour en fonction de son diamètre $(8$ à $38 \mathrm{~cm})$. La transpiration varie peu entre la saison des pluies et la saison sèche, suggérant que l'eau n'est pas le facteur limitant. Mais elle diminue brutalement au moment du renouvellement des feuilles (février-mars). À l'échelle de la population de karités, la transpiration reste très faible $(0,03 \mathrm{~mm} /$ jour $)$ et représente $0,42 \%$ à $1,32 \%$ de la demande atmosphérique estimée par l'évapotranspiration de référence, et $1,15 \%$ de la pluie annuelle. Ces résultats indiquent qu'une augmentation importante de la densité des arbres est possible. Cependant, la seule estimation des besoins en eau des karités reste insuffisante pour améliorer la gestion des parcs agroforestiers et intensifier leur production. Il faudra en effet faire des compromis entre la densification des arbres et l'impact de la lumière incidente filtrée par leur canopée sur la limitation de la production de la culture associée.

\begin{abstract}
For centuries, in the Sudanese belt, rural populations have been practicing agroforestry dominated by shea trees (Vitellaria paradoxa) parklands as the main farming system alternating with fallows where trees regenerate. To estimate the margin of the tree density increase in shea parklands in northern Benin, we quantified shea transpiration, as indicator of shea water needs, in a 10-year old fallow at the tree and plot scales. Sap flow density (SFD) was measured by the transient thermal dissipation method from which transpiration of the tree was deduced then extrapolated at the shea cover scale. Over the
\end{abstract}


three-year of the study period (2011-2013), daily tree transpiration increased from 4 to $27 \mathrm{l}$ /day according to tree diameter ( 8 to $38 \mathrm{~cm}$ respectively). Transpiration lowly varied between wet and dry seasons, suggesting that water is not the limiting factor, except a sharp drop during the period of leaves renewal (February-March). Transpiration of the cover was very low $(0.03 \mathrm{~mm} /$ day $)$, corresponding to 0.42 à $1.32 \%$ of the atmospheric demand estimated by reference évapotranspiration Eto, and $1.15 \%$ of the annual rainfall. These results indicate that an important shea tree density increase is possible. Quantification of shea tree water needs alone will not help to improve parkland management and increase shea production. Trade-offs between trees density and light decrease for crop by tree canopies must be taken into account.

\section{Introduction}

Depuis plusieurs décennies, sous une pression démographique croissante, la zone soudanienne est soumise à une augmentation des superficies cultivées au détriment des jachères et des forêts. Entre 1990 et 2000, elle a connu une diminution de 1,5 million d'hectares de forêt et de 6 millions d'hectares de savane au profit de couverts plus ouverts, principalement des champs et des jachères (Bodart et al., 2013). Les parcs agroforestiers dans lesquels coexistent le karité (Vitellaria paradoxa) et le néré (Parkia biglobosa) sont très répandus. Ils succèdent aux écosystèmes forestiers (Bayala et al., 2008). Ces parcs sont le résultat de pratiques agricoles millénaires qui associent aux arbres, sur les mêmes parcelles, une ou plusieurs cultures annuelles, principalement le maïs, le sorgho et l'igname. Les noix de karité sont riches en matière grasse et servent à la fabrication du beurre. Elles constituent une source vitale de revenus pour les productrices et alimentent des filières d'exportation plus ou moins organisées et structurées dans plusieurs pays d'Afrique de l'Ouest dont les principaux sont le Ghana, le Bénin, le Mali et le Burkina Faso. En zone soudanienne, la superficie des parcs à karité est passée de 85000 ha en 1961 à plus de 600000 ha en 2016 (Seghieri, 2019). Parallèlement, la densité des karités a diminué drastiquement dans les parcs : de 230 arbres/ha en 1940 (Chevalier, 1946) à 11 arbres/ha en 2003 (Nikiéma et al., 2003). Cette baisse est attribuée à la réduction de la durée des jachères, qui se traduit par la baisse importante du taux de régénération des karités (Nikiéma, 2005).

Une des questions à résoudre, engendrée par l'introduction de la régénération assistée, toujours d'actualité dans plusieurs pays, est celle d'un compromis à faire entre l'augmentation de la densité des arbres pour intensifier la production de fruits et de noix de karité et le risque de compétition pour la lumière, l'eau et les nutriments entre les arbres et les cultures associées (Ong et al., 2014). Ainsi, les agriculteurs privilégient spontanément des densités d'arbres assez faibles, de sorte que cette compétition est largement évitée. Cependant, les connaissances scientifiques du fonctionnement des parcs à karités (impacts des arbres, interactions entre les arbres et les cultures, etc.), les enjeux environnementaux, économiques et sociétaux de l'intensification de leurs productions restent très limitées. En particulier, la connaissance des conditions d'augmentation durable de la production du karité est primordiale pour maintenir les autres «services» fournis par le parc. Dans ce contexte, notre étude a pour objectif de mieux cerner les possibilités de densification des arbres compte tenu de leur consommation en eau et des disponibilités hydriques du milieu. 
Concrètement, la transpiration de plusieurs arbres de tailles différentes a été estimée et extrapolée à l'échelle du peuplement spécifique de karités dans une jachère de 10 ans. La transpiration indique la consommation en eau du karité à ces deux échelles. À partir de cette étude de cas, les possibilités de densification d'un parc cultivé à karités susceptible de succéder à la jachère sont évaluées.

Les premiers résultats ont été publiés en 2017 (Awessou et al., 2017). À notre connaissance, auparavant quatre études ont quantifié la consommation en eau du karité, à l'échelle de l'individu ou du peuplement. Compaoré (2006) a estimé la transpiration du karité, espèce dominante dans un couvert ligneux multi-spécifique au Ghana (Navrongo). Bayala et al. (2008) ont estimé la transpiration à l'échelle de l'arbre dans un parc agroforestier à karités au Burkina-Faso. Ils ont mis en évidence l'existence d'une redistribution hydraulique, c'est-à-dire la remontée verticale de l'eau des horizons profonds et plus humides du sol à travers le système racinaire des arbres et son relargage dans les horizons de surface plus secs au bénéfice des arbres et des cultures associées. Tia (2008) a estimé la transpiration du karité à l'échelle de l'arbre et de la populaion, dans une étude localisée au sud-ouest du Burkina-Faso. Il a ensuite évalué, par modélisation, la contribution du karité à l'évapotranspiration du peuplement ligneux multispécifique. Au cours d'une étude conduite au Burkina Faso (Gonsé), Bayala et al. (2009) ont comparé la variation saisonnière de la transpiration de jeunes plants de karité âgés de 8 ans provenant du Burkina Faso, du Mali et du Sénégal.

\section{Matériels et méthodes}

\section{Le site expérimental}

Leétude a été réalisée sur le bassin versant de la Donga, Bénin (fig. 9.1), à $450 \mathrm{~km}$ au nord-ouest de Cotonou (940' - 9॰54'N, 1³4' - 158' E; fig. 9.1a). Dans ce site, la saison des pluies s'étend d'avril à octobre, la pluviométrie moyenne était de $1200 \mathrm{~mm} / \mathrm{an}$ de 1950 à 2005. Comme à l'échelle régionale, la superficie des forêts claires est passée de $60 \%$ en 1973 à moins de $40 \%$ en 2000 dans ce bassin au profit des surfaces cultivées $(+12,5 \%)$ et des jachères $(+6 \%)$ (Leroux, 2012). Dans la zone d'étude, le karité domine largement le couvert ligneux dans les parcs agroforestiers et les jachères.

Le site expérimental est une jachère de 10 ans d'environ 1,8 ha composée d'herbacées annuelles et pérennes, d'arbustes et d'arbres de plusieurs espèces avec une dominance net en karités, évaluée grâce à un inventaire préalable (fig. 9.1b).

\section{Le dispositif et la méthode d'estimation de la transpiration}

La jachère étudiée est constituée de neuf espèces végétales selon notre inventaire en 2013. Seuls les karités (espèce dominante de la jachère) ont été instrumentés pour la mesure de la transpiration. Ainsi, le couvert spécifique fait référence au couvert du karité considéré dans ce cas comme un couvert «pur» de karités.

Lestimation de la transpiration du karité est réalisée à partir d'un échantillon de 5 arbres dont les dimensions sont représentatives de la gamme des dimensions 
mesurées lors de l'inventaire (tab. 9.1). Le flux de transpiration d'un arbre est estimé à partir de la densité de flux de sève exprimée en litres par unité de surface d'aubier (partie du tronc dotée de vaisseaux fonctionnels) et par jour $\left(1 / \mathrm{dm}^{2} / \mathrm{j}\right)$. Lintégration de la densité de flux de sève sur la surface d'aubier fournit la transpiration à l'échelle de l'arbre.

La densité de flux de sève est estimée par la méthode de dissipation thermique transitoire proposée par Do et Rocheteau (2002) et Isarangkool Na Ayutthaya et al. (2010). Elle est dérivée de la méthode à chauffage continu de Granier (1985) : la différence de température est mesurée en continu entre une sonde chauffée insérée dans l'aubier à hauteur de poitrine et une sonde inerte insérée à $5 \mathrm{~cm}$ plus bas. Les données sont stockées dans une centrale d'acquisition Campbell.

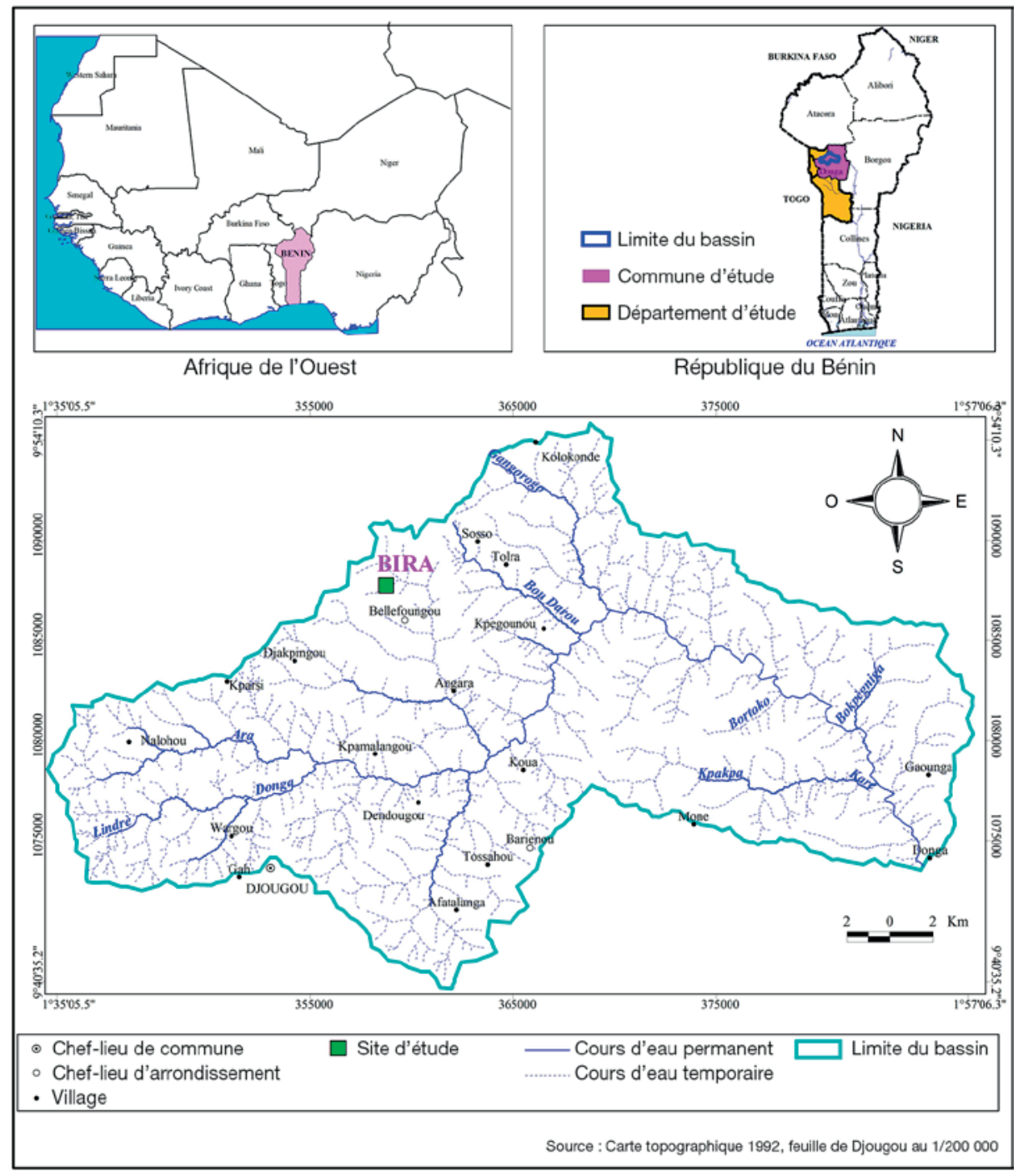

Figure 9.1a. Localisation du site d'étude : Bassin versant de la Donga. 


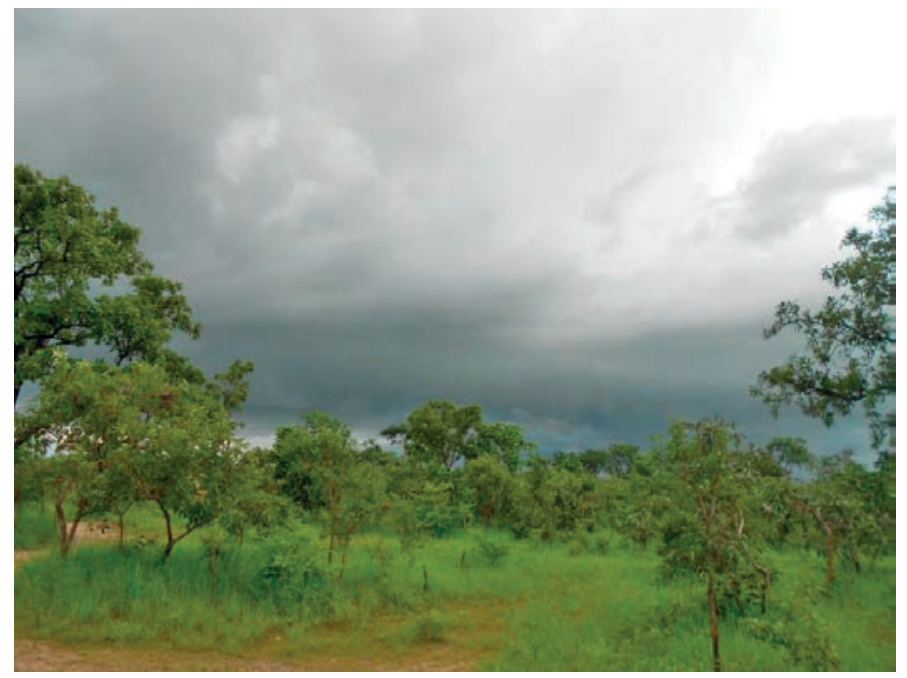

Figure 9.1b. Jeune jachère de Bira (ㄷ Marie Bellanger)

Le flux de sève ascendant dissipe la chaleur produite par la sonde chauffée et réduit la température dans son voisinage. Par conséquent, plus grande est la différence entre les deux sondes, plus faible est la densité de flux de sève. Dans la méthode transitoire, les phases de chauffage (10 minutes) alternent avec celles de refroidissement (20 minutes). Le chauffage discontinu permet de corriger le biais lié aux conditions extérieures qui peut conduire à des erreurs importantes dans l'estimation du flux de sève (Do et Rocheteau, 2002). Un étalonnage valable pour toutes les espèces permet de déduire la densité de flux de sève du différentiel de température (Do et Rocheteau, 2002).

La densité de flux de sève décroît de la périphérie vers le centre de l'aubier à cause de la diminution des propriétés conductrices de l'aubier du fait de la production de bois mort au centre du tronc (Jiménez et al. 2000; Nadezhdina et al., 2002). Ce profil radial de la densité de flux de sève est pris en compte pour éviter, là encore, des biais importants dans l'estimation de la transpiration (Nadezhdina et al., 2002). Lorsque toute la profondeur de l'aubier n'est pas explorée par les sondes, un facteur de correction est appliqué aux mesures (Awessou et al., 2017). La surface d'aubier d'un arbre est déduite des mesures de son épaisseur obtenue par des carottages dans le tronc à hauteur de poitrine et parallèlement au sol à l'aide d'une tarière de Pressler.

Plusieurs sondes ont été implantées à différentes profondeurs sur les deux plus gros arbres de notre échantillon (diamètre à hauteur de poitrine, $\mathrm{DHP}>30 \mathrm{~cm}$, tab. 9.1), de sorte à intégrer toute l'épaisseur d'aubier. Pour les trois autres arbres plus petits, seule la partie externe de l'aubier, où la densité de flux de sève est maximale, a été instrumentée. La transpiration des deux plus gros arbres est le produit de la densité de flux de sève externe, corrigée par la surface totale de l'aubier afin de prendre en compte le profil radial (Awessou et al., 2017). La transpiration des trois plus petits arbres est calculée directement par le produit de la densité de flux de sève externe et la surface totale d'aubier. 
Pour estimer la transpiration de la population ou le couvert de karité de la parcelle ${ }^{1}$, la transpiration estimée sur chacun des cinq arbres a été extrapolée à tous les individus inventoriés en faisant les hypothèses suivantes :

- les individus de même diamètre ont la même surface d'aubier (Pataki et Oren, 2003; Kumagai et al., 2007);

- les cinq arbres échantillonnés représentent suffisamment bien le peuplement de karité.

Linventaire a permis de dénombrer 17 arbres/ha. Leur surface totale d'aubier a été estimée à $0,11 \mathrm{~m}^{2} / \mathrm{ha}$. La densité de flux de sève moyenne, calculée sur les cinq arbres instrumentés, est extrapolée à l'échelle de la population de karité selon l'équation ci-dessous :

Tr couvert $=\mathrm{J}_{\mathrm{s}} \times \mathrm{A}_{\mathrm{s}} / \mathrm{A}_{\mathrm{g}}$

Tr couvert (mm/jour) est la transpiration de la population de karité sur la parcelle.

Js $\left(1 / \mathrm{m}^{2} /\right.$ jour $)$ est la densité de flux de sève (DFS) moyenne des cinq arbres instrumentés, calculée comme la somme de la transpiration des cinq arbres divisée par la somme de leurs surfaces d'aubier. Au préalable, il a été vérifié que la relation entre la transpiration à l'échelle de l'arbre et le diamètre de l'arbre est linéaire de $1^{\mathrm{er}}$ ordre.

As $\left(\mathrm{m}^{2}\right)$ est la surface de bois d'aubier de tous les karités de la parcelle.

$\mathrm{Ag}\left(\mathrm{m}^{2}\right)$ est la surface de la parcelle $\left(\mathrm{m}^{2}\right)$.

La demande évaporatoire de l'atmosphère, qui est le déclencheur du processus de transpiration, a été approchée par l'évapotranspiration de référence (Eto) calculée à l'aide de la formule de Penman-Monteith (Allen et al., 1998).

\section{" Résultats et discussion}

\section{La densité de flux de sève}

Quel que soit l'arbre considéré — et comme attendu —, la densité de flux de sève augmente rapidement dès le lever du soleil. Sa valeur maximale est atteinte entre 12 et 15 h (UTC, fig. 9.2). Puis, elle diminue et devient nulle entre 18 h et 6 h (fig. 9.2). La densité de flux de sève d'un arbre est toujours un peu plus élevée en saison des pluies qu'en saison sèche (tab. 9.1 et fig. 9.2).

Sur la période d'étude (2011-2013), la densité de flux de sève varie d'un arbre à l'autre de 5 à 11 1/. $\mathrm{dm}^{2}$ jour pour des diamètres allant de 8,3 à 38,2 cm (tab. 9.1). La densité de flux de sève de l'arbre «V.p3» est inférieure à celle de l'arbre «V. p1 », malgré un diamètre à hauteur de poitrine (DHP) et une surface d'aubier plus grands. Une différence comparable est observée entre les arbres «V.p2» et «V.p8».

Cette variation interindividuelle de la densité de flux de sève est sans doute à relier à la variation de la conductivité hydraulique. Celle-ci est fonction, d'une part, du diamètre et de la densité des vaisseaux du xylème (Tateishi et al., 2008) et, d'autre

1. Le couvert de karité sous-entend la population de karités dans la jachère. Le karité étant une espèce naturelle, les individus sont dispersés dans la jachère. Son couvert représente donc l'ensemble des karités dans la jachère. Sur le site d'étude, nous avons dénombré 17 arbres/ha. Les valeurs de transpiration qui sont données dans la suite du document ne concernent que la transpiration de la population de karités et non la transpiration du peuplement de la jachère. 


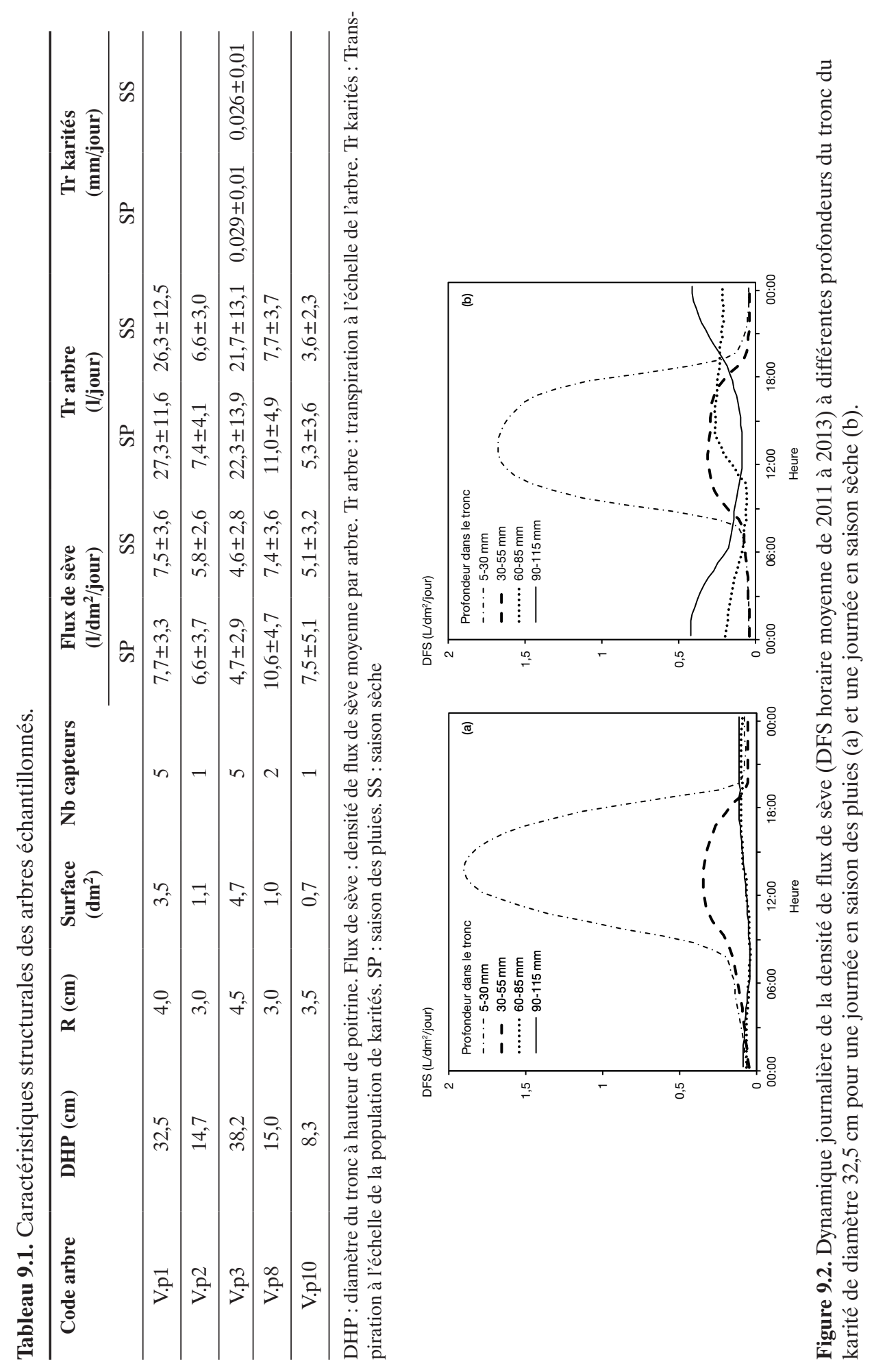


part, du rapport entre la surface foliaire et celle de l'aubier (West et al., 1999; Issoufou et al., 2015). La résistance hydraulique existant entre le sol et la canopée peut également avoir une influence car elle varie en fonction de la hauteur de l'arbre et de la perméabilité de l'aubier (Venkatraman et Ashwath, 2016). Enfin, l'état sanitaire et la vigueur des arbres, en lien avec leur âge plus ou moins avancé, peuvent influencer l'état des vaisseaux, et donc la transpiration.

\section{La transpiration}

À l'échelle de l'arbre et à celle du couvert, la transpiration chute brutalement en février-mars, période correspondant au renouvellement des feuilles (fig. 9.3b). Nos observations phénologiques montrent que cette défeuillaison concerne environ $50 \%$ de la canopée.

Une tendance à la baisse de la transpiration de la population de karité entre 2011 et 2013 s'observe sur la figure 9.3c et sur les valeurs normées par la demande atmosphérique (Eto) (fig. 9.3d). Cette tendance est attribuée à un artefact généré par la mise en place par l'arbre de tissus cicatriciels autour des capteurs en réaction à l'insertion des sondes. Cette réaction bien connue (Wiedemann et al., 2016) modifie les propriétés thermiques du bois et semble particulièrement forte sur les karités étudiés ici. Elle induit une sous-estimation des valeurs absolues du flux de sève dans les 2 à 3 ans qui suivent l'installation des sondes. En l'absence de données physiques de ce phénomène, nous n'étions pas en mesure de corriger cette dérive.

\section{Intensité de la transpiration moyenne journalière d'un arbre}

Au cours des trois années d'étude et sur les cinq arbres instrumentés, l'intensité de la transpiration moyenne journalière d'un arbre varie de 4 à 27 1/jour et augmente de façon linéaire avec son diamètre $\left(\mathrm{R}^{2}=0,87\right.$, $\mathrm{p}$-value $\left.<0,0001\right)$. Elle est à peine plus intense en saison des pluies qu'en saison sèche (tab. 9.1).

Ce résultat est différent de celui de Compaoré (2006) sur un karité de $42 \mathrm{~cm}$ de diamètre dont la transpiration a été estimée à 79 1/jour en début de saison sèche, et presque la moitié (32 1/jour) en fin de saison sèche.

Par ailleurs, Bayala et al. (2008) ont estimé à 121 1/jour la transpiration moyenne annuelle d'un arbre de $64 \mathrm{~cm}$ de diamètre situé dans un parc cultivé. Ce résultat est cohérent avec le sens de la relation que nous avons obtenue entre la transpiration et le diamètre du tronc. Néanmoins, la valeur de la transpiration est supérieure à celle prédite par la relation obtenue dans notre étude pour un arbre de ce diamètre. Situés dans un parc agroforestier, les karités instrumentés par Bayala et al. (2008) sont soumis à une compétition pour les ressources probablement moins intense que dans la jachère que nous avons étudiée. En effet, dans la plupart des cas, la densité des arbres dans les parcs est plus faible qu'en jachère (Picasso, 1984). De plus, les arbres des parcs ont pu bénéficier des amendements apportés par les paysans par l'intermédiaire de la culture associée. Ces arbres sont ainsi plus vigoureux. 


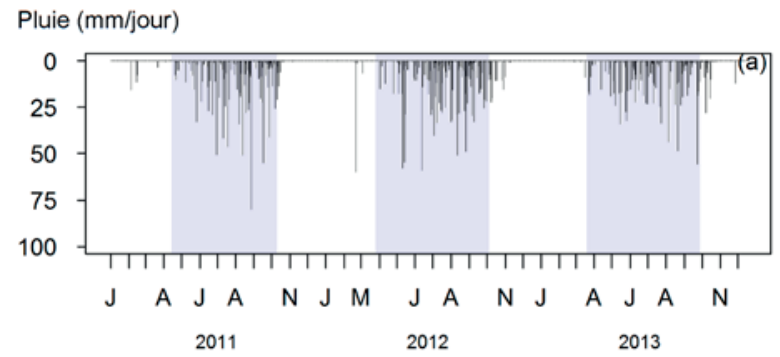

Figure 9.3a. Variation saisonnière de la pluviométrie

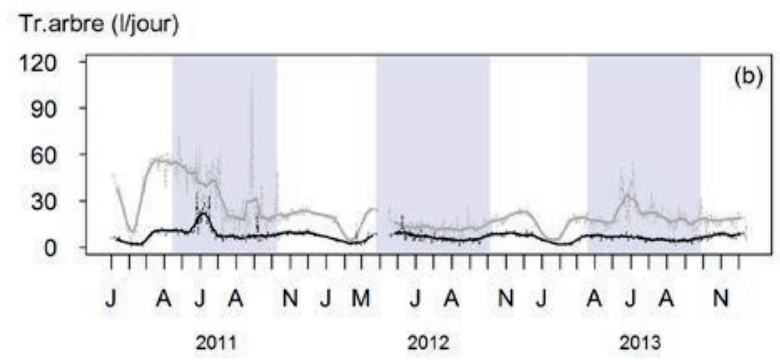

Figure 9.3b. Variation saisonnière de la transpiration de l'arbre de karité Vitellaria paradoxa 1 (V.p1, gris) et V.p2 (noir).

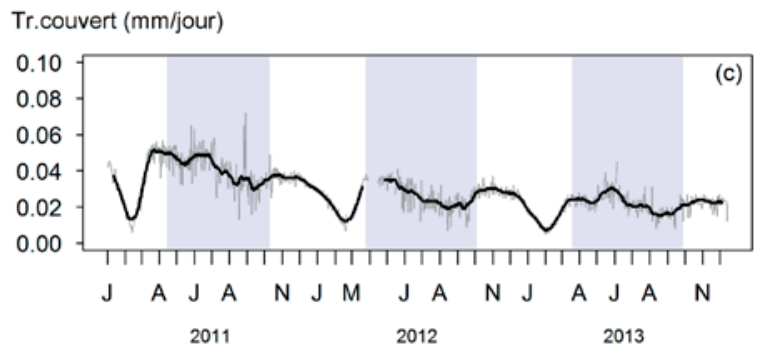

Figure 9.3c. Variation saisonnière de la transpiration de la population de karité (Tr couvert).

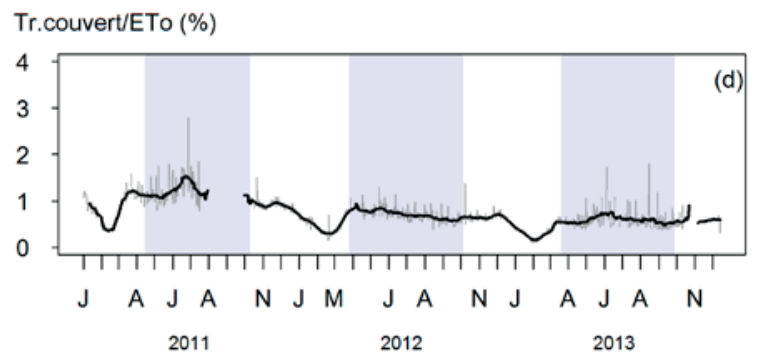

Figure 9.3d. Variation saisonnière du ratio Tr couvert / Eto (\%).

Figure 9.3. Variation saisonnière sur les trois années (2011-2013) d'étude dans la jachère de Bira au nord-ouest du Bénin. Les bandes verticales délimitent les saisons des pluies; b-d : données journalières (ligne fine) et moyenne glissante sur 21 jours (ligne épaisse). 


\section{L'intensité de la transpiration à l'échelle de la population de karités}

Lintensité de la transpiration à l'échelle de la population de karités a été estimée au cours des premiers mois de l'installation (2011), afin qu'elle ne soit pas affectée par les phénomènes de cicatrisation. Elle varie de 0,01 à $0,07 \mathrm{~mm} / \mathrm{jour}$, avec une moyenne de 0,028 ( $\pm 0,011) \mathrm{mm} /$ jour (fig. 9.3c et tab. 9.1). Elle est significativement plus élevée en saison des pluies qu'en saison sèche $(\mathrm{t}$ - test $; \mathrm{t}=4,28 ; \mathrm{p}<0,0001)$, bien que faiblement; comme l'est la transpiration moyenne journalière d'un arbre (tab. 9.1). La faible différence observée entre les deux saisons suggère que la disponibilité en eau du sol n'est jamais limitante au cours de l'année. Plusieurs mécanismes intrinsèques au karité tels que le stock interne d'eau du xylème, l'anatomie et la physiologie du xylème pourraient également contribuer à expliquer la relative stabilité de sa transpiration (Eamus et al., 2000).

Les valeurs de la transpiration de la population de karités en 2011 correspondent à 0,42 à 1,32\% de la demande atmosphérique Eto (fig. 9.3c et fig. 9.3d). Au cours des trois années d'étude et en dehors des périodes de renouvellement des feuilles, la variation du rapport $\mathrm{Tr} /$ Eto reste également faible ( $\mathrm{Tr}$ est la transpiration de la population de karités sur la parcelle), y compris en saison sèche (avril 2011, novembre-décembre 2011 et 2012). Cette stabilité confirme que la transpiration est principalement sous le contrôle de la demande atmosphérique, pas vraiment sous celui de la disponibilité en eau du sol. Au cours d'une année, la transpiration de la population de karités représente seulement $1,15 \%$ de la pluie annuelle. D'après des études menées en station de recherche agronomique, Ong et Leakey (1999) indiquent que la transpiration peut représenter 30 à $45 \%$ des précipitations annuelles dans un système agroforestier bien géré.

\section{Conclusion}

À partir du couvert de la jachère que nous avons étudiée, la marge d'augmentation de la densité de la population de karité est potentiellement élevée. Compte tenu des pratiques agricoles encore extensives en Afrique soudano-sahélienne, la culture associée ne risque pas de consommer l'eau à hauteur des proportions indiquées par Ong et Leakey (1999). Ainsi, même multipliée par trois, la densité des karités (17 arbres/ha) aurait probablement peu d'impact sur la consommation en eau de l'ensemble de la couverture végétale à l'échelle de la parcelle. Cependant, l'augmentation de la densité des arbres conduit à limiter la production de la culture associée par la diminution de son accès à la lumière à cause du feuillage des arbres. C'est ce compromis qui doit être optimisé pour une intensification durable de la production des parcs à karité. La consommation en eau des cultures annuelles en parc et des graminées spontanées en jachère n’a pas été abordée dans notre étude, ni dans cet ouvrage. En revanche, l'effet du karité en parc sur la fertilité du sol (carbone et nutriments) et sur le rendement de la culture associée est abordé dans le chapitre 10. 


\section{- Bibliographie}

Allen R.G., Pereira L.S., Raes D., Smith M., 1998. Crop evapotranspiration-guidelines for computing crop water requirements. FAO irrigation and drainage paper 56. Rome: FAO.

Awessou K.G.B., Peugeot C., Rocheteau A., Seguis L., Do F.C., Galle S., Bellanger M., Agbossou E., Seghieri J., 2017. Differences in transpiration between a forest and an agroforestry tree species in the Sudanian belt. Agroforestry systems, 91: 403-413. Doi: 10.1007/s10457-016-9937-8.

Bayala J, Ouédraogo S.J., Ong C.K., 2009. Early growth performance and water use of planted West African provenances of Vitellaria paradoxa C.F. Gaertn (karité) in Gonsé, Burkina Faso. Agroforestry systems, 75: 117-127.

Bayala J., Heng L.K, van Noordwijkc M., Ouedraogo S.J., 2008. Hydraulic redistribution study in two native tree species of agroforestry parklands of West African dry savanna. Acta oecologica, 34: 370-378.

Bodart C, Brink A.B., Donnay F., Lupi A., Mayaux P., Achard F., 2013. Continental estimates of forest cover and forest cover changes in the dry ecosystems of Africa between 1990 and 2000. Journal of biogeography, 40: 1036-1047.

Chevalier A., 1946. Larbre à beurre d'Afrique et l'avenir de sa culture. Oléagineux, 1: 7-1.

Compaoré H., 2006. The impact of savannah vegetation on the spatial and temporal variation of the actual evapotranspiration in the Volta Basin, Navrongo, Upper East Ghana. Ecology and development series, 36: 1-155.

Do F., Rocheteau A., 2002. Influence of natural temperature gradients on measurements of xylem sap flow with thermal dissipation probes. 2. Advantages and calibration of non-continuous heating system. Tree physiology, 22: 649-654.

Eamus D., O'Grady A.P., Hutley L.B., 2000. Dry season conditions determine wet season water use in the wet-dry tropical savannas of northern Australia. Tree physiology, 20: 1219-1226.

Granier A., 1985. Une nouvelle méthode pour la mesure du flux de sève brute dans le tronc des arbres. Annals of forest science, 42: 193-200.

Isarangkool Na Ayutthaya S., Do F.C., Pannengpetch K., Junjittakarn J., Maeght J.L, Rocheteau A., Cochard H., 2010. Transient thermal dissipation method of xylem sap flow measurement: multispecies calibration and field evaluation. Tree physiology, 30: 139-148.

Issoufou H.B., Rambal S., Le Dantec V., O1“ M., Laurent J.P., Saadou M., Seghieri J., 2015. Is the WBE model appropriate for semi-arid shrubs subjected to clear cutting? Tree physiology. 35: 197-208.

Jiménez M.S., Nadezhdina N., Cermak J., Morales D., 2000. Radial variation in sap flow in five laurel forest tree species in Tenerife, Canary Islands. Tree physiology, 20: 1149-1156.

Kumagai T., Aoki S., Shimizu T., Otsuki K., 2007. Sap flow estimates of stand transpiration at two slope positions in a Japanese cedar forest watershed. Tree physiology, 27: 161-168.

Leroux L., 2012. Analyse diachronique de la dynamique paysagère sur le bassin supérieur de l'Ouémé (Bénin) à partir de l'imagerie Landsat et Modis : cas d'étude de la commune de Djougou. Rapport interne, projet ANR-Escape. https://www.locean-ipsl.upmc.fr/ ESCAPE/Livrables/ D1.3c.pdf, (consulté le 20 mars 2019).

Nadezhdina N., Cermák J., Ceulemans R., 2002. Radial patterns of sap flow in woody stems of dominant and understory species: scaling errors associated with positioning of sensors. Tree physiology, 22: 907-918.

Nikiéma A., 2005. Agroforestry parkland species diversity: uses and management in semi-arid West Africa (Burkina Faso). Thèse de doctorat, Wageningen University, Pays-Bas. ISBN : 90-8504-168-6.

Nikiéma A., Van Der Maesen L.J.G., Hall J.B., 2003. The impact of parkland management practices on plant resources diversity. In: Teklehaimanot Z. (éd.). Improved management of agroforestry parkland systems in Sub-Saharan Africa. EU/Inco Project Contract IC18-CT98-0261. Third annual progress report, Bangor, Royaume-Uni, University of Wales.

Ong C.K., Leakey R.R.B., 1999. Why tree-crop interactions in agroforestry appear at odds with tree-grass interactions in tropical savannahs. Agroforestry systems, 45: 109-129. 
Ong C., Black C.R., Wilson J., Muthuri C., Bayala J., Jackson N.A., 2014. Agroforestry: hydrological impacts. In: van Alfen N., (ed.) Encyclopaedia of agriculture and food systems, vol. 1. San Diego: Elsevier, 244-252.

Pataki D.E., Oren R., 2003. Species differences in stomatal control of water loss at the canopy scale in a mature bottomland deciduous forest. Advances in water resources, $26: 1267-1278$.

Picasso C., 1984. Synthèse des résultats acquis en matière de recherche sur le Karité au Burkina Faso de 1950 à 1958. Ivraz/Irho.

Seghieri J., 2019. Shea tree (Vitellaria paradoxa Gaertn. F.): from local constraints to multi-scale improvement of economic, agronomic and environmental performance in an endemic Sudanian multipurpose agroforestry species. Agroforestry Systems. https://doi.org/10.1007/s10457-019-00351-1.

Tateishi M., Kumagai T., Utsumi Y., Umebayashi T., Shiiba Y., Inoue K., Kaji K., Cho K., Otsuki K., 2008. Spatial variations in xylem sap flux density in evergreen oak trees with radial-porous wood: comparisons with anatomical observations. Trees, 22: 23-30.

Tia L., 2008. Modeling of vegetation dynamics and its contribution to the water balance in semiarid lands of West Africa. Ecology and development series, 58.

Venkatraman K. and Ashwath N., 2016. Transpiration in 15 tree species grown on a phytocapped landfill site. Hydrology current research, 7: 2.

West G.B., Brown J.H., Enquist B.J., 1999. A general model for the structure and allometry of plant vascular systems. Nature, 400: 664-667.

Wiedemann A., Jiménez S.M., Rebman C., Cuntz M., Herbst M., 2013. An empirical study of the wound effect on sap flux density measured with thermal dissipation probes. Tree physiology, 36 : $1471-1484$. 


\title{
Chapitre 10 \\ Impacts du karité sur les ressources du sol et la production d'une culture de maïs associée dans un parc agroforestier soudanien du Nord-Est du Bénin
}

\author{
Clermont-Dauphin C., Séguis L., Velluet C., Degbé M., \\ Cournac L. et Seghieri J.
}

\begin{abstract}
Résumé. Les impacts des karités (Vitellaria paradoxa) sur les contenus du sol en eau, en nutriments et en carbone, ainsi que sur la production associée de maïs pluvial ont été évalués dans un parc agroforestier au Nord-Est du Bénin. Pour ce faire, les résultats des mesures sous houpier et hors houppier ont été comparés. Le rendement de la culture s'est révélé inférieur sous le houppier, malgré des conditions de fertilité et d'humidité du sol qui restaient favorables. Notre hypothèse est que cet effet négatif serait causé par la limitation par le houppier du rayonnement incident sur la culture. De plus, on a observé une contribution significative des arbres à l'enrichissement de la matière organique du sol sur l'ensemble du parc agroforestier. Cet effet positif de la présence des arbres pourrait se traduire par un impact bénéfique sur le rendement du maïs. Pour le vérifier, les rendements obtenus dans les conditions de cette étude devront être comparés aux résultats obtenus dans une situation témoin (sans arbres), toutes choses égales par ailleurs.
\end{abstract}

\begin{abstract}
The impacts of shea trees (Vitellaria paradoxa) on soil water, nutrients and carbon contents, and on the yield of the associated rainfed maize cultivation are assessed in a shea parkland in the North-East Benin. Measurements were made under and out of the tree crown covers. Maize yield was found lower under the crown cover than out, despite favourable conditions of soil fertility and moisture. Our assumptiun is that this difference may be due to the limitation by the crown cover of the incoming radiation toward the maize crop. In addition, the trees were found to have a significant contribution to the soil organic matter over the whole parkland. This favourable impact of the trees may have a positive effect on the associated maize yield at the parkland scale. To check this assumptium, it will be necessary to compare our results on the maize yield to the one that will be obtained in a control situation (without trees), other things being equal.
\end{abstract}




\section{Introduction}

Depuis plusieurs siècles, les associations de karités (Vitellaria paradoxa) et de cultures annuelles sous forme de parcs agroforestiers dominent les surfaces cultivées de la bande soudanienne d'Afrique de l'Ouest. Ces arbres bénéficient de la protection des paysans en raison de l'intérêt économique local et international de leurs produits (Sallé et al., 1991; Serpantié, 1996 et 2000). Ces parcs agroforestiers retiennent également de plus en plus l'attention de la recherche scientifique dans le but de préciser leurs performances écologiques et, en particulier, d'estimer les moyens d'obtenir durablement sur ces parcelles agroforestières des rendements plus élevés que les cultures pures, comme cela a été rapporté pour d'autres systèmes agroforestiers (Rao et al., 1998; Ong et Leakey, 1999).

Quelques travaux concernent les systèmes à base de karités. Mais leurs résultats montrent des tendances parfois contradictoires en termes de performances agronomiques (Kater et al., 1992; Kessler, 1992; Rao et al., 1998; Boffa et al., 2000; Bayala et al., 2002; Bazié et al., 2012). Il est donc nécessaire de mieux connaître les interactions entre les différentes composantes du système sol/arbre/culture annuelle, en considérant chaque ressource susceptible d'être impactée par la présence de l'arbre : l'eau, les nutriments et le rayonnement. Les impacts positifs ou négatifs des karités sur ces ressources peuvent être d'ampleur variable et dépendent des conditions pédoclimatiques et des exigences des cultures associées. La quantification de ces différents impacts est donc nécessaire pour optimiser la densité des arbres et leurs modes de gestion en fonction des meilleurs compromis entre les services et les disservices écosystémiques rendus par les arbres.

Le but de cette étude (financée dans le cadre du projet Safse IRD-Cirad) est d'évaluer les effets d'un peuplement de karités géré suivant les pratiques paysannes locales sur le stock de carbone et d'eau dans le sol, le statut nutritionnel de l'azote et le rendement du maïs, l'une des deux céréales (avec le sorgho) les plus couramment cultivées dans les parcs à karités de la région.

Deux hypothèses seront testées :

- la proximité des arbres favorise l'accumulation des ressources du sol (eau, carbone et nutriments);

- l'accumulation de ces ressources a un effet positif sur le rendement de la culture associée.

\section{Matériels et méthodes}

\section{Zone d'étude et dispositif expérimental}

Le site d'étude est un parc à karités typique d'Afrique de l'Ouest. Il est situé dans le village de Gnogambi $\left(1.801270^{\circ} \mathrm{E}-9.741561^{\circ}\right)$, commune de Djougou au nord-est du Bénin. Le sol de type ferrugineux à texture sablo-limoneuse dans l'horizon 0-30 cm est dominant dans la région. Le climat est de type soudano-guinéen, avec une pluviométrie moyenne de $1200 \mathrm{~mm} /$ an sur la période 1950-2005 (Seghieri et al., 2009) concentrée en une seule saison des pluies d'avril à octobre. Le biotope d'origine du karité serait la 
savane arborée ouverte et la forêt claire dans lesquelles l'essence est dominée au moins en hauteur par d'autres essences (Serpantié et al., 1996). Le karité aurait été progressivement sélectionné au cours des soixante dernières années par les exploitants agricoles pour les produits (beurre) qu'ils en tirent. Aujourd'hui, les peuplements sont toujours maintenus à des densités telles qu'il n'y a pas de compétition pour les ressources avec les cultures associées. Dans le parc étudié, la distance entre arbres varie de 20 à $40 \mathrm{~m}$. Les densités maximales ne dépassent donc pas 25 arbres/ha. Bien que le karité soit l'espèce majoritaire, on y retrouve aussi quelques pieds de néré (Parkia biglobosa) comme dans la plupart des parcs à karités. La circonférence du tronc de karité varie de 60 à $150 \mathrm{~cm}$. En association avec le peuplement arboré, une culture annuelle vivrière pluviale mixte de maïs et de sorgho (en alternance dans chaque rang) est reconduite quasiment tous les ans. Après la récolte des céréales, les résidus de culture sont laissés sur place pour servir de fourrage pendant la saison sèche.

En 2014, le dispositif d'étude installé au début de la saison des pluies comprenait quatre pieds de karités. Cette taille d'échantillon a été retenue en référence par Bayala et al. (2016). Ils mettaient en évidence, avec un échantillon de même taille sur un parc à karités au Burkina Faso, un effet significatif de la distance à l'arbre sur les stocks de carbone des sols.

Les quatre arbres ont été classés en deux groupes en fonction de la circonférence des troncs :

- deux troncs étaient parmi les plus gros du parc $(147 \mathrm{~cm}$ et $113 \mathrm{~cm}$, circonférence mesurée à 1,30 m au-dessus du sol);

- deux troncs parmi les plus petits $(80 \mathrm{~cm}$ et $66 \mathrm{~cm})$.

Les observations ont été faites dans deux zones concentriques : sous le houppier à $3 \mathrm{~m}$ du tronc et hors houppier à $10 \mathrm{~m}$ du tronc.

\section{Observations et analyses effectuées}

Les mesures et les observations effectuées dans ces deux zones ont porté sur les éléments chimiques, le stock du carbone dans le sol et son abondance en isotopes stables ${ }^{13} \mathrm{C}$ et ${ }^{12} \mathrm{C}$, la succion en eau du sol, la teneur en azote d'un échantillon de pieds entiers de maïs au stade montaison, ainsi que sur le rendement grains et pailles du maïs et ses composantes à la récolte.

\section{Mesures des composantes chimiques du sol}

Des échantillons composites du sol ont été prélevés sous le houppier et hors houppier à la tarière aux quatre points cardinaux de chaque zone concentrique et sur deux horizons (0-10 cm et 10-30 cm). Les analyses chimiques ont été effectuées par le Laboratoire mixte international, Iesol au Sénégal sur la plateforme Lama ${ }^{1}$. Le carbone et l'azote totaux ont été dosés par oxydation catalytique avec un analyseur élémentaire CHN (Flash EA1112 série, Thermofinnigan, France). Le phosphore total a été extrait par un mélange d'acide nitrique et chlorhydrique concentrés, le phosphore assimilable a été extrait au bicarbonate de sodium à $\mathrm{pH}=8,5$ (méthode

1. http://lama.ird.sn/prestations/index.htm. 
Olsen), pour ces deux extractions, les ions phosphates libérés ont été dosés par colorimétrie (Dabin, 1967) à l'aide d'un analyseur en flux continu de type Technicon (Seal analytical, Royaume-Uni). Les bases échangeables ont été extraites à l'acétate d'ammonium et dosées par spectrophotométrie d'absorption atomique (Varian 220 FS).

Les mesures isotopiques ont été réalisées sur deux horizons (0-10 cm et 10-30 cm) exprimées sous la forme d'un rapport de l'isotope lourd $\left({ }^{13} \mathrm{C}\right)$ sur l'isotope léger $\left({ }^{12} \mathrm{C}\right)$. La signature ${ }^{13} \mathrm{C}$ de chaque échantillon de sol a été calculée comme la différence entre ce rapport (Réch) et celui d'un standard international (Rref) et exprimé en pour mille $(\% \circ)$ :

$$
{ }^{13} \mathrm{C}(\%)=(\text { Réch }- \text { Rref }) / \text { Rref } \times 1000
$$

La signature ${ }^{13} \mathrm{C}$ du sol a été utilisée comme indicateur de la contribution respective de l'arbre et de la culture annuelle au stock de carbone présent dans le sol. Cet indicateur s'appuie sur le fait que l'arbre ayant un fonctionnement photosynthétique en $\mathrm{C}_{3}$ a une signature ${ }^{13} \mathrm{C}$ plus basse que les céréales qui ont une photosynthèse en $\mathrm{C}_{4}$. En faisant l'hypothèse que la signature ne varie pas significativement avec la maturation de la matière organique, les proportions de carbone de chaque origine ont été calculées comme proposé par Balesdent et al. (1988) :

$$
\begin{aligned}
& \mathrm{C}_{\mathrm{T}}=\left({ }^{13} \mathrm{C}_{\mathrm{t}}-{ }^{13} \mathrm{C}_{\mathrm{C}}\right) /\left({ }^{13} \mathrm{C}_{\mathrm{T}}-{ }^{13} \mathrm{C}_{\mathrm{C}}\right) ; \\
& \mathrm{C}_{\mathrm{C}}=\left({ }^{13} \mathrm{C}_{\mathrm{t}}-{ }^{13} \mathrm{C}_{\mathrm{T}}\right) /\left({ }^{13} \mathrm{C}_{\mathrm{C}}-{ }^{13} \mathrm{C}_{\mathrm{T}}\right) .
\end{aligned}
$$

$\mathrm{C}_{\mathrm{T}}$ et $\mathrm{C}_{\mathrm{C}}$ sont respectivement les proportions de carbone du sol provenant de l'arbre (T) et de la culture $(\mathrm{C}) ;{ }^{13} \mathrm{C}_{\mathrm{T}}$, signature isotopique de la biomasse de l'arbre; ${ }^{13} \mathrm{C}_{\mathrm{C}}$, signature isotopique de la biomasse de la culture; ${ }^{13} \mathrm{C}_{\mathrm{t}}$, signature isotopique du $\mathrm{C}$ du sol. Ces données ont été déterminées au laboratoire de biochimie et physiologie moléculaire des plantes de l'Inra à Montpellier.

\section{Mesure de la dynamique de l'eau dans le sol}

Les succions en eau du sol sous le houppier et hors houppier ont été déterminées à partir d'un suivi tensiométrique entrepris d'avril 2014 à août 2015 au voisinage d'un des plus gros karités du champ (KG1). Deux forages de 4,5 m de profondeur et $6,5 \mathrm{~cm}$ de diamètre ont été réalisés (à l'aide d'une foreuse Stenuick pneumatique avec marteau fond de trou). Le premier forage est situé au voisinage du tronc, le second en dehors du houppier. Dans chaque forage, des tensiomètres automatiques (Campbell 229 Heat dissipation matric water potential sensor) ont été implantés à 10 profondeurs : 0,$25 ; 0,50 ; 0,75 ; 1 ; 1,5 ; 2 ; 2,5 ; 3 ; 3,5 ; 4$ m. Les données tensiométriques permettent de suivre les phases d'humectation-dessèchement du profil et de calculer les gradients de charge verticaux indicateurs de la direction ascendante ou descendante des flux hydriques. À proximité des profils tensiométriques, deux tubes d'accès de sonde à neutrons ont été implantés afin de mesurer les profils de la teneur en eau du sol. Un suivi neutronique a été réalisé de juin à fin octobre 2014.

\section{Mesures sur la culture de maïs}

Le rendement en grains et en pailles du maïs et ses composantes à la récolte ont été mesurés sur une surface de $2 \times 3 \mathrm{~m}$ sous le houppier et hors houppier (A et C). Les poids de grains et de pailles ont été obtenus après séchage au soleil. 
Les teneurs en azote $(\mathrm{N})$ ont été mesurées sur un échantillon de pieds entiers de maïs au stade montaison déplantés sur une placette de $2 \times 3 \mathrm{~m}$ au niveau de chaque zone (A et C). Ces analyses ont également été réalisées sur la plateforme Lama par la méthode Kjeldahl pour l'azote, par absorption atomique pour les cations et par colorimétrie au Technicon après extraction à l'eau régale pour le phosphore total.

Les résultats ont permis de calculer l'indice de nutrition azotée de la culture au stade montaison. Lindice de nutrition azotée (INN) est le rapport de la teneur en azote mesurée sur la teneur en azote minimale nécessaire pour une croissance maximale. La nutrition azotée de la plante varie comme la valeur de l'INN. Une valeur INN = 1 indique que la nutrition azotée de la plante est optimale, alors qu'une valeur inférieure à 1 indique que la nutrition azotée est limitante pour la croissance de la culture. La teneur en azote minimale nécessaire pour une croissance optimale a été calculée à partir de l'équation proposée par Lemaire et Gastal (1997) en l'absence de facteur limitant :

$\mathrm{N} \%=5,06(\mathrm{~W})^{-0,106}$

où $\mathrm{N}$ est la teneur en azote de l'échantillon et $\mathrm{W}$ (en grammes) la biomasse végétative moyenne d'un pied à la date d'analyse.

\section{Analyses statistiques}

Les caractéristiques chimiques des sols ont été évaluées par analyse de variance (Anova) intégrant les effets de trois facteurs : localisation dans l'horizon 0-10 ou 10-30 cm; la circonférence du tronc, petit ou gros; la distance du sol analysé au tronc du karité, 3 ou $10 \mathrm{~m}$. Les interactions de ces facteurs pris deux à deux ont été également analysées.

Les rendements et les composantes du rendement du maïs ont été analysés par des analyses de variance intégrant les effets des facteurs «distance au tronc de l'arbre» et «circonférence du tronc» de l'arbre. Ces analyses ont été effectuées avec le logiciel Statistica.

\section{W Résultats et discussions}

\section{Complexité des effets de l'arbre sur les caractéristiques physico- chimiques du sol}

L'horizon 0-30 cm de sol se caractérise par une texture sablo-limoneuse (14\% de limon, $18 \%$ de sable, $58 \%$ de sable grossier et $9 \%$ d'argile), une densité apparente de 1,76, un $\mathrm{pH}$ moyen de 5, ainsi qu'une CEC (capacité d'échange cationique) moyenne de 10 méq/100g. Les analyses de variances réalisées sur les caractéristiques chimiques du sol des horizons 0-10 et 10-30 montrent :

- un effet significatif de l'horizon de sol (0-10 ou 10-30 cm) sur les teneurs en N, C et P totaux, ainsi que sur P Olsen et la CEC. L'horizon 0-10 cm est généralement plus riche en matière organique et en éléments minéraux que l'horizon 10-30 cm (tab. 10.1). Ces résultats sont cohérents avec le fait que la matière organique est apportée essentiel- 


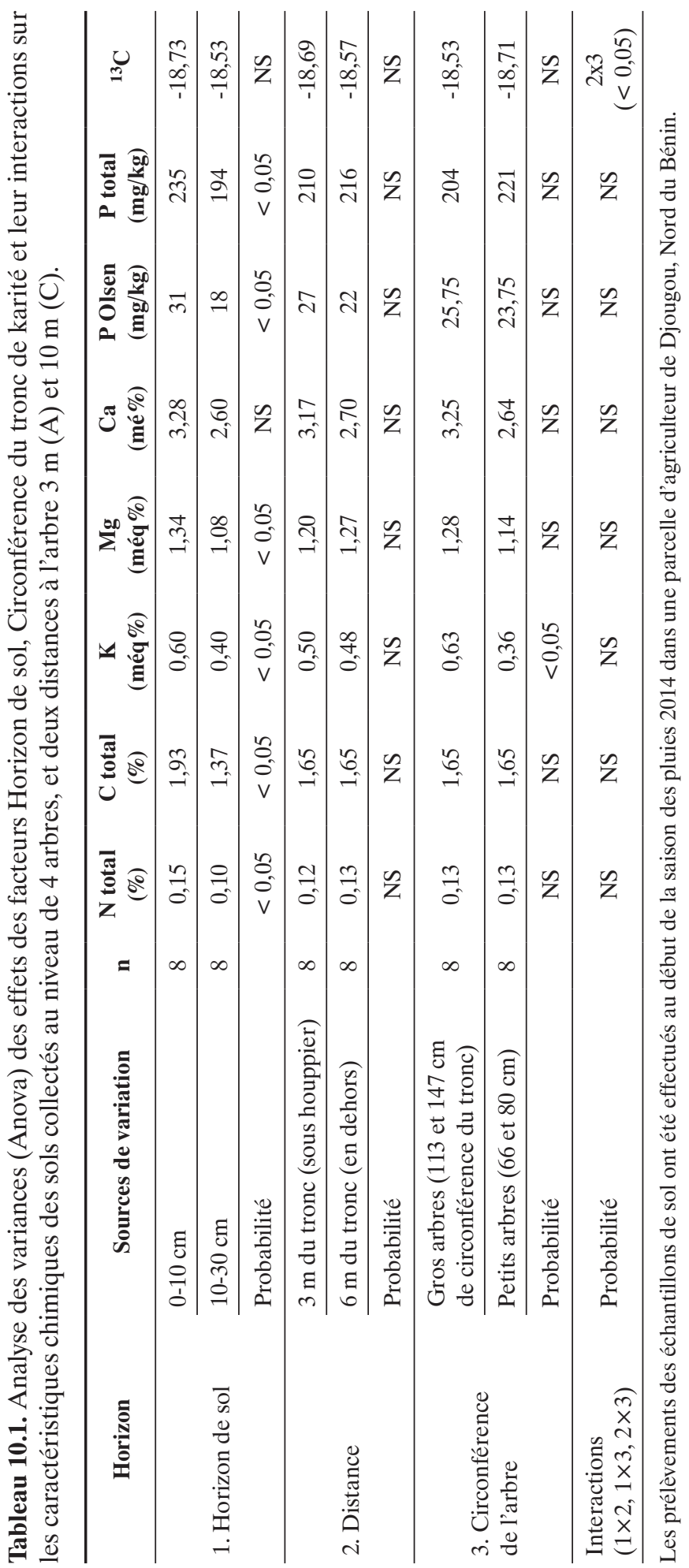


lement en surface et que le travail du sol à la houe reste très superficiel et n'entraîne donc pas un enfouissement important des matières organiques. Ces résultats sont conformes à ceux de Bayala et al. (2006) sur ces systèmes agroforestiers;

- un effet non significatif de la distance du prélèvement au tronc de l'arbre.

La signature $\delta^{13} \mathrm{C}$ des litières d'arbres s'élève en moyenne à $-29 \%$, celle du maïs à $-12 \%$. Le fumier des bovins présents sur les parcelles a une signature ${ }^{13} \mathrm{C}$ de $-19 \%$, donc intermédiaire. Le $\delta^{13} \mathrm{C}$ des sols s'élève en moyenne à $-18 \%$ et ne varie pas significativement en fonction de la distance à l'arbre (tab. 10.1). La contribution de la litière de l'arbre au stock de carbone du sol dans l'horizon 0-10 cm s'élève en moyenne à $40 \%$ du stock total. L'hypothèse selon laquelle l'arbre favorise l'accumulation de carbone dans le sol semble donc se confirmer.

Les analyses de variance montrent cependant une interaction significative des facteurs «circonférence du tronc» et «distance au tronc de l'arbre» :

- pour les petits arbres, la signature $\delta^{13} \mathrm{C}$ du sol devient moins négative quand on s'éloigne de l'arbre, indiquant ainsi une contribution de l'arbre au stock de carbone du sol plus importante sous le houppier qu'en-dehors (fig. 10.1b);

- pour les gros arbres, l'effet de la distance au tronc s'inverse ou s'annule (fig. 10.1a). En effet, la matière organique du sol sous le houppier paraît avoir la même signature que celle de la moyenne du parc. Sur un des deux pieds où cette mesure est effectuée, elle montre même, de manière apparemment paradoxale, une contribution de l'arbre moins importante sous son houppier que quand on s'en éloigne.

Nos résultats sont cohérents avec ceux de Picasso (1984) qui indique un effet favorable des grands arbres à des distances de plus de $15 \mathrm{~m}$ de leur tronc, en lien avec une dispersion aléatoire de leur litière. D'autres causes peuvent être évoquées pour expliquer les différences entre les petits et les gros arbres, mais elles restent à vérifier. Par exemple, l'hétérogénéité de la fertilisation organique à l'intérieur du parc agroforestier en est une, avec notamment la répartition du fumier du bétail, car les animaux se réfugient préférentiellement à l'ombre des gros arbres en saison sèche.

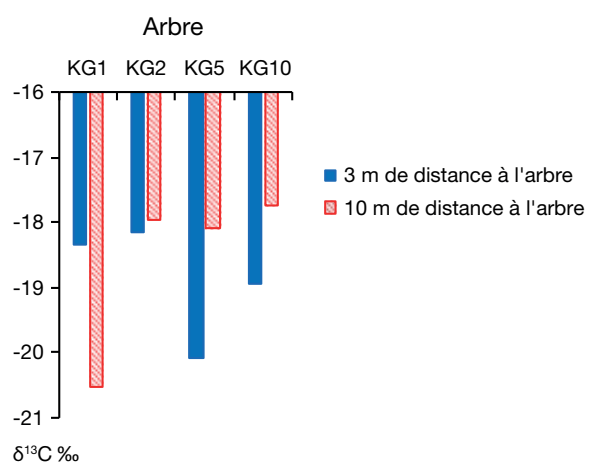

Figure 10.1a. Abondance naturelle en ${ }^{13} \mathrm{C}$ $\left(\delta^{13} \mathrm{C}\right.$ exprimé en $\%$ ) de la couche $0-10 \mathrm{~cm}$ de sol.

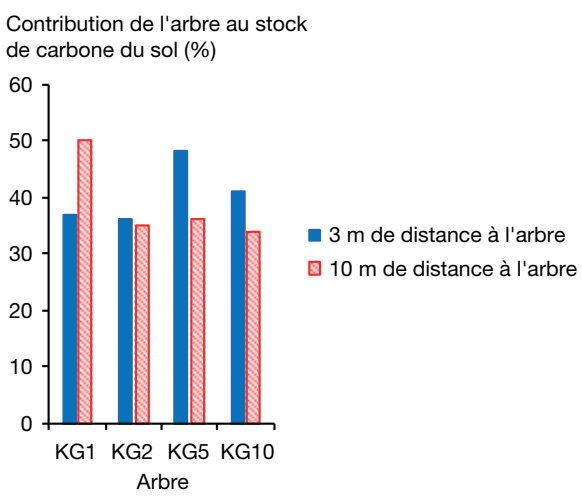

Figure 10.1b. Contribution de l'arbre au stock de carbone du sol (en \%).

Distances de $3 \mathrm{~m}$ et $10 \mathrm{~m}$ de 4 arbres de karité selon leur circonférence : KG1, $147 \mathrm{~cm}$; KG2, $113 \mathrm{~cm}$; $\mathrm{KG} 5,80 \mathrm{~cm}$; KG10, $66 \mathrm{~cm}$. 
Ici, la possibilité d'une répartition hétérogène de la matière organique stable du sol antérieure à la mise en culture et masquant un éventuel effet de la distance aux deux grands arbres échantillonnés est à tester également. Enfin, des variations microtopographiques définissant des points d'accumulation préférentielle de la litière des arbres pourraient favoriser des contributions localement élevées de la biomasse arborée au carbone du sol à une certaine distance du tronc. Ces différentes hypothèses demandent à être testées dans des travaux ultérieurs.

\section{Effet des arbres sur les flux d'eau}

\section{En saison des pluies}

À proximité du seul arbre instrumenté, les mesures tensiométriques au cours de chaque saison des pluies de 2014 et 2015 indiquent une humidification plus tardive en profondeur (1,5 m et plus) et une teneur en eau moyenne moins élevée sous le houppier qu'en dehors du houppier (fig. 10.2).

Trois hypothèses sont avancées :

- la nature du sous-sol. Lanalyse visuelle des échantillons indique dans l'horizon 2 à $4 \mathrm{~m}$ de profondeur un sol un peu moins argileux sous le houppier qu'en dehors du houppier, il est semblable plus en profondeur. Les mesures d'humidité neutronique (non présentées ici) montrent de manière homogène que jusqu'à $9 \mathrm{~m}$ de profondeur, le sol est moins humide sous le houppier qu'en dehors du houppier. Il ne semble donc pas possible d'attribuer les différences d'humidité observées à des différences pédologiques, même faibles;

- l'interception du feuillage. D'après les mesures, l'humidité superficielle associée aux premières pluies des deux saisons des pluies étudiées est plus prononcée sous le houppier qu'en dehors du houppier (fig. 10.2). Le houppier semblerait donc avoir pour effet de concentrer l'eau de pluie;

- la reprise transpiratoire de l'arbre. L'hypothèse la plus vraisemblable est donc que la diminution de l'humidité sous l'arbre, à partir de $2 \mathrm{~m}$ de profondeur, est attribuable à la reprise transpiratoire de l'arbre. Cette hypothèse est confirmée par les gradients de flux déduits des potentiels matriciels mesurés. Leur sens (gradient positif) indique une reprise de l'évapotranspiration dans l'horizon 2-4 m, sous l'arbre, d'avril à septembre 2014. En raison d'une défaillance matérielle des sondes, il n'y a pas de données disponibles en 2015.

En Afrique de l'Ouest, la littérature ne décrit pas de dispositif enregistrant le potentiel de l'eau dans le sol sur une aussi grande profondeur et pendant une aussi longue période que dans notre étude. Bayala et al. (2008a) ont placé des tensiomètres à proximité des karités dans l'horizon $0-1 \mathrm{~m}$ du sol pendant 3 mois après la saison des pluies : ils observent également des succions, bien que légèrement plus faibles que celles relevées dans notre étude.

\section{En saison sèche}

En saison sèche, les horizons superficiels du sol sont plus secs hors houppier que sous le houppier en comparaison de la saison des pluies (décembre 2014 à 

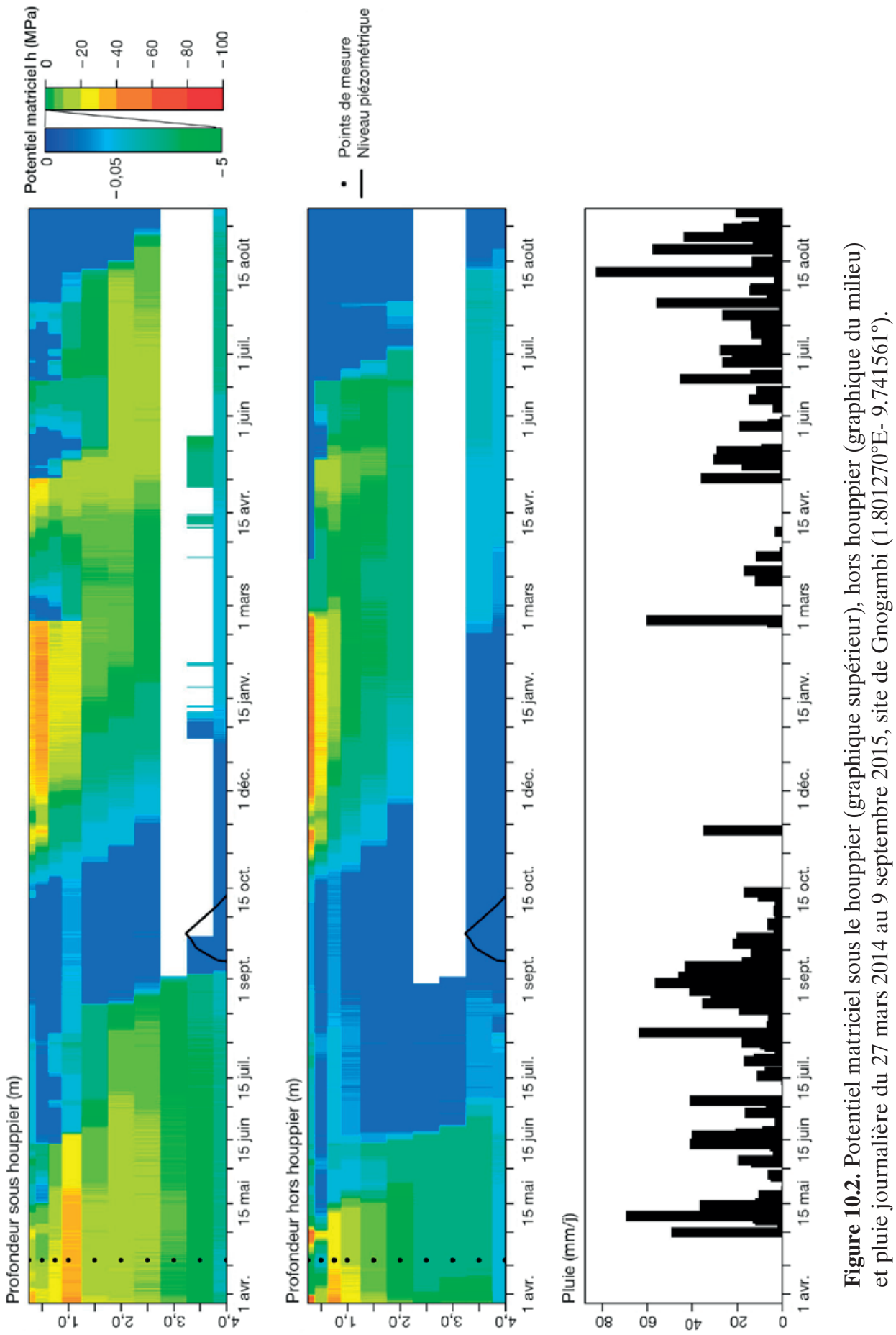
mi-mars 2015). Ce résultat confirme l'hypothèse d'un effet tampon du houppier sur les variations du microclimat (température et rayonnement incident). Par conséquent, il y a une limitation de l'évaporation des horizons superficiels du sol aux périodes chaudes et sèches grâce à une température et un rayonnement incident plus faibles sous le houppier.

\section{Conséquences sur la culture associée}

En termes de services écosystémiques, en concentrant les pluies, l'arbre est susceptible de favoriser le démarrage de la croissance de la culture sous son houppier en début de saison des pluies, quand les ressources en eau ne sont pas encore reconstituées. L'effet de la reprise transpiratoire sur la baisse de l'humidité au-delà de $2 \mathrm{~m}$ de profondeur ne devrait pas avoir d'impact sur la culture associée, dont la zone racinaire est généralement plus superficielle (observation personnelle). Cependant, les relations arbre-culture sont multiples et complexes, comme le montrent les résultats complémentaires ci-dessous.

\section{Élaboration du rendement du mais en fonction de la distance à l'arbre}

Des différences significatives entre les deux positions considérées (hors houppier, sous houppier) apparaissent dès le début de la montaison dans la biomasse végétale de la culture de maïs. Celle-ci est de $60 \%$ plus faible sous le houppier $(0,14 \mathrm{t} / \mathrm{ha})$ (tab. 10.2) que hors du houppier (0,37t/ha). Les différences de rendement en grains du maïs vont dans le même sens avec $2,2 \mathrm{t} /$ ha hors houppier et $1 \mathrm{t} /$ ha sous le houppier. Nos résultats ne montrent pas d'effet significatif de la circonférence de l'arbre sur ces deux variables.

Les relations entre le rendement en grains et le nombre de grains montrent que les différences de rendement en grains sont essentiellement liées aux nombres de grains plutôt qu'à leur poids qui reste stable entre les deux positions (différence non significative, tab. 10.2). Par conséquent, on peut en déduire que les différences de rendements ont leur origine avant ou pendant la période de floraison.

Découvrir les facteurs et processus en cause nécessite donc d'examiner cette phénophase en priorité. Les teneurs en azote $(\mathrm{N})$ des plantes de maïs entières au stade de montaison s'élèvent à 2,48 sous le houppier et $2,34 \%$ hors houppier ( $\mathrm{SE}=0,14$; $\mathrm{n}=4 ; \operatorname{Pr}>0,05)$. Elles correspondent à un indice de nutrition azotée de $60 \%$ en moyenne. Les risques de manque d'eau étant faibles dans les horizons de surface sous le houppier, comme nos résultats l'ont montré ci-dessus, la réduction du rayonnement incident déjà évoquée pour expliquer une moindre évaporation en saison sèche pourrait également être limitante des rendements du maïs. Cette hypothèse est largement partagée dans la littérature (Picasso, 1984; Kater et al., 1992; Kessler, 1992; Serpantié, 1996; Rao et al., 1998; Boffa et al., 2000; Bayala et al., 2002; Bazié et al., 2012; Saïdou et al., 2012).

Nos observations et résultats montrent que le houppier a des impacts favorables sur les ressources du sol (eau, carbone, azote) dans la zone racinaire des céréales, et des impacts défavorables sur le rendement de la céréale associée, très probablement à 
Tableau 10.2. Rendement en grains et en pailles et composantes du rendement du maïs sous le houppier et hors houppier du karité.

\begin{tabular}{lccc}
\hline & $\begin{array}{c}\text { En dehors } \\
\text { du houppier }\end{array}$ & $\begin{array}{c}\text { Sous } \\
\text { le houppier }\end{array}$ & $\begin{array}{c}\text { Degré de signification } \\
\text { de la différence }\end{array}$ \\
\hline Rendement $(\mathrm{t} / \mathrm{ha})$ & 2,20 & 1,05 & $\mathrm{~S}$ \\
\hline Nombre de pieds/m² & 3 & 2 & $\mathrm{NS}$ \\
\hline $\begin{array}{l}\text { Biomasse végétale } \\
\text { début montaison }(\mathrm{t} / \mathrm{ha})\end{array}$ & 0,37 & 0,14 & $\mathrm{~S}$ \\
\hline Biomasse végétale floraison $(\mathrm{t} / \mathrm{ha})$ & 2,27 & 1,18 & $\mathrm{~S}$ \\
\hline Nombre grains/épi & 281 & 168 & $\mathrm{~S}$ \\
\hline Nombre de grains/m & & 105 & $\mathrm{~S}$ \\
\hline Poids de 1000 grains $(\mathrm{g})$ & 220 & 105 & $\mathrm{NS}$ \\
\hline
\end{tabular}

Chaque valeur est la moyenne de quatre répétitions. Degré de signification de la différence : non significatif-NS (p-value $>0,05$ ), Significatif - S (p-value $<0,05$ ).

cause d'une réduction trop intense du rayonnement incident (non mesuré dans cette étude). Un compromis entre ces impacts pourrait être recherché à l'aide de techniques de gestion adaptées. Par exemple, l'élagage des houppiers selon une certaine forme, l'ajustement des densités d'arbres et leur répartition sur la parcelle cultivée (Picasso, 1984; Bayala et al., 2003 et 2008b) ou encore la sélection de variétés de céréales moins exigeantes en lumière sont des voies de recherches intéressantes.

Limpact des arbres-karités sur la production céréalière à l'échelle du parc résulterait alors du compromis entre les effets du houppier concentrés autour des arbres (effets négatifs sur le rayonnement, positifs sur l'eau) et les effets fertilisants de la litière des arbres dispersée sur l'ensemble du parc agroforestier. Pour tester cette dernière hypothèse, la comparaison avec une situation témoin (sans arbres) servant de référence serait nécessaire, toutes choses égales par ailleurs.

\section{" Conclusion}

Nos résultats ont montré que :

- l'aire d'influence du karité varie en fonction des indicateurs considérés;

- la présence des karités a des effets positifs ou négatifs en fonction des ressources considérées;

- le rayonnement incident intercepté semble le facteur limitant le plus probable de l'élaboration du rendement des plants de la céréale associée situés sous le houppier. Cette hypothèse reste à vérifier en estimant le degré de signification des différences entre les valeurs de ce rayonnement mesuré sous le houppier et hors houppier.

La mise en œuvre de recherches appliquées pour optimiser les compromis entre les impacts positifs et négatifs de la présence des karités implique de tester différents modes de gestion des arbres et des cultures associées dans le cadre d'un réseau d'expérimentations couvrant l'ensemble du gradient pédoclimatique de l'aire de répartition du karité. 


\section{" Bibliographie}

Balesdent J., Wagner G.H., Mariotti A., 1988. Soil organic matter turnover in long-term field experiments as revealed by carbon-13 natural abundance. Soil science society of american journal, 52: 118-124.

Bayala J., Balesdent J., Marol C., Zapata F., Teklehaimanot Z., Ouedraogo S.J., 2006. Relative contribution of trees and crops to soil carbon content in a parkland system in Burkina Faso using variations in natural ${ }^{13} \mathrm{C}$ abundance. Nutrient cycling in agroecosystems, 76: 193-201.

Bayala J., Heng L.K., van Noordwijk M., Ouedraogo S.J., 2008a. Hydraulic lift study in two native tree species of agroforestry parklands of West African dry savanna. Acta oncologica, 34: 370-378.

Bayala J., Mando A., Ouédraogo S.J., Teklehaimanot Z., 2003. Managing Parkia biglobosa and Vitellaria paradoxa prunings for crop production and improved soil properties in the Sub-Sudanian zone of Burkina Faso. Arid land research and management, 17: 283-296.

Bayala J., Ouedraogo S.J., Teklehaimanot Z., 2008b. Rejuvenating indigenous trees in agroforestry parkland systems for better fruit production using crown pruning. Agroforestry systems, 72: 187-194.

Bayala J., Teklehaimanot Z., Ouédraogo S.J., 2002. Millet production under pruned tree crowns in a parkland system in Burkina Faso. Agroforestry systems, 54: 203-214.

Bazié H.R., Bayala J., Zombré G., Sanou J., Ilstedt U., 2012. Separating competition related factors limiting crop performance in an agroforestry parkland system in Burkina Faso. Agroforestry systems, 84: 377-388.

Boffa J.M., Taonda S.J.B., Dickey J.B., Knudson D.M., 2000. Field-scale influence of karité (Vitellaria paradoxa) on sorghum production in the Sudan zone of Burkina Faso. Agroforestry systems, 49: 153-175.

Dabin B., 1967. Application des dosages automatiques à l'analyse des sols. 3. Cahiers de l'Orstom, série pédologie, 5 : 257-286

Kater L.J., Kante S., Budelman A., 1992. Karité (Vitellaria paradoxa) and néré (Parkia biglobosa) associated with crops in South Mali. Agroforestry systems, 18: 89-105.

Kessler J.J., 1992. The influence of karité (Vitellaria paradoxa) and néré (Parkia biglobosa) trees on sorghum production in Burkina Faso. Agroforestry systems, 17: 97-118.

Lemaire G., Gastal F., 1997. N uptake and distribution in plant canopies. In: Diagnosis of the nitrogen status in crops. G. Lemaire (ed.). Berlin: Springer Heidelberg. 3-43.

Ong C.K., Leakey, R.R.B., 1999. Why tree-crop interactions in agroforestry appear at odds with tree-grass interactions in tropical savannahs. Agroforestry systems, 45: 109-129.

Picasso C., 1984. Synthèse des résultats acquis en matière de recherche sur le karité au Burkina Faso de 1950 à 1958. Rapport de l'Institut de Recherche pour les Huiles et les Oléagineux. Paris : Irho-Cirad.

Rao M.R., Nair P.K.R., Ong C.K., 1998. Biophysical interactions in tropical agroforestry systems. Agroforestry systems, 38: 3-50.

Saïdou A., Balogoun I., Kone B., Gnangle C.P., Aho N., 2012. Effet d'un système agroforestier à karité (Vitellaria paradoxa C.F. Gaertn) sur le sol et le potentiel de production du maïs (Zea maize) en zone Soudanienne du Bénin. International journal of biological and chemical sciences, 6 : 2066-2082.

Sallé G., Boussim J., Raynald Roques A. et al., 1991. Le karité : une richesse potentielle. Perspectives de recherche pour améliorer sa production. Bois et forêts des tropiques, 228 : 11-23.

Seghieri J., Vescovo A., Padel K., Soubie R., Arjounin M., Boulain N., de Rosnay P., Galle S., Gosset M., Mouctar A.H., Peugeot C., Timouk F., 2009. Relationships between climate, soil moisture and phenology of the woody cover in two sites located along the West African latitudinal gradient. Journal of hydrology, 375(1-2) : 78-89.

Serpantié G., 1996. La production de karité (Butyrospermum paradoxum Gaertn. F. Hepper) des parcs arborés de l'ouest du Burkina Faso. Effets de différents modes de gestion. In : Floret C. (ed.) actes du colloque «La jachère, lieu de production", Bobo Dioulasso, Burkina Faso, 2-4 octobre 1996, Coraf, Union européenne, CNRST, Orstom, Ronéo, p. 73-80. 
Serpantié G., Bayala J., Helmfrid S., Lamien N., 1996. Pratiques et enjeux de la culture du karité (Butyrospermum paradoxum Gaertn. F. Hepper) dans l'Ouest du Burkina Faso. In: Floret C. (ed.) actes du colloque «La jachère, lieu de production», Bobo Dioulasso, Burkina Faso, 2-4 octobre 1996, 59-72.

Serpantié G., 2000. Artificialisation de deux ressources en zone soudanienne. In: Gillon Y., Chaboud C., Boutrais J., Mullon C., Weber J., (eds). Du bon usage des ressources renouvelables. Paris : Éditions IRD, 125-143. 



\section{B - Les parcs arbustifs}





\title{
Chapitre 11 \\ La régénération naturelle assistée dans le bassin arachidier du Sénégal, une alternative pour réduire la pauvreté en milieu rural
}

\author{
Sanogo D., Camara Baba A., Diatta Y., Coly L., Diop M., \\ BADJI M. ET BINAM J.-N.
}

Résumé. La régénération naturelle assistée est une pratique d'aide à la restauration du couvert ligneux utile aux populations locales au Sénégal, comme dans toute l'Afrique de l'Ouest. Les espèces ligneuses conservées dans les champs fournissent une large gamme de biens et de services aux hommes et aux femmes exploitants. Cette étude vise à évaluer les avantages socio-économiques que les ménages agricoles tirent de la régénération naturelle assistée dans deux zones agro-climatiques du Sénégal : le centre-nord et le centre-sud bassin arachidier du Sénégal. De plus, les contraintes à son adoption ont été identifiées. Des entretiens semi-directifs ont été conduits dans six villages auprès de 120 ménages dont 72 adoptants de la régénération naturelle assistée et 48 non-adoptants, soit 36 adoptants et 24 non-adoptants dans chacune des deux zones agro-climatiques. Les résultats montrent que les rendements des cultures (mil et arachide), la production fruitière et la production de bois sont plus élevés dans les parcelles des adoptants. Il ressort que dans le centre-nord du bassin arachidier, $3 \%$ des adoptants de cette méthode et $14 \%$ des non-adoptants ne parviennent pas à couvrir leurs besoins alimentaires au-delà de 4 mois après la récolte. Dans le centre sud du bassin arachidier, c'est le cas de $0 \%$ des adoptants et $4 \%$ des non-adoptants. Dans la région centre-sud, un ménage adoptant la méthode gagne 72,65 \$US par saison en moyenne sur la vente de produits forestiers non ligneux, un ménage non-adoptant gagne 29,73 \$US. La régénération naturelle assistée joue donc un rôle important dans l'amélioration des moyens de subsistance des ménages agricoles. Cependant, il a été identifié des contraintes à la diffusion de cette pratique dans la zone d'étude, d'ordre naturel, anthropique et institutionnel.

Abstract. Farmer managed natural regeneration (FMNR) is a practice to help woody cover that is useful to local populations to naturally regenerate. It is practised in Senegal 
as in the whole West Africa. Woody plants provide a large range of goods and services to farm households. This study aims at comparing the socio-economic situation between farm households who practice intensive FMNR and the ones who do not, in two agro-climatic zones of Senegal: the Northern Center and the Southern Center groundnut basin of Senegal (NCGB and SCGB, respectively). In addition, the constraints this practice currently faces were identified. Semi-directing survey was conducted on 120 farm households sampled in six villages, including 72 FMNR adopters and 48 non-adopters, i.e., 36 adopters and 24 non-adopters in each agro-climatic zone. The results show that crop yields (millet and peanut), as well as fruit and wood productions are higher in the plots in which intensive FMNR is practiced. In addition, $3 \%$ of adopters against $14 \%$ of non-adopters in the SCGB, and $0 \%$ of adopters against $4 \%$ of non-adopters in the NCGB do not meet their annual food needs over more than 4 months. Furthermore, households practicing intensive FMNR in the SCGB on average gain 72.65 USD per season from selling non-timber products against 29.73 USD for the others. Consequently, FMNR contributes to improving livelihoods of farm households. However, it has been identified in the studied area natural, anthropic and institutional constraints that the dissemination of the FMNR practice has to cope with.

\section{Introduction}

Limportance des parcs agroforestiers est bien connue en Afrique de l'Ouest. C'est une pratique qui existe depuis des siècles. Ces parcs fournissent du bois, des fruits et des feuilles consommés sur l'exploitation ou commercialisés, pour l'alimentation et la pharmacopée du ménage et comme fourrage. Ils fournissent aussi des services agroécologiques tels que la fertilisation des sols et l'amélioration du microclimat.

Cependant, les parcs agroforestiers sont menacés en raison du vieillissement des arbres. L'absence de leur régénération peut être attribuée à des facteurs environnementaux (changement climatique), socio-économiques (pâturage, incendies et élimination des rejets spontanés du parc) et politiques (code forestier). Pour faire face à cette situation, les agriculteurs investissent dans la protection et la gestion des arbres et des buissons qui se régénèrent naturellement dans leurs champs. Cette pratique s'appelle la régénération naturelle assistée. De nombreuses études menées en Afrique de l'Ouest ont montré les avantages agroécologiques de cette pratique (Badji et al., 2015; Camara et al., 2017) et son rôle socio-économique pour les populations rurales (Butari et al., 2004; Belemvire et al., 2008; Botoni et al., 2010). Cette pratique agroforestière permet notamment de (re)créer un couvert ligneux (Larwanou et Tougiani, 2008; Larwanou et al., 2010; Botoni et al., 2010). Cette pratique séculaire consiste à épargner et à entretenir les espèces ligneuses se régénérant spontanément dans la parcelle cultivée, en les maintenant à des densités suffisamment basses pour éviter la compétition pour les ressources avec la culture associée (Samaké et al., 2011). Elle se distingue de la plupart des autres pratiques agroforestières en ce qu'elle repose entièrement sur le maintien et l'entretien de souches et de plantules d'espèces autochtones déjà en place et ne nécessitant donc aucune pépinière ou plantation.

La pratique de la régénération naturelle assistée a commencé au Niger dans les années 1980 où près de cinq millions d'hectares ont été revégétalisés en utilisant cette méthode (Reij et al., 2009). Au Sénégal, son développement est moins important. Néanmoins, des initiatives ont été prises dans certaines localités : Tivaouane dans la 
région de Thiès (Diallo, 1992), Kaffrine (Bakhoum et al., 2012); Khatre Sy dans la région de Thiès (Badji et al., 2015). Certains avantages de la régénération naturelle assistée ont été étudiés, mais pas quantifiés en termes social et économique (moyens de subsistance). Le but du travail présenté ici est d'évaluer les avantages socio-économiques que les ménages agricoles tirent de cette pratique et d'identifier ses contraintes.

\section{"Méthodologie}

En général, les études d'impact d'une pratique comparent une cohorte d'utilisateurs de la pratique à un groupe témoin idéalement avant et après la mise en place. Cependant, la régénération naturelle assistée, ancienne et généralisée, ne permet pas une telle approche. Dans la zone d'étude, toutes les exploitations contiennent des arbres et des arbustes qui se régénèrent plus ou moins. Pour autant, il est possible de distinguer, d'une part, les exploitations ayant de vieux arbres en faible densité que nous appellerons les «non-adoptants»; d'autre part, celles ayant de jeunes arbres en densité élevée que nous appellerons les «adoptants».

La régénération naturelle assistée concerne un mélange d'arbres et d'arbustes d'âges différents dont seuls les arbres matures fournissent des compléments d'approvisionnement suffisamment abondants et intéressants pour les ménages. Cela implique que ces ménages consacrent du temps et un travail considérable aux jeunes peuplements. Une simple comparaison entre des pratiquants assidus de la régénération naturelle assistée sur des jeunes peuplements non encore productifs et d'autres exploitants apparemment beaucoup moins assidus à cette pratique, car possédant un peuplement déjà suffisamment productif, conduirait à la conclusion erronée que «la régénération naturelle assistée conduit à une baisse des avantages économiques ».

Une approche rigoureuse permettant d'évaluer les impacts de la régénération naturelle assistée est donc de tenir compte du flux des avantages produits pour les adoptants sur un temps suffisamment long. Pour répondre à cet objectif, nous avons prévu dans cette étude de :

- faire une typologie des techniques de régénération naturelle assistée pratiquées pour classer ensuite les ménages dans le groupe des «adoptants» ou des «non-adoptants»;

- établir, sur une durée assez longue, les relations entre les plantes ligneuses concernées et les avantages attendus tels que l'augmentation des rendements des cultures associées et de l'élevage, les compléments de production apportés par les produits forestiers ligneux et non ligneux et leurs valeurs marchandes.

\section{Zone d'étude}

Létude a été menée dans les zones centre-nord (CNBA) et centre-sud (CSBA) du bassin arachidier du Sénégal qui ont bénéficié de l'intervention de projets et d'ONG ayant pour but de promouvoir la régénération naturelle assistée. Elle a concerné la commune de Niathène dans la région de Thiès (pluviométrie de 200 à $500 \mathrm{~mm}$, projet Previnova) et la commune de Diakhac dans la région de Fatick (pluviométrie de 500 à $700 \mathrm{~mm}$, l'ONG World vision) (fig. 11.1). Dans chaque commune, trois 
villages ont été choisis sur la base du degré d'adoption de la régénération naturelle assistée et de manière à regrouper des effectifs de ménages qui la pratiquent à des degrés divers. À Diakhao, le choix a porté sur les villages de Ndoffanemade, Diok et Boffpoupouye. À Niakhène, il s'agit des villages de Niakhène, Khatre Sy et Sam Dieng.

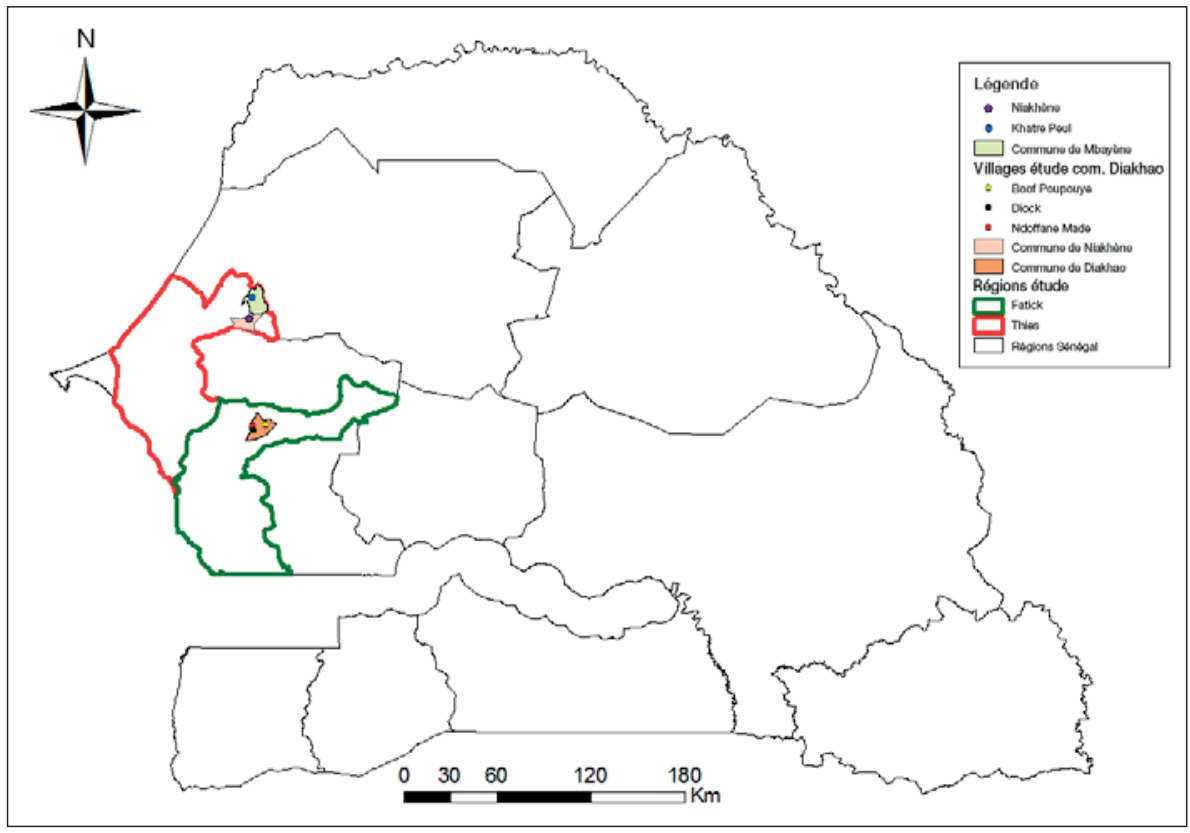

Figure 11.1. Localisation des zones d'étude au Sénégal.

\section{Choix des ménages}

Dans chaque village, l'échantillonnage a porté sur 20 ménages (12 adoptants et 8 nonadoptants), soit un total de 120 ménages pour les six villages. Dans cette étude, la définition des ménages «adoptants » et «non-adoptants» est fondée sur la densité et des diamètres des arbres (DHP - diamètre à hauteur de poitrine). Des sous-groupes des ménages agricoles «adoptants» sont proposés en fonction du nombre d'arbres dans leurs champs. Pour les deux catégories de ménages, la densité de peuplement devait être supérieure ou égale à 100 arbres/ha (tab. 11.1).

Tableau 11.1. Typologie des adoptants et des non-adoptants en fonction de la distribution des peuplements d'arbres dans les champs (diamètre à hauteur de poitrine, DHP et densité).

\section{Densité d'arbres en fonction du diamètre}

\begin{tabular}{lccc}
\hline Diamètre, DHP $(\mathbf{c m})$ & $\mathbf{2 0}$ & $\mathbf{2 0 - 4 0}$ & $>\mathbf{4 0}$ \\
\hline Adoptants & $35-40$ arbres/ha & $40-65$ arbres/ha & $\geq 20$ arbres/ha \\
\hline Non-adoptants & $<10$ arbres/ha & - & $\geq 100$ arbres/ha \\
\hline
\end{tabular}




\section{Méthode de collecte et d'analyse des données}

La méthodologie a consisté à faire la caractérisation socio-économique des ménages. Les productions forestières de l'exploitation (bois de chauffe, bois de service, fourrage, feuilles et fruits) ont été quantifiées seulement au centre-nord du bassin arachidier. Les enquêtes socio-économiques, notamment l'entretien semi-directif auprès des chefs de ménage, ont été privilégiées et réalisées lors de l'inventaire des terres et des parcelles cultivées. Ces enquêtes ont permis de collecter des informations de base sur les ménages, la tenure foncière des parcelles qu'ils cultivent, le droit de propriété et d'utilisation des terres en distinguant les droits à court terme ( 1 à 5 ans) et à long terme (+ de 5 ans), le droit de planter des arbres, d'exploiter les arbres ou de les transmettre aux héritiers. Ces droits sont déterminants dans l'évaluation du degré de liberté des ménages dans la gestion des terres et des ressources qu'elles produisent.

La quantité de bois extraite des parcelles agroforestières a été estimée à partir du nombre de chargements de charrette (environ un stère) de bois que les exploitants ont récoltés. La quantité de produits forestiers non ligneux (fourrage et compléments alimentaires) récoltés a été estimée par le nombre de bassines de $20 \mathrm{~kg}$. Les productions agricoles associées aux essences ligneuses, notamment le mil et l'arachide, ont été quantifiées à l'aide du nombre de sacs de $100 \mathrm{~kg}$ récoltés, ainsi que par les superficies emblavées. Les valeurs marchandes de tous ces produits agroforestiers ont été obtenues auprès des ménages et complétées par des enquêtes effectuées sur les marchés. Les données ont été traitées par des méthodes d'analyse descriptive sur tableur Excel pour Windows. Un t-test a permis la comparaison des moyennes à l'aide du logiciel R (3.4.2) pour Windows.

\section{Mésultats}

\section{Contexte socio-économique de la pratique de la régénération naturelle assistée}

\section{Structure des ménages et caractéristiques sociologiques des chefs de ménage}

Lanalyse des données du tableau 11.2 montre que, quelle que soit la zone agro-climatique, il y a peu de différence du nombre moyen de personnes par ménage, ainsi que leur répartition par classe d'âge entre les ménages adoptants et les non-adoptants.

Lanalyse des données du tableau 11.3 montre que les chefs de ménage sont en très grande majorité (95-100\% des ménages) des hommes, quelle que soit la région agroclimatique et le statut adoptant ou non-adoptant. Les rares femmes chefs de ménage sont essentiellement des veuves.

Dans le centre-nord du bassin arachidier, les chefs de ménage adoptants la régénération naturelle assistée sont majoritairement monogames (69\% des ménages). Les chefs de ménages non-adoptants sont majoritairement polygames (57\% des ménages). Dans le centre-sud du bassin arachidier, les chefs de ménage monogames sont les plus nombreux quel que soit le statut adoptant ou non-adoptant (58-59\% des ménages). 
Les chefs de ménage ayant reçu une éducation sont plus nombreux chez les ménages adoptants que chez les non-adoptants quelle que soit la zone agro-climatique (82\% des ménages au centre-nord et $67 \%$ au centre-sud du bassin arachidier), mais avec une proportion d'analphabètes (rapport nombre d'analphabètes non-adoptants sur le nombre d'analphabètes adoptants) 1,6 fois plus élevée dans le centre-nord du bassin arachidier et 1,3 fois dans le centre-sud.

Tableau 11.2. Nombre moyen de personnes par classe d'âges et genre selon l'adoption de la régénération naturelle assistée dans le centre-nord et dans le centre-sud du bassin arachidier.

\begin{tabular}{|c|c|c|c|c|c|}
\hline \multirow{2}{*}{ 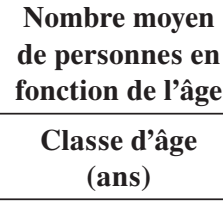 } & \multirow[b]{2}{*}{ Sexe } & \multicolumn{2}{|c|}{$\begin{array}{c}\text { Centre-nord du bassin } \\
\text { arachidier }\end{array}$} & \multicolumn{2}{|c|}{$\begin{array}{l}\text { Centre-sud du bassin } \\
\text { arachidier }\end{array}$} \\
\hline & & $\begin{array}{c}\text { Adoptants } \\
\text { (36) }\end{array}$ & $\begin{array}{c}\text { Non-adoptants } \\
\text { (24) }\end{array}$ & $\begin{array}{c}\text { Adoptants } \\
\text { (36) }\end{array}$ & $\begin{array}{c}\text { Non-adoptants } \\
\text { (24) }\end{array}$ \\
\hline \multirow{2}{*}{$<16$} & Hommes & 3 & 3 & 2 & 2 \\
\hline & Femmes & 3 & 3 & 2 & 2 \\
\hline \multirow{2}{*}{$16-59$} & Hommes & 3 & 2 & 2 & 3 \\
\hline & Femmes & 4 & 3 & 2 & 2 \\
\hline \multirow{2}{*}{$>59$} & Hommes & 1 & 1 & 1 & 1 \\
\hline & Femmes & 1 & 1 & 1 & 1 \\
\hline $\begin{array}{l}\text { Nombre moyen } \mathrm{d} \\
\text { par ménage tous }\end{array}$ & $\begin{array}{l}\text { rsonnes } \\
\text { confondus }\end{array}$ & 15 & 13 & 10 & 11 \\
\hline
\end{tabular}

Tableau 11.3. Comparaison des caractéristiques sociologiques des chefs de ménage adoptants et non-adoptants de la régénération naturelle assistée dans le bassin arachidier du Sénégal.

\begin{tabular}{|c|c|c|c|c|c|}
\hline \multirow{2}{*}{$\begin{array}{c}\text { Chef } \\
\text { de ménage }\end{array}$} & \multirow{2}{*}{$\begin{array}{l}\text { Sexe et niveau } \\
\text { d'éducation }\end{array}$} & \multicolumn{2}{|c|}{$\begin{array}{c}\text { Centre-nord } \\
\text { du bassin arachidier }\end{array}$} & \multicolumn{2}{|c|}{$\begin{array}{c}\text { Centre-sud } \\
\text { du bassin arachidier }\end{array}$} \\
\hline & & $\begin{array}{l}\text { Adoptants } \\
\text { (36) }\end{array}$ & $\begin{array}{c}\text { Non-adoptants } \\
\text { (24) }\end{array}$ & $\begin{array}{l}\text { Adoptants } \\
\text { (36) }\end{array}$ & $\begin{array}{c}\text { Non-adoptants } \\
\text { (24) }\end{array}$ \\
\hline \multirow{2}{*}{ Genre } & Homme $(\%)$ & 97 & 96 & 95 & 100 \\
\hline & Femme $(\%)$ & 3 & 4 & 5 & 0 \\
\hline \multirow{4}{*}{$\begin{array}{l}\text { Situation } \\
\text { matrimoniale }\end{array}$} & Polygame $(\%)$ & 29 & 57 & 29 & 36 \\
\hline & Monogame (\%) & 69 & 35 & 58 & 59 \\
\hline & Célibataire (\%) & 0 & 0 & 8 & 5 \\
\hline & Veuf ou Veuve $(\%)$ & 3 & 9 & 5 & 0 \\
\hline \multirow{3}{*}{ Éducation } & $\begin{array}{c}\text { Formelle }(\%) \\
\text { (primaire, secondaire, } \\
\text { supérieure) }\end{array}$ & 63 & 48 & 46 & 29 \\
\hline & $\begin{array}{c}\text { Informelle } \\
(\%) \text { (coranique, } \\
\text { alphabétisation) }\end{array}$ & 20 & 23 & 20 & 27 \\
\hline & Analphabète (\%) & 18 & 29 & 33 & 44 \\
\hline
\end{tabular}




\section{Gouvernance des ressources}

Lobservation des données du tableau 11.4 révèle que l'essentiel des superficies exploitées se trouve dans les champs de brousse, c'est-à-dire les plus éloignés des habitations du village. Au centre-sud du bassin arachidier, les ménages nonadoptants disposent, par rapport aux non-adoptants, de champs à des distances intermédiaires entre les champs de case et les champs de brousse ( «plein champs» tab. 11.4) et d'une superficie cultivée moyenne un peu plus élevée ( 5,5 ha) que celle des adoptants (5,1 ha). À l'inverse, au centre-nord du bassin arachidier, la superficie cultivée moyenne est un peu plus élevée chez les adoptants (11,8 ha) que chez les non-adoptants (10,6 ha). Le mode d'acquisition des champs le plus courant est l'héritage. La mise en valeur de ces superficies est basée à plus de $80 \%$ sur le droit d'usage à long terme, ce qui procure aux chefs de ménage la possibilité de planter et d'exploiter les arbres, de faire des aménagements et de transmettre les champs à leurs héritiers.

Tableau 11.4. Comparaison de la gouvernance des ressources entre adoptants et nonadoptants de la RNA dans le bassin arachidier du Sénégal.

\begin{tabular}{|c|c|c|c|c|c|}
\hline \multirow{2}{*}{\multicolumn{2}{|c|}{$\begin{array}{l}\text { Modalités de gouvernance } \\
\text { des ressources }\end{array}$}} & \multicolumn{2}{|c|}{$\begin{array}{c}\text { Centre-nord du bassin } \\
\text { arachidier }\end{array}$} & \multicolumn{2}{|c|}{$\begin{array}{l}\text { Centre-sud du bassin } \\
\text { arachidier }\end{array}$} \\
\hline & & $\begin{array}{l}\text { Adoptants } \\
\text { (36) }\end{array}$ & $\begin{array}{c}\text { Non-adoptants } \\
\text { (24) }\end{array}$ & $\begin{array}{l}\text { Adoptants } \\
\text { (36) }\end{array}$ & $\begin{array}{c}\text { Non-adoptants } \\
\text { (24) }\end{array}$ \\
\hline \multirow{3}{*}{$\begin{array}{l}\text { Types } \\
\text { de champs }\end{array}$} & $\begin{array}{l}\text { Champs de case } \\
\text { (à proximité } \\
\text { du village) }(\%)\end{array}$ & 11 & 21 & 44 & 46 \\
\hline & $\begin{array}{l}\text { Champs de brousse } \\
\text { (éloignés du } \\
\text { village) }(\%)\end{array}$ & 89 & 79 & 56 & 50 \\
\hline & $\begin{array}{l}\text { Plein champs } \\
\text { (à distance } \\
\text { intermédiaire entre } \\
\text { les deux types de } \\
\text { champ précédents) } \\
(\%)\end{array}$ & 0 & 0 & 0 & 4 \\
\hline \multirow{3}{*}{$\begin{array}{l}\text { Mode } \\
\text { d'acquisition } \\
\text { des terres }\end{array}$} & Héritage $(\%)$ & 89 & 67 & 89 & 92 \\
\hline & Emprunt (\%) & 8 & 25 & 11 & 4 \\
\hline & Don $(\%)$ & 3 & 8 & 0 & 4 \\
\hline \multirow{5}{*}{$\begin{array}{l}\text { Droits } \\
\text { d'usages } \\
\text { des terres }\end{array}$} & À court terme $(\%)$ & 11 & 13 & 6 & 4 \\
\hline & À long terme (\%) & 89 & 88 & 94 & 96 \\
\hline & $\begin{array}{l}\text { Plantation } \\
\text { et exploitation } \\
\text { d'arbres }(\%)\end{array}$ & 85 & 88 & 97 & 99 \\
\hline & $\begin{array}{l}\text { Aménagement } \\
\text { (ex anti érosif) (\%) }\end{array}$ & 86 & 88 & 97 & 99 \\
\hline & $\begin{array}{l}\text { Transmission par } \\
\text { héritage }(\%)\end{array}$ & 75 & 75 & 92 & 99 \\
\hline
\end{tabular}




\section{Dotation en biens d'équipement selon l'adoption}

Lanalyse des données concernant les biens d'équipement (tab. 11.5) révèle que dans les deux régions étudiées, la majorité des ménages adoptants possède au moins une maison en dur (64-83\%); tous les ménages ayant une maison (en banco, en dur ou les deux). Une grande proportion de ménages adoptants dispose de moyens de travail et de déplacement (cheval et charrette), cette proportion est moindre chez les ménages non-adoptants. La possession du téléphone et d'un téléviseur ne semble pas dépendre du statut d'adoptant ou de non-adoptant.

Tableau 11.5. Comparaison des équipements entre adoptants et non-adoptants de la régénération naturelle assistée dans le bassin arachidier du Sénégal.

\begin{tabular}{lcccc}
\hline \multicolumn{1}{c}{ Équipements } & \multicolumn{2}{c}{ Centre-nord du bassin arachidier } & \multicolumn{2}{c}{ Centre-sud du bassin arachidier } \\
\cline { 2 - 5 } & $\begin{array}{c}\text { Adoptants } \\
\mathbf{( 3 6 )}\end{array}$ & $\begin{array}{c}\text { Non-adoptants } \\
\mathbf{( 2 4 )}\end{array}$ & $\begin{array}{c}\text { Adoptants } \\
\mathbf{( 3 6 )}\end{array}$ & $\begin{array}{c}\text { Non-adoptants } \\
(\mathbf{2 4 )}\end{array}$ \\
\hline Maison en dur (\%) & 83 & 54 & 64 & 38 \\
\hline Maison en banco (\%) & 31 & 63 & 64 & 71 \\
\hline Téléviseur (\%) & 31 & 25 & 22 & 46 \\
\hline Téléphone (\%) & 22 & 37 & 81 & 83 \\
\hline Mobylette (\%) & 0 & 0 & 13,8 & 0 \\
\hline Charrette (\%) & 92 & 83 & 56 & 50 \\
\hline Cheval (\%) & 100 & 67 & 78 & 50 \\
\hline Âne $(\%)$ & 56 & 58 & 78 & 46 \\
\hline
\end{tabular}

\section{Effets de la régénération naturelle assistée sur les productions agroforestières}

\section{Sur les productions forestières}

Dans le centre-nord du bassin arachidier, les adoptants de la régénération naturelle assistée ont une production fruitière plus élevée que celle des non-adoptants ( $\mathrm{p}=0,002)$, alors que c'est l'inverse $(\mathrm{p}<0,0001)$ dans le centre-sud du bassin arachidier (fig. 11.2). Au centre-nord du bassin arachidier, seule région échantillonnée pour quantifier les productions forestières, la quantité de bois récolté dans les champs des adoptants de la régénération naturelle assistée est supérieure $(\mathrm{p}<0,0001$, tab. 11.6) à celle des non-adoptants, mais les productions de fourrage et de feuilles destinées à la consommation du ménage sont significativement inférieures $(p=0,001 ; p=0,043)$.

\section{Sur les productions agricoles}

\section{et les quantités de fertilisants apportés}

Aussi bien dans le centre-nord que le centre-sud du bassin arachidier, la figure 11.3 montre que la production des deux principales spéculations de l'agriculture pluviale, le mil et l'arachide, est plus importante chez les ménages adoptants que chez les 
non-adoptants : $\mathrm{p}<0,0001$ au centre-nord du bassin arachidier et $\mathrm{p}=0,003 \mathrm{au}$ centre-sud du bassin arachidier pour le mil; $\mathrm{p}<0,0001$ au centre-nord du bassin arachidier et $\mathrm{p}<0,0001$ au centre-sud du bassin arachidier pour l'arachide. Il ressort également que pour les ménages du centre-nord du bassin arachidier (fig. 11.4), les adoptants apportent beaucoup plus de fumier ( $p<0,0001)$ et beaucoup moins d'engrais minéral $(\mathrm{p}<0,0001)$ que les non-adoptants. Les ménages apportent très peu d'engrais minéral $(\mathrm{p}=0,06)$ dans le centre-sud du bassin arachidier, quel que soit leur statut adoptant ou non-adoptant, mais ce sont les non-adoptants qui apportent le plus de fumier $(\mathrm{p}<0,0001)$.

Tableau 11.6. Comparaison des quantités de produits forestiers entre adoptants et nonadoptants de la régérnération naturelle assistée dans le centre-nord du bassin arachidier du Sénégal.

\begin{tabular}{lccc}
\hline \multicolumn{1}{c}{ Produits forestiers } & Adoptants & Non-adoptants & p-value \\
\hline Production de bois (nombre de charrettes) & $6 \pm 0,3$ & $4 \pm 0,2$ & $\mathrm{p}<0,0001$ \\
\hline Fourrage (nombre de sacs de $100 \mathrm{~kg}$ ) & $12 \pm 0,9$ & $16 \pm 0,7$ & $\mathrm{p}<0,0001$ \\
\hline $\begin{array}{l}\text { Feuilles destinées à la consommation } \\
\text { du ménage (nombre de bassine de } 20 \mathrm{~kg} \text { ) }\end{array}$ & $28 \pm 1,3$ & $32 \pm 1,5$ & $\mathrm{p}=0,046$ \\
\hline
\end{tabular}

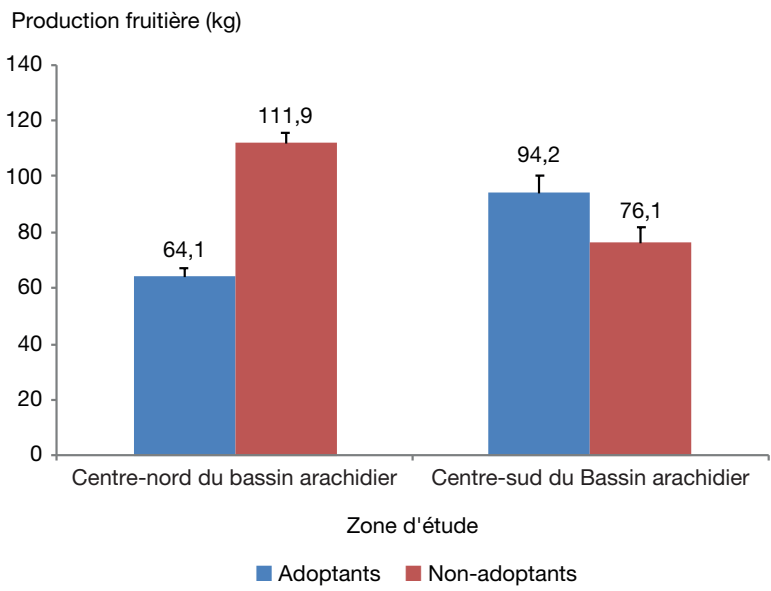

Figure 11.2. Comparaison de la production fruitière des adoptants et non-adoptants de la régénération naturelle assistée dans le bassin arachidier du Sénégal.

\section{Effets de la régénération naturelle assistée sur l'économie des ménages et la sécurité alimentaire}

Les résultats montrent que dans le centre-nord et le centre-sud du bassin arachidier, la proportion des ménages qui pratiquent le commerce de fruits forestiers est plus importante chez les ménages adoptants (fig. 11.5). Quelle que soit la zone, un ménage adoptant la régénération naturelle assistée gagne en moyenne 72,65 \$US par saison sur la vente de produits forestiers non ligneux contre 29,73 \$US pour un ménage non-adoptant. 


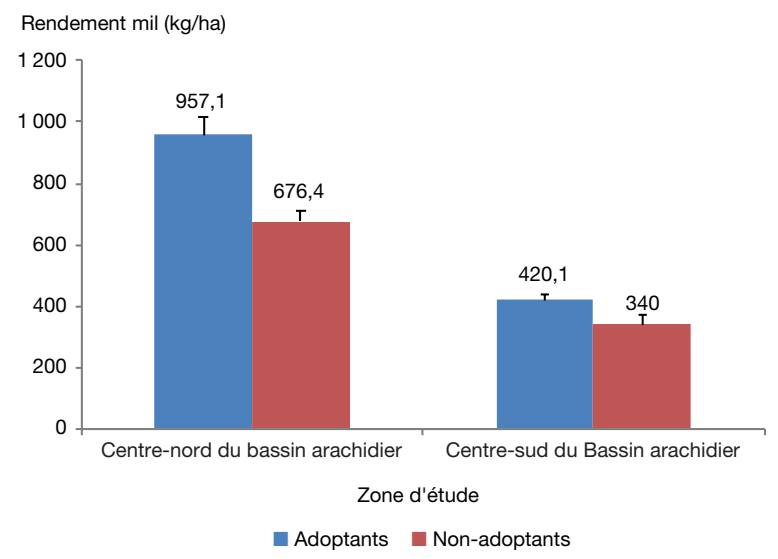

Figure 11.3a. Comparaison de la production de mil entre adoptants et non-adoptants de la régénération naturelle assistée dans le bassin arachidier du Sénégal.

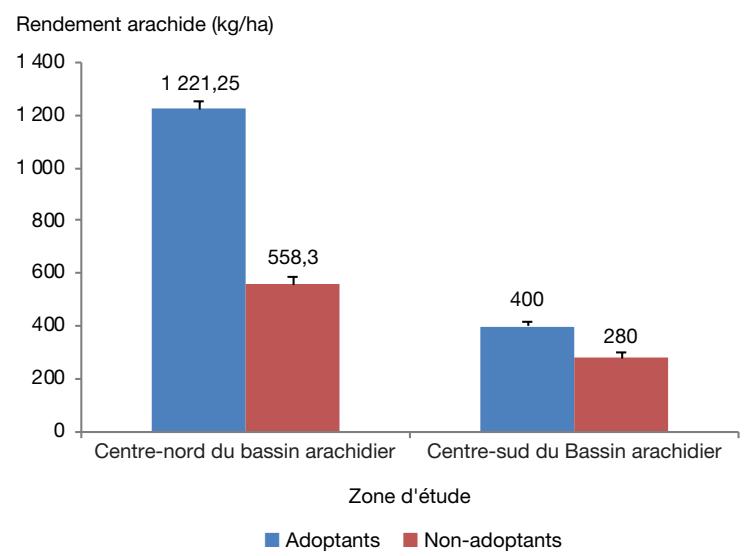

Figure 11.3b. Comparaison de la production d'arachide entre adoptants et non-adoptants de la régénération naturelle assistée dans le bassin arachidier du Sénégal.

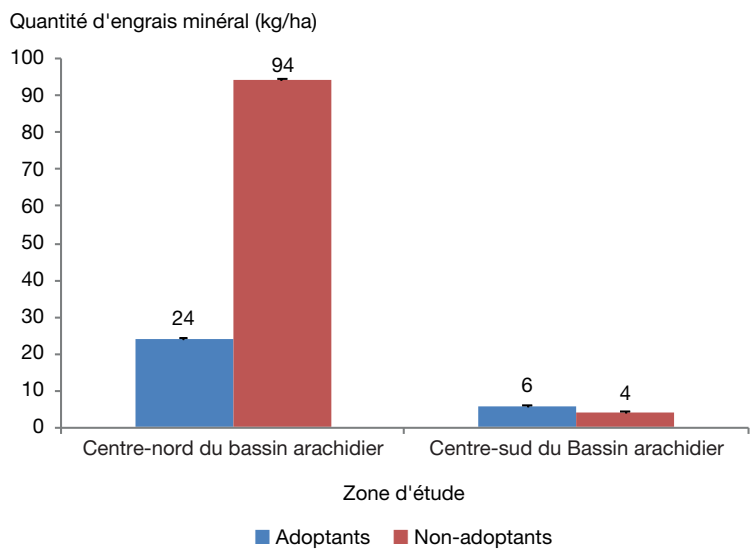

Figure 11.4a. Quantité d'engrais minéral utilisée entre adoptants et non-adoptants de la régénération naturelle assistée dans le bassin arachidier du Sénégal. 


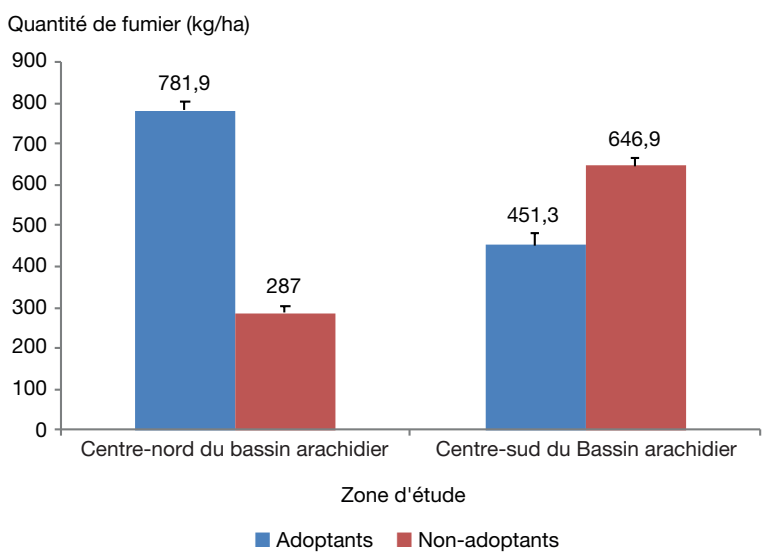

Figure 11.4b. Quantité de fumier utilisée entre adoptants et non-adoptants de la régénération naturelle assistée dans le bassin arachidier du Sénégal.

Si l'on considère la durée de la consommation de la production agricole annuelle, il ressort que dans la région centre-sud, $14 \%$ des ménages non-adoptants de la régénération naturelle assistée ne parviennent pas à couvrir leurs besoins alimentaires au-delà de 4 mois, contre seulement $3 \%$ des ménages adoptants; dans le centre-nord, cela ne concerne que $4 \%$ des ménages non-adoptants (fig. 11.6). Dans la région centre-nord du bassin arachidier, quel que soit leur statut, la majorité des ménages (61-67\%) vit grâce à ses récoltes au-delà d'un an, tandis que dans la région centre-sud, 75 à $80 \%$ des ménages ne disposent pas de plus de 11 mois d'autonomie alimentaire (fig. 11.6). Leffet de la régénération naturelle assistée sur la sécurité alimentaire des ménages dépend donc fortement de la zone agro-climatique.

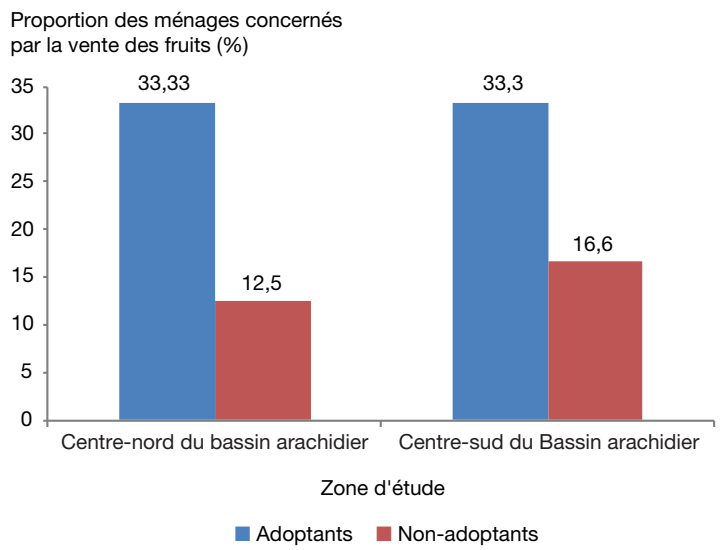

Figure 11.5. Comparaison de la proportion des ménages adoptants et non-adoptants concernés par la vente des fruits forestiers selon le niveau d'adoption de la régénération naturelle assistée dans le bassin arachidier du Sénégal. 


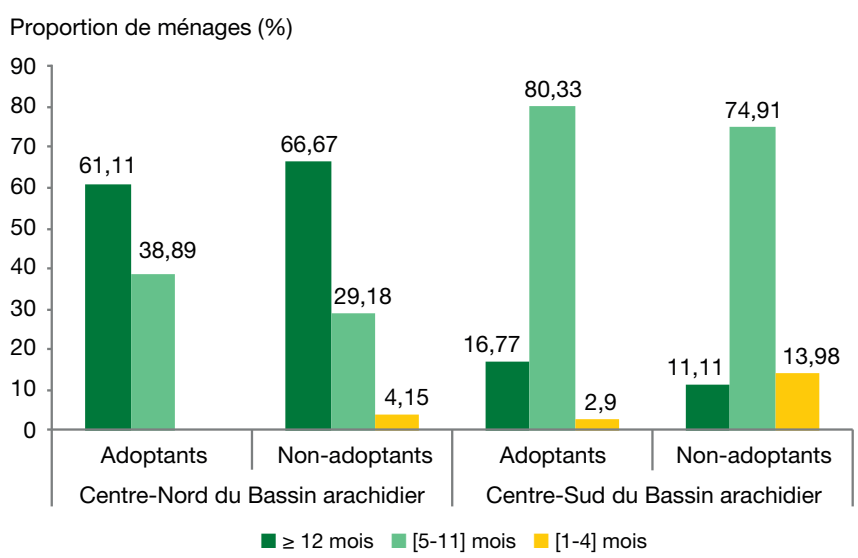

Figure 11.6. Comparaison de la durée de consommation de la production agricole entre adoptants et non-adoptants de la régénération naturelle assistée dans le bassin arachidier du Sénégal.

\section{Les contraintes à la diffusion et à l'extension de la régénération naturelle assistée}

Selon les chefs de ménage adoptants et non-adoptants de la régénération naturelle assistée, les contraintes à la diffusion de cette pratique sont naturelles, anthropiques et institutionnelles.

Les contraintes naturelles. Pour $5 \%$ des chefs de ménage adoptants et $15 \%$ des non-adoptants, la survie des arbres issus de la régénération naturelle assistée est tributaire du régime pluviométrique. En effet, la pluviométrie subit de fortes variations spatiales et interannuelles sous ce climat tropical sec; la baisse des précipitations ayant pour conséquence l'augmentation de la salinité du sol. Pour $2 \%$ des chefs de ménage adoptants et $6 \%$ des non-adoptants, les rejets spontanés disponibles sont insuffisants. La croissance des essences forestières est trop lente. C'est le cas de Faidherbia albida, l'une des principales espèces conservées dans les espaces agraires du bassin arachidier.

Les contraintes anthropiques. Selon $46 \%$ des chefs de ménage adoptants et $48 \%$ des non-adoptants, la coupe illicite des arbres effectuée par les éleveurs transhumants pour alimenter leur bétail constitue la contrainte la plus importante. Pour $20 \%$ des chefs de ménage adoptants et $15 \%$ des non-adoptants, la satisfaction des besoins en bois de feu et de service constitue également une contrainte parce qu'elle occasionne des coupes frauduleuses de jeunes arbres dans les champs. Enfin, des pratiques agricoles destructrices (défrichement, dessouchage et brûlage) sont également identifiées comme des freins à la diffusion de la régénération naturelle assistée par $12 \%$ des chefs de ménage adoptants et $3 \%$ des non-adoptants.

Les contraintes institutionnelles. Pour $12 \%$ des chefs de ménage adoptants et nonadoptants, l'absence de conventions locales relatives à la divagation des animaux est la principale contrainte. Le code forestier ne constitue pas une contrainte, car toutes les terres sont héritées et confèrent aux propriétaires tous les droits d'usages (plantation, investissement, transmission, etc.). 


\section{" Discussion}

Dans la zone d'étude, le fait que les chefs de ménage responsables de la gestion des ressources naturelles soient essentiellement des hommes compromet le rôle que la femme pourrait avoir dans l'adoption de nouvelles technologies agroforestières, malgré leur intérêt majeur en raison de leur rôle nourricier au sein des ménages. Sanogo et al. (2004) et Lafleur (2008) ont montré que les femmes disposent de très peu de pouvoir de décision, elles sont souvent tenues à l'écart de la gestion du foncier rural et des ressources naturelles. Sene (1994) et Wedum et al. (1996) indiquent que les femmes n'étant pas propriétaires de terre, elles ne peuvent pas décider de leur mode de gestion. Dans la zone d'étude, le mode d'acquisition de la propriété le plus courant pour une femme est l'héritage par veuvage. Il lui confère alors tous les droits d'utilisation des terres et lui permet d'adopter des technologies innovantes, telle la régénération naturelle assistée.

Nos résultats confirment que l'adoption de la régénération naturelle assistée est liée au niveau d'étude des chefs de ménage. Marou et al. (2002) avaient déjà indiqué que l'éducation des ménages est un facteur déterminant favorisant l'adoption de cette pratique. Wu et Babcock (1998) ont également montré que les agriculteurs instruits sont plus aptes à adopter une nouvelle technologie.

Au départ de l'étude, les ménages adoptants disposaient déja de plus de moyens que les ménages non-adoptants (maisons en dur, chevaux). Avec plus de moyens de locomotion (mobylette, cheval) que les autres, ils peuvent mieux surveiller leurs champs et, par conséquent, adoptent davantage la régénération naturelle assistée. Gapihan (1998) et Sanogo et al. (2004) ont montré que «la richesse» de l'agriculteur avait une influence certaine sur la décision d'adopter une technologie ou une pratique agroforestière innovante. Louppe et Yossi (2000) confirment enfin que les technologies proposées par la recherche et le développement ne sont adoptées que si les paysans ont les moyens nécessaires ou y trouvent un intérêt économique. Ladoption de la régénération naturelle assistée joue donc un rôle sur l'amélioration des conditions de vie des ménages les plus aisés.

Le rendement des cultures de mil et d'arachide, plus élevés dans les parcelles des adoptants que dans celles des non-adoptants, est vraisemblablement dû à l'effet d'«îlots de fertilité» lié à la présence des arbres. C'est pourquoi plusieurs auteurs ont indiqué que la pratique de la régénération naturelle assistée améliore la fertilité des sols (Larwanou et al., 2006; Botoni et Reij, 2009; Larwanou et al., 2010; Rinaudo et al., 2010). En particulier, les arbres de la famille des légumineuses qui fournissent de l'azote au sol, comme Faidherbia albida (Peltier, 1996), dominent dans les deux zones agro-climatiques étudiées ici. Dans le centre-sud du bassin arachidier, en augmentant le rendement dans les parcelles agricoles, la régénération naturelle assistée permet aux ménages qui l'adoptent un niveau de sécurité alimentaire plus élevé que celui des non-adoptants. Dans le centrenord du bassin arachidier, notre étude n'a pas mis en évidence un effet aussi net de la régénération naturelle assistée sur la sécurité alimentaire. Les raisons restent à analyser. En revanche, quelle que soit la région, les revenus issus de la vente des produits forestiers, aussi bien ligneux que non ligneux, sont plus élevés chez les ménages adoptants. 
La principale contrainte à la diffusion de la pratique de la régénération naturelle assistée que notre étude a permis d'identifier est la coupe illicite des arbres - par les éleveurs transhumants pour le fourrage ou par les populations pour le bois de chauffe et de service - et la divagation des animaux. Ces résultats confirment ceux obtenus par Sanogo et al., 2004 qui montrent que la divagation animale est une contrainte majeure à l'adoption de nouvelles technologies agroforestières. Larwanou et al. (2010) et Larwanou et Saadou (2012) indiquent aussi que les coupes frauduleuses constituent un facteur important de dégradation. Elles menacent la régénération d'espèces de grande valeur économique.

\section{W Conclusion}

Cette étude a montré que l'adoption de la régénération naturelle assistée joue un rôle important dans l'amélioration des conditions de vie des populations rurales en général, des agriculteurs qui ont adopté cette pratique en particulier, à travers l'augmentation des rendements des cultures et des revenus des ménages. Les adoptants bénéficient d'autant plus des avantages tirés de cette pratique que l'agriculture, l'élevage et le commerce des produits agricoles et forestiers sont les activités prédominantes.

\section{" Remerciements}

Nous remercions le Centre mondial de l'agroforesterie (Icraf), bureau régional de l'Afrique de l'Ouest et du centre-nord du Sahel, notamment Dr. Jules Bayala et Dr. Antoine Kalinganire pour leur collaboration scientifique. Nous sommes redevables aux ménages des communautés rurales du bassin arachidier du Sénégal dont l'expérience, la connaissance, la persévérance et l'amitié ont servi de base à notre compréhension et à nos interprétations.

\section{" Bibliographie}

Badji M., Sanogo D., Coly L., Diatta Y., Akpo L.E., 2015. La régénération naturelle assistée (RNA) comme un moyen de reverdir le bassin arachidier au Sénégal : cas du terroir de Khatre Sy. International journal of biological and chemical sciences, 9(1): 234-245.

Bakhoum C., Ndour B., Akpo L., 2012. Natural regeneration of woody stands in the groundnut basin lands in the sudano-sahelian zone (region of Kaffrine, Senegal). Journal of applied environmental and biological, 2(7): 271-280.

Belemvire A., Maiga A., Sawadogo H., Savadogo M., Ouedraogo S., 2008. Évaluation des impacts biophysiques et socio-économiques des investissements dans les actions de gestion des ressources naturelles au nord du plateau central du Burkina Faso. Rapport de synthèse, version provisoire. http://portails.cilss.bf/IMG/pdf/etudesahelrapportBF.pdf (consulté le 08/04/2019).

Botoni E., Larwanou M., Reij C., 2010. La régénération naturelle assistée (RNA) : une opportunité pour reverdir le Sahel et réduire la vulnérabilité des populations rurales. Le projet majeur africain de la Grande muraille verte, concepts et mise en œuvre, 151-162. Paris : IRD Éditions. https://books.openedition.org/irdeditions/2122?lang=fr (consulté le 08/04/2019). 
Botoni E., Et Reij C., 2009. La transformation silencieuse de l'environnement et des systèmes de production au Sahel : impacts des investissements publics et privés dans la gestion des ressources naturelles. Comité permanent interÉtats de lutte contre la sécheresse dans le Sahel (Cilss), 61 p. http://www.agrhymet.ne/portailCC/images/pdf/Rapport\%20Synthse_Etude_Sahel\%20Final.pdf (consulté le 08/04/2019).

Butari I., Zounhi J.S., Diallo A., 2004. Leçons tirées des expériences de lutte contre la désertification au Sahel. Actes des travaux de l'atelier sous-régional d'échange et de réflexion organisé par le Centre de recherche pour le développement international (Croi), Saly Portudal, Sénégal. Dakar, Croi, $187 \mathrm{p}$.

Camara B.A., Drame M., Sanogo D., Ngom D., Badji M., Diop M., 2017. La régénération naturelle assistée : perceptions paysannes et effets agroécologiques sur le rendement du mil (Pennisetum glaucum L. R.Br.) dans le bassin arachidier au Sénégal. Journal of applied biosciences, 112 : $11025-11034$.

Diallo D., 1992. Bibliographie annotée sur l'utilisation des plantes sauvages, en particulier de la littérature sur des plantes médicinales de l'Afrique occidentale. $36 \mathrm{p}$.

Gapihan L.Y., 1998. Pratiques paysannes d'agroforesterie et capacités d'adaptation au changement. Étude de cas du village de Dessimbblé, département de Korhogo, Côte-d’Ivoire. Mémoire de fin d'études, Master européen Natura. Montpelier : Cnearc, 77 p. + annexe.

Lafleur M., 2008. Recherches et documentation des meilleures pratiques pour la gestion durable des parcs à karité en Afrique de l'Ouest. Programme de Renforcement des capacités des productrices de beurre de karité en Afrique de l'Ouest. Montréal : CECI, 110 p. http://www.laboress-afrique. org/ressources/assets/docP/Document_N0920.pdf (consulté le 08/04/2019).

Larwanou M., Et Saadou M., 2012. Impacts des activités de restauration des terres sur la végétation au Niger. Journal des sciences de l'environnement, 1(1) : 1-15.

Larwanou M., Oumarou L., Snook L., Danguimbo I., 2010. Pratiques sylvicoles et culturales dans les parcs agroforestiers suivant un gradient pluviométrique nord-sud dans la région de Maradi au Niger. Tropicultura, 28(2) : 115-122.

Larwanou M., Tougiani A., 2008. Les techniques de régénération naturelle assistée au Sahel; manuel de formation à l'intention des agents de vulgarisation et des producteurs sahéliens. Icraf/Sahel, $18 \mathrm{p}$.

Larwanou M., Abdoulaye M., Et Reij C., 2006. Étude de la régénération naturelle assistée dans la région de Zinder (Niger). Nouakchott : Usaid, Égat, 56 p.

Louppe D., Yossi H., 2000. Les haies vives en Afrique de l'Ouest sèche et subhumide (bilan des connaissances). In : La jachère en Afrique tropicale rôles, aménagements, alternatives. Floret C. et Pontanier R. (eds), Vol 1. Actes du séminaire international, Dakar, 13-16 avril 1999. John Libbey-Eurotext.

Marou Z.A., Abasse A.T., Bokar M., Niang A., Cheick O.T., 2002. Analyse de l'adoption de la régénération naturelle assistée dans la région de Maradi au Niger. Bamako, Mali. 7 p. https://www. formad-environnement.org/RNA-Maradi_Marou-Zarafi_2002.pdf (consulté le 08/04/2019).

Reij C., Tappan G., Et Smale M., 2009. Agricultural transformation in the Sahel: another kind of «Green revolution ». Ifpri discussion paper $\mathrm{n}^{\circ}$ 914. www.ifpri.org/millionsfeed.

Rinaudo T., 2010. Une brève histoire de la régénération naturelle assistée l'expérience du Niger. Note technique, 27 p. http://www.adaa-ase.com/documents/regeneration-naturelle-assistee.pdf (consulté le 08/04/2019).

Samaké O., Dakouo J.M., Kalinganire A., Bayala J., Koné B., 2011. Régénération naturelle assistée : gestion des arbres champêtres au Sahel. Icraf technical manual, 16. Nairobi, Kenya: World agroforestry centre.

Sanogo D., Ayuck E., Dia Y.K., 2004. Appropriation de technologies agroforestières : cas de la haie vive dans le sud bassin arachidier du Sénégal. La haie vive dans le Sud du bassin arachidier du Sénégal. Études et recherches sahéliennes, 10 : 7-17, Institut du Sahel/Insah.

Sene A., 1994. Étude socio-économique des systèmes à parc dans le bassin arachidier : cas de Sterculia setigera et Cordyla pinnata. Mémoire de confirmation Isra, 94 p. http://intranet.isra.sn/greenstone/collect/tmisra/index/assoc/HASH21c8.dir/doc.pdf (consulté le 08/04/2019). 
Wedum J., Doumbia Y., Sanogho B., Dicko G., Et Cisse O., 1996. Réhabilitation de terres dégradées : le zai, dans le cercle de Djenné (au Mali). In : Techniques traditionnelles de conservation de l'eau et des sols en Afrique. Paris : Éditions Kathala, CDCS et CTA. 95-102.

Wu J., Babcock B.A., 1998. The choice of tillage, rotation, and soil testing practices: Economic and environmental implications. American journal of agricultural economics, 80(3): 494-511. 
Chapitre 12

\title{
Les cultures vivrières associées aux arbustes natifs : un modèle adapté au climat sahélien
}

\author{
Chapuis-Lardy L., Badiane Ndour N.Y., Assigbetse K., Diédhiou I., \\ Balaya R., Cournac L., Founoune-Mboup H., Mc Spadden Gardener B., \\ Ghezzehei T., Jourdan C., Bright M.B., Bogie N., Debenport S., \\ Delay C., Diakhaté S., Sambou D.M. et Dick R.P.
}

\begin{abstract}
Résumé. Basé sur des cas d'étude au Sénégal, ce chapitre dresse le bilan de 15 années de recherches sur le fonctionnement des sols d'agrosystèmes associant plantes cultivées (milarachide) et arbustes natifs au Sahel. Les travaux, publiés pour la plupart, ont montré que Guiera senegalensis et Piliostigma reticulatum redistribuent l'eau dans le sol, stimulent les microorganismes du sol et génèrent des îlots de fertilité au bénéfice de la culture associée. Ainsi, les rendements des cultures sont augmentés, y compris en absence de fertilisants : jusqu'à $+137 \%$ pour le mil en présence de $P$. reticulatum à Nioro-du-Rip. Cela justifie l'intérêt de maintenir ces arbustes dans les champs en appliquant une gestion agroécologique.

Abstract. Based on Senegalese case-studies, this chapter synthetize 15-years of research on the soil functioning of native shurb intercropping systems in the Sahel. Scientifically validated results showed that Guiera senegalensis and Piliostigma reticulatum perform water redistribution within soil profile, affect soil microbial communities and their activities and act as resource islands. Intercropping with woody shrubs resulted in a significant increase in crop yield: up to $+137 \%$ yields for millet associated with $P$. reticulatum at Nioro-du-Rip. The beneficial effects for the associated crops raise interest for developing shrub-based agro-ecological systems.
\end{abstract}




\section{Introduction}

Dans le Sahel, les pratiques agricoles traditionnelles intègrent le risque climatique propre aux zones arides et semi-arides : pluviométrie limitée et concentrée sur quelques mois. Toutefois, l'intensification écologique propose aux agriculteurs des voies d'adaptation à la variabilité interannuelle plus prononcée des précipitations, aux événements extrêmes de plus en plus fréquents (Seneviratne et al., 2012) ou aux évolutions actuelles des sociétés (Masse et al., 2013).

Les arbres et arbustes font partie intégrante du paysage agraire subsaharien. Aux côtés des traditionnels parcs arborés, Guiera senegalensis J.F. Gmel (Combretaceae) et Piliostigma reticulatum D.C. Hochst (Caesalpinioideae), deux arbustes natifs, sont souvent maintenus dans les champs pour de nombreux usages : bois de chauffe, médecine traditionnelle et artisanat (Yelemou et al., 2007; Hernandez et al., 2015). Les paysans d'Afrique subsaharienne commencent à préparer leurs champs pour les cultures annuelles avant les premières pluies; les arbustes sont alors coupés au ras-du-sol pour faciliter le semis. Traditionnellement dans le bassin arachidier du Sénégal, les tiges de diamètre supérieur au centimètre sont récoltées pour être utilisées comme bois de chauffe. Le reste des résidus de coupe est brûlé sur place, fournissant ainsi au sol des éléments minéraux. Une alternative au brûlis consiste à utiliser les résidus du recépage pour constituer un paillis à la surface du sol (mulch), comme cela est parfois pratiqué dans certaines régions du Niger et du Burkina Faso. Larbuste repousse ensuite au cours de la saison lorsque la culture est installée (Lahmar et al., 2012; fig. 12.1).
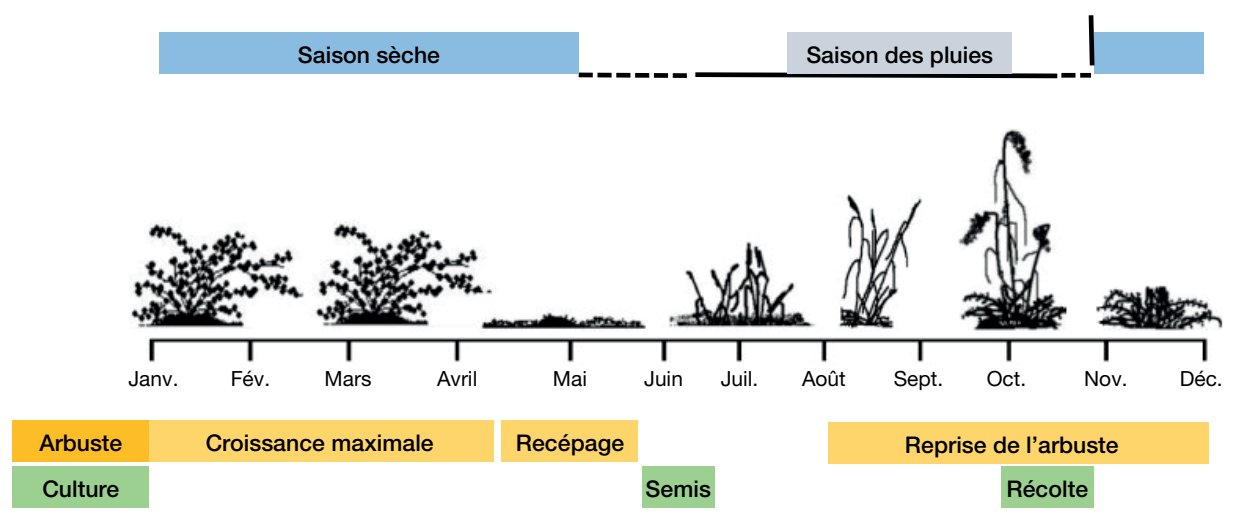

Figure 12.1. Gestion de l'arbuste par recépage et paillis dans les systèmes agroforestiers associant arbustes locaux et céréales en Afrique de l'Ouest (Lahmar et al., 2012).

Bien que l'on retrouve $G$. senegalensis et $P$. reticulatum dans les paysages soudanosahéliens et soudaniens de la côte atlantique (Mauritanie et Sénégal) jusqu'à la mer Rouge (Soudan et Érythrée), ce chapitre se focalise sur les résultats des travaux portant sur ce mode de gestion agroécologique (recépage et paillis) de ces deux arbustes au Sénégal. Ces travaux s’inscrivent dans une dynamique de quinze années de recherches menées en partenariat par des équipes sénégalaises, américaines et françaises, avec le soutien de la National Science Fondation. 


\section{Des rendements améliorés}

\section{Dispositifs expérimentaux}

Deux sites d'étude où prédomine l'un des deux arbustes dans le paysage ont été choisis :

- Guiera senegalensis (Gs) à Keur Matar dans le centre-ouest du Sénégal, près de Thiès;

- Piliostigma reticulatum (Pr) à Nioro-du-Rip dans le sud du bassin arachidier en zone plus humide.

À Keur Matar, le sol, très sableux et lessivé, est classé dans le type arenosol par la FAO (2006) et considéré comme un sol «dior» dans la classification locale (Badiane et al., 2000). À Nioro-du-Rip, le sol est de type lixisol, localement appelé deck-Dior.

Sur ces deux sites, l'Institut sénégalais de recherches agricoles (Isra) a mis en place des essais expérimentaux en 2003.

Dans ces essais, la densité des arbustes a été augmentée et homogénéisée : 1200-1500 touffes arbustives/ha au lieu de quelques centaines en milieu paysan. Dans ces dispositifs, l'arbuste est associé, les années impaires, à une culture de mil (Penisetum glaucum) en rotation, les années paires, avec l'arachide (Arachis hypogea); les résidus de coupe sont restitués au sol.

Ces systèmes d'association culture-arbuste sont comparés à une culture pure, sans arbuste ni mulch. Ces dispositifs sont en blocs aléatoires complets et parcelles divisées (split plot).

Ils comprennent également des apports différenciés d'engrais commerciaux : $0 ; 0,5$; 1; 1,5 fois les doses NPK recommandée slocalement. Les apports recommandés sont :

- pour l'arachide, apport après germination de $9 \mathrm{~kg} \mathrm{~N}, 30 \mathrm{~kg} P$ et $15 \mathrm{~kg} \mathrm{~K} / \mathrm{ha}$ de NPK 6-20-10);

- pour le mil, apport au semis de $22,5 \mathrm{~kg} \mathrm{~N}, 15 \mathrm{~kg}$ P et $15 \mathrm{~kg} \mathrm{~K} / \mathrm{ha}$ de NPK $15-15-15$, puis 2 fois $23 \mathrm{~kg} \mathrm{~N} /$ ha urée apporté 2 et 4 semaines après le semis.

\section{Croissance de la culture associée et rendement}

Cette gestion écologique permet l'amélioration de la croissance de la culture, notamment une réduction de la durée du cycle de développement (Balaya et al., 2017). Elle permet également un gain de productivité (biomasse et grains; indice de surface foliaire et indice de végétation normalisé) des cultures annuelles associées à l'arbuste (Dossa et al., 2013; Diakhaté et al., 2013; Debenport et al., 2015; Bright et al., 2017; Bogie et al., 2019).

Dans le cas de $P$. reticulatum à Nioro-du-Rip, les rendements sont significativement augmentés, $\mathrm{y}$ compris pour les systèmes sans apport de fertilisants minéraux : jusqu'à $+137 \%$ en 2013 pour le mil et $+39 \%$ en 2014 pour l'arachide (tab. 12.1; Bright et al., 2017). L'apport d'engrais minéral apparaît même assez discutable. 
Tableau 12.1. Rendements en arachide et en mil cultivés en association avec $P$. reticulatum (+ Pr) ou en absence d'arbuste (- Pr) à Nioro-du-Rip entre 2011 et 2015 (Bright et al., 2017).

\begin{tabular}{|c|c|c|c|c|c|c|c|c|c|c|}
\hline \multirow{3}{*}{$\begin{array}{c}\text { Apports } \\
\text { NPK ( } \times \text { dose } \\
\text { recommandée) }\end{array}$} & \multicolumn{6}{|c|}{$\begin{array}{c}\text { Mil } \\
\text { (rendement en grains kg/ha) }\end{array}$} & \multicolumn{4}{|c|}{$\begin{array}{c}\text { Arachide } \\
\text { (rendement en gousses kg/ha) }\end{array}$} \\
\hline & \multicolumn{2}{|c|}{2011} & \multicolumn{2}{|c|}{2013} & \multicolumn{2}{|c|}{2015} & \multicolumn{2}{|c|}{2012} & \multicolumn{2}{|c|}{2014} \\
\hline & $+\operatorname{Pr}$ & $-\operatorname{Pr}$ & $+\operatorname{Pr}$ & $-\mathrm{Pr}$ & $+\operatorname{Pr}$ & $-\operatorname{Pr}$ & $+\operatorname{Pr}$ & $-\operatorname{Pr}$ & $+\operatorname{Pr}$ & $-P r$ \\
\hline 0 & $886 a$ & $499 \mathrm{~b}$ & $749 a$ & $315 b$ & $592 a$ & $298 \mathrm{a}$ & $1672 \mathrm{a}$ & $1354 \mathrm{a}$ & $1526 a$ & $1096 \mathrm{~b}$ \\
\hline 0,5 & $1429 a$ & $1047 b$ & $1194 a$ & $847 \mathrm{a}$ & $1198 \mathrm{a}$ & $738 \mathrm{~b}$ & $1713 a$ & $1763 a$ & $1380 \mathrm{a}$ & $1204 \mathrm{a}$ \\
\hline 1,0 & $1806 \mathrm{a}$ & $1457 \mathrm{a}$ & $1375 \mathrm{a}$ & $1402 a$ & $1565 \mathrm{a}$ & $1151 b$ & $1710 \mathrm{a}$ & $1837 \mathrm{a}$ & $1237 \mathrm{a}$ & $1137 \mathrm{a}$ \\
\hline 1,5 & $2302 a$ & $1939 a$ & $1675 a$ & $1502 \mathrm{a}$ & $2022 a$ & $1388 b$ & $1812 \mathrm{a}$ & $1794 a$ & $1384 a$ & $1135 a$ \\
\hline
\end{tabular}

Les lettres a et $\mathrm{b}$ indiquent une différence significative au seuil de $5 \%$.

\section{" Une utilisation de l'eau optimisée}

À l'augmentation des rendements, est associée une augmentation de l'efficience d'utilisation de l'eau issue des précipitations (mesurée en $\mathrm{kg}$ de grains/ha/mm de précipitation; Dossa et al., 2013; Bright et al., 2017; Bogie et al., 2019).

Ainsi, à Nioro-du-Rip par exemple, l'association avec $P$. reticulatum a permis en moyenne une augmentation de $36 \%$ de l'efficience d'utilisation de l'eau par la plante cultivée de 2005 à 2015 (Bright et al., 2017). L'humidité du sol décroît avec la profondeur et la distance à l'arbuste. Les teneurs en eau du sol au point de flétrissement sont supérieures sous la touffe arbustive de G. senegalensis (Bogie et al., 2018a). Les mesures effectuées (suivi des variables climatiques, du potentiel hydrique du sol, micro-lysimètres) depuis le début des années 2000 montrent qu'il n'y a pas de compétition pour l'eau entre la plante cultivée et l'arbuste. De plus, l'arbuste favorise la recharge de la nappe en saison des pluies et limite le ruissellement et l'évaporation (Kizito et al., 2006, 2007 et 2012). Des observations complémentaires révèlent la capacité de ces arbustes à redistribuer l'eau dans le sol durant la nuit au profit des horizons de surface, c'est un phénomène de redistribution hydraulique (Kizito et al., 2006, 2007 et 2012). Le potentiel hydrique des sols est modifié selon une périodicité de $24 \mathrm{~h}$ ( $\Psi \mathrm{s}$ de 0,6 à 1,1 MPa), en phase avec l'inversion des flux de sève dans les racines latérales superficielles de l'arbuste entre le jour et la nuit (Kizito et al., 2017).

À Keur Matar, une expérimentation avec marquage isotopique au deutérium de l'eau conduite de début mars à fin mai, en contre-saison (avec irrigation), révèle que l'eau «marquée» prélevée à un mètre de profondeur par les racines de G. senegalensis est redistribuée au profit de la culture associée $(\delta \mathrm{D}>300 \%$ dans les parties aériennes du mil; Bogie et al., 2015. Ghezzehei et al., 2017; Bogie et al., 2018b).

\section{" Une dynamique racinaire particulière}

Les dynamiques racinaires de systèmes associant $G$. senegalensis et le mil ont été suivies entre 2013 et 2015 dans un dispositif expérimental complémentaire utilisant des rhizotrons. Ce dispositif était mis en place par le projet Safse à Keur Matar 
(fig. 12.2; Bright, 2017). Avec un système racinaire se développant plus près de la surface par rapport à celui des arbustes, le mil profite pleinement des précipitations en saison de pluies.

Comme attendu, les taux d'allongement racinaire du G. senegalensis ont été plus élevés en saison humide qu'en saison sèche. Cette différence intersaisonnière a été particulièrement marquée chez les jeunes semis de G. senegalensis et chez les arbustes recépés. De façon inattendue, lorsque les racines du mil se sont développées en saison des pluies, la croissance des racines de l'arbuste associé au mil a été fortement réduite (voire nulle en surface), comparativement à celle observée pour l'arbuste seul. Cependant, cet arrêt de croissance des racines du G. senegalensis est temporaire, un mois seulement, et fait suite à un recépage total de ses parties aériennes intervenu un mois et demi plus tôt (Cournac et al., 2017). Dans l'association culturale, l'arbuste et le mil n'explorent donc pas au même moment les horizons qu'ils exploitent tous les deux dans le profil de sol. Les racines du mil, qui se développent plutôt en surface, tirent profit de cette absence de compétition en ayant accès plus facilement aux éléments nutritifs issus de la minéralisation des résidus restitués à la surface du sol au moment du recépage. Enfin, par le recépage, les agriculteurs réduisent la compétition entre les arbustes et les cultures au moment du semis et, en même temps, ce système favorise la croissance et le rendement des cultures.

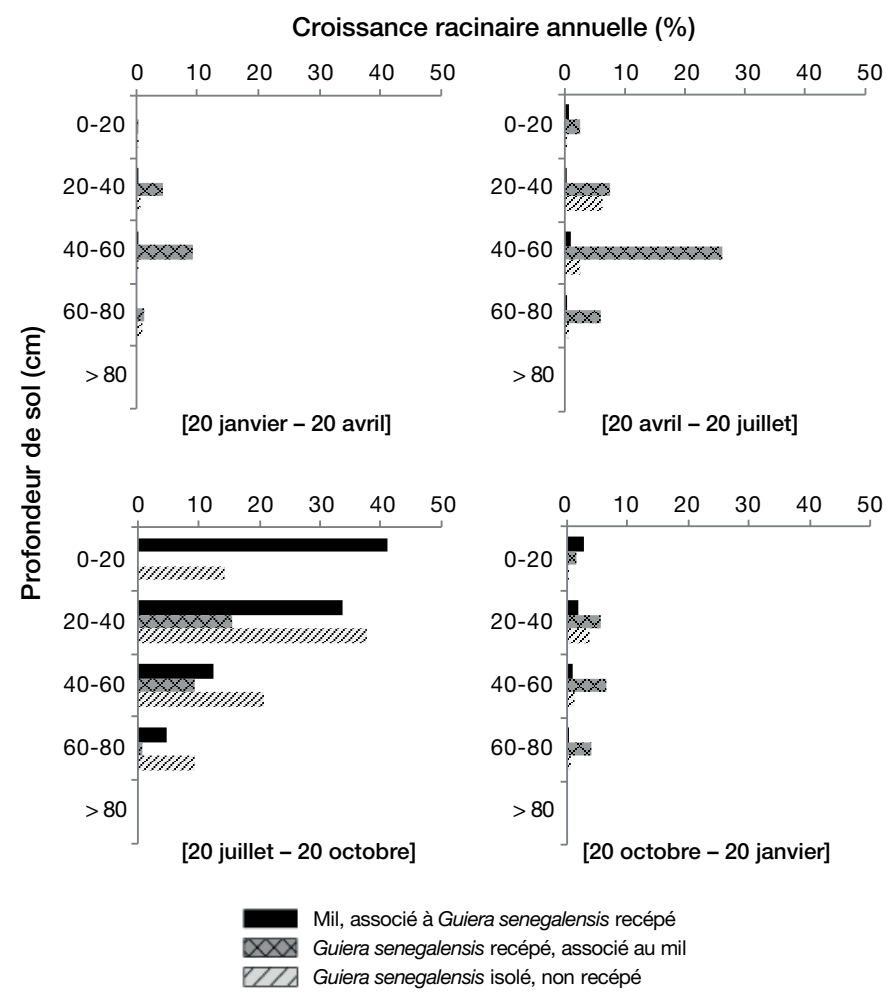

Figure 12.2. Dynamique racinaire moyenne (2013-2015), exprimée en \% de croissance racinaire annuelle sur tout le profil, de touffes de G. senegalensis recépées, du mil poussant à proximité et de touffes isolées et non-recépées $(n=3$, Keur Matar). 


\section{" Un îlot de fertilité}

La gestion, en particulier la taille de l'arbuste dans le système cultivé, permet de préparer un paillis à la surface du sol. Ce paillis, composé des résidus de recépage de l'arbuste, ne limite pas seulement les pertes d'eau. Il constitue une source de carbone et contribue, à travers sa décomposition, à la fourniture de nutriments (Dossa et al., 2009; Diédhiou et al., 2009; Dick et al., 2010). Les plantes cultivées installées à proximité de l'arbuste tirent parti de cet «îlot de fertilité».

Dans les parcelles de mil cultivées en présence de l'arbuste, avec paillis de G. senegalensis à Keur Matar et $P$. reticulatum à Nioro-du-Rip, les teneurs en carbone dans l'horizon superficiel du sol augmentent en moyenne de $11 \%$ (P. reticulatum) à $127 \%$ (G. senegalensis) entre 2012 et 2015 (Bright et al., 2017), notamment au travers des teneurs en matière organique particulaire (fraction de carbone aisément minéralisable de taille $>50 \mu \mathrm{m}$ ). Toutefois, la décomposition et la minéralisation de cette matière organique varient en fonction de la composition des résidus de l'arbuste (rapport C/N, teneurs en polyphénols, lignine, hémicellulose et cellulose; Diédhiou et al., 2009; Diédhiou-Sall et al., 2013). Il est intéressant de noter que les teneurs en nutriments, notamment N, K, Ca et Mg, sont significativement plus élevées dans les sols de l'association culturale, aussi bien en présence de G. senegalensis que de P. reticulatum (Bright et al., 2017). Leffet de la présence des arbustes est aussi notable durant la saison culturale sur les fractions assimilables par la plante (azote minéral et phosphates).

\section{W Un système cultivé favorable aux organismes du sol}

Limportance quantitative et qualitative des communautés microbiennes (bactériennes et fongiques) du sol dans la fourniture des éléments nutritifs pour la plante est connue et largement mentionnée dans la littérature. Nous avons appréhendé cet aspect par diverses méthodes courantes en écologie microbienne des sols.

\section{Diversité et structure des communautés microbiennes du sol}

Lanalyse des phospholipides, composants essentiels des membranes des cellules microbiennes extraits des échantillons de sols sous la forme d'acides gras phospholipidiques (PLFA), a permis de faire une différenciation phénotypique des microorganismes du sol en fonction de la distance à l'arbuste (Diedhiou-Sall et al., 2009). Cette étude a également montré que la biomasse microbienne du sol reste active durant la saison sèche à proximité de l'arbuste (Dick et al., 2017). La structure des communautés microbiennes des échantillons de sol collectés dans les dispositifs de Keur Matar et de Nioro-du-Rip a également été analysée par DGGE

Ces analyses ont permis de discriminer les effets de la présence de l'arbuste et de mettre en valeur le lien entre la structure des communautés microbiennes et certains

1. DGGE ou Denaturing gradient gel electrophoresis, électrophorèse sur gel en gradient dénaturant : c'est une technique de détermination d'empreintes moléculaires des fragments de gènes ribosomiques codant pour l'ADNr 16S pour les communautés bactériennes et l'ADNr 18S ou la région de l'espaceur intergénique transcrit (ITS) pour les communautés fongiques. 
paramètres chimiques du sol, notamment les teneurs en azote minéral (Debenport et al., 2015; Diakhaté et al., 2016).

Une grande biodiversité des communautés bactériennes et fongiques est observée dans la rhizosphère du mil, lorsqu'il est cultivé dans le système agroforestier plutôt qu'en monoculture (Debenport et al., 2015; Diakhaté et al. 2016). Les analyses phylogénétiques permettent de distinguer des taxons mieux adaptés ou plus sensibles aux conditions édaphiques rencontrées dans l'association culturale mil+arbuste pour laquelle les teneurs en carbone et éléments nutritifs dans le sol sont plus élevées (Debenport et al., 2015).

De plus, des plants de mil (50 jours après semis) ont été échantillonnés à proximité $(<1 \mathrm{~m})$ ou à distance $(>5 \mathrm{~m})$ d'un buisson de $G$. senegalensis, le long du gradient climatique nord-sud du bassin arachidier sénégalais (Louga, Bambey et Nioro-duRip). La colonisation des racines de mil par les mycorhizes à arbuscules (AMF) est plus abondante à proximité de l'arbuste, notamment pour les sites du nord, aux sols plus sableux et recevant des précipitations plus faibles (Bright et al., 2014). Une expérimentation en mésocosmes réalisée sous serre a permis de vérifier que la présence de G. senegalensis favorise la croissance du mil par le biais d'un réseau de mycélium partagé favorisant le transfert des nutriments et de l'eau (Dick et al., 2015).

\section{Capacités fonctionnelles des microorganismes}

Les données obtenues sur la structure et la diversité des communautés peuvent être confrontées à d'autres paramètres renseignant sur les capacités fonctionnelles des microorganismes.

Les analyses réalisées sur les échantillons de sols issus des dispositifs expérimentaux de Keur Matar et Nioro-du-Rip entre 2011 et 2016 montrent que dans le sol cultivé en association avec les arbustes, les activités enzymatiques (uréase, arylsulphatase et deshydrogénase) sont plus élevées qu'en culture pure. Elles confirment l'existence d'une activité microbienne et d'un recyclage des nutriments plus importants dans le sol cultivé en association avec les arbustes que dans celui d'une monoculture (Diakhaté et al., 2016).

La méthode MicroResp ${ }^{\mathrm{TM}}$ permet de caractériser l'activité respiratoire des microorganismes après l'apport d'un substrat particulier considéré comme plus ou moins dégradable (Campbell et al., 2003). Pour les échantillons de sols du dispositif de Nioro-du-Rip, les profils d'activité catabolique obtenus par cette méthode révèlent deux aspects intéressants ouvrant de nouvelles pistes de recherche pour la gestion de cet arbuste dans le champ cultivé (Diakhaté et al., 2016) :

- l'apport des résidus de $P$. reticulatum stimule les communautés microbiennes à même de dégrader les composés phénoliques dérivés de la lignine;

- en présence de l'arbuste, les communautés microbiennes du sol dégradent plus efficacement le tréhalose. Ce carbohydrate est impliqué notamment dans l'efficience d'utilisation de l'eau par la plante (Delorge et al., 2014). Il joue également un rôle clé dans les interactions entre la plante et les microorganismes du sol, notamment comme molécule-signal dans le dialogue moléculaire avec les microorganismes symbiotiques ou les pathogènes (Fernandez et al., 2010). 


\section{Les nématodes, indicateurs du fonctionnement biologique du sol}

Du fait de leur abondance dans les sols et de leur diversité fonctionnelle (en lien avec leur régime alimentaire et leur stratégie démographique), les nématodes constituent un autre indicateur de choix permettant d'apprécier les changements du fonctionnement biologique du sol liés aux pratiques agricoles (Bongers et Ferris, 1999).

Dans la monoculture du mil à Nioro-du-Rip, la communauté de nématodes du sol est dominée par les nématodes phytoparasites (60\% de l'abondance totale; Diakhaté et al., 2013), notamment les nématodes spiralés du genre Helicotylenchus ou Scutellonema (Hoplolaimidae). Leur abondance diminue significativement dans l'association culturale, que ce soit en milieu paysan (Diakhaté et al., 2013) ou en conditions contrôlées (Chapuis-Lardy et al., 2015). Labondance de la population de nématodes phytophages est réduite de $64 \%$ quarante-cinq jours après un apport de feuilles ou de rameaux broyés.

La production de composés phénoliques nématicides lors de la dégradation des résidus de plantes ligneuses est un mécanisme suppressif des populations de nématodes phytophages connu pour d'autres espèces de plantes (Ohri et Pannu, 2010). La présence de polyphénols (Dossa et al., 2009), de flavonols et d'oxychromones (Babajide et al., 2008) chez $P$. reticulatum pourrait ainsi être à l'origine de la réduction de l'abondance des nématodes phytophages observée en présence de l'arbuste. Cependant, cette hypothèse reste à confirmer et les quantités à apporter pour maximiser le service de contrôle des nuisibles sans impacter négativement la production de la culture associée restent à déterminer. Il faudra étudier les éventuelles conséquences du potentiel allélopathique ou de l'inhibition de la nitrification, liés à un excès de polyphénols.

Par ailleurs, $P$. reticulatum favorise la prolifération des nématodes bactérivores, notamment les familles Cephalobidae et Rhabditidae (Diakhaté et al., 2013). Cette augmentation de l'abondance relative des nématodes bactérivores se traduit par une valeur plus élevée de l'indice d'enrichissement dans l'association culturale comparativement à la monoculture de mil (Diakhaté et al., 2013). Cet indice fournit des informations sur la disponibilité en ressources dans le sol au travers de la réponse des décomposeurs primaires, notamment les espèces opportunistes (Ferris et al., 2001). Lindice d'enrichissement augmente avec la disponibilité des éléments nutritifs, en particulier l'azote. Ce résultat est en adéquation avec l'augmentation des teneurs en azote minéral observées dans le sol : $8,1 \mathrm{mg} \mathrm{N} / \mathrm{kg}$ de sol de l'association culturale; 4,7 mg N/kg de`sol dans la monoculture de mil (Diakhaté et al., 2013). La dégradation des résidus de l'arbuste contribue sans aucun doute à une augmentation des teneurs en azote minéral. Toutefois, l'abondance des nématodes bactérivores, notamment des Rhabditidae, indique clairement un effet de prédation sur les microorganismes (Bongers et Ferris, 1999) qui conduit au recyclage de l'azote microbien immobilisé (Neher, 2010).

\section{" Vers un modèle d'association de cultures vivrières et d'arbustes natifs adapté au climat sahélien}

Pour les systèmes associant arbustes et cultures annuelles, les travaux menés ces quinze dernières années au Sénégal ont permis de démontrer que la présence des 
arbustes dans l'agrosystème, leur recépage et le maintien des résidus de coupe à la surface du sol permettaient une meilleure efficience d'utilisation de l'eau et la création d'îlots de fertilité favorisant un meilleur rendement des cultures. Ces systèmes abritent des niches écologiques propices au développement de communautés microbiennes (bactéries et champignons) impliquées dans la fourniture d'éléments nutritifs à la culture qui lui est associée. Cette mise à disposition d'éléments nutritifs permet ainsi une meilleure croissance et la régulation de certains bioagresseurs, notamment des nématodes phytophages.

Considérant l'aire de répartition géographique de ces arbustes en zone subsaharienne, l'intensification de leur utilisation dans les champs cultivés devrait permettre d'améliorer la productivité primaire (production de biomasse par unité de temps ou de la production primaire, notamment via la photosynthèse) et le rendement des cultures de manière durable. Pour être proposée, une telle démarche agroécologique doit intégrer les connaissances, les pratiques et les ressources locales. Des travaux ultérieurs devraient permettre d'explorer - dans d'autres contextes pédoclimatiques, agronomiques et socio-économiques, mais aussi au niveau de l'agroécosystème et de l'exploitation agricole - les bénéfices et les inconvénients pour les cultures associées et pour les exploitants (Lahmar et Chapuis-Lardy, 2015).

\section{" Remerciements}

Les travaux ont été menés dès le début des années 2000, grâce au soutien de la National Science fondation (projet $n^{\circ} 0120732$ ), et avec la contribution de nombreux étudiants et chercheurs de 2001 à 2008 : †A. Sène et †M. Khouma (Isra, Sénégal), M. Sène (Isra, Sénégal), S. Diédhiou-Sall (Université de Ziguinchor, Sénégal), A. Samba (Université de Thiès, Sénégal), A. Badiane (Usaid, Sénégal), J. Noller et M. Dragila (Oregon State University, États-Unis), F. Kizito (Ciat, Rwanda), A. Lufafa (Banque mondiale, Ouganda), E. Dossa (IFDC, Ghana).

Les recherches se sont ensuite poursuivies grâce au soutien renouvelé de la National Science fondation (projet nº 0968247 (2010-2017), épaulée par le programme Safse, en impliquant les chercheurs et étudiants co-auteurs du présent chapitre.

\section{" Bibliographie}

Babajide J.O., Babajide O.O., Daramola A.O., Mabusela W.T., 2008. Flavonols and an oxychromonol from Piliostigma reticulatum. Phytochemistry, 69: 2245-2250.

Badiane A.N., Khouma M., Sene M., 2000. Région de Diourbel : gestion des sols. Drylands research working paper 15, Drylands research, Somerset, Rotaume-Uni. www.drylandsresearch.org.uk/pdfs/WP_Badiane_Soils.pdf (consulté le 31 mai 2017).

Balaya R., Bogie N.A., Diedhiou I., Diop A.K., Badji B., Ndiaye M.B., Diallo D., Gaye F., Ndour Badiane Y., 2017. Effect of Guiera senegalensis intercropping on pearl millet ((Pennisetum glaucum (L.) R. Br.) growth and yield in Senegal. In : Shrub intercropping symposium, (Sustainable intensification conference) Conférence intensification durable, 27 avril 2017, Dakar Sénégal.

Bogie N., Bayala R. Diedhiou I., Fogel M., Dick R., Ghezzehei T.A., 2015. Isotopic tracer study of hydraulic transfer between native woody shrubs and associated annual crops under dry conditions in the Sahel. Geophysical research abstract, 17: EGU2015-7609. 
Bogie N.A., Bayala R., Diedhiou I., Dick R.P., Ghezzehei T.A., 2018a. Alteration of soil physical properties and processes after ten years of intercropping with native shrubs in the Sahel. Soil and tillage research, 182: 153-163.

Bogie N.A., Bayala R., Diedhiou I., Dick R.P., Conklin M.H., Fogel M.L., Ghezzehei T.A., 2018b. Hydraulic redistribution by native Sahelian shrubs: Bioirrigation to resist in-season drought. Frontiers in environmental science, 6: 98. doi: 10.3389/fenvs.2018.00098.

Bogie N.A., Bayala R., Diedhiou I., Dick R.P., Ghezzehei T.A., 2019. Intercropping with two native woody shrubs improves water status and development of interplanted groundnut and pearl millet in the Sahel. Plant and soil, 435: 143-159.

Bongers T., Ferris H., 1999. Nematode community structure as a bioindicator in environmental monitoring. Trends in ecology and evolution, 14: 224-228.

Bright M.B.H, Dick R.P., Schreiner P., Mboup Founoune H., Chapuis-Lardy L., 2014. Arbuscular mycorrhizal colonization rate dynamics in a Guiera senegalensis-Millet agroforestry intercropping system across a rainfall gradient of Senegal. In: ASA-CSSA-SSSA International annual meeting, Long Beach (CA, États-Unis), November 2-5. The American society of agronomy, Crop science society of America, and Soil science society of America. Long Beach: ASA-CSSA-SSSA.

Bright M.B.H., Diedhiou I., Balaya R., Assigbetse K., Chapuis-Lardy L., Ndour Y., Dick R.P., 2017. Long-term Piliostigma reticulatum intercropping in the Sahel: crop productivity, carbon sequestration, nutrient cycling, and soil quality. Agriculture, ecosystems and environment, 242: 9-22.

Bright M.B.H., 2017. The role of shrub agroforestry systems in increasing food security for the West African Sahel. Doctor of Philosophy, Ohio State University, Environment and natural resources.

Campbell C.D., Chapman S.J., Cameron C.M., Davidson M.S., Potts J.M., 2003. A rapid microtiter plate method to measure carbon dioxide evolved from carbon substrate amendments so as to determine the physiological profiles of soil microbial communities by using whole soil. Applied and environmental microbiology, 69: 2593-3599.

Chapuis-Lardy L., Diakhaté S., Djigal D., Ba A.O., Dick R.P., Sembène P.M., Masse D., 2015. Potential of Sahelian native shrub materials to suppress the spiral nematode Helicotylenchus dihystera. Journal of nematology, 47: 214-217.

Cournac L., Jourdan C., Moucty Sambou D., Bayala R., Diédhiou I., Bright M., Dick R., 2017. Guiera senegalensis Intercropping with Pennisetum glaucum: temporal and spatial dynamics of shrub and mille. In : Shrub Intercropping symposium, (Sustainable intensification conference) Conférence intensification durable, 27 avril 2017, Dakar, Sénégal.

Debenport S.J., Assigbetse K.B., Bayala R., Chapuis-Lardy L., Dick R.P., McSpadden Gardener B.B., 2015. Shifting populations in the root-zone microbiome of millet associated with enhanced crop productivity in the Sahel. Applied and environmental microbiology, 81: 2841-2851.

Delorge I., Janiak M., Carpentier S., Van Dijck P., 2014. Fine tuning of trehalose biosynthesis and hydrolysis as novel tools for the generation of abiotic stress tolerant plants. Frontiers in plant science, 5: a147.

Diakhaté S., Gueye M., Chevallier T., Diallo N.H., Assigbetse K., Masse D., Sembène M., Ndour Y., Dick R.P., Chapuis-Lardy L., 2016. Soil microbial functional capacity and diversity in a milletshrub intercropping system of semi-arid Senegal. Journal of arid environments, 129: 71-79.

Diakhaté S., Villenave C., Diallo N.H., Ba A.O., Djigal D., Masse D., Sembène P.M., ChapuisLardy L., 2013.The influence of a shrub-based intercropping system on the soil nematofauna when growing millet in Senegal. European journal of soil biology, 57: 37-41. http://csa2015.cirad.fr/var/ csa2015/storage/fckeditor/file/L1\%20Regional\%20Dimensions(1).pdf (consulté le 08/04/2019).

Dick R.P., Diédhiou I., Dossa E., Kizito F., Chapuis-Lardy L., Ndour Badiane Y., Debenport S.J., McSpadden Gardener B.B., Assigbetse K.B., Bright M.B.H., Schreiner P., Founoune Mboup H., Bayala R., Diallo N.H., 2015. Optimizing rhizosphere microbiology and hydrology of shrub-intercropping for buffering climate change in the Sahel. In: $3^{\mathrm{e}}$ Conférence scientifique mondiale sur l'Agriculture Climato-Intelligente, 16-18 mars 2015.

Dick R.P., Diedhiou-Sall S., DeLay C., Assigbetsse K.B., Badiane A., Khouma M., Diedhiou I., Chapuis-Lardy L., Dossa E., 2017. Soil microbial function and profile diversity in the rhizosphere of two indigenous shrubs of the Sahel. In: Shrub intercropping symposium, (Sustainable intensification conference) Conférence intensification durable, 27 avril 2017, Dakar, Sénégal. 
Dick, R., Sene, M., Diack, M., Khouma, M., Badiane, A., Samba, S. A., Diedhiou I., Lufafa A., Dossa E., Kizito F., Diedhiou S., Noller J., Dragila, M. 2010. The native shrubs Piliostigma reticulatum and Guiera senegalensis: the unrecognized potential to remediate degraded soils and optimize productivity of Sahelian agroecosystems. In: Le projet majeur africain de la Grande muraille verte: concepts et mise en ceuvre. Dia A. et Duponnois R., (eds). Paris : Éditions IRD, 199-213. doi :10.4000/books.irdeditions.2126 (consulté le 31 mai 2017).

Diedhiou S., Dossa E.L., Badiane A.N., Diedhiou I., Sene M., Dick R.P., 2009. Decomposition and spatial microbial heterogeneity associated with native shrubs in soils of agroecosystems in semi-arid Senegal. Pedobiologia, 52: 273-286.

Diedhiou-Sall S., Dossa E.L., Badiane A.N., Assigbetse K.B., Diedhiou I., Ndiaye N.A.S., Khouma M., Sène M., Dick R.P, 2013. Microbiology and macrofaunal activity in soil beneath shrub canopies during residue decomposition in agroecosystems of the Sahel. Soil science society of america journal, 77: 501-511.

Dossa E.L., Diedhiou I., Khouma M., Sene M, Badiane A.N., Samba Ndiaye S.A., Assigbetse K.B., Sall S., Lufafa A., Kizito F., Dick R.P., Saxena J., 2013. Crop productivity and nutrient dynamics in a shrub-based farming system of the Sahel. Agronomy journal, 105: 1237-1246.

Dossa E.L., Khouma M., Diedhiou I., Sene M., Kizito F., Badiane A.N., Samba S.A.N., Dick R.P., 2009. Carbon, nitrogen and phosphorus mineralization potential of semiarid Sahelian soils amended with native shrub residues. Geoderma, 148: 251-260.

FAO. 2006. Guidelines for soil description. $4^{\text {th }}$ edition. Rome: FAO.

Fernandez O., Béthencourt L., Quero A., Sangwan R.S., Clément C., 2010. Trehalose and plant stress responses: friend or foe? Trends in plant science, 15: 409-417.

Ferris H., Bongers T., de Goede R.G.M., 2001. A framework for soil food web diagnostics: extension of the nematode faunal analysis concept. Applied soil ecology, 18: 13-29.

Ghezzehei T.A., Dick R.P., Bogie N., Sene M., Kizito F., Dragila M., Diedhiou I., 2017. Intercropped woody species in the Sahel to resist drought: hydraulic lift and crop water relations. In: Shrub intercropping symposium, (Sustainable Intensification Conference) Conférence intensification durable, 27 avril 2017, Dakar, Sénégal.

Hernandez R.R., Debenport S.J., Ndoye F., Nkenmogne I.E.K., Thuita M., Leewis M.C.C.E., Soumare S., Gueye M., Miambi E., Chapuis-Lardy L., Diedhiou I., Dick R.P., 2015. The native shrub, Piliostigma reticulatum, as an ecological resource island for mango trees in the Sahel. Agriculture, ecosystem and environment, 204: 51-61.

Kizito F., Draglia M., Sene M., Lufafa A., Diedhiou I., Dick R.P., Selker J.S., Diack M., Dossa E., Khouma M., Badiane A.N., Ndiaye S., 2006. Seasonal soil water variation and root patters between two semi-arid shrubs co-existing with pearl millet in Senegal, West Africa. Journal of arid environments, 67: 436-455.

Kizito F., Sene M., Draglia M., Lufafa A., Diedhiou I., Dossa E., Cuenca R., Selker J.S., Dick R.P., 2007. Soil water balance of annual crop-native shrub systems in Senegal's peanut basin: the missing link. Agriculture and water management, 90: 137-148.

Kizito F., Sene M., Ghezzehei T.A., Dick R.P., Bogie N., Diedhiou I., Dragila M., 2017. Shrub-crop hydrology: water balance, competition and hydraulic redistribution. In : Shrub Intercropping symposium, (Sustainable Intensification Conference) Conférence intensification durable, 27 avril 2017, Dakar, Sénégal.

Kizito F., Dragila M.I., Sene M., Brooks R.J., Meinzer F.C., Diedhiou I., Diouf M., Lufafa A., Dick R.P., Selker J., Cuenca R.H, 2012. Hydraulic redistribution by two semi-arid shrub species: implications for sahelian Agro-ecosystems. Journal of arid environments 83: 69-77.

Lahmar R., Bationo B.A., Lamso N.D., Guero Y., Tittonell P., 2012. Tailoring conservation agriculture technologies to West-Africa semi-arid zones: building on traditional local practices for soil restoration. Field crops research, 132: 158-167.

Lahmar R., Chapuis-Lardy L., 2015. Les arbustes natifs dans les champs cultivés : un atout pour le développement de systèmes agroécologiques innovants et efficients dans les zones sèches de l'Afrique. In: Dossier thématique du CSFD n 11, Masse D., Chotte J. L., Scopel E., (eds). Montpellier : Comité scientifique français de la désertification CSFD, Agropolis Fondation, 19-21. 
Masse D., Ndour Badiane Y., Hien E., Akpo L.E., Assigbetsé K., Bilgo A., Diédhiou I., Hien V., Chapuis-Lardy L., 2013. Lagriculture africaine face aux changements globaux : recherches et innovations basées sur les sciences de l'écologie. Comptes rendus biologies, 336 : 289-294.

Neher D.A., 2010. Ecology of plant and free-living nematodes in natural and agricultural soil. Annual reviews in phytopathology, 48: 371-394.

Ohri P., Pannu S.K., 2010. Effect of phenolic compounds on nematodes - A review. Journal of applied and natural science, 2: 344-350.

Seneviratne S.I., Nicholls N., Easterling D., Goodess C.M., Kanae S., Kossin J., Luo Y., Marengo J., McInnes K., Rahimi M., Reichstein M., Sorteberg A., Vera C., Zhang X., 2012. Changes in climate extremes and their impacts on the natural physical environment. In: Managing the risks of extreme events and disasters to advance climate change adaptation, Field, C.B., V. Barros, T.F. Stocker, et al. (eds). A Special report of working groups I and II of the Intergovernmental panel on climate change (IPCC). Cambridge University Press, Cambridge, Royaume-Uni, and New York, NY, États-Unis, 109-230.

Yelemou B., Bationo B.A., Yameogo G., Millogo-Rasolodimby J., 2007. Gestion traditionnelle et usages de Piliostigma reticulatum sur le plateau central du Burkina Faso. Bois et forêts des tropiques, $291: 55-66$. 


\title{
Contribution des arbustes au fonctionnement hydrique et carboné des parcs agroforestiers à Guiera senegalensis J.F. Gmel : observations et modélisation
}

\author{
Issoufou Bil-Assanou H., Demarty J., Cappelaere B., \\ Allies A., Velluet C., Maïnassara I., Oï M., Chazarin J.-P., \\ Moussa Moumouni R. et Seghieri J.
}

Résumé. Notre objectif est d'évaluer la contribution des arbustes de Guiera senegalensis aux flux d'eau et de carbone dans un parc agroforestier à petit mil (Pennisetum glaucum L.R. Br.) et dans une jachère. Le site expérimental est situé au sud-ouest du Niger, sur un bassin-versant sahélien de $2 \mathrm{~km}^{2}$. À l'échelle de l'arbuste de Guiera senegalensis, le taux de transpiration foliaire a été déduit de la conductance stomatique et du déficit de pression de vapeur. La dynamique de la biomasse aérienne et souterraine des arbustes a été suivie en saison des pluies, en saison sèche froide et en saison sèche chaude. À l'échelle de la parcelle, les bilans d'eau et d'assimilation du carbone ont été estimés par une modélisation de type «transferts surface-végétation-atmosphère». Le modèle est paramétré à partir de mesures automatiques de terrain selon la méthode des corrélations turbulentes. Le taux de transpiration foliaire et la biomasse aérienne de Guiera ont augmenté dans le parc, de la saison des pluies à la saison sèche chaude, alors qu'ils ont diminué dans la jachère. Les résultats du modèle montrent une activité de la jachère centrée sur la saison des pluies, mais décalée vers le début de la saison sèche pour le parc. À l'échelle de la parcelle, le modèle est capable de bien simuler l'évapotranspiration et l'assimilation de carbone au regard de la période de la croissance active des arbustes dans les deux types de couvert, tout en assurant un haut degré de cohérence avec le contenu en eau du sol.

Abstract. The main objective of this work is to evaluate the contribution of the shrubs to the water and $\mathrm{CO}_{2}$ flux in the soil-vegetation-atmosphere continuum and to the carbon sequestration in a pearl millet-Guiera senegalensis agroforestry parkland and a fallow. The experimental site is located in a $2 \mathrm{~km}^{2}$ Sahelian watershed in South-West Niger. At 
shrub scale, the leaf transpiration rate was deduced from stomatal conductance and vapor pressure deficit. The dynamics of field above- and below-ground biomass dynamic survey was conducted in rainy, cool and hot dry seasons. At plot scale, water budget and carbon assimilation were estimated by Svat (surface-vegetation-atmosphere transfer) modeling approach, parameterized from field measurements (Eddy correlation). At shrub scale, leaf transpiration rate and aboveground biomass increased in the parkland from rainy to hot dry season while it decreased in fallow. The model results show a fallow activity peaking during the rainy season but shifting towards the beginning of the dry season for the parkland. At plot scale, the model is able to finely match the measured evapotranspiration and carbon assimilation with regard to the period of active growth of shrubs specific, keeping consistent with the soil water in both cover types.

\section{"Introduction}

Lassociation culture-arbuste est typique des systèmes d'agriculture sahéliens. Elle est étudiée depuis plus de vingt ans (Louppe, 1991; Wezel et al., 2000; voir également chapitre 12). Guiera senegalensis J.F. Gmel est une Combretaceae soudano-sahélienne dominante sur les sols sableux, lessivés ou épuisés. En zone sahélienne agropastorale en particulier, malgré la forte pression de coupe exercée par les agriculteurs, l'espèce se maintient dans les parcs agroforestiers en association avec le petit mil (Pennisetum glaucum) et, par conséquent, aussi dans les jachères (Saâdou, 1990; Arbonnier, 2001). Dans les champs de mil, les arbustes sont rabattus au moment du semis ou de la levée des plantules de mil pour éliminer toute compétition avec la céréale. De plus, le bois est utilisé comme combustible (Louppe, 1991).

En pleine saison des pluies, lorsque la compétition pour l'eau et les nutriments n'est plus préjudiciable à la croissance du mil, la partie aérienne des arbustes se régénère alors de façon rapide, de sorte que les plants de mil et les rejets de G. senegalensis coexistent jusqu'à la récolte du mil, en fin de saison des pluies. Les rejets conservent leur feuillage durant presque toute la saison sèche, contrairement aux arbustes non rabattus de la jachère. Nos premiers résultats (Issoufou et al., 2013) ont montré que le maintien du feuillage était dû à un stress hydrique relativement faible des rejets (potentiel hydrique foliaire faiblement négatif) du fait d'une conductance hydraulique élevée et d'une embolie initiale faible dans les vaisseaux encore jeunes des rejets. Labsence de stress a été interprétée comme une probable conséquence d'un ratio racines/partie aérienne plus élevé chez les rejets que chez les arbustes non rabattus en jachère. De plus, la densité des arbustes est plus faible dans les parcs agroforestiers que dans les jachères, ce qui induit une plus grande disponibilité en eau pour les arbustes dans les parcs (Ramier et al., 2009). Enfin, la gestion des champs pour une production maximale de mil bénéficierait aussi aux rejets; certaines études récentes ont montré qu'une augmentation des rendements céréaliers autour des arbustes était liée à un effet d'«îlot de fertilité» de l'arbuste (Wezel, 2000; Wezel et al., 2000, voir chapitre 12).

Dans ce chapitre, le fonctionnement éco-hydrologique du parc agroforestier et de la jachère est étudié sous l'angle des processus d'échange d'eau et de carbone dans le continuum sol-végétation-atmosphère, à partir des observations in situ à l'échelle de l'arbuste et de la parcelle et des développements récents d'une modélisation de type 
«transferts surface-végétation-atmosphère» (TSVA). Les résultats sont présentés en distinguant, d'une part, les processus de régulation hydrique et, d'autre part, les processus d'assimilation et de séquestration du carbone.

\section{" Site d'étude et méthodologie}

Un parc agroforestier à G. senegalensis et une jachère ont été instrumentés en 2005 pour des mesures intensives et un suivi sur le long terme sur le versant de Wankama $\left(2,6^{\circ} \mathrm{E} ; 13,6^{\circ} \mathrm{N}\right)$, à environ $60 \mathrm{~km}$ à l'est de Niamey au Niger (Cappelaere et al., 2009). Ce versant est l'un des sites pilote du Service national d'Observation (SNO) et d'analyse multidisciplinaire de la mousson africaine - couplage de l'atmosphère tropicale et du cycle hydrologique (Amma-Catch, 2018). Un des objectifs de cet observatoire est d'étudier les interactions et les couplages entre les cycles de l'énergie, de l'eau et du carbone à partir des mesures de processus microclimatiques et de la végétation. Des mesures et des observations sont réalisées conjointement sur une parcelle située dans un parc agroforestier associant le petit mil et $G$. senegalensis et sur une parcelle située dans une jachère (savane arbustive). Elles ont permis de mesurer et de caractériser :

- la pluviométrie et la micro-météorologie locales;

- les échanges thermo-hydriques dans le continuum sol-végétation-atmosphère à l'échelle de la parcelle (humidimétrie et thermique du sol, flux d'énergie, d'eau et de $\mathrm{CO}_{2}$ à l'interface surface-atmosphère);

- le développement saisonnier du couvert (hauteur et indice foliaire) et sa productivité (biomasses aérienne et souterraine, opération financée par le projet Safse, 2018).

À l'échelle de l'arbuste, le taux de transpiration foliaire $\left(\mathrm{Tr}, \mathrm{mmol} / \mathrm{m}^{2} / \mathrm{s}\right)$ a été déduit à partir de la conductance stomatique et du déficit de pression de la vapeur par Issoufou et al. (2013) dans le parc agroforestier et dans la jachère. Pour compléter cette étude, dix-huit arbustes espacés d'au moins $3 \mathrm{~m}$ les uns des autres ont été échantillonnés dans le parc agroforestier et dans la jachère. Leur hauteur totale, le plus grand diamètre de houppier et le diamètre perpendiculaire (généralement le plus petit) ont été mesurés. Les biomasses aérienne et souterraine de chaque arbuste ont été estimées par la méthode destructive et l'excavation exhaustive du système souterrain jusqu'à $1 \mathrm{~m}$ de profondeur. En effet, l'arbuste possèderait plus de $90 \%$ de sa biomasse racinaire dans cet horizon (Kizito et al., 2007). Ce protocole a été appliqué en saison des pluies (juin-octobre), en saison sèche froide (novembre-janvier) et en saison sèche chaude (février-mai). Les biomasses ont été ensuite estimées (au laboratoire de l'IRD de Niamey) par pesée après étuvage, à $70^{\circ} \mathrm{C}$ pour les feuilles et les racines fines et à $105^{\circ} \mathrm{C}$ pour le bois et les racines architecturales. Le carbone total contenu dans les arbustes est estimé à $50 \%$ de la biomasse sèche selon les références sur les espèces ligneuses de savanes de IPCC (2007) et Lufafa et al. (2008).

À l'échelle de la parcelle, une modélisation des transferts sol-végétation-atmosphère est développée. Elle est adaptée au milieu sahélien (modèle Sispat - Simple soil plant atmosphere transfer; Braud et al., 1995; Braud, 1998) et repose sur les observations faites dans le cadre du Service national d'observation (Amma-Catch). Une étape préliminaire a consisté à éprouver la capacité de ce modèle à décrire le 
fonctionnement thermo-hydrique du parc agroforestier (Velluet et al., 2014). Des développements récents ont ensuite permis la prise en compte du fonctionnement carboné du parc agroforestier et de la jachère par l'intégration d'un module d'assimilation photosynthétique et de régulation stomatique (modèle A-gs; Jacobs, 1994) au modèle initial des transferts sol-végétation-atmosphère. La capacité du nouveau modèle à estimer les flux de $\mathrm{CO}_{2}$ à l'interface sol-végétation-atmosphère, tout en conservant sa robustesse sur les flux et les stocks d'eau et d'énergie, a été évaluée (Allies, 2015).

\section{" Régulation hydrique}

\section{Régulation hydrique à l'échelle de l'arbre}

La fig. 13.1 présente la pluviométrie journalière, le déficit de pression de vapeur (DPV) moyen journalier et le taux de transpiration foliaire journalière maximale (Tr max) mesurés au cours en 2010. Le taux de transpiration foliaire ( $\operatorname{Tr} \max$ ) est corrélé positivement au déficit de pression de vapeur dans le parc agroforestier uniquement $(\mathrm{F}=6,461 ; \mathrm{P}=0,03856)$. La transpiration foliaire diminue concomitamment avec le déficit de pression de vapeur de juillet à septembre, les mois les plus pluvieux de l'année. Les mesures de terrain et les sorties du modèle Sispat (fig. 13.2) confirment qu'en cette saison, la disponibilité en eau du sol pour la végétation est plus élevée sous le parc à G. senegalensis que sous la jachère (Ramier et al., 2009; Velluet et al., 2014; Issoufou et al., 2015). À l'échelle de l'arbre, ces résultats indiquent que, tant que le contenu en eau du sol est suffisant pour compenser la transpiration du couvert ligneux, le taux maximum de transpiration du couvert ligneux est limité par la demande évaporative de l'atmosphère, comme l'est l'évapotranspiration à l'échelle de la parcelle. Boulain et al. (2009) ont en effet montré que, dans les parcelles localisées dans le parc agroforestier, l'évapotranspiration était limitée par les conditions atmosphériques jusqu'à un seuil de teneur relative en eau du sol correspondant à $70 \%$. En dessous de ce seuil, elle diminue avec la baisse de la teneur en eau du sol.

\section{Régulation hydrique à l'échelle de la parcelle}

Les comportements hydrologiques et énergétiques du parc agroforestier et de la jachère à l'échelle de la parcelle ont été modélisés de 2005 à 2012 à partir des observations de terrain : précipitations, rayonnements, micro-météorologie, humidimétrie et développement saisonnier de la végétation. La figure 13.2 illustre les évolutions saisonnières annuelles moyennes des composantes du cycle hydrologique simulées par le modèle Sispat dans le parc agroforestier et en jachère.

Des différences de fonctionnement apparaissent clairement entre les deux types de couverts, alors qu'ils sont soumis à des conditions pluviométriques et météorologiques similaires. Le ruissellement et l'évaporation directe du sol sont plus faibles dans le parc agroforestier que dans la jachère, tandis que le stockage de l'eau dans la zone racinaire y est plus élevé. Le pic de la transpiration foliaire (Tr max) est également plus tardif (octobre) dans le parc agroforestier par rapport 
à la jachère (septembre); ce décalage s'explique principalement par la reprise de la régénération intensive des rejets dans le parc agroforestier après la récolte du mil (Issoufou et al., 2013, 2015). La régénération des arbustes est favorisée par les plus grandes quantités d'eau disponibles, une compétition pour les ressources (en particulier l'eau) limitée par un couvert moins dense qu'en jachère et la diminution de l'encroûtement du sol par le travail du sol effectué auparavant pour le mil. On observe une plus grande capacité du parc agroforestier à stocker ou à remo-

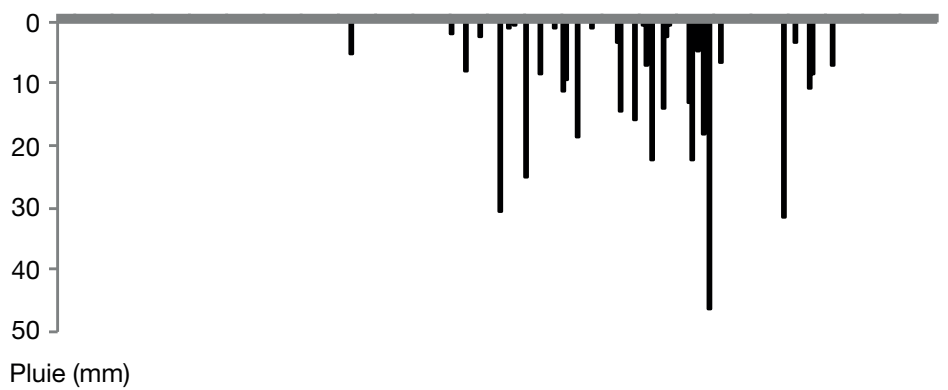

Figure 13.1a. Variation de la pluviométrie en 2010.

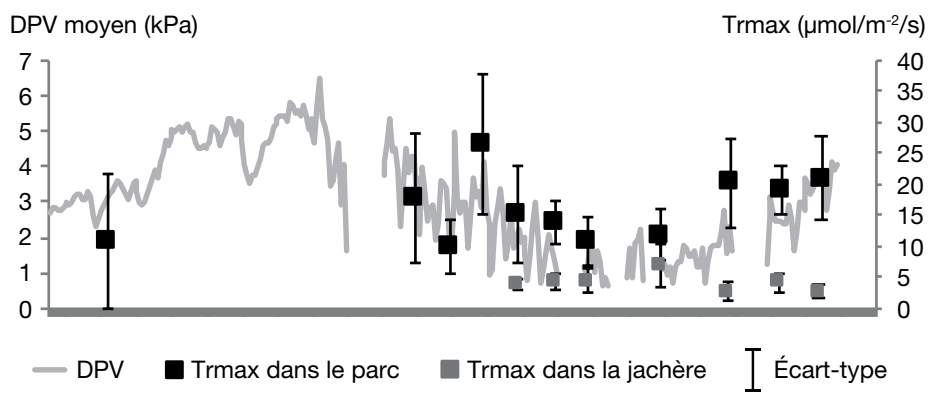

Figure 13.1b. Variations du déficit de pression de vapeur (DPV) et du taux maximum de transpiration journalier (Tr max) mesurée chez G. senegalensis

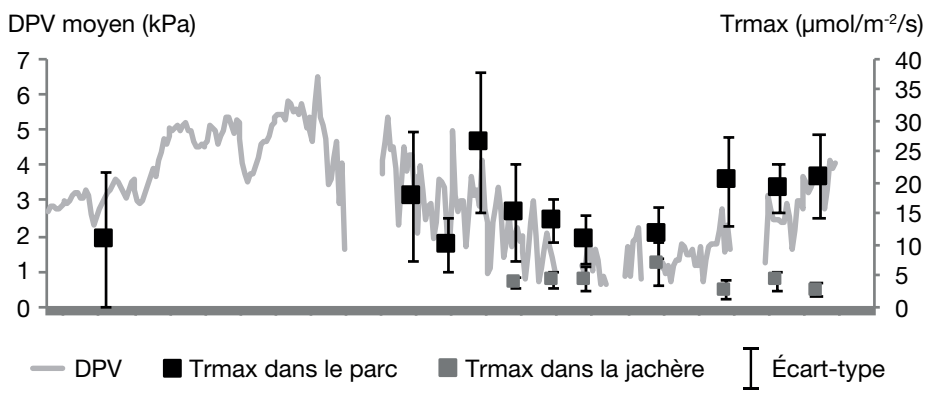

Figure 13.2. Bilan hydrique simulé par le modèle Sispat dans le parc agroforestier et dans la jachère.

Variations saisonnières moyennes simulées des composantes du cycle de l'eau, pour les jachères (lignes continues) et les parcelles en parc agroforestier associant petit mil et Guiera senegalensis (lignes pointillées), exprimées en moyenne glissante de 10 jours simulées pour sept années d'observation, de 2005 à 2012 (Velluet et al., 2014, modifié). 
biliser de l'eau dans la zone racinaire et au-delà (drainage intense sous le parc mais inexistant sous la jachère; fig. 13.2) qui pourrait résulter d'une contribution des parcs agroforestiers à la recharge diffuse de la nappe (Massuel et al., 2011; Ibrahim et al., 2014).

\section{" Stock et assimilation de carbone \\ Évaluation de la biomasse et du stock de carbone à l'échelle de l'arbre}

La gestion des arbustes dans les parcs arbustifs d'Afrique de l'Ouest est liée au calendrier cultural et aux stades de développement de la culture (Lahmar et al., 2012; Issoufou et al., 2015). Dans le parc à $G$. senegalensis et dans la jachère, nous avons quantifié la biomasse sèche totale produite par arbuste au cours de la saison des pluies, la saison sèche froide et la saison sèche chaude (tab. 13.1). La biomasse totale a augmenté de $13 \%$ dans le parc agroforestier et diminué de $15 \%$ dans la jachère entre la saison des pluies et la saison sèche chaude (février-mai) avant défrichement.

Dans le parc agroforestier, l'augmentation de la biomasse totale est due à un accroissement de la biomasse aérienne de $55 \%$ et une réduction de la biomasse racinaire de $16 \%$, principalement les racines fines; le ratio biomasse racinaire/biomasse aérienne a diminué de moitié dans le même temps (de 3 à 1,5). Dans le parc agroforestier, une diminution significative de la quantité de racines fines de $30 \%$ en saison sèche froide et de $45 \%$ en saison sèche chaude est observée également.

Du fait de la défeuillaison et du ralentissement de la croissance des arbustes de février à juin dans la jachère (Issoufou et al., 2013), la diminution de la biomasse est plus liée à une réduction de la biomasse racinaire (19\% pour les racines grossières et $40 \%$ pour les racines fines) qu'à celle de la biomasse aérienne (10\%).

Ainsi, dans la jachère, le ratio biomasse racinaire/biomasse aérienne est passé de 1 en saison des pluies à 0,85 en saison sèche froide et 0,94 en saison sèche chaude. Cette dynamique intersaisonnière de la biomasse ligneuse correspond à une augmentation de stock de carbone de $13 \%$ dans le parc agroforestier et une diminution de $15 \%$ dans la jachère entre la saison des pluies et la saison sèche chaude (tab. 13.2).

Dans le parc agroforestier, la biomasse arbustive (3,5-3,9 tDM/h) et le stock de carbone (1,7-1,9 tC/ha) se situent dans la gamme de valeurs proches de celles $(0,44-$ 4,58 tDM/ha et 0,9-1,4 tC/ha respectivement) observée par Lufafa et al. $(2008,2009)$ dans un parc similaire au Sénégal avec une pluviométrie de $540 \mathrm{~mm} / \mathrm{an}$. Le système racinaire arbustif a stocké $75 \%$ du carbone total en saison des pluies et $56 \%$ en saison sèche chaude dans le parc agroforestier, contre $50 \%$ pour la jachère sur les mêmes périodes.

\section{Assimilation de carbone à l'échelle de la parcelle}

Les processus de photosynthèse et de transpiration sont couplés à l'échelle de la feuille par le biais de la régulation stomatique. Le couplage réalisé entre le modèle Sispat et le module de photosynthèse de Jacobs (1994) permet désormais de tenir 
Tableau 13.1. Variation de la biomasse aérienne et souterraine moyennes en fonction des saisons chez $G$. senegalensis dans le parc agroforestier et dans la jachère du bassinversant à Wankama, Niger.

\begin{tabular}{|c|c|c|c|c|c|}
\hline \multirow[b]{2}{*}{ Parcelle } & \multirow[b]{2}{*}{ Période } & \multicolumn{2}{|c|}{ Biomasse racinaire (tDM/ha) } & \multirow[b]{2}{*}{$\begin{array}{l}\text { Biomasse } \\
\text { aérienne } \\
\text { (tDM/ha) }\end{array}$} & \multirow[b]{2}{*}{$\begin{array}{l}\text { Biomasse } \\
\text { totale } \\
\text { (tDM/ha) }\end{array}$} \\
\hline & & $\begin{array}{l}\text { Racines } \\
\text { grossières } \\
>2 \mathrm{~mm}\end{array}$ & $\begin{array}{c}\text { Racines } \\
\text { nourricières } \\
<2 \mathrm{~mm}\end{array}$ & & \\
\hline \multirow{3}{*}{$\begin{array}{l}\text { Parc } \\
\text { agroforestier }\end{array}$} & $\begin{array}{l}\text { Saison des pluies } \\
(n=6)^{(1)}\end{array}$ & $2,66(0,27)^{(2)}$ & $0,11(0,026)$ & $0,75(0,07)$ & $3,52(0,28)$ \\
\hline & $\begin{array}{l}\text { Saison sèche froide } \\
(\mathrm{n}=6)\end{array}$ & $2,37(0,45)$ & $0,07(0,02)$ & $1,40(0,17)$ & $3,83(0,45)$ \\
\hline & $\begin{array}{l}\text { Saison sèche chaude } \\
(\mathrm{n}=6)\end{array}$ & $2,23(0,61)$ & $0,05(0,01)$ & $1,70(0.14)$ & $3,98(0,63)$ \\
\hline \multirow{3}{*}{ Jachère } & $\begin{array}{l}\text { Saison des pluies } \\
(n=6)\end{array}$ & $4,19(0,75)$ & $0,13(0,02)$ & $4,12(0,62)$ & $8,43(0,69)$ \\
\hline & $\begin{array}{l}\text { Saison sèche froide } \\
(\mathrm{n}=6)\end{array}$ & $3,57(1,14)$ & $0,01(0,02)$ & $4,29(0,57)$ & $7,96(1,38)$ \\
\hline & $\begin{array}{l}\text { Saison sèche chaude } \\
(\mathrm{n}=6)\end{array}$ & $3,39(0,33)$ & $0,07(0,02)$ & $3,68(0,93)$ & $7,14(1,04)$ \\
\hline
\end{tabular}

(1) $\mathrm{n}$ : nombre d'arbustes échantillonnés. (2) Écart-type de la moyenne.

Tableau 13.2. Taux moyen du stock de carbone dans la biomasse aérienne et souterraine en fonction des saisons chez l'arbuste $G$. senegalensis dans le parc agroforestier et la jachère à Wankama, Niger.

\begin{tabular}{lllcc}
\hline Parcelle & \multicolumn{1}{c}{ Période } & $\begin{array}{c}\text { Carbone de la } \\
\text { biomasse racinaire } \\
(\mathbf{t C} / \mathbf{h a})\end{array}$ & $\begin{array}{c}\text { Carbone de la } \\
\text { biomasse aérienne } \\
\mathbf{( t C / h a )}\end{array}$ & $\begin{array}{c}\text { Carbone total de la } \\
\text { biomasse (tC/ha) }\end{array}$ \\
\hline \multirow{2}{*}{ Parc } & $\begin{array}{l}\text { Saison des pluies } \\
(\mathrm{n}=6)^{(1)}\end{array}$ & $1,33(0,13)^{(2)}$ & $0,38(0,036)$ & $1,76(0,14)$ \\
\cline { 2 - 5 } & $\begin{array}{l}\text { Saison sèche } \\
\text { froide (n=6) }\end{array}$ & $1,18(0,22)$ & $0,70(0,08)$ & $1,92(0,23)$ \\
\cline { 2 - 5 } & $\begin{array}{l}\text { Saison sèche } \\
\text { chaude (n=6) }\end{array}$ & $1,12(0,30)$ & $0,85(0,07)$ & $1,99(0,32)$ \\
\hline \multirow{3}{*}{ Jachère } & $\begin{array}{l}\text { Saison des pluies } \\
\text { (n=6) }\end{array}$ & $2,09(0,37)$ & $2,06(0,31)$ & $4,22(0,34)$ \\
\cline { 2 - 5 } & $\begin{array}{l}\text { Saison sèche } \\
\text { froide }(\mathrm{n}=6)\end{array}$ & $1,79(0,57)$ & $2,15(0,28)$ & $3,98(0,69)$ \\
\cline { 2 - 5 } & $\begin{array}{l}\text { Saison sèche } \\
\text { chaude }(\mathrm{n}=6)\end{array}$ & $1,69(0,17)$ & $1,84(0,46)$ & $3,57(0,52)$ \\
\hline
\end{tabular}

(1) $\mathrm{n}$ : nombre d'arbustes échantillonnés. (2) Écart-type de la moyenne. 
compte de ces processus de manière mécaniste dans la modélisation des transferts sol-végétation-atmosphère. Il permet aussi de comprendre le fonctionnement de l'assimilation du carbone atmosphérique par les feuilles, qui peut être ensuite comparée aux observations (Allies, 2015). Les résultats ont montré la bonne tenue du modèle couplé à restituer le cycle annuel de photosynthèse observé dans le parc agroforestier et la jachère (fig. 13.3).

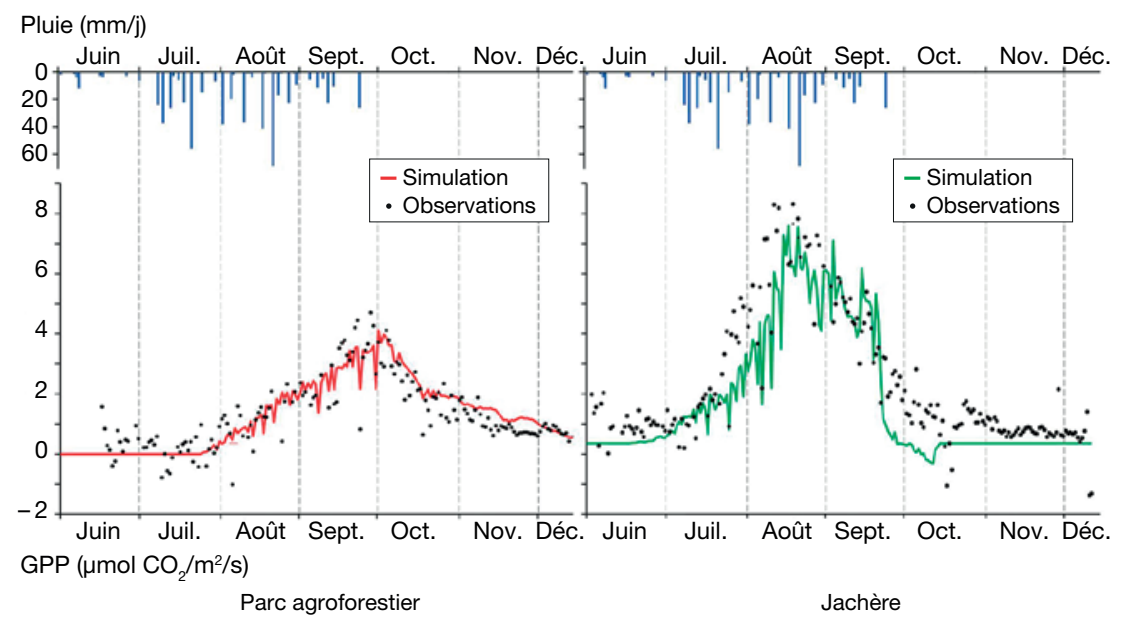

Figure 13.3. Pluviométrie et variations saisonnières de l'assimilation de carbone (Gross Primary Productivity - micromoles $\mathrm{CO}_{2} / \mathrm{m}^{2} / \mathrm{s}$ ) observées et simulées pour le parc agroforestier associant mil-Guiera senegalensis et la jachère en 2007.

Précisément, les différences obtenues entre le modèle et les observations sont plus faibles pour le parc agroforestier que pour la jachère, ceci pouvant s'expliquer par une composition spécifique simple (mil et rejets d'arbustes du même âge) et un couvert (plants de mil et repousses de Guiera) plus homogène dans le parc agroforestier que dans la jachère. En effet, la jachère se compose d'un mélange d'arbustes (photosynthèse en $\mathrm{C}_{3}$ ) d'âges différents avec des plantes annuelles (photosynthèse en $\mathrm{C}_{3}$ et $\mathrm{C}_{4}$ ) qui ne sont pas différenciées dans le modèle actuel.

Les évolutions saisonnières du couvert du parc agroforestier et de la jachère sont contrastées : pour la jachère, un départ précoce, un phasage net avec la saison des pluies et un cycle d'assimilation de carbone raccourci. De telles différences sont directement liées aux résultats obtenus concernant la régulation hydrique. Elles sont dues aussi aux effets du défrichage du parc agroforestier en début de saison, au semis du mil après les premières pluies, à la reprise de la régénération intensive des rejets d'arbustes au cour de saison des pluies et son maintien tout au long de la saison sèche après la récolte du mil.

\section{Conclusion}

Dans les parcs agroforestiers, la contribution de la transpiration des rejets de G. senegalensis à l'évapotranspiration réelle est significative après la récolte de mil, c'est-à-dire à partir de la fin de la saison des pluies. La régénération intensive des arbustes est liée 
à leurs caractéristiques physiologiques propres, elle est favorisée par des conditions de plus grande disponibilité en eau que dans les jachères. Nos résultats montrent la meilleure capacité des rejets à séquestrer du carbone en saison sèche que celle des arbustes matures en jachère, qui sont partiellement défeuillés et très peu actifs. Ces résultats mettent également en évidence une réduction de la biomasse racinaire des arbustes dans le parc agroforestier et dans la jachère en saison sèche. Toutefois, la version actuelle du modèle des transferts sol-végétation-atmosphère fonctionne sur un forçage des paramètres liés à la végétation (i.e. profil racinaire et indice foliaire). Létape suivante serait d'inclure d'un module d'allocation du carbone aux différents organes de la plante (G. senegalensis en jachère, mil et G. senegalensis en parc agroforestier). Ce type de dispositif de mesures envisagé à l'échelle de la plante et de la parcelle, ainsi que la modélisation associée, permettront d'aborder des études d'impacts des différents scénarios de réaffectation d'usage des terres et de changement climatique sur le devenir des ressources hydrologiques et végétales.

\section{Remerciements}

Ce travail a été réalisé en partie grâce au financement du Système national d'observation «Analyse multidisciplinaire de la mousson africaine : couplage de l'atmosphère tropicale et du cycle hydrologique» et du projet Cirad-IRD Safse. Lacquisition et le traitement des données ont été assurés par l'équipe du laboratoire de l'IRD Niamey et celle de l'Université Dan Dicko Dankoulodo de Maradi.

\section{" Bibliographie}

Allies A., 2015. Étude des interactions hydrologie-végétation en milieu sahélien : apport d'un nouveau modèle de surface et impacts des pratiques agropastorales sur les ressources. Mémoire de master mention Eau (Spécialité Hydrologie risque environnement). Montpellier : Université de Montpellier, $127 \mathrm{p}$.

Amma-Catch, 2018. Analyse multidisciplinaire de la mousson africaine : couplage de l'atmosphère tropicale et du cycle hydrologique. www.amma-catch.org/ (Consulté le 31 mars 2017)

Arbonnier M., 2011. Arbres, arbustes et lianes des zones sèches d'Afrique de l'Ouest. $3^{\mathrm{e}}$ ed. Versailles, France : Éditions Quæ.

Boulain N., Cappelaere B., Ramier D., Issoufou H.B.A., Halilou O., Seghieri J., Guillemin F., Oï M., Gignoux J., Timouk F., 2009. Towards an understanding of coupled physical and biological processes in cultivated Sahel. 2. Vegetation and carbon dynamics. Journal of hydrology, 375: 190-203.

Braud I., Dantas-Antonino A.C., Vauclin M., Thony J.L., Ruelle P., 1995. A simple soil-plantatmosphere transfer model (Sispat) development and field verification. Journal of hydrology, 166: 213-250.

Braud I., 1998. Spatial variability of surface properties and estimation of surface fluxes of a savannah. Agricultural and forest meteorology, 89: 15-44.

Cappelaere B., Descroix L., Lebel T., Boulain N., Ramier D., Laurent J.P., Favreau G., Boubkraoui S., Boucher M., Bouzou Moussa I., Chaffard V., Hiernaux P., Issoufou H.B.A., Le Breton E., Mamadou I., Nazoumou Y., Oi M., Ottle C., Quantin G., 2009. The Amma-Catch experiment in the cultivated sahelian area of southwest Niger-investigating water cycle response to a fluctuating climate and changing environment. Journal of hydrology, 375: 34-51. 
Ibrahim M., Favreau G., Scanlon B.R., Seidel J.L., Le Coz M., Demarty J., Cappelaere B., 2014. Long-term increase in diffuse groundwater recharge following expansion of rainfed cultivation in the Sahel, West Africa. Hydrogeology journal, 22: 1293-1305.

Issoufou H.B.A., Delzon S., Laurent J.P., Saâdou M., Mahamane A., Cappelaere B., Demarty J., Oï M., Rambal S., Seghieri S., 2013. Change in water loss regulation after canopy clear-cut of a dominant shrub in Sahelian agrosystems, Guiera senegalensis J.F. Gmel. Trees, 27: 1011-1022.

Issoufou H.B.A., Rambal S., Le Dantec V., Oï M., Laurent J.P., Saadou M., Seghieri J., 2015. Is the WBE model appropriate for semi-arid shrubs subjected to clear cutting? Tree physiology, 35: 197-208.

Jacobs C.M.J., 1994. Direct impact of atmospheric $\mathrm{CO}_{2}$ enrichment on regional transpiration. Ph.D. Thesis. Wageningen, Pays-Bas: Agricultural University, 179 p.

Kizito F., Dragila M., Sène M., 2006. Seasonal soil water variation and root patterns between two semi-arid shrubs co-existing with pearl millet in Senegal. West African journal of arid environment, 67: 436-455.

Lahmar R., Bationo B.A., Dan Lamso N., Guéro Y., Tittonell P., 2012. Tailoring conservation agriculture technologies to West Africa semi-arid zones: building on traditional local practices or soil restoration. Field crop research, 132: 158-167.

Louppe D., 1991. Guiera senegalensis : espèce agroforestière? Micro-jachère dérobée de saison sèche et approvisionnement énergétique d'un village du centre-nord du bassin arachidier sénégalais. Bois et forêts des tropiques, 228 : 41-47.

Lufafa A., Diédhiou I., Samba S.A.N., Séné M., Khouma M., Kizito F., Dick R.P., Dossa E., Noller J.S., 2008. Carbon stocks and patterns in native shrub communities of Senegal's peanut basin. Geoderma, 146: 75-8.

Lufafa A., Diédhiou I., Ndiaye N.A.S., Séné M., Kizito F., Dick R.P., Noller J.S., 2009. Allometric relationships and peak-season community biomass stocks of native shrubs in Senegal's peanut basin. Journal of arid environments, 73: 260-266.

Massuel S., Cappelaere B., Favreau G., Leduc C., Lebel T., Vischel T., 2011. Integrated surface water-groundwater modelling in the context of increasing water reserves of a regional Sahelian aquifer. Hydrological sciences journal, 56: 1242-1264. Doi:10.1080/02626667.2011.609171.

Parry M.L., Canziani O.F., Palutikof J.P., van der Linden P.J., Hanson C.E., (eds), 2007. Climate change 2007. Impacts, adaptation and vulnerability: contribution of working group II to the fourth assessment. Report of the intergovernmental panel on climate change. Cambridge, Royaume-Uni: Cambridge University Press.

Ramier D., Boulain N., Cappelaere B., Timouk F., Rabanit M., Lloyd C.R., Boubkraoui S., Metayer F., Descroix L., Wawrzyniak V., 2009. Towards an understanding of coupled physical and biological processes in the cultivated Sahel. 1. Energy and water. Journal of hydrology, 375: 204-216.

Saâdou M., 1990. La végétation des milieux drainés nigériens à l'est du fleuve Niger. Thèse de doctorat, Université de Niamey, Niger.

Safse, 2018. Recherche de compromis entre productions et services écosystémiques fournis par les systèmes agroforestiers tropicaux. https://safse.cirad.fr/ (consulté le 23 novembre 2018).

Velluet C., Demarty J., Cappelaere B., Braud I., Issoufou H.B.A., Boulain N., Ramier D., Mainassara G., Charvet G., Boucher M., Chazarin JP., Oï M., Yahou H., Maidaji B., Arpin-Pont F., Benarrosh N., Mahamane A., Nazoumou Y., Favreau G., Seghieri J., 2014. Building a field- and model-based climatology of local water and energy cycles in the cultivated Sahel - Annual budgets and seasonality. Hydrology and earth system sciences, 11: 4753-4808.

Wezel A., 2000. Scattered shrubs in pearl millet fields in semiarid Niger: effect on millet production. Agroforestry systems, 48: 219-228.

Wezel A., Rajot J.L., Herbrig C., 2000. Influence of shrubs on soil characteristics and their function in Sahelian agro-ecosystems in semi-arid Niger. Journal of arid environment, 44: 383-398. 


\section{Partie 3 \\ Exemples d'analyses transversales au regard de la diversité des systèmes agroforestiers tropicaux}





\title{
Chapitre 14 \\ Déterminants socio-économiques des dynamiques des systèmes agroforestiers
}

\author{
Pédelahore P., Droy I., Bidou J.-E., Freguin-Gresh S., \\ Sibelet N. et Le CoQ J.-F.
}

Résumé. Dans ce chapitre, sont comparés les déterminants des dynamiques spatiales et de recomposition de neuf systèmes agroforestiers situés au Bénin, au Cameroun, au Costa Rica, au Kenya et au Nicaragua. La comparaison s'appuie sur l'analyse de données collectées aux échelles de la parcelle, de l'exploitation agricole, de la zone d'étude et du pays. Une grille d'analyse permet d'identifier sept catégories de déterminants socio-économiques regroupées en quatre grandes classes : les stratégies des exploitants agricoles, la disponibilité en ressources, les caractéristiques des marchés, et les régulations et appuis en vigueur dans la zone étudiée. Les résultats obtenus montrent que les dynamiques spatiales (réduction, stabilité ou accroissement des surfaces des systèmes agroforestiers) sont déterminées par le niveau de disponibilité en terres et par les concurrences entre les différentes stratégies et les opportunités de valorisation monétaire de cette ressource offertes par le marché aux différents types d'acteurs économiques. La composition des systèmes agroforestiers (simples, moyennement complexes et complexes) est essentiellement déterminée par le type de producteurs et leurs stratégies. En règle générale, les grands producteurs disposant de hauts niveaux de capitaux privilégient des systèmes agroforestiers simples maximisant les revenus de la culture principale. Les petits producteurs développent généralement des systèmes agroforestiers complexes permettant une diversification des produits vivriers et marchands, ainsi qu'une réduction des risques alimentaires et commerciaux. L'évolution de la composition des systèmes agroforestiers (simplification, stabilité ou complexification) est déterminée par un changement des opportunités commerciales offertes par le marché et du degré de structuration et de sécurisation des filières concernées, par des incitations réglementaires ou liées à la labellisation des produits, ou par une modification des proportions relatives des différents types d'acteurs qui possèdent et gèrent ces systèmes agroforestiers (grands/petits producteurs, agriculteurs/agricultrices).

Abstract. This chapter compares the dynamics of nine agroforestry systems located in Benin, Cameroon, Costa Rica, Kenya and Nicaragua. It relies on the analysis of data 
collected at the plot, farm, landscape and country levels. It proposes an analytical grid based on seven categories of socioeconomic determinants grouped into four clusters: farmers' strategies, resource availability, market context, and current regulations and policy support in the study area. Results showed that spatial dynamics (reduction, stability or increase of the AFS area) were determined by land availability and competitions between the various strategies and opportunities for valuation of this resource by different stakeholders in relation to the market. The composition of the AFS (simple, moderately complex, complex) was essentially determined by the farmer category. In general, large farmers with high levels of capital developed simple AFS that maximized the income of the main crop. In general, small-scale farmers developed complex AFS that allowed diversification of food and cash crops and reduce food and commercial risks. The evolution of the composition of the SAF (simplification, stability or more complex) was determined either by a change in the opportunities offered by the market and the level of structuration of concerned value chains, or by regulatory or product labeling incentives, or by changes in the relative proportions of the different types of actors who own and manage these AFS (large / small farmers, man/women).

\section{W Expliciter les déterminants socio-économiques des dynamiques de transformations des systèmes agroforestiers}

Les systèmes agroforestiers sont présentés comme des systèmes capables de produire durablement des denrées agricoles et des produits forestiers ligneux ou non ligneux, et de fournir d'autres services écosystémiques ${ }^{1}$ (MEA, 2005) comme la préservation de la biodiversité ou de la fertilité des sols (Garrity et al., 2006; Rapidel et al., 2011). De nombreux programmes de recherche (Cafnet, 2007-2010, AFS4Food 20122015...) et de développement (Murray et Bannister, 2004) œuvrent pour améliorer les performances et le développement de ces systèmes. Le succès de ces projets est variable selon les sites et les contextes (Mercer et al., 2014). De nombreuses études ont cherché à expliquer les causes de cette variabilité et, en particulier, à en expliciter les déterminants socio-économiques (Sood et Mitchell, 2009). D’autres études décrivent les dynamiques de développement des systèmes agroforestiers existant dans le monde et en précisent les rythmes (différentes phases de transformation dans le temps) et les déterminants (Piketty et al., 2002). Ces études portent généralement sur un pays ou une zone particulière et mettent en évidence une grande diversité de déterminants, tant socio-économiques que biophysiques. Si quelques travaux ont souligné des récurrences entre différentes études portant sur l'identification des déterminants de l'adoption des systèmes agroforestiers par les exploitants agricoles dans le cadre de divers projets de développement (Pattanayak et al., 2003), peu de travaux ont comparé des dynamiques de développement des systèmes agroforestiers (Ruf, 1995).

Dans ce chapitre, nous saisissont l'occasion offerte par le projet de recherche Safse pour mener à bien une analyse comparée des dynamiques des systèmes agroforestiers

1. Les services écosystémiques sont définis comme étant les bénéfices que les êtres humains tirent du fonctionnement des écosystèmes. 
dans neuf terrains situés dans cinq pays africains et deux pays d'Amérique centrale (Bénin, Cameroun, Kenya, Nicaragua et Costa Rica). On observe des récurrences sur des différents terrains : mêmes dynamiques, mêmes déterminants socio-économiques de ces dynamiques. Tout d'abord, sont présentées les caractéristiques des terrains étudiés et la méthode utilisée pour décrire et représenter graphiquement les dynamiques étudiées et leurs déterminants socio-économiques. Puis, ces neuf situations sont analysées et comparées.

\section{W Des terrains contrastés et une méthodologie identique}

Les caractéristiques des neuf terrains étudiés dans 5 pays sont présentées dans le tableau 14.1. Elles mettent en évidence la diversité des contextes et des types de systèmes agroforestiers analysés.

\section{Carcatérisation des sites d'étude}

Lanalyse présentée porte sur deux dynamiques de transformation des systèmes agroforestiers :

- les dynamiques spatiales des systèmes agroforestiers, caractérisées par un indicateur unique qui concerne la réduction, le maintien ou l'accroissement des surfaces (en hectares) occupées par les divers types de systèmes agroforestiers étudiés;

- les dynamiques de recomposition des systèmes agroforestiers, caractérisées par deux indicateurs. Le premier est la composition ou nombre d'espèces présentes dans le système agroforestier au moment de l'étude. Le deuxième est l'évolution de cette composition au cours du temps.

Cette analyse vise également à identifier les principaux déterminants socio-économiques de ces deux dynamiques. Cette démarche analytique s'appuie sur la collecte et l'analyse de données recueillies à quatre niveaux :

- la parcelle. C'est à cette échelle que la composition du système agroforestier est caractérisée (nombre d'espèces présentes);

- l'exploitant agricole et son exploitation. Des entretiens semi-directifs conduits auprès d'un échantillon représentatif précisent leur dotation en facteurs de production (terre, travail, capital), leurs objectifs et leurs stratégies, ainsi que les déterminants de ces stratégies;

- la zone d'étude. Lanalyse des données cartographiques (cartes, photographies aériennes et par satellite) et statistiques existantes, ainsi que des entretiens auprès de personnes ressources (responsables des fédérations de groupement de producteurs, conseillers agricoles, autorités coutumières ou régionales, membres d'ONG...) précisent et quantifient les dynamiques spatiales et de recomposition des systèmes agroforestiers et leurs déterminants;

- le niveau national. À cette échelle, les données statistiques des principales productions issues des différents systèmes agroforestiers (café, cacao, karité...) sont souvent disponibles sur le long terme. Ces données sur les filières permettent de recouper, et parfois de préciser, les informations sur les dynamiques spatiales obtenues au niveau de la zone étudiée ou au niveau de l'exploitation. 


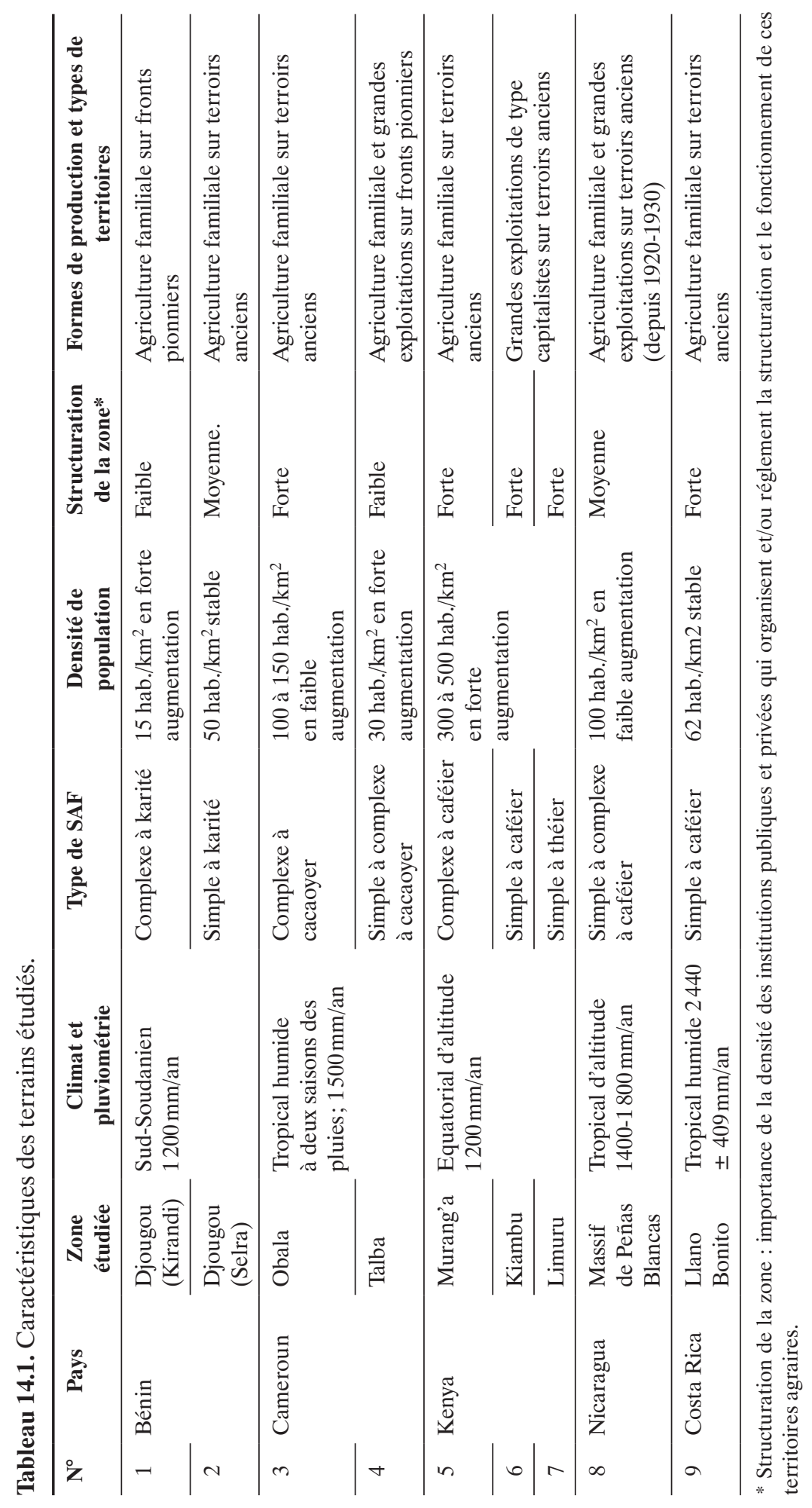




\section{Méthodes d'analyse}

La robustesse des informations collectées et des analyses réalisées s'appuie sur les méthodes de la triangulation, de l'itération et de la saturation (De Sardan, 1995).

Représentation graphique des résultats. La représentation graphique des résultats obtenus par cette démarche pour une situation donnée est faite à l'aide d'un graphique de type radar (fig. 14.1, 14.2, 14.3 et 14.4). Ce graphique représente les modalités des trois indicateurs mentionnés.

Le type de dynamique spatiale est figuré sur le graphique en radar par le triangle vert. Les modalités prises par cet indicateur sont numérotées de 1 à 5 selon la codification suivante :

- forte réduction des surfaces (1);

- réduction modérée de la surface (2);

- stabilité de la surface (3);

- augmentation modérée de la surface du système agroforestier (4);

- forte augmentation des surfaces (5).

Le type de composition des systèmes agroforestiers est figuré par le triangle orange. Les modalités prises par cet indicateur sont notées de 1 à 5 selon la codification suivante :

- monoculture (une espèce) (1);

- système agroforestier simplifié ( 2 à 3 espèces) (2);

- complexité moyenne (4 à 5 espèces) (3);

- complexe (6 à 10 espèces) (4);

- très complexe ( $>10$ espèces) (5).

Le type d'évolution de la composition du système agroforestier est figuré par une flèche incluse dans le triangle orange :

- une flèche dirigée vers le centre signifie que la dynamique évolue vers une simplification (diminution du nombre d'espèces) de la composition du système agroforestier;

- une flèche dirigée vers la périphérie du radar signifie que la dynamique devient plus complexe (accroissement du nombre d'espèces de la composition du système agroforestier);

- l'absence de flèche indique que la composition du système agroforestier est stable.

Les principaux déterminants socio-économiques de ces dynamiques sont répartis en quatre grandes classes ( I à Iv) qui regroupent sept catégories décrites dans le tableau 14.2.

Le type d'impact de chacun de ces déterminants est noté de 1 à 5 , suivant qu'il limite ou réduit (note 1 par exemple) la complexité de la composition (tracés oranges) ou les surfaces (tracés verts) des systèmes agroforestiers étudiés, ou au contraire qu'il accroit leur complexité et leur surface (note 5 par exemple). La note obtenue par le déterminant le plus limitant est la même que la note de la dynamique qu'il impacte.

Dans les différents graphiques, les déterminants socio-économiques sont classés par ordre croissant selon la note qu'ils obtiennent (1 à 5). Cela permet d'identifier le(s) déterminant(s) qui limite(nt) le plus la dynamique spatiale (augmentation des surfaces) ou la recomposition (vers plus de complexité) des systèmes agroforestiers étudiés. Leur ordre n'est donc pas le même selon le graphique considéré. Quand un déterminant n'a pas d'impact, il n'est pas noté. 
Tableau 14.2. Principaux déterminants socio-économiques des dynamiques spatiales et de recomposition.

\begin{tabular}{lll}
\hline \multicolumn{1}{c}{ Déterminant } & \multicolumn{1}{c}{ Catégorie } & \multicolumn{1}{c}{ Description } \\
\hline $\begin{array}{l}\text { I - Stratégies des } \\
\text { exploitants agricoles }\end{array}$ & $\begin{array}{l}\text { 1. Stratégies } \\
\text { développées }\end{array}$ & $\begin{array}{l}\text { Les stratégies développées par les différents types } \\
\text { d'exploitants agricoles (familiaux, entrepreneuriaux, } \\
\text { capitalistes...) sont définies par leurs objectifs et la façon } \\
\text { dont elles mobilisent leurs facteurs de production (terre, } \\
\end{array}$ \\
& & \\
& & \\
&
\end{tabular}

\begin{tabular}{|c|c|c|}
\hline \multirow[t]{3}{*}{$\begin{array}{l}\text { II - Disponibilité des } \\
\text { ressources }\end{array}$} & $\begin{array}{l}\text { 2. Disponibilité en } \\
\text { terre }\end{array}$ & $\begin{array}{l}\text { La disponibilité des ressources au niveau de l'ensemble de } \\
\text { la zone étudiée concerne les disponibilités en ressources } \\
\text { foncières (zone saturée ou front pionnier) }\end{array}$ \\
\hline & $\begin{array}{l}\text { 3. Disponibilité en } \\
\text { force de travail }\end{array}$ & $\begin{array}{l}\text { La disponibilité des ressources au niveau de l'ensemble } \\
\text { de la zone étudiée concerne les isponibilités en force de } \\
\text { travail (familiale, salariée...) }\end{array}$ \\
\hline & $\begin{array}{l}\text { 4. Disponibilité en } \\
\text { capital }\end{array}$ & $\begin{array}{l}\text { La disponibilité des ressources au niveau de l'ensemble de } \\
\text { la zone étudiée concerne les disponibilités en en capital } \\
\text { (physique, financier...) }\end{array}$ \\
\hline \multirow[t]{2}{*}{$\begin{array}{l}\text { III - Caractéristiques } \\
\text { des marchés de la zone } \\
\text { étudiée }\end{array}$} & 5. Marché prix & $\begin{array}{l}\text { Cette catégorie regroupe les déterminants liés aux } \\
\text { prix de vente des différents produits issus des systèmes } \\
\text { agroforestiers (produit principal comme le cacao ou } \\
\text { le café), ainsi que les productions associées (cultures } \\
\text { vivrières, fruits, bois...) }\end{array}$ \\
\hline & 6. Marché filière & $\begin{array}{l}\text { Cette catégorie regroupe les déterminants liés au niveau } \\
\text { d'organisation institutionnelle (relations contractuelles } \\
\text { ou non entre les différents acteurs de la filière) et } \\
\text { d'opérationnalité (réseaux routiers performants ou non, } \\
\text { structures de stockage présentes ou pas ...) des filières } \\
\text { concernées par ces systèmes agroforestiers }\end{array}$ \\
\hline IV - Régulations-appuis & $\begin{array}{l}7 . \\
\text { Régulations-appui }\end{array}$ & $\begin{array}{l}\text { Cette catégorie comprend les déterminants liés aux règles } \\
\text { et modalités de gouvernance des territoires (règles } \\
\text { coutumières d'accès au foncier, réforme foncière, } \\
\text { décentralisation...) et d'appui au secteur agricole (conseil } \\
\text { agricole, incitations financières pour la plantation ou la } \\
\text { conservation des arbres dans les systèmes agroforestiers ...) }\end{array}$ \\
\hline
\end{tabular}

\section{" Diversité et similarité des dynamiques systèmes agroforestiers et des déterminants observés}

Les figures 14 1, 14.2, 14.3 et 14.4 présentent les dynamiques des systèmes agroforestiers des sites étudiés et leurs déterminants.

\section{Analyse des systèmes agroforestiers au Bénin}

Les sites de Kirandi et Selra au Bénin (fig. 14.1) concernent des parcs à karité-néré. Ce système est marqué par des relations de genre au sein d'une agriculture familiale. Il est important de les prendre en compte pour comprendre son évolution : les hommes gèrent les arbres (sélection, protection ou coupe), les femmes exploitent les fruits. Leurs intérêts peuvent devenir divergents. 


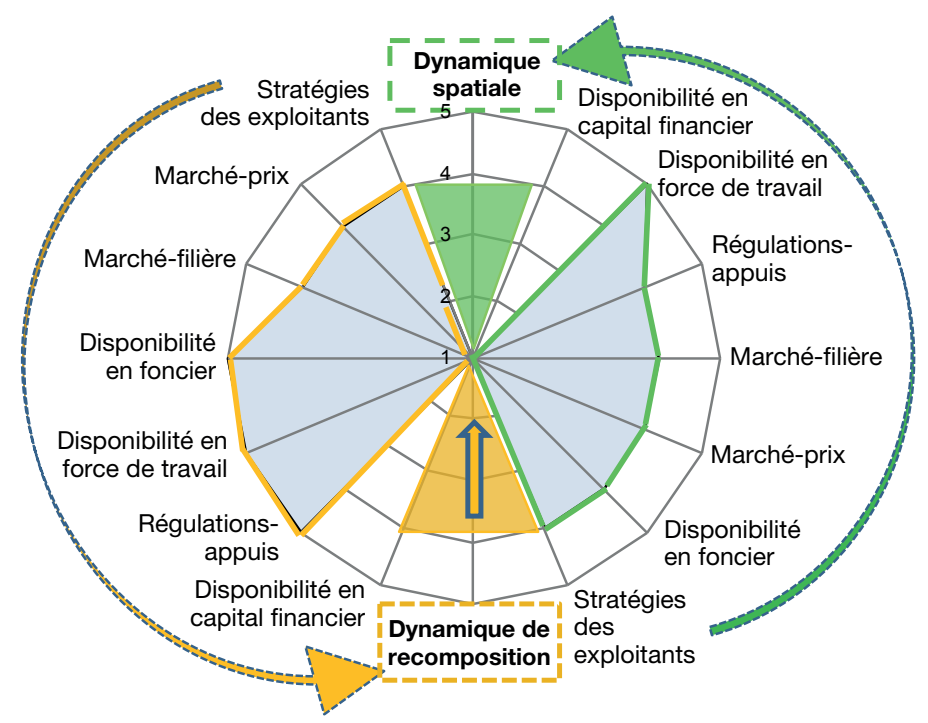

Figure 14.1a. Types de dynamiques des systèmes agroforestiers et déterminants au Bénin sur le site de Kirandi.

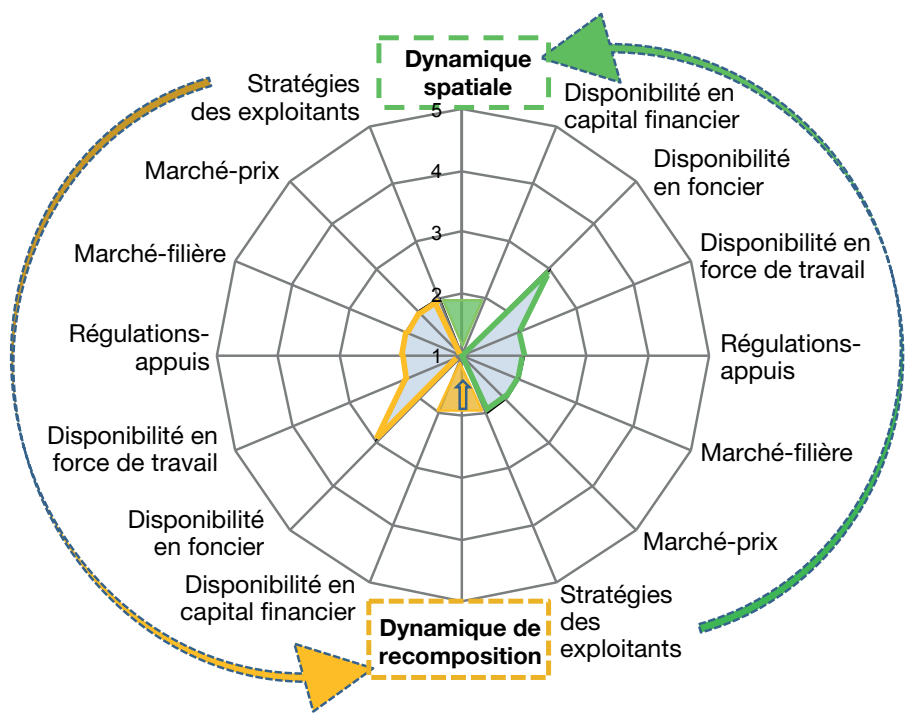

Figure 14.1b. Types de dynamiques des systèmes agroforestiers et déterminants au Bénin sur le site de Selra.

Dans les zones de front pionnier comme Kirandi, l'afflux de migrants à la recherche de nouvelles terres à exploiter et une forte disponibilité en foncier conduisent à une augmentation des surfaces de ces systèmes agroforestiers (note 4 , triangle vert). Ces migrants reproduisent le modèle de système agroforestier traditionnel à base de karité et de néré. Ce modèle répond à des besoins domestiques et à une économie locale marchande liée à ces deux filières. Bien que ces systèmes agroforestiers 
soient encore complexes (note 4, triangle orange, 6 à 10 espèces), le manque de préservation des jeunes plants de karité et de néré entraîne une simplification de la composition de ces systèmes agroforestiers (flèche vers le centre).

Dans les anciens terroirs de Selra, une double trajectoire de transformation des systèmes agroforestiers est à l'œuvre. Une partie des surfaces occupées par ces systèmes est remplacée (note 2, triangle vert) par des vergers d'anacardiers, des champs nus ou des systèmes agroforestiers qui voient la diversité de leur composition diminuer (flèche vers le centre). Cette diminution conduit à des systèmes simplifiés (note 2, triangle orange). Intéressés par les nouvelles opportunités économiques offertes par la filière anacardier, les détenteurs des droits sur la gestion des arbres sont les principaux responsables de ces transformations. À l'inverse de l'anacardier, le karité et le néré ne constituent pas un marqueur de propriété foncière (déterminant «régulation-appui», tab. 14.2). Ce fait accélère ces processus de disparition progressive du parc à karité-néré.

\section{Analyse des systèmes agroforestiers au Cameroun}

Les sites d'Obala et de Talba au Cameroun (fig. 14.2) concernent des systèmes agroforestiers à base de cacaoyers. Le site d'Obala correspond à une ancienne zone d'agriculture familiale. Les contraintes foncières expliquent que la surface de ces systèmes agroforestiers est aujourd'hui stabilisée (note 3, triangle vert). Les agriculteurs de ces zones développent des systèmes très complexes à base de cacaoyers (note 5, triangle orange) qui leur permettent de maintenir cette culture sous ombrage et de diversifier les productions (fruits, musacées...) pour satisfaire leurs besoins alimentaires et les différents marchés à l'export ou locaux, tout en limitant les risques.

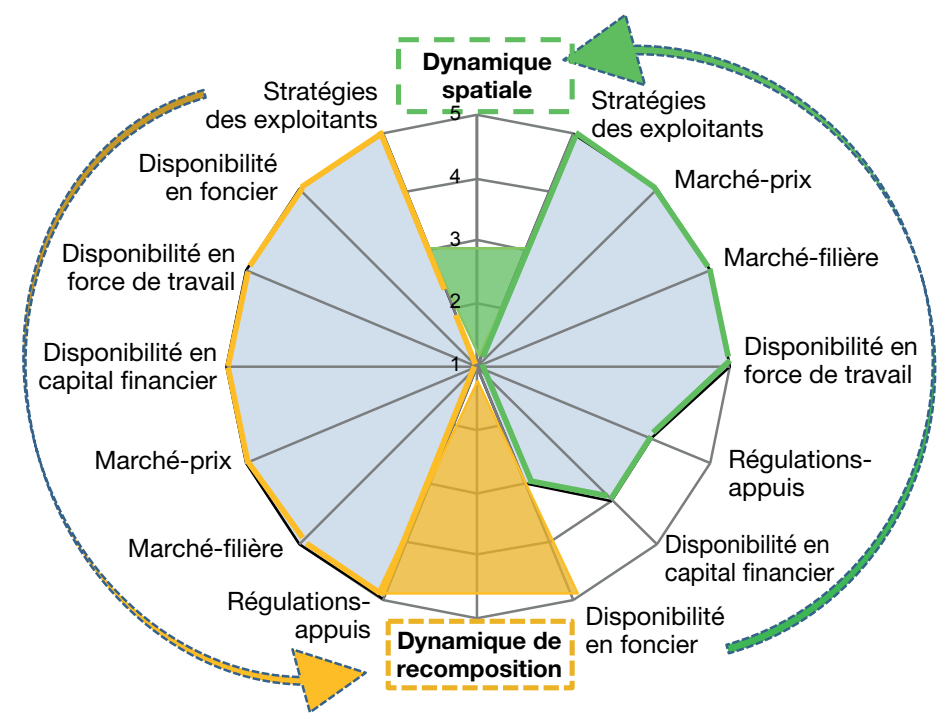

Figure 14.2a. Types de dynamiques des systèmes agroforestiers et déterminants au Cameroun sur le site d'Obala. 


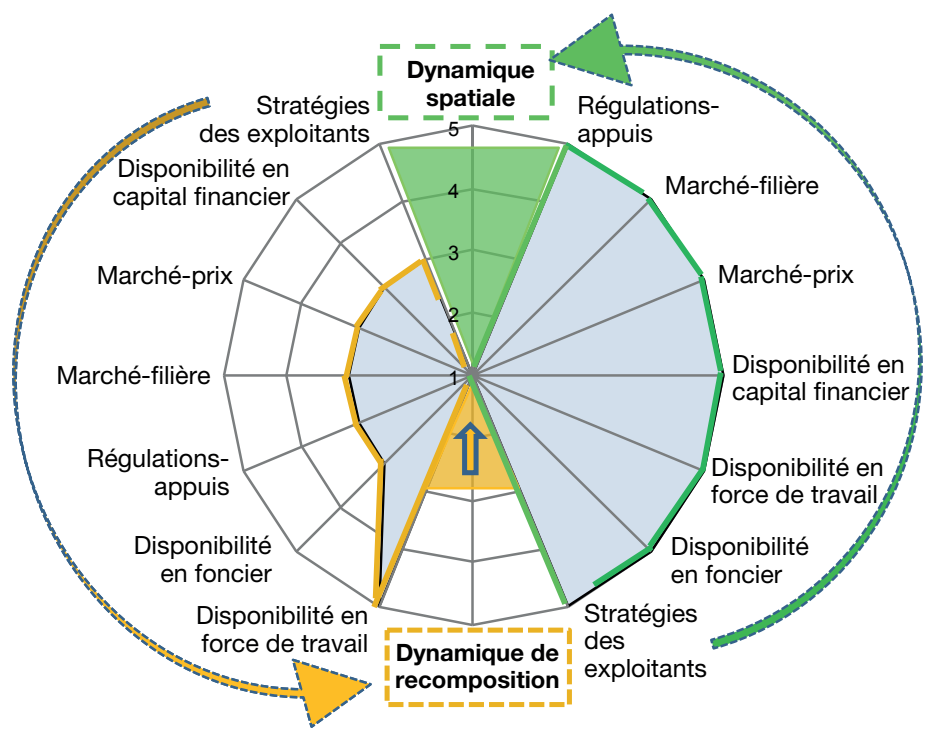

Figure 14.2b. Types de dynamiques des systèmes agroforestiers et déterminants au Cameroun sur le site de Talba.

Le site de Talba est une zone de front pionnier où les surfaces forestières sont rapidement remplacées par des systèmes agroforestiers à base de cacaoyers. Cette rapide expansion des surfaces (note 5 pour le triangle vert) est portée par des exploitants de type familiaux, patronaux ou capitalistes. Aujourd'hui, ces deux derniers types d'exploitants agricoles contrôlent $75 \%$ des surfaces plantées, ils simplifient des systèmes agroforestiers (note 3 pour le triangle orange et flèche vers le centre) pour maximiser les profits qu'ils tirent de la production cacaoyère.

\section{Analyse des systèmes agroforestiers au Kenya}

Les sites de Murang'a et de Kiambu au Kenya (fig. 14.3) concernent des systèmes agroforestiers à base de caféiers. Le site de Limuru concerne des systèmes agroforestiers à base de théiers.

Les systèmes agroforestiers à base de caféiers du site de Murang'a ont des surfaces stables (note 3 pour le triangle vert) du fait des fortes contraintes foncières de cette zone densément peuplée. Ces systèmes se sont progressivement complexifiés pour accroître la diversité des productions (bois de chauffage et d'œuvre, café, fruits, musacées, cultures vivrières, plantes fourragères, petits élevages et élevage bovin laitier) et améliorer la productivité de la terre. Cette intensification en travail et en capital a été la réponse apportée par ces petits agriculteurs familiaux à la réduction progressive des surfaces disponibles par famille, à la forte croissance de la demande urbaine en produits vivriers végétaux ou animaux et à la baisse des prix et du niveau de structuration de la filière café. Les politiques locales encouragent cette dynamique. 


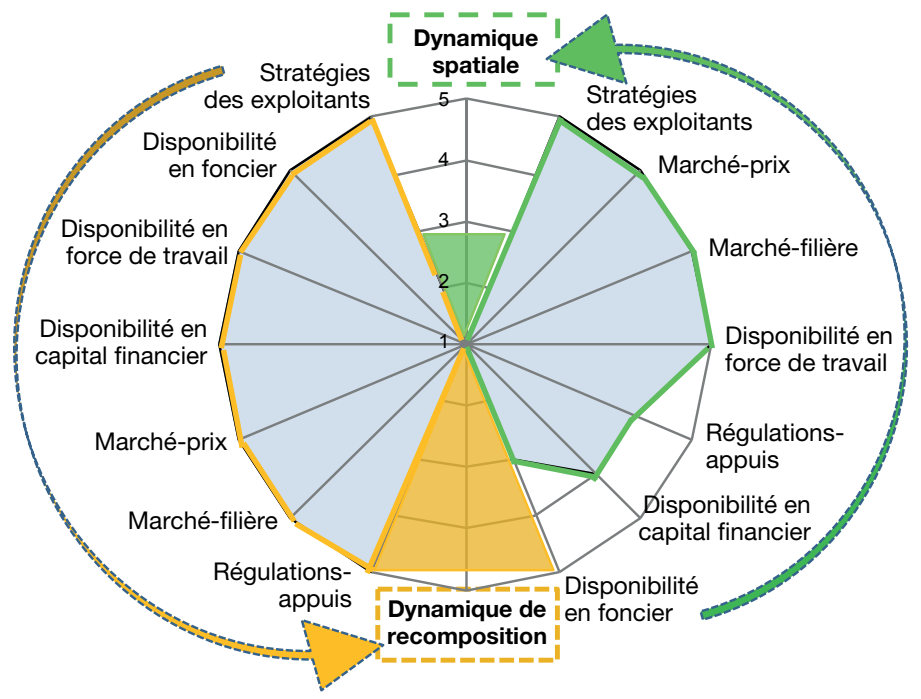

Figure 14.3a. Types de dynamiques systèmes agroforestiers et déterminants au Kenya sur le site de Murang'a, systèmes agroforestiers-caféiers.

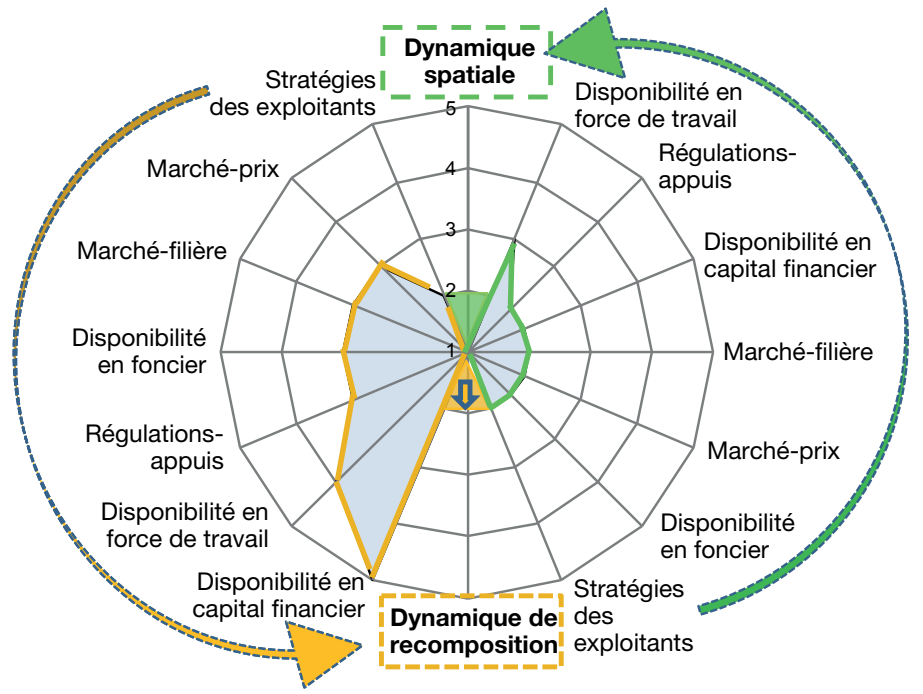

Figure 14.3b. Types de dynamiques systèmes agroforestiers et déterminants au Kenya sur les sites de Kiambu systèmes agroforestiers-caféiers et de Limuru systèmes agroforestiersthéiers.

Les deux sites de «Kiambu systèmes agroforestiers-caféiers» et de «Limuru systèmes agroforestiersthéier » ont été représentés par un seul graphique, car ils montrent les mêmes dynamiques et déterminants.

Les systèmes agroforestiers à base de caféiers ou de théiers des sites de Kiambu et de Limuru correspondent aux grandes exploitations patronales ou capitalistes qui produisent pour les marchés à l'export. La surface de ces systèmes est en baisse (note 2, triangle vert) parce que leurs propriétaires sont incités par les conditions actuelles de prix et de marché à les transformer en terrains immobiliers. Les 
dynamiques de complexification de ces systèmes agroforestiers (flèche vers l'extérieur), initialement quasi mono-spécifiques, correspondent à un accroissement de la présence et de la densité des arbres d'ombrage (note 2, triangle orange). Cette complexification est portée par le développement des filières de qualité qui se revendiquent du label écologique (Rainforest alliance, ...). Elle est également encouragée par les pouvoirs publics («régulation-appui»).

\section{Analyse des systèmes agroforestiers au Nicaragua et au Costa Rica}

Les sites de Peñas Blancas au Nicaragua et de Llano Bonito au Costa Rica (fig. 14.4) concernent des systèmes agroforestiers à base de caféiers. Le site de Peñas Blancas (Nicaragua) est caractérisé par une grande diversité de types d'exploitants agricoles, tant du point de vue des surfaces exploitées que des systèmes de production développés (céréales et légumineuses, systèmes agroforestiers caféiers, élevage...). Ce site est caractérisé par une stabilité des surfaces de systèmes agroforestiers à base de caféiers (note 3 pour le triangle vert). Cette stabilité s'explique par des contraintes foncières et de la concurrence pour l'espace exercée par les autres systèmes de production. Les systèmes agroforestiers des petits exploitants sont d'une complexité élevée et comportent des musacées, des arbres d'ombrage fournisseurs de bois de chauffage et d'œuvre, des arbres fruitiers et des arbres légumineux plantés à des fins agronomiques spécifiques (engrais vert, ombrage...). Bien que les pouvoirs publics et le développement des filières de qualité incitent à maintenir, voire à augmenter, la diversité et la densité des arbres dans les systèmes agroforestiers à base de caféiers, la montée en puissance de grands exploitants agricoles dans ce territoire conduit à leur simplification progressive (note 2 , triangle orange et flèche vers le centre).

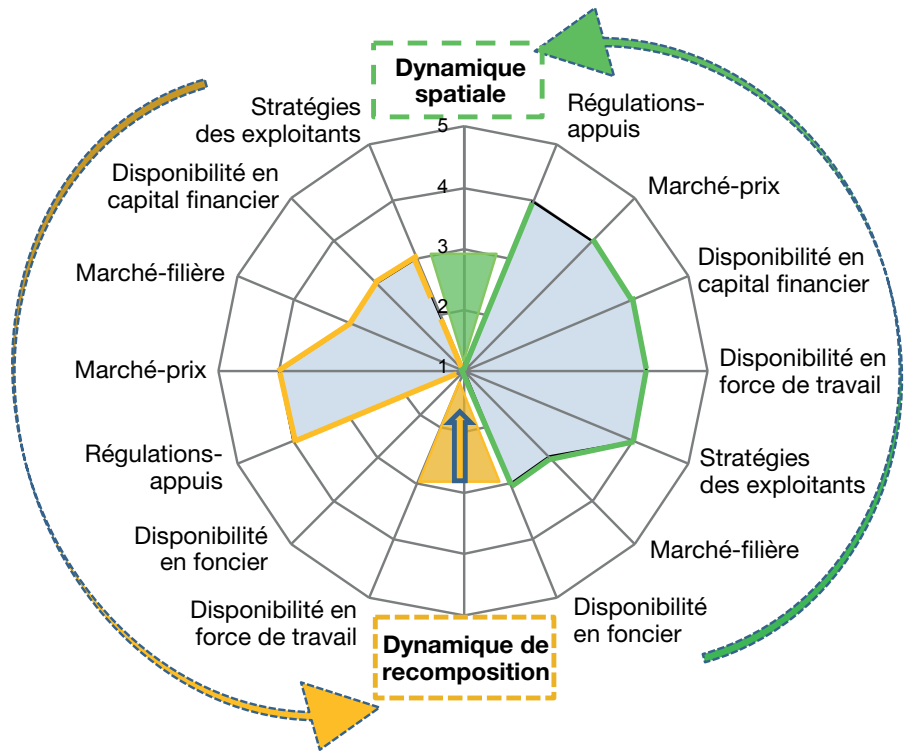

Figure 14.4a. Types de dynamiques systèmes agroforestiers et déterminants au Nicaragua sur le site de Peñas Blancas, Nicaragua. 


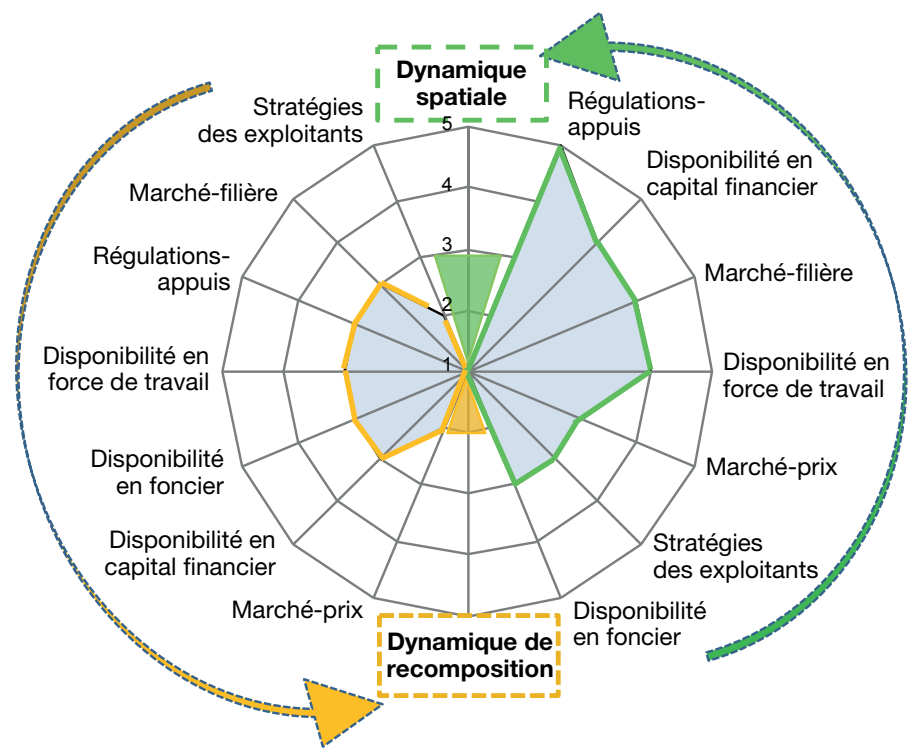

Figure 14.4b. Types de dynamiques systèmes agroforestiers et déterminants au Costa-Rica sur le site de Llano Bonito, Costa Rica.

Le site de Llano Bonito au Costa-Rica se caractérise par la stabilité en surface des systèmes agroforestiers à base de caféiers (note 3, triangle vert). Ces systèmes agroforestiers sont développés par des agriculteurs familiaux soumis à de fortes contraintes foncières; tout le territoire où la culture des caféiers est possible est cultivé. Les systèmes agroforestiers de Llano Bonito sont simples et homogènes (note 2 pour le triangle orange). Le caféier est cultivé sous ombrage de bananiers (Musa sp.) et d'une à deux espèces d'arbres (Erythrina sp.). La composition simple est maintenue par les petits producteurs. Cette stabilité s'explique par le fait que cette composition répond aux objectifs des agriculteurs en termes de revenus obtenus et de quantité de temps de travail qu'ils souhaitent investir. Et ce, même si leurs moyens de production (capital et travail), les conditions de marché (filières de café de qualité souvent reconnues par des certifications socio-environnementales) et les incitations des pouvoirs publics (protection des bassins versants du barrage hydroélectrique, protection des sources des cours d'eau) pourraient contribuer à une dynamique de complexification de ces systèmes agroforestiers. À Llano Bonito, comme dans la situation précédente du Nicaragua, l'intensification en capital et en travail des systèmes agroforestiers à base de caféiers passe par un renouvellement des variétés de caféier (plus résistantes aux maladies ou plus productives), un accroissement du recours aux intrants (engrais) et une meilleure maitrise des différentes opérations techniques (désherbage, taille des caféiers, gestion de l'ombrage...), et non par une complexification de leur composition. 


\section{- Enseignements de l'analyse des neuf sites sur les dynamiques et les déterminants des systèmes agroforestiers}

Le tableau 14.3 résume les caractéristiques des dynamiques spatiales et de recomposition à l'œuvre sur les neuf sites étudiés. Pour en simplifier la lecture et l'analyse, ce tableau a regroupé les cinq notes utilisées pour caractériser les dynamiques spatiales et la composition des systèmes agroforestiers dans les quatre figures précédentes en seulement trois catégories. La correspondance entre ces trois catégories et les cinq notes précédentes sont précisées dans ce même tableau (tab. 14.3).

Tableau 14.3. Nombre de sites par types de dynamique spatiale et de recomposition.

\begin{tabular}{|c|c|c|c|c|}
\hline \multirow{2}{*}{$\begin{array}{l}\text { Type de dynamique } \\
\begin{array}{l}\text { Dynamiques } \\
\text { spatiales }\end{array}\end{array}$} & \multirow{2}{*}{$\begin{array}{l}\begin{array}{c}\text { Indicateur de } \\
\text { recomposition }\end{array} \\
\text { Évolution } \\
\text { des surfaces }\end{array}$} & \multicolumn{3}{|c|}{ Modalité et nombre de sites } \\
\hline & & $\begin{array}{l}\text { Réduction des } \\
\text { surfaces (note } 1 \text { à } \\
2 \text {, triangle vert), } \\
3 \text { sites }\end{array}$ & $\begin{array}{l}\text { Stabilité des } \\
\text { surfaces (note } 3, \\
\text { triangle vert), } \\
4 \text { sites }\end{array}$ & $\begin{array}{l}\text { Augmentation } \\
\text { des surfaces } \\
\text { (note } 4 \text { à } 5, \\
\text { triangle vert), } \\
2 \text { sites }\end{array}$ \\
\hline \multirow[t]{2}{*}{$\begin{array}{l}\text { Dynamiques de } \\
\text { recomposition }\end{array}$} & Composition & $\begin{array}{l}\text { Simples (note } 1 \\
\text { à } 2 \text {, triangle } \\
\text { orange), } 5 \text { sites }\end{array}$ & $\begin{array}{l}\text { Moyennement } \\
\text { complexes } \\
\text { (note } 3 \text { pour le } \\
\text { triangle orange), } \\
1 \text { site }\end{array}$ & $\begin{array}{l}\text { Complexes } \\
\text { (note } 4 \text { à } 5, \\
\text { triangle orange), } \\
3 \text { sites }\end{array}$ \\
\hline & $\begin{array}{l}\text { Évolution } \\
\text { de la composition }\end{array}$ & $\begin{array}{l}\text { Simplification } \\
\text { (flèche vers le } \\
\text { centre), } 4 \text { sites }\end{array}$ & $\begin{array}{l}\text { Stabilité (pas de } \\
\text { flèche), } 2 \text { sites }\end{array}$ & $\begin{array}{l}\text { Complexification } \\
\text { (flèche vers la } \\
\text { périphérie), } \\
3 \text { sites }\end{array}$ \\
\hline
\end{tabular}

Ce tableau montre d'abord que les neuf sites étudiés ont permis de couvrir toutes des modalités pour chacun des trois indicateurs retenus pour caractériser les dynamiques à l'œuvre.

Pour chacun des trois indicateurs retenus, l'impact des différents déterminants socio-économiques est analysé.

\section{Évolution de la surface des systèmes agroforestiers}

Dans quatre sites, la surface est stable : Obala (Cameroun), Murang'a (Kenya), Peñas Blancas (Nicaragua) et Llano Bonito (Costa Rica). Cette stabilité est liée aux contraintes foncières (saturation de l'espace agricole) qui empêchent leur extension. La stabilité globale au niveau d'un territoire donné peut cependant masquer des phénomènes de compensation, comme dans le cas de Peñas Blancas où l'accroissement des surfaces de systèmes agroforestiers-caféiers porté par les grands exploitants compense la réduction des surfaces systèmes agroforestiers-caféiers conduite par les petits exploitants agricoles (remplacement des systèmes agroforestiers-caféiers par des céréales et des légumineuses). Cette stabilité peut aussi s'expliquer par l'action 
des pouvoirs publics qui freinent considérablement, voire empêchent, cette extension du fait du classement en réserve naturelle des terres bordant ces territoires agraires.

Laugmentation des surfaces de systèmes agroforestiers concerne deux sites : les fronts pionniers de Talba (Cameroun) et Kirandi (Bénin). Elle dépend des disponibilités foncières et de l'existence de différents types d'exploitants agricoles (familiaux, patronaux et capitalistes) en capacité (disposant de force de travail ou de capitaux) et désireux, du fait des conditions de marché, de les mettre en valeur. Ces observations rejoignent des travaux antérieurs ayant mis en évidence l'importance des disponibilités foncières et en force de travail comme moteur de ces dynamiques d'extension (Ruf, 1995). Le contexte réglementaire et les incitations ou appui de la puissance publique, s'ils existent et s'ils sont appliqués, vont freiner ou accélérer ces dynamiques d'extension portées par les stratégies des exploitants agricoles et les conditions de marché.

Dans trois des sites étudiés, la surface des systèmes agroforestiers est en réduction : Selra (Bénin), Kiambu et Limuru (Kenya). En premier lieu, c'est l'évolution des conditions de marché qui détermine cette réduction des surfaces. Ces sites se caractérisent par de nouveaux rapports de prix entre les différentes productions issues des systèmes agroforestiers ou entre les productions issues des systèmes agroforestiers et d'autres usages possibles du sol. Les exploitants vont s'adapter à ces changements de l'environnement économique. Dans le cas de Selra, l'émergence d'une filière anacardier, mieux organisée et plus rémunératrice que la production de karité et de néré, conduit à la disparition d'une partie des surfaces de systèmes agroforestiers à base de karité.

Ces transformations traduisent également l'évolution des rapports de force dans un territoire donné (Pélissier, 1980). Dans le cas de Selra, ces transformations traduisent l'éviction des femmes, traditionnellement collectrices et transformatrices du karité et du néré, au profit des hommes détenteurs du foncier et des plantations d'anacardier. Dans le cas des systèmes agroforestiers-caféiers de Kiambu et des systèmes agroforestiers-théiers de Limuru, c'est l'émergence d'un marché immobilier, bien plus rémunérateur que la production agricole, qui entraîne la disparition des systèmes agroforestiers. Ce type de transformation n'est pas propre au Kenya. Des évolutions comparables sont observées par exemple en périphérie de San José au Costa Rica où les systèmes agroforestiers sont remplacés par des espaces urbains. Ce type d'évolution est peu cité dans la littérature et nos travaux témoignent de l'accroissement de la concurrence entre espace rural et espace urbain et de leurs impacts sur la réduction des surfaces de systèmes agroforestiers.

\section{Évolution de la composition des systèmes agroforestiers}

Lanalyse porte sur la composition en nombre d'espèces des systèmes agroforestiers. Les déterminants du niveau de complexité des systèmes agroforestiers relèvent principalement des stratégies des exploitants agricoles, de leur accès aux facteurs de production et de l'évolution des marchés (prix, organisation des filières). Au Kenya (Murang'a) et au Cameroun (Obala), les petits producteurs familiaux développent des systèmes agroforestiers complexes pour accroître la productivité de la terre et du travail, diversifier les productions et minimiser les risques. Quant aux grands exploitants de type patronal ou capitaliste (Talba, Kiambu et Limuru au Kenya), 
ils développent des systèmes agroforestiers simples permettant de rendre plus efficiente la gestion de la force de travail ouvrière et de maximiser les profits issus de la culture principale. Toutefois, les sites de Selra (Bénin), de Peñas Blancas (Nicaragua) et de Llano Bonito (Costa-Rica) montrent que les petits agriculteurs familiaux peuvent également développer des systèmes agroforestiers simples ou moyennement complexes pour leur adaptation aux conditions pédoclimatiques du lieu, et à leurs propres objectifs en termes de niveau de revenu et d'investissement en travail, et aux conditions de marché. Les petits producteurs simplifient la composition de leurs systèmes agroforestiers lorsque le prix de vente d'un des produits, qui en est issu, est particulièrement élevé par rapport aux autres et que la filière du produit concerné (café en Amérique cntrale, anacarde au Bénin) est bien structurée et sécurise la vente du produit. Les travaux conduits sur les systèmes agroforestiers-caféiers par Soini (2005) en pays Shaga montrent que le même type de réponse paysanne a accompagné le renforcement de la structuration de la filière café en Tanzanie. Ainsi, la composition des systèmes agroforestiers et leur évolution dépendent aujourd'hui en grande partie des pratiques alimentaires et techniques locales, et surtout des stratégies d'adaptation des exploitants agricoles aux tendances du marché et au niveau de structuration des filières agricoles.

\section{Évolution de la composition des systèmes agroforestiers}

La composition des systèmes agroforestiers reste stable sur deux sites (Obala et Llano Bonito), se simplifie sur quatre sites (Selra, Kirandi, Talba, Peñas Blancas) et se complexifie sur trois sites (Murang'a, Kiambu et Limuru). La stabilité de la composition traduit la bonne adéquation de cette composition avec les objectifs, les pratiques alimentaires, les techniques locales et les stratégies de l'exploitant, ainsi que la stabilité du marché ou du contexte règlementaire. À l'inverse, les évolutions de la composition des systèmes agroforestiers (simplification ou complexification) traduisent les adaptations des différents types d'exploitants agricoles aux changements des conditions de production, de marché ou règlementaires. Elles montrent également la transformation des rapports de force entre les différents types d'exploitants agricoles (homme/femme, petits/grands exploitants...) et leur importance relative sur le territoire étudié.

Ces deux types de dynamiques, spatiale et de recomposition des systèmes agroforestiers, peuvent avoir des liens entre elles. En situation de forte contrainte foncière et de stabilité des surfaces de systèmes agroforestiers par exemple, les agriculteurs peuvent être amenés à accroître la complexité des systèmes agroforestiers pour augmenter la productivité à l'hectare (par exemple à Murang'a au Kenya).

\section{- Robustesse et extrapolation de la méthodologie}

La grille d'analyse proposée est utile pour comparer les dynamiques dominantes de sites très divers et en identifier les principaux déterminants socio-économiques.

Robustesse. La grille d'analyse proposée pour identifier et pondérer les déterminants socio-économiques des dynamiques spatiales et de recomposition des systèmes 
agroforestiers est robuste. Elle permet de comparer des sites qui présentent des caractéristiques et des trajectoires de systèmes agroforestiers contrastées. Cependant, les variables choisies pour caractériser ces dynamiques simplifient la réalité.

En effet, à partir de cette grille, les dynamiques spatiales dominantes sont décrites par les variations de surface de systèmes agroforestiers pour l'ensemble des territoires étudiés. Elles peuvent masquer des évolutions différentes entre les divers types d'exploitantions agricoles ou de sous-zones. De même, la variable «composition» ne décrit la composition et la structure des systèmes agroforestiers, ainsi que leurs évolutions qu'à travers le nombre d'espèces différentes en présence. Elle masque d'éventuels changements de système de culture à nombre d'espèces égal comme la densité d'arbres et le niveau d'ombrage, ou les changements de variétés ou de pratiques techniques.

Cette grille d'analyse permet de construire des représentations intermédiaires utiles pour structurer et approfondir la production de connaissances sur les dynamiques des systèmes agroforestiers et leurs déterminants. Elle permet aussi d'organiser des échanges interdisciplinaires entre chercheurs, et entre les chercheurs et les acteurs locaux.

Similarités dans les trajectoires de transformation et les déterminants identifiés. Cette étude montre également que même si la diversité des dynamiques et des déterminants observés est importante, on retrouve des similarités, voire des récurrences, dans les trajectoires de transformation et les déterminants identifiés, ainsi que dans l'importance relative de ces différents déterminants. Les principales conclusions de nos observations sont les suivantes :

- les surfaces des systèmes agroforestiers restent stables dans le cas où les contraintes foncières sont importantes et les systèmes agroforestiers en place sont en cohérence avec les incitations des marchés. Elles augmentent lorsqu'il y a des disponibilités foncières et que le marché incite les producteurs déjà en place à accroître leurs surfaces, ou de nouveaux producteurs ou investisseurs à les développer. Elles régressent lorsque d'autres opportunités de valorisation du foncier mieux rémunérées (immobilier, autres productions marchandes) se développent au détriment des systèmes agroforestiers existants;

- la composition (multiespèces) des systèmes agroforestiers reste stable si elle est en cohérence avec les habitudes alimentaires, les techniques et les stratégies commerciales des exploitants. La composition se simplifie lorsqu'il y a une modification des types de producteurs qui investissent ces territoires agraires (grands producteurs/ petits producteurs, hommes/femmes). La composition se simplifie également par le fait des petits producteurs lorsqu'une production issue du système agroforestier est bien mieux rémunérée que les autres et que la filière concernée est fortement structurée et garantit des débouchés commerciaux;

- la composition se complexifie lorsque les petits producteurs sont à la recherche de diversification de leurs produits et de stratégies anti-risques. C'est aussi le cas lorsque les gros producteurs sont incités par des mesures règlementaires ou des labels à introduire des arbres d'ombrage, voire d'autres espèces, dans des plantations d'abord quasi mono-spécifiques.

Ces premiers enseignements méthodologiques et sur les dynamiques des systèmes agroforestiers et leurs déterminants socio-économiques pourront être complétés par 
la poursuite d'études sur des terrains variés et la mise en débat des résultats obtenus, tant avec des chercheurs qu'avec les acteurs locaux. C'est la mise à l'épreuve répétée de ce cadre d'analyse dans des situations contrastées et un travail de réflexivité critique collectif qui pourront permettre d'en améliorer progressivement la portée euristique et opérationnelle.

\section{$\rightarrow$ Bibliographie}

Garrity D.P., Okono A., Grayson M., Parrott S., 2006. World Agroforestry into the Future. Nairobi: World agroforestry center. http://www.worldagroforestry.org/downloads/Publications/PDFS/b14409.pdf.

MEA, 2005. Ecosystems and human well-being: Synthesis. Washington, DC : Island Press.

Mercer D.E., Frey G.E., Cubbage F.W., 2014. Economics of agroforestry. In: Handbook of Forests Economics. Kant S., Alavalapati J.R.R., (eds). New York: Earthscan from Routledge.

Murray G.F., Bannister M.E., 2004. Peasants, agroforesters, and anthropologists: A 20-year venture in income-generating trees and hedgerows in Haiti. Agroforestry systems, 61: 383-397.

De Sardan J.P., 1995. La politique du terrain. Sur la production des données en anthropologie. Enquête, 1 : 71-109.

Pattanayak S.K., Mercer D.E., Sills E., Yang J.C., 2003. Taking stock of agroforestry adoption studies. Agroforestry systems, 57: 173-186.

Pélissier P., 1980. Larbre dans les paysages agraires de l'Afrique noire. Cahier Orstom, série Sciences humaines, 17(3-4) : 131-136.

Piketty M.G., Veiga J.B., Poccard-Chapuis R., Tourrand J.F., 2002. Le potentiel des systèmes agroforestiers sur les fronts pionniers d'Amazonie brésilienne. Bois et forêts des tropiques, 2(272) : 75-87.

Rapidel B., Le Coq J.F., DeClerck F., Beer J., 2011. 'Measurement and payment of ecosystem services from agriculture and agroforestry. New insights from the neotropics. In: Ecosystem services from agriculture and agroforestry: measurement and payment. Le Coq J.F., Rapidel B., (eds). Londres, Royaume-Uni : Earthscan publications.

Ruf F., 1995. Booms et crises du cacao. Les vertiges de l'or brun. Paris: Karthala, Cirad, ministère de la Coopération.

Soini E., 2005. Changing livelihoods on the slopes of Mount Kilimanjaro, Tanzania: Challenges and opportunities in the Chaga home garden system. Agroforestry systems, 64(2): 157-167.

Sood K.K., Mitchell C.P., 2009. Identifying important biophysical and social determinants of on-farm tree growing in subsistence-based traditional agroforestry systems. Agroforestry systems, (75): 175-187. 



\title{
Chapitre 15 \\ Régulation des bioagresseurs des cultures dans les systèmes agroforestiers tropicaux, revue des approches
}

\author{
Bagny Beilhe L., Allinne C., Avelino J., Babin R., Brévault T., \\ Gidoin C., Ngo Bieng M.A., Motisi N., Soti V. et Ten Hoopen G.M.
}

Résumé. Au sein des systèmes agroforestiers tropicaux, de nombreuses interactions se déroulent dans et entre les environnements biotiques et abiotiques. Elles favorisent une régulation naturelle des bioagresseurs des cultures de ces systèmes. Afin d'exploiter au mieux cette régulation naturelle et de limiter les pertes de production, il est primordial de bien comprendre ces interactions. Ce chapitre présente une synthèse d'études de ces mécanismes de régulation, à partir de données empiriques sur des maladies et ravageurs dans des systèmes agroforestiers à base de caféiers, de cacaoyers et de mil au Cameroun, au Costa Rica, au Kenya et au Sénégal. En fonction des caractéristiques biologiques des bioagresseurs et de l'environnement dans lequel ils se développent, des approches multi échelles, de l'arbre au paysage, adaptées aux modèles étudiés ont été utilisées pour évaluer les stratégies de régulation ascendante par les ressources «bottom-up» et descendante par les ennemis naturels «top-down». Les approches développées ont permis d'évaluer l'effet de la composition et de l'organisation spatiale de la biodiversité associée au sein des systèmes agroforestiers sur les bioagresseurs, l'effet de l'ombrage sur le développement des bioagresseurs et l'effet de la biodiversité végétale associée aux échelles parcelle et paysage sur les communautés d'ennemis naturels et leur efficacité à réguler les bioagresseurs. Des approches expérimentales et intégratives, d'écologie des communautés et du paysage fondées notamment sur l'étude des traits fonctionnels se sont avérées nécessaires pour estimer au mieux les services de régulation.

Abstract. Tropical agroforestry systems are home to complex interactions between and within the biotic and abiotic environments, which govern natural regulation processes of pests and diseases of agricultural crops. In order to optimally exploit these control mechanisms, thereby limiting production losses, it is necessary to improve our understanding 
of these interactions within agro-ecosystems. This chapter presents an overview of several studies that looked at naturally occurring control mechanisms in tropical agroforestry systems. This synthesis has been elaborated based on empirical data from studies on the regulation of pests and diseases in coffee, cacao, and millet-based agroforestry systems in Cameroon, Costa Rica, Kenya and Senegal. Based on the biological characteristics of the pest and/or disease and the environment in which they develop, scale dependent approaches, from tree to landscape, appropriate to the models being studied, have been used to evaluate both "bottom-up" and "top-down" control mechanisms. The developed approaches allowed to evaluate: the effects of the composition and spatial organization of associated plant diversity on the regulation of pests and diseases; the effects of shade on the development of pests and diseases and the effects of associated plant biodiversity at plot and landscape level on communities of natural enemies and their efficacy in controlling pests and diseases. Experimental and integrative approaches from population and landscape ecology, taking into account functional traits, are necessary tools to understand regulation services.

\section{Introduction}

Dans les systèmes agroforestiers, les services de régulation des bioagresseurs résultent d'un ensemble de processus complexes qui interagissent entre eux à différentes échelles. Leffet net de la diversité végétale des agroécosystèmes sur les bioagresseurs varie en fonction des espèces cultivées ou non cultivées, des traits de vie de ces bioagresseurs, du profil des communautés d'ennemis naturels et des conditions du milieu (Malézieux, 2012; Ratnadass et al., 2012).

\section{Encadré 16.1 - Bottom-up control et Top-down control}

Dans le contrôle bottom up, l'abondance d'une population est régulée par les ressources trophiques. La production primaire conditionne les réseaux trophiques supérieurs via la compétition entre individus d'un même niveau trophique pour l'accès aux ressources.

Dans le contrôle top down, l'abondance d'une population est régulée par les prédateurs. La prédation détermine la répartition de la biomasse dans les niveaux trophiques inférieurs.

La composition et l'organisation spatiale de la biodiversité présente dans les systèmes agroforestiers, à l'échelle de la parcelle ou à celle du paysage peuvent avoir des effets directs ou indirects sur les bioagresseurs. Elles peuvent ainsi modifier le développement, la reproduction et les comportements de recherche de la ressource des bioagresseurs (i.e., bottom-up control) ou augmenter l'abondance de leurs ennemis naturels (i.e., top-down control). La dynamique des populations de bioagresseurs résulte en effet d'interactions dynamiques avec l'environnement biotique et abiotique, qui se produisent au cours du temps et dans l'espace au sein de la parcelle et du paysage (Gaba et al., 2015).

Dans le cas des stratégies bottom-up, la biodiversité associée aux cultures au sein des systèmes agroforestiers perturbe le repérage et la colonisation de l'hôte par le bioagresseur par un effet de dilution de la ressource. Elle réduirait aussi l'acces- 
sibilité du bioagresseur à la ressource en favorisant la fragmentation des habitats. Dans les systèmes agroforestiers, la diversité végétale associée favorise également l'ombrage (au sens d'altérations microclimatiques) dans la parcelle (Beer et al., 1997). Lombrage est nettement perceptible dans ces systèmes où chaque strate agit comme un filtre sur les variables microclimatiques. Cet ombrage peut avoir un effet direct sur le développement du bioagresseur ou un effet indirect par le biais de son action sur la plante hôte. En effet, la réduction de la quantité de tissus sensibles aux bioagresseurs par l'augmentation de l'ombrage serait un des principaux mécanismes de réduction de la sensibilité des caféiers et des cacaoyers, respectivement à la rouille des feuilles et aux mirides ou à la maladie du balai de sorcière (Avelino et al., 2011). La gestion de l'ombrage tient ainsi une place importante dans la gestion des bioagresseurs dans les systèmes agroforestiers.

Dans le cas des stratégies top-down, la biodiversité végétale influence indirectement les populations de bioagresseurs en favorisant le développement des ennemis naturels, à l'échelle de la parcelle ou du paysage (en le considérant comme une mosaïque d'habitats). Cette biodiversité végétale détermine l'abondance, la diversité et l'efficacité des communautés d'ennemis naturels via la fourniture de ressources (refuge, hôtes ou proies alternatifs, nourriture, etc.). En règle générale, la régulation écologique des bioagresseurs augmente avec la complexité du paysage (Evans, 2016). La composition et la configuration du paysage déterminent la disponibilité des ressources et leur accessibilité, par exemple par la présence de corridors biologiques ou au contraire de barrières physiques ou chimiques. Ces propriétés du paysage déterminent aussi la variabilité spatio-temporelle des populations de bioagresseurs et peuvent limiter ou retarder la colonisation des cultures.

\section{Encadré 16.2 - Définition de « régulation naturelle des bioagresseurs}

La régulation naturelle des bioagresseurs résulte des processus assurés par la biodiversité pour contrôler les populations de bioagresseurs en diminuant le nombre d'individus et/ou leur impact sur les cultures.

Dans le cadre du projet Safse, les stratégies bottom-up et top down de régulation des bioagresseurs dans les systèmes agroforestiers tropicaux ont été étudiées à différentes échelles (arbre, parcelle et paysage) (fig. 15.1). Plusieurs études de cas de systèmes agroforestiers tropicaux ont permis d'explorer les mécanismes sous-jacents à la régulation des bioagresseurs. Il s'agit des systèmes suivants :

- à base de caféiers au Costa Rica, au Cameroun et au Kenya;

- à base de cacaoyers au Cameroun dans des zones humides;

- à base d'acacias dans les zones semi-arides au Sénégal.

Les mécanismes étudiés ont été entre autres :

- l'effet de la composition et de l'organisation spatiale de la biodiversité au sein des systèmes agroforestiers sur les bioagresseurs (voir chapitre 7, Ngo Bieng et al.); - l'effet de l'ombrage sur le développement des bioagresseurs;

- l'effet de la biodiversité végétale associée sur les communautés d'ennemis naturels et leur efficacité à réguler les bioagresseurs aux échelles de la parcelle et du paysage. 


\section{" Régulation bottom-up des bioagresseurs par la biodiversité végétale associée dans les systèmes agroforestiers}

\section{Effet de l'organisation spatiale de la biodiversité associée sur les bioagresseurs}

Ngo Bieng et al. (chapitre 7) ont montré que l'organisation spatiale de la biodiversité végétale associée aux systèmes agroforestiers à base de cacaoyers permettait de réguler les bioagresseurs via des mécanismes complexes incluant la disponibilité de la ressource, l'effet de l'ombrage et les effets barrières physiques. L'étude des effets indépendants des variables de structure horizontale et verticale des arbres d'ombrage associés aux cacaoyers sur l'intensité de l'infestation permet une meilleure compréhension de l'effet de l'ombrage sur le cycle de vie de la maladie (Gidoin et al., 2013). Les effets de l'organisation spatiale des arbres d'ombrage sur la régulation des bioagresseurs ne seront pas traités spécifiquement dans ce chapitre de synthèse.

\section{Effet de l'ombrage sur le développement des bioagresseurs}

Les bioagresseurs sont plus ou moins sensibles aux variations du microclimat, en fonction de leurs caractéristiques de développement et de dispersion (Schroth et al., 2000). Leurs réponses aux altérations microclimatiques induites par la diversité végétale associée ne sont donc pas forcément les mêmes. Une augmentation de l'intensité de l'ombrage diminue la température moyenne et augmente l'humidité relative moyenne de la parcelle (Avelino et al., 2011). Ces modifications du microclimat peuvent affecter la dynamique des populations de bioagresseurs, leur incidence et leur action sur la culture. L'ombrage stimule de multiples mécanismes écologiques, en interaction avec le climat, dont les effets peuvent être antagonistes (négatifs, mais aussi positifs) sur les dynamiques des bioagresseurs (Ratnadass et al., 2012).

Dans le cas des maladies fongiques, on peut en général distinguer deux phases principales dans le cycle de vie de l'agent pathogène : la dispersion des spores et leur germination. Généralement, la germination des spores dépend du microclimat, elle est favorisée par des conditions de forte humidité relative et donc par l'ombrage (Schroth et al., 2000). La phase de dispersion du pathogène, quant à elle, dépend du microclimat et de son mode de dispersion. Par exemple, Moniliophthora roreri, l'agent pathogène de la moniliose du cacaoyer, se disperse par le vent. Si les conditions d'ombrage favorisent la germination, elles réduisent en revanche les mouvements d'air et augmentent l'humidité relative; ce qui a pour conséquence d'augmenter le poids des spores et de défavoriser la dispersion de $M$. roreri. Il est donc difficile, dans ce cas, de statuer sur un effet positif ou négatif de l'ombrage sur l'intensité de la maladie. Il n'est pas rare que les arbres d'ombrage limitent la dispersion des agents pathogènes en modifiant physiquement l'environnement. Par exemple, la réduction de l'impact des gouttes de pluie peut réduire la dispersion des spores par réduction des éclaboussures (Avelino et al., 2011). Dans le cas des ravageurs des cultures, il est également difficile d'établir des généralités, sachant que l'effet du microclimat 
est dépendant des caractéristiques des ravageurs (Schroth et al., 2000). Les arbres d'ombrage peuvent aussi représenter des barrières physiques au déplacement des insectes ravageurs.

Les effets de l'ombrage sur les services de régulation des bioagresseurs sont difficiles à établir, étant donné les effets antagonistes de l'ombrage sur ces biorégulateurs (chapitre 3). Dans le cadre du projet Safse, plusieurs approches ont été suivies pour mettre en évidence la régulation des bioagresseurs par l'ombrage dans les systèmes agroforestiers.

Pour étudier l'effet global de l'ombrage dans les systèmes agroforestiers, la première méthode proposée a consisté à décomposer cet effet sur les différentes parties du cycle de développement des bioagresseurs (chapitre 4). Cette méthode a été utilisée pour l'étude de l'effet de l'ombrage sur le pathosystème «rouille orangée-caféier». Lombrage a tendance à favoriser les processus pré-infectieux et ainsi l'incidence de la maladie. Mais, il diminuerait aussi la sévérité en favorisant l'effet des ennemis naturels (top-down). Lombrage et la diversité des arbres d'ombrage, caractérisés par leurs traits fonctionnels, auraient aussi des effets antagonistes sur la dispersion de la maladie (voir chapitre 3; Boudrot et al. 2016).

Une méthode complémentaire, fondée sur le développement de modèles épidémiologiques mécanistes de type sain-infecté-infectieux-retiré (SEIR $\left.{ }^{1}\right)$, a été utilisée pour étudier l'effet de l'ombrage sur le pathosystème Coffee berry disease (CBD) - caféier (cas $n^{\circ} 1$ ). Le CBD (anthracnose des baies) du caféier arabica, dû au champignon pathogène Colletotrichum kahawae, est un facteur de perte de récolte très important dans l'Ouest du Cameroun. Les arbres d'ombrage sont supposés réduire la dispersion par projection de propagules de la maladie (splashing) en formant une barrière contre la pluie (Mouen Bedimo et al., 2008), réduisant ainsi l'intensité de l'impact des gouttes de pluie sur les caféiers. Néanmoins, les données recueillies au cours de deux années consécutives dans l'Ouest du Cameroun montrent que l'ombrage ne réduit pas forcément la maladie et peut même la favoriser. Le modèle SEIR permet de compartimenter la population d'individus hôtes selon leur état et d'étudier les flux d'individus entre les compartiments (paramètres de changement d'états). Dans le modèle développé, certains paramètres de changement d'états sont des fonctions de covariables environnementales (température, humidité relative et quantité de pluie journalière) impliquées dans la dynamique de développement de la maladie telles que la germination et la dispersion du pathogène. Ce modèle a ensuite été couplé à un modèle probabiliste d'observation afin d'inférer les paramètres de changement d'états et de déterminer l'impact des covariables environnementales sur ces paramètres. Les premiers résultats suggèrent que les différences de développement de la maladie à l'ombre et en plein soleil s'expliqueraient principalement par une meilleure germination des propagules à l'ombre qu'en plein soleil. Ces résultats s'expliquent notamment par des conditions de température et humidité plus favorables à l'ombre. Ces approches épidémiologiques sont appropriées pour distinguer les mécanismes affectés par l'ombrage et choisir la meilleure stratégie à mettre en place pour optimiser le contrôle d'un bioagresseur particulier.

1. Statut des fruits dans le modèle. Un fruit peut être sain. Si le fruit est infecté, il devient infectieux. Il doit alors être retiré, soit parce qu'il tombe ou parce qu'on l'enlève. 


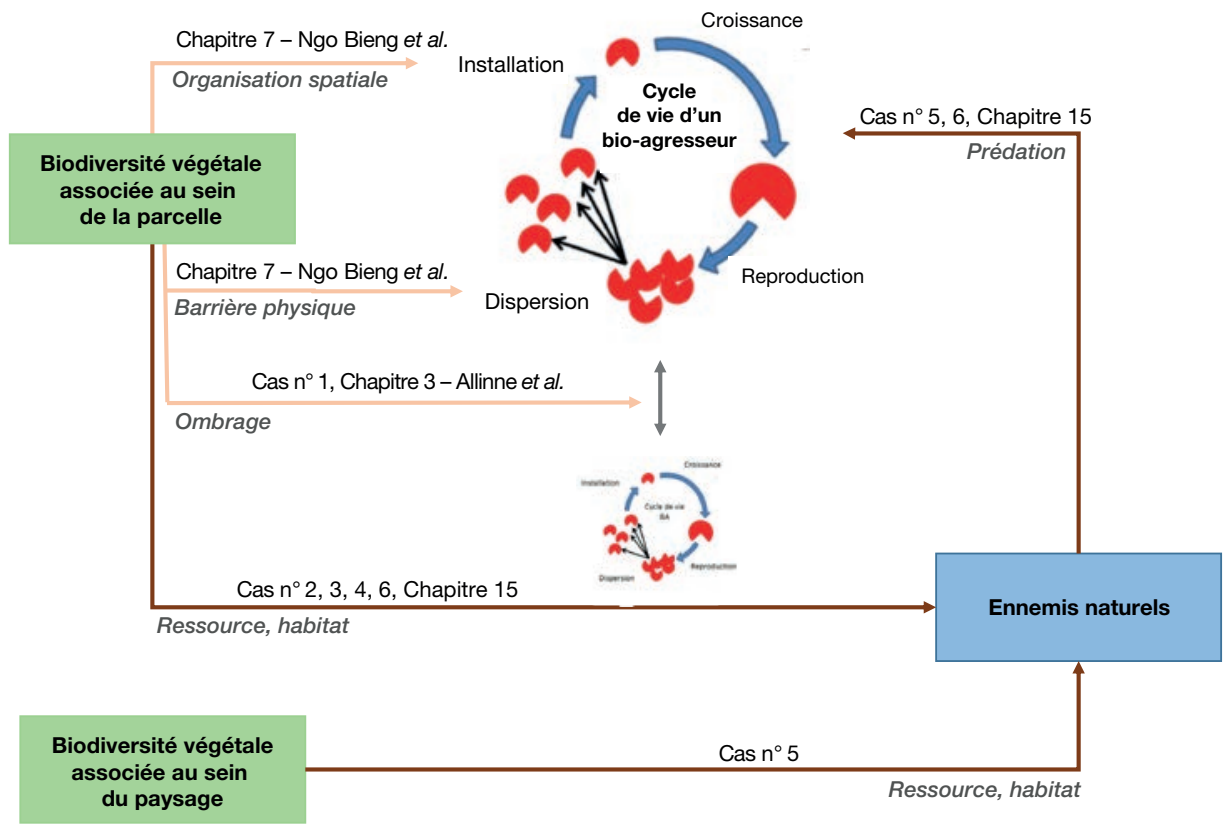

Figure 15.1. Synthèse de l'effet de la biodiversité végétale sur la régulation des bioagresseurs via les stratégies bottom-up et top-down à différentes échelles étudiées dans les cas d'étude.

Cas $n^{\circ} 1$, système agroforestier à base de caféiers, Cameroun; cas $n^{\circ} 2$ et $n^{\circ} 3$, système agroforestier à

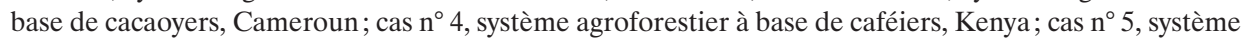
agroforestier à base de mil,; cas nº, système agroforestier à base de caféier, Costa Rica.

À l'échelle des systèmes agroforestiers, il existe souvent un bioagresseur principal et plusieurs bioagresseurs secondaires en termes d'impact sur la culture qui peuvent avoir des effets indésirables considérables s'ils sont cumulés. La composition de ce cortège de bioagresseurs varie en fonction des conditions climatiques et microclimatiques, elle peut être régulée par la gestion de l'ombrage (chapitre 3). Cette approche «multipest» permet de prendre en compte les effets des interactions, positives ou négatives, que peuvent avoir le système de culture sur l'ensemble du pathosystème. Elle est nécessaire pour aborder les relations dégâts - dommages dans les systèmes agroforestiers - et pour mettre en place des recommandations permettant de réduire les pertes de production dans ces systèmes.

\section{" Régulation top-down des bioagresseurs par la biodiversité végétale associée}

Une grande diversité végétale au sein d'un agroécosystème augmente généralement la diversité et l'abondance des ennemis naturels que sont les prédateurs ou les parasitoïdes des bioagresseurs de la culture principale (Thies et Tscharntke, 1999). C'est par exemple le cas dans les agroécosystèmes plurispécifiques, en comparaison des agroécosystèmes monospécifiques (Evans, 2016). Les plantes associées à la culture principale 
attirent les ennemis naturels des ravageurs en fournissant du pollen ou du nectar, qui sont une source d'alimentation. Elles peuvent aussi procurer un habitat favorable pour l'installation des ennemis naturels par la fourniture de proies ou d'hôtes alternatifs (Thies et Tscharntke, 1999). La présence de ces habitats dans le paysage est d'autant plus importante pour la biodiversité fonctionnelle que l'agroécosystème est simplifié et ne renferme pas une grande diversité de plantes associées à la culture principale, comme c'est souvent le cas dans les systèmes à base de cultures annuelles.

Choisis dans le cadre du projet Safse, les systèmes agroforestiers à base de plantes pérennes (caféiers ou cacaoyers) du Kenya, du Cameroun ou du Costa Rica contiennent un niveau de diversité végétale très important. Cette diversité attire une importante communauté d'ennemis naturels capables de réguler les populations de bioagresseurs. En se fondant sur cette hypothèse, l'évaluation des services de régulation fournis par ces systèmes agroforestiers à base de plantes pérennes s'est faite à l'échelle de la parcelle, sachant que les éléments paysagers ont un effet non négligeable sur les communautés d'ennemis naturels présents. À l'inverse, les systèmes de culture annuels généralement plus simples en termes de composition végétale, comme les systèmes à base de mil au Sénégal, nécessitent des échelles spatiales plus grandes pour atteindre le même degré de diversité et de complexité. Dans ce cas, l'étude des processus de régulation à l'échelle du paysage prend toute sa place. Cette démarche est aussi pertinente pour l'étude d'insectes ou d'agents pathogènes dotés d'une grande capacité de dispersion. Pour des organismes dont la dispersion est plus limitée, l'échelle de la plantation reste la plus appropriée pour étudier les services de régulation.

Dans le cadre du projet Safse, l'étude de la régulation naturelle des bioagresseurs dans les systèmes agroforestiers s'est faite en deux étapes. La première étape a consisté à établir le lien entre la composition végétale et les communautés d'ennemis naturels présentes. La deuxième étape a consisté à mettre en évidence l'efficacité de ces ennemis naturels à contrôler les bioagresseurs à partir des résultats des tests d'exclusion et d'étude des traits fonctionnels des ennemis naturels.

\section{Effet de la diversité végétale sur les communautés d'ennemis naturels}

Afin d'établir les liens entre la composition végétale et la composition en ennemis naturels des bioagresseurs, des approches d'écologie des communautés fondées sur l'étude des indices de diversité végétale, microbienne et animale à l'échelle de la parcelle ont été utilisées.

\section{Les systèmes agroforestiers à base de cacaoyers auCameroun}

La production du cacao est fortement contrainte par une maladie, la pourriture brune des cabosses causée par Phytophthora megakarya, et par un ravageur principal, Sahlbergella singularis (Miridae). Phytophthora megakarya est un agent pathogène qui attaque principalement les cabosses du cacaoyer. Il a une phase tellurique durant son cycle de développement, offrant des leviers d'action supplémentaires pour son contrôle. 
Champignons antagonistes des agents pathogènes et mycorhizes. Dans les systèmes agroforestiers, l'augmentation de l'humidité relative sous ombrage favorise le développement de la maladie. Néanmoins, les arbres d'ombrage pouvent potentiellement agir comme des réservoirs de souches de champignons. Leur présence pourrait aussi influencer la diversité et l'abondance dans le sol de champignons antagonistes des agents pathogènes, d'une part, et de mycorhizes, d'autre part, en facilitant la colonisation mycorhizienne des cultures adjacentes (Ingleby et al. 2007). Les mycorhizes peuvent, dans certains cas, conférer une protection contre les maladies : soit directement par la compétition, soit indirectement en induisant des résistances ou en stimulant la croissance de la plante (Ten Hoopen et Krauss, 2016). Tchameni et al. (2011) ont montré que les champignons mycorhiziens arbusculaires et le champignon Trichoderma asperellum augmentaient la croissance et la résistance du cacaoyer contre P. megakarya.

Diversité des mycorhizes et des antagonistes de $\boldsymbol{P}$. megakarya. Dans le cadre du projet Safse, l'effet de la diversité végétale - évaluée par la richesse spécifique, les indices de diversité et le type d'arbres - sur la diversité des mycorhizes et des antagonistes de $P$. megakarya dans le sol ont été étudiés (cas $\mathrm{n}^{\circ} 2$ ). L'hypothèse sous-jacente à cette activité est que les systèmes agroforestiers les plus riches en mycorhizes et en antagonistes auraient une meilleure résistance face à $P$. megakarya, se caractérisant par une incidence plus faible de la maladie dans les parcelles échantillonnées. Cette étude a été menée dans un réseau de 60 parcelles au sein desquelles des échantillons de terre ont été pris au pied des arbres et analysés en lien avec les caractéristiques physico-chimiques du sol. Le taux de mycorhization des cacaoyers a été significativement et positivement corrélé au taux de colonisation des racines des arbres associés dans toutes les parcelles étudiées; ce qui témoigne d'une «relation » entre la mycorhization des essences associées et la mycorhization des cacaoyers. Le genre Glomus a été le plus abondant. Au niveau des antagonistes, les genres Penicillium, Clonostachys et Trichoderma ont été les plus abondants. Le $\mathrm{pH}$ a été le facteur clé expliquant l'abondance des antagonistes, plus nombreux dans les sols de $\mathrm{pH}=6,5$ que dans les sols de $\mathrm{pH}=7$. Des analyses préliminaires ont montré que les systèmes abritant plus d'antagonistes ont été plus productifs (rendement en kg de fèves saines). Néanmoins, cette corrélation n'était pas significative.

Communautés de fourmis. Dans les systèmes agroforestiers, la biodiversité associée, en particulier les arbres d'ombrage, peut servir de refuge et fournir des ressources aux fourmis (Perfecto et al., 2014). Dans les systèmes agroforestiers à base de cacaoyers du Cameroun, les communautés de fourmis sont très diversifiées (Tadu et al., 2014). Les fourmis dominantes sont pour la plupart territoriales et colonisatrices. Cela peut avoir un effet négatif sur les autres groupes d'insectes qui partagent leur niche (prédation ou compétition par l'interférence pour l'espace). Plusieurs études ont mis en évidence le rôle de certaines espèces de fourmis, en particulier celles du genre Oecophylla, dans le contrôle de S. singularis (Ayenor et al., 2007; van Wijngaarden et al., 2007; Wielgoss et al., 2014; Bagny Beilhe et al., 2018).

Lactivité conduite dans le cadre du projet Safse a consisté à étudier l'effet de la composition végétale (indices de diversité et types d'espèces) des systèmes agroforestiers sur la diversité des communautés de fourmis pouvant avoir un rôle dans la régulation des ravageurs du cacaoyer $\left(\operatorname{cas}^{\circ} 3\right)$. Les activités se sont déroulées dans 
les plantations paysannes dans la région du Centre du Cameroun, dans une zone de $20 \mathrm{~km}^{2}$. La composition des systèmes suit un gradient de complexité :

- des systèmes en cultures pures;

- des systèmes agroforestiers traditionnels où le cacaoyer est associé à plus d'une dizaine d'espèces d'arbres forestiers et fruitiers;

- des systèmes agroforestiers plus simplifiés où le cacaoyer est associé à une ou deux espèces d'arbres à haute valeur ajoutée (arbres fruitiers, palmier à huile, cocotier selon un plan d'organisation bien défini et régulier).

Au cours des échantillonnages, un accent particulier a été mis sur les fourmis arboricoles qui occupent la même niche écologique que les mirides. Les indices de richesse et de diversité de la biodiversité végétale associée et des fourmis au sein des systèmes agroforestiers ont été calculés afin d'établir les liens entre ces variables à l'aide de modèles linaires généralisés. Une caractérisation de l'environnement proche des systèmes agroforestiers a aussi été réalisée.

Les approches développées n'ont pas permis d'établir des liens clairs entre les niveaux de diversité végétale et de diversité des fourmis, compte tenu des pratiques existant dans ces parcelles paysannes et de la complexité des interactions au sein des communautés de fourmis. Les résultats ont montré que la régulation des ravageurs du cacaoyer liée aux fourmis ne serait pas diminuée dans les systèmes avec peu de diversité végétale associée (monoculture ou système avec une espèce associée) car ils bénéficieraient de l'influence de l'environnement proche composé de savane, de forêt galerie, et de systèmes agroforestiers avec une forte diversité végétale.

\section{Les systèmes agroforestiers à base de caféiers au Kenya}

Une approche spatialisée a été utilisée dans des systèmes agroforestiers à base de caféiers pour étudier les services de régulation du scolyte des baies de café Hypothenemus hampei (Scolytidae), ravageur principal du café (cas $\mathrm{n}^{\circ} 4$ ) à l'échelle mondiale (Vega et al., 2009).

Les liens entre la diversité végétale, l'abondance des ravageurs et la diversité des parasitoïdes ont été établis à partir de corrélations spatiales. Dans la région de Murang'a, l'une des principales zones de production du café Arabica au Kenya, le café est souvent cultivé en association avec des arbres, en particulier Grevillea robusta, et de nombreuses espèces d'arbres fruitiers et forestiers. Les plantations de café de la zone sont fortement infestées par H. hampei, en particulier dans la zone basse de culture, entre 1300 et 1500 m d'altitude. Dans cette région, il existe une grande diversité d'ennemis naturels du scolyte des baies, principalement des hyménoptères parasitoïdes qui se développent aux dépens de ses oufs et de ses larves à l'intérieur des baies de café. Néanmoins, les processus qui favorisent leur présence n'ont pas été étudiés. Une évaluation spatio-temporelle des niveaux d'infestation par le scolyte et du taux de parasitisme a été réalisée à l'échelle de la parcelle. La distribution spatiale des scolytes est fortement structurée, alors que celle de l'espèce majoritaire de parasitoïde Prorops nasuta (Bethylidae) ne semble pas l'être. Étant donné les faibles effectifs des parasitoïdes, il n'a pas été possible d'établir des corrélations spatiales entre la présence des arbres et les taux de parasitisme. 
Ce type d'approches fondées sur l'étude des facteurs environnementaux favorisant la présence d'ennemis naturels est important pour bien connaître la composition et la structure des communautés en lien avec la structure et la composition des systèmes agroforestiers. Mais, elles ne permettent pas réellement d'évaluer l'efficacité de la régulation naturelle dans ces systèmes pour réduire les populations de bioagresseurs. Des approches expérimentales ou d'écologie fonctionnelle sont nécessaires pour réellement identifier les leviers d'action possibles dans les systèmes agroforestiers.

\section{Effet de la diversité végétale sur la régulation top-down des bioagresseurs}

\section{Les systèmes de culture à base de céréales et de légumineuses au Sénégal}

La mineuse de l'épi, Heliocheilus albipunctella (Noctuidae), représente une contrainte majeure à l'intensification de la production de mil, causant jusqu'à $85 \%$ de perte de rendement en grains. En l'absence de traitement insecticide sur le mil, l'action des ennemis naturels est une composante majeure de la régulation des populations du ravageur. Dans ces zones, l'agriculture est dominée par des systèmes de culture à base de céréales (mil) et de légumineuses (arachide et niébé). Les associations permanentes avec des arbres (Faidherbia albida) constituent une des solutions pour faire face au défi de l'intensification des systèmes de production et à la préservation de la biodiversité. Si l'amélioration de la fertilité des sols permise par ces systèmes agroforestiers a été démontrée, certains services de régulation, comme le contrôle des bioagresseurs, ont été très peu étudiés. Grâce à une structure complexe composée de strates et de milieux différents, les systèmes agroforestiers offrent aux arthropodes une grande diversité de niches écologiques dans le temps et dans l'espace qu'une simple mosaïque de cultures annuelles. Ils peuvent ainsi jouer un rôle de source ou de relais pour les populations de bioagresseurs, ou favoriser l'impact des ennemis naturels en leur offrant des ressources complémentaires, en particulier pendant la saison sèche.

Au sein du paysage. La gestion de ces habitats au sein du paysage constitue un levier pour favoriser la régulation naturelle des populations de bioagresseurs. Une première étape a donc consisté en l'identification des éléments du paysage, dont la végétation naturelle intervenant dans la régulation des populations de la mineuse de l'épi par les ennemis naturels (cas $\left.n^{\circ} 5\right)$. Des images à très haute résolution spatiale (THRS) ont été utilisées pour cartographier et quantifier les éléments clés du paysage sur un territoire du bassin arachidier. De $20 \times 20 \mathrm{~km}^{2}$, ce paysage est centré sur le village de Dangalma dans la région de Diourbel (Soti et al., 2018). Cinq éléments du paysage susceptibles d'affecter la régulation des populations de la mineuse de l'épi ont été retenus : l'indice d'abondance des parcelles de mil, l'indice de densité des arbres, l'indice de diversité de Shannon, l'indice de proximité des parcelles de mil et l'indice de proximité des parcelles d'arbres. Ces indices ont été calculés pour chaque année d'observation pendant deux ans à neuf échelles spatiales différentes, dans un rayon de 0,25 à 2,25 km autour des 90 parcelles de mil (unité d'échantillonnage). 
Régulation naturelle de la mineuse du mil. En parallèle, la régulation naturelle de la mineuse du mil a été estimée expérimentalement à partir des manchons d'exclusion des ennemis naturels posés sur des épis-sentinelles infestés dans 45 parcelles d'observation (calcul du Biocontrol service index, BSI par Gardiner et al., 2009). Pour chaque rayon autour des parcelles de mil, des modèles explicatifs de type modèle linéaire généralisé (GLM) ont permis d'identifier les variables paysagères ayant un effet sur la régulation naturelle. Les meilleurs modèles ont été sélectionnés sur la base de l'AIC (Akaike information criterion). Le modèle composé des indices de diversité des arbres - l'indice de diversité de Shannon et l'indice d'abondance des parcelles de mil dans un rayon de $1,75 \mathrm{~km}$ autour des parcelles de mil — s'est avéré le plus pertinent pour expliquer la régulation naturelle de la mineuse du mil. En outre, le Biocontrol service index augmente avec les indices de densité des arbres et de diversité de Shannon. Il diminue lorsque le paysage est dominé par des cultures de mil. Labondance des habitats semi-naturels (contenant des arbres) au sein du paysage offrirait des habitats favorables aux ennemis naturels de la mineuse des épis et augmenterait le control biologique de ce ravageur (Thiaw et al., 2017; Soti et al., 2019).

\section{Les systèmes agroforestiers à base de caféiers auCosta Rica}

Une approche basée sur l'étude des traits fonctionnels des oiseaux a été développée pour étudier leur effet sur le contrôle du scolyte des baies (H. hampei) dans les systèmes agroforestiers à base de caféiers (Martínez-Salinas et al., 2016) (cas $\left.\mathrm{n}^{\circ} 6\right)$. Un trait fonctionnel est une caractéristique mesurable d'un individu donné incluant des caractéristiques morphologiques, physiologiques, phénologiques ou comportementales.

Rôle des oiseaux insectivores. Des études récentes ont montré que des espèces d'oiseaux insectivores joueraient un rôle important dans le contrôle de ce ravageur (Karp et al., 2013; Kellermann et al., 2008). Karp et al., (2013) ont notamment démontré la contribution des habitats adjacents (comme la forêt) pour renforcer le contrôle de ce ravageur via des effets de colonisation d'habitats à partir de parcelles adjacentes (spill-over). Cependant, le lien entre ces parcelles de forêts résiduels et les oiseaux, qui sont réellement des prédateurs de H. hampei, reste à établir. Au sein d'une communauté, les traits fonctionnels permettent d'étudier la valeur, la gamme, la distribution et l'abondance des traits des espèces qui la constituent.

Une étude a été menée pour évaluer l'effet des arbres d'ombrage sur la diversité fonctionnelle (DF) des oiseaux et l'effet de cette diversité aviaire sur l'infestation du scolyte des baies. Cette activité s'est déroulée en parcelle d'expérimentation au Catie, au Costa Rica. Dix caféiers ont été placés en cage pour exclure la prédation des oiseaux et dix autres caféiers voisins non protégés ont été suivis. Les niveaux d'infestation naturelle et manipulée (en plaçant des fruits infestés au pied des arbres suivis) ont été observés (Martínez-Salinas et al., 2016). Des captures d'oiseaux ont été réalisées sur les dix emplacements. Il a été mis en évidence une interaction entre la période de l'année considérée et la couverture d'ombrage pour la diversité fonctionnelle aviaire. Par ailleurs, il a été prouvé que la diversité fonctionnelle aviaire est un prédicteur majeur du taux d'infestation du scolyte. 
Il a donc été possible d'établir que la couverture d'ombrage intervenait dans les services de régulation des populations de scolyte via son action sur la diversité fonctionnelle des oiseaux. Dans ce contexte, la taille des arbres d'ombrage qui a lieu en milieu d'année, au moment où la plupart des scolytes sont en position de perforation des cerises de café, aurait tendance à réduire la régulation naturelle de ce ravageur par les oiseaux. Létude de la diversité fonctionnelle au sein des communautés, qui colonisent les systèmes agroforestiers, est importante pour optimiser la gestion de ces systèmes agroforestiers et favoriser la conservation d'espèces utiles en leur sein.

\section{Conclusion et perspectives}

Les approches décrites dans nos différents cas d'études ont bien illustré que les services de régulation des bioagresseurs fournis par la biodiversité végétale dans les systèmes agroforestiers étaient multiples et relevaient de processus complexes. Pour le développement de stratégies agroécologiques de gestion des bioagresseurs, une étape importante est l'intégration de ces connaissances dans des modèles de fonctionnement des systèmes agroforestiers prenant en compte les interactions avec l'environnement abiotique et entre communautés. Cette connaissance est indispensable pour actionner les leviers permettant d'optimiser ou de piloter les processus de régulation des bioagresseurs dans les systèmes agroforestiers (Gaba et al., 2014; Gaba et al., 2015).

L'emploi d'outils statistiques appropriés - tels que les analyses canoniques des redondances, les méthodes d'équations structurelles, les méthodes de régression par les moindres carrés partiels) — permettent d'expliquer et d'intégrer les interactions complexes dans les systèmes agroforestiers. Ces outils sont utilisés pour identifier les contributions relatives et jointes des mécanismes de régulation à différentes échelles (parcelle ou paysage) (voir chapitres 3 et 7; Gidoin et al., 2014). Ils pourraient servir de base à une première étape d'analyse des différentes composantes des services de régulation dans les systèmes agroforestiers, puis de déduction des leviers potentiels pour optimiser la gestion des bioagresseurs.

Si l'effet de la biodiversité végétale sur la régulation des bioagresseurs commence à être bien étudié, la relation plus complexe entre cette biodiversité et les pertes de production causées par les bioagresseurs a été peu étudiée dans le cas des systèmes agroforestiers complexes à base de cultures pérennes.

Pour optimiser la régulation naturelle des bioagresseurs au sein des systèmes agroforestiers et contrebalancer l'effet net sur les pertes de production, il faudrait considérer les compromis entre les services écosystèmiques au sein des systèmes (chapitre 4; Allinne et al., 2016). La compréhension et la gestion des interactions entre différents services devraient permettre d'obtenir des bénéfices importants pour les rendements à partir d'un investissement réduit en termes de gestion.

Les cas d'études présentés ici ont surtout servi à mettre en évidence des effets de la diversité végétale sur la régulation des maladies et ravageurs (de manière très globale). Mais, les résultats ne sont pas toujours tranchés car il y a beaucoup d'interactions à prendre en compte. 


\section{- Bibliographie}

Allinne C., Savary S., Avelino J., 2016. Delicate balance between pest and disease injuries, yield performance, and other ecosystem services in the complex coffee-based systems of Costa Rica. Agriculture, ecosystems and environment, 222:1-12. DoiI: http://dx.doi.org/10.1016/j.agee.2016.02.001

Avelino J., Ten Hoopen G.M., Declerck F., 2011. Ecological mechanisms for pest and disease control in coffee and cacao agroecosystems of the Neotropics. In : Rapidel Bruno, DeClerck Fabrice, Le Coq Jean-François, Beer John (eds). Ecosystem services from agriculture and agroforestry: measurement and payment. Londres, Royaume Uni: Earthscan Publications, 91-117.

Ayenor G.K., Huis A.V., Obeng O.D., Padi B., Roling N.G., 2007. Facilitating the use of alternative capsid control methods towards sustainable production of organic cocoa in Ghana. International journal of tropical insect science, 27: 85-94.

Bagny Beilhe L., Piou C., Tadu Z., Babin R., 2018. Identifying Ant-Mirid Spatial Interactions to Improve Biological Control in Cacao-Based Agroforestry System. Environmental entomology, 47: 551-558.

Beer J., Muschler R., Kass D., Somarriba E., 1997. Shade management in coffee and cacao plantations. Agroforestry systems, 38: 139-164.

Evans E.W., 2016. Biodiversity, ecosystem functioning, and classical biological control. Applied entomology and zoology, 51: 173-184. Doi: 10.1007/s13355-016-0401-z

Gaba S., Bretagnolle F., Rigaud T., Philippot L., 2014. Managing biotic interactions for ecological intensification of agroecosystem. Frontiers in ecology and evolution, 2. Doi: 10.3389/fevo.2014.00029

Gaba S., Lescourret F., Boudsocq S., Enjalbert J., Hinsinger P., Journet E.P., Navas M.L., Wery J., Louarn G., Malezieux E., Pelzer E., Prudent M., Ozier-Lafontaine H., 2015. Multiple cropping systems as drivers for providing multiple ecosystem services: from concepts to design. Agronomy for sustainable development, 35: 607-623. Doi: 10.1007/s13593-014-0272-z

Gardiner M., Landis D., Gratton C., DiFonzo C., O'neal M., Chacon J., Wayo M., Schmidt N., Mueller E., Heimpel G., 2009. Landscape diversity enhances biological control of an introduced crop pest in the northcentral USA. Ecological applications, 19: 143-154.

Gidoin C., Avelino J., Deheuvels O., Cilas C., Bieng M.A.N., 2013. Shade tree spatial structure and pod production explain frosty pod rot intensity in cacao agroforests, Costa Rica. Phytopathology, 104: 275-281. Doi: 10.1094/phyto-07-13-0216-r

Gidoin C., Babin R., Bagny Beilhe L., Cilas C., ten Hoopen G.M., Bieng M.A., 2014. Tree spatial structure, host composition and resource availability influence mirid density or black pod prevalence in cacao agroforests in Cameroon. PLoS one, 9:e109405. Doi: 10.1371/journal.pone.0109405 PONE-D-14-17577 (pii)

Ingleby K., Wilson J., Munro R.C., Cavers C., 2007. Mycorrhizas in Agroforestry: spread and sharing of arbuscular mycorrhizal fungi between trees and crops: complementary use of molecular and microscopic approaches. Plant and soil, 294: 125-136.

Karp D.S., Mendenhall C.D., Sandí R.F., Chaumont N., Ehrlich P.R., Hadly E.A., Daily G.C., 2013. Forest bolsters bird abundance, pest control and coffee yield. Ecology letters, 16: 1339-1 347. Doi: $10.1111 /$ ele.12173

Kellermann J.L., Johnson M.D., Stercho A.M., Hackett S.C., 2008. Ecological and economic services provided by birds on Jamaican Blue Mountain coffee farms. Conservation biology, 22: 1 177-1 185 .

Malézieux E., 2012. Designing cropping systems from nature. Agronomy for sustainable development, 32 (1): 15-29.

Martínez-Salinas A., DeClerck F., Vierling K., Vierling L., Legal L., Vílchez-Mendoza S., Avelino J., 2016. Bird functional diversity supports pest control services in a Costa Rican coffee farm. Agriculture, ecosystems and environment, 235: 277-288.

Mouen Bedimo J.A., Njiayouom I., Bieysse D., Ndoumbe Nkeng M., Cilas C., Notteghem J.L., 2008. Effect of shade on Arabica coffee berry disease development: Toward an agroforestry system to reduce disease impact. Phytopathology, 98: 1320-1 325. Doi: 10.1094/phyto-98-12-1320 
Perfecto I., Vandermeer J., Philpott S.M., 2014. Complex ecological interactions in the coffee agroecosystem. Annual review of ecology, evolution, and systematics, 45: 137-158.

Ratnadass A., Fernandes P., Avelino J., Habib R., 2012. Plant species diversity for sustainable management of crop pests and diseases in agroecosystems: a review. Agronomy for sustainable development, 32: 272-303.

Schroth G., Krauss U., Gasparotto L., Aguilar J.A.D., Vohland K., 2000. Pests and diseases in agroforestry systems of the humid tropics. Agroforestry systems, 50: 199-241. Doi: 10.1023/a:1006468103914

Soti V., Lelong C., Goebel F.R., Brévault T., 2018. Designing a field sampling plan for landscapepest ecological studies using VHR optical imagery. International Journal of applied earth observation and geoinformation, 72: 26-33.

Soti V., Thiaw I., Debaly Z.M., Sow A., Diaw M., Fofana S., Diakhate M., Thiaw C., Brévault T., 2019. Effect of landscape diversity and crop management on the control of the millet head miner, Heliocheilus albipunctella (Lepidoptera: Noctuidae) by natural enemies. Biological control, 129: 115-122. Doi.org/10.1016/j.biocontrol.2018.10.006

Tadu Z., Djiéto-Lordon C., Yede, Youbi E.M., Aléné C.D., Fomena A., Babin R., 2014. Ant mosaics in cocoa agroforestry systems of Southern Cameroon: influence of shade on the occurrence and spatial distribution of dominant ants. Agroforestry systems, 88: 1 067-1 079. Doi: 10.1007/ s10457-014-9676-7

Tchameni S., Ngonkeu M., Begoude B., Nana L.W., Fokom R., Owona A., Mbarga J., Tchana T., Tondje P., Etoa F., 2011. Effect of Trichoderma asperellum and arbuscular mycorrhizal fungi on cacao growth and resistance against black pod disease. Crop protection, 30: 1 321-1 327.

Ten Hoopen G.M., Krauss U., 2016. Biological control of cacao diseases. In: Bailey Bryan A., Meinhardt Lyndel W. (eds). Cacao diseases: a history of old enemies and new encounters. Cham : Springer International Publishing, 511-566.

Thiaw I., Soti V., Goebel F.R., Sow A., Brevault T., Diakhate M., 2017. Effect of landscape diversity on biocontrol of the millet head miner, Heliocheilus albipunctella (Lepidoptera: Noctuidae) in Senegal. IOBC/WPRS bulletin, 122: 38-42.

Thies C., Tscharntke T., 1999. Landscape structure and biological control in agroecosystems. Science, 285: 893-895. Doi: 7732 (pii)

van Wijngaarden P.M., van Kessel M., van Huis A., 2007. Oecophylla longinoda (Hymenoptera: Formicidae) as a biological control agent for cocoa capsids (Hemiptera: Miridae). Netherlands entomological society meeting, 18: 21-30.

Vega F.E., Infante F., Castillo A., Jaramillo J., 2009. The coffee berry borer, Hypothenemus hampei Ferrari (Coleoptera: Curculionidae): a short review, with recent findings and future research directions. Terrestrial arthropod reviews, 129-147.

Wielgoss A., Tscharntke T., Rumede A., Fiala B., Seidel H., Shahabuddin S., Clough Y., 2014. Interaction complexity matters: disentangling services and disservices of ant communities driving yield in tropical agroecosystems. Proceedings of the royal society of London B: biological sciences, 281. Doi: $10.1098 / \mathrm{rspb} .2013 .2144$ 


\section{Liste des auteurs}

\section{Chapitre 1}

\section{Baranger Marie}

Cirad, Inra, Montpellier SupAgro,

Université de Montpellier, UMR-Art-Dev,

Montpellier, France

mariebaranger@gmail.com

\section{Constanty Manon}

Cirad, Inra, Montpellier SupAgro, Université de Montpellier, UMR-Innovation, Montpellier, France; AgroParisTech UMR-1048 Sadapt, Paris, France constanty.manon@gmail.com

\section{Dhorne Soazic}

Cirad, Inra, Montpellier SupAgro, Université de Montpellier, UMR-Innovation, Montpellier, France; AgroParisTech UMR-1048 Sadapt Paris, France soazic.dhorne@gmail.com

\section{Dorgans-Cadilhac Jeffry}

Cirad, Inra, Montpellier SupAgro, Université de Montpellier, UMR-Art-Dev, Montpellier, France j.dorgans@outlook.com

\section{Freguin-Gresh Sandrine}

Cirad, Inra, Montpellier SupAgro,

Université de Montpellier, UMR-Art-Dev,

Montpellier, France

sandrine.freguin@cirad.fr

\section{Gutiérrez Montes Isabel}

Catie, 7170 Turrialba, Costa Rica 30501

igutie@catie.ac.cr

\author{
Laffourcade Roland \\ Cirad, Inra, Montpellier SupAgro, \\ Université de Montpellier, UMR-Innovation, \\ Montpellier, France; AgroParisTech \\ UMR-1048 Sadapt Paris, France \\ roland.laffourcade@yahoo.fr \\ Le Coq Jean-François \\ Cirad, Inra, Montpellier SupAgro, \\ Université de Montpellier, UMR-Art-Dev, \\ Montpellier, France; \\ CINPE, UNA, Heredia, Costa Rica \\ jean-francois.le_coq@cirad.fr, \\ JF.LeCoq@CGIAR.ORG

\section{Sibelet Nicole} \\ Cirad, Inra, Montpellier SupAgro, \\ Université de Montpellier, UMR-Innovation, \\ Montpellier, France; \\ Catie, 7170 Turrialba, Costa Rica \\ nicole.sibelet@cirad.fr
}

\section{Chapitre 2}

\section{Albrecht Alain \\ Cirad, IRD, Université Montpellier, UMR-Eco\&Sols, Montpellier, France alain.albrecht@ird.fr}

\footnotetext{
Allinne Clémentine

Cirad, Ciheam-Iamm, Inra, Montpellier SupAgro, Université de Montpellier, UMR-System, Montpellier, France; Catie, Programma de agricultura, ganaderia y agroforesteria, Turrialba, Costa Rica clementine.allinne@cirad.fr
} 


\section{Acuna Vargas R.}

Hacienda Aquiares, Costa Rica racuna@aquiares.com

\section{Avelino Jacques}

Cirad, Université de Montpellier, UPR-Bioagresseurs, Montpellier, France;

Catie, Programma de agricultura, ganaderia y agroforesteria, Turrialba, Costa Rica jacques.avelino@cirad.fr

\section{Barquero A.}

Hacienda Aquiares, Costa Rica alebarquero2@gmail.com

\section{Barthès Bernard}

Cirad, IRD, Université Montpellier,

UMR-Eco\&Sols, Montpellier, France bernard. barthes@ird.fr

\section{Benegas Laura}

Catie, 7170 Turrialba, Costa Rica;

Swedish University of Agricultural Sciences

(SLU), Suède

Laura.Benegas@slu.se,

laura.benegas@catie.ac.cr

\section{Bonnefond Jean-Marc}

Inra, UMR-ISPA, Bordeaux, France

jean-marc.bonnefond@inra.fr

\section{Borgonovo C.}

Hacienda Aquiares, Costa Rica

cborgonovo4@gmail.com

\section{Cambou Aurélie}

Cirad, IRD, Université Montpellier,

UMR-Eco\&Sols, Montpellier, France;

Agrocampus Ouest, UPSP EPHor,

Angers, France

aurelie.cambou5@gmail.com

\section{Charbonnier F.}

Cirad, IRD, Université Montpellier,

UMR-Eco\&Sols, Montpellier, France;

Catie, 7170 Turrialba, Costa Rica; PCP

Agroforestry MesoAmerica, Catie, Costa

Rica; El Colegio de la Frontera Sur,

SCddc, Chiapas, México

charbonnier@ecosur.mx

\section{Chevallier Tiphaine}

Cirad, IRD, Université Montpellier, UMR-Eco\&Sols, Montpellier, France tiphaine.chevallier@ird.fr

\section{Clément-Vidal Anne}

Cirad, UMR-AGAP, Montpellier, France anne. clement-vidal@cirad.fr

\section{Dauzat Jean}

Cirad, Inra, UMR-Amap, Montpellier, France jean.dauzat@cirad.fr

\section{Defrenet Elsa}

Agrocampus Ouest, Rennes, France

elsa.defrenet@gmail.com

\section{de Melo Virginio Filho Elias}

Catie, 7170 Turrialba, Costa Rica; PCP

Agroforestry MesoAmerica, Catie, Costa Rica eliasdem@catie.ac.cr

\section{Do Frederic}

Cirad, IRD, Université Montpellier,

UMR-Eco\&Sols, Montpellier, France

frederic.do@ird.fr

\section{Dreyer Erwin}

Inra, UMR-EEF, Inra-Nancy, France

erwin.dreyer@inra.fr

\section{Duthoit Maxime}

Cirad, IRD, Université Montpellier, UMR-Eco\&Sols, Montpellier, France maxime.duthoit@cirad.fr

\section{Eitel J.}

University of Idaho, États-Unis

jeitel@uidaho.edu

\section{Elberling B.}

University Copenhagen, Copenhagen,

Danemark

be@ign.ku.dk

Fonseca C.

Icafe, Costa Rica

cfonseca@icafe.cr

\section{Gay Frédéric}

Cirad, IRD, Université Montpellier,

UMR-Eco\&Sols, Montpellier, France;

Catie, 7170 Turrialba, Costa Rica; PCP

Agroforestry MesoAmerica, Catie, Costa Rica frederic.gay@cirad.fr

\section{Gómez-Delgado F.}

Cirad, IRD, Université Montpellier, UMR-Eco\&Sols, Montpellier, France; ICE - Costa Rican Institute of Electricity, Costa Rica; PCP Agroforestry MesoAmerica, Catie, Costa Rica; World Meteorological Organization (WMO), Costa Rica eduardogranados30@gmail.com

\section{Guidat Florian}

Cirad, IRD, Université Montpellier, UMR-Eco\&Sols, Montpellier, France florian.guidat22@gmail.com 


\section{Harmand Jean-Michel}

Cirad, IRD, Université Montpellier, UMR-Eco\&Sols, Montpellier, France; Icraf (World Agroforestry) Nairobi, Kenya jean-michel.harmand@cirad.fr

\section{Isaac Marney}

DPES, University of Toronto, Scarborough, Canada

marney.isaac@utoronto.ca

\section{Jara Manuel}

Hacienda Aquiares, Costa Rica

manueljaraq@yahoo.es

\section{Jourdan Christophe}

Cirad, IRD, Université Montpellier, UMR-Eco\&Sols, Montpellier, France;

Inra, Montpellier SupAgro,

UMR-Lisah, France

christophe.jourdan@cirad.fr

\section{Kim J.}

Cirad, Inra, UMR-Amap, Montpellier, France jkim@bgc-jena.mpg.de

\section{Kinoshita R.}

Cornell University, États-Unis

rk422@cornell.edu

\section{Le Bissonnais Yves}

Inra, Montpellier SupAgro, UMR-Lisah,

Montpellier, France

lebisson@supagro.inra.fr

\section{Lehner Peter6hans}

Hacienda Aquiares, Costa Rica

peterhanslehner@gmail.com

\section{Le Maire Guerric}

Cirad, IRD, Université Montpellier, UMR-Eco\&Sols, Montpellier, France guerric.le_maire@cirad.fr

\section{Loustau Denis}

Inra, UMR-ISPA, Bordeaux, France

denis.loustau@inra.fr

\section{Madsen Mikael}

University Copenhagen, Copenhagen,

Danemark

Mikael.Madsen@jur.ku.dk

\section{Mages C.}

University Copenhagen, Copenhagen,

Danemark

fmz403@alumni.ku.dk

\section{Malmer Anders}

Swedish University of Agricultural Sciences

(SLU), Suède

anders.malmer@slu.se

\section{Martin Adam \\ DPES, University of Toronto, Scarborough, Canada \\ adam.martin@utoronto.ca \\ Moussa Rachida \\ Inra, Montpellier SupAgro, UMR-Lisah, Montpellier France \\ moussa@supagro.inra.fr}

\section{Nespoulous Jérôme}

Cirad, Inra, UMR-Amap, Montpellier, France jerome.nespoulous@cirad.fr

\section{Pérez Molina J.P.}

Catie, 7170 Turrialba, Costa Rica; PCP Agroforestry MesoAmerica, Catie, Costa Rica; Federal University of Viçosa, Brazil; Montpellier SupAgro, Montpellier, France jpastorpm@gmail.com

\section{Picart Delphine}

Inra, UMR-Ispa, Bordeaux, France delphine.deshors-picart@inra.fr

\section{Priemé A.}

University Copenhagen, Copenhagen,

Danemark

aprieme@bio.ku.dk

\section{Prieto Ivan}

CNRS, Cefe UMR-5 175, Montpellier, France ivan.prieto@cefe.cnrs.fr

\section{Ramirez Guillermo}

Hacienda Aquiares, Costa Rica guiller2151@yahoo.es

\section{Rançon Florian}

Cirad, IRD, Université Montpellier, UMR-Eco\&Sols, Montpellier, France florian.rancon@ims-bordeaux.fr

\section{Rapidel Bruno}

Cirad, Ciheam-Iamm, Inra, Montpellier

SupAgro, Université de Montpellier, UMR-System, Montpellier, France; PCP Agroforestry MesoAmerica, Catie, Costa Rica bruno.rapidel@cirad.fr

\section{Robelo D.}

Hacienda Aquiares, Costa Rica drobelo@aquiares.com 


\section{Rocheteau Alain}

Cirad, IRD, Université Montpellier, UMR-Eco\&Sols, Montpellier, France alain.rocheteau@ird.fr

\section{Roumet Catherine}

CNRS, Cefe UMR-5175, Montpellier, France catherine.roumet@cefe.cnrs.fr

\section{Roupsard Olivier}

Cirad, IRD, Université Montpellier, UMR-Eco\&Sols, Montpellier, France; Catie, 7170 Turrialba, Costa Rica; PCP Agroforestry MesoAmerica, Catie, Costa Rica LMI IESOL (IRD/Isra/Ucad/Cirad), Dakar, Sénégal olivier.roupsard@cirad.fr

\section{Saint-André L.}

Cirad, IRD, Université Montpellier, UMR-Eco\&Sols, Montpellier, France; Inra, UR-BEF, Nancy, France st-andre@nancy.inra.fr

\section{Sánchez-Murillo Ricardo}

UNA, Stable Isotope Research Group, Costa Rica

ricardo.sanchez.murillo@una.cr

\section{Schnabel Florian}

Catie, 7170 Turrialba, Costa Rica; FENR, University of Freiburg, Allemagne florian.schnabel@idiv.de

\section{Soma Maxime \\ Cirad, IRD, Université Montpellier, UMR-Eco\&Sols, Montpellier, France; UR-Écologie des Forêts Méditerranéennes, Inra, Avignon, France maxime.soma@inra.fr}

\section{Stokes Alexia}

Cirad, Inra, UMR-Amap, Montpellier, France alexia.stokes@cirad.fr

\section{Taugourdeau Simon}

Cirad, UMR-Selmet, Montpellier, France simon.taugourdeau@cirad.fr

\section{Vaast Philippe}

Cirad, IRD, Université Montpellier, UMR-Eco\&Sols, Montpellier, France; Icraf (World Agroforestry) Nairobi, Kenya philippe.vaast@cirad.fr

\section{Valentin Christian} IRD, UMR-IEES, Paris, France christian.valentin@ird.fr

\section{Van den Meersche Karel}

Cirad, IRD, Université Montpellier, UMR-Eco\&Sols, Montpellier, France; Catie, 7170 Turrialba, Costa Rica; PCP Agroforestry MesoAmerica, Catie, Costa Rica karel.van_den_meersche@cirad.fr

\section{Vezy Rémi}

Université de Bordeaux, Bordeaux, France; Cirad, IRD, Université Montpellier, UMR-Eco\&Sols, Montpellier, France remi.vezy@inra.fr, remi.vezy@cirad.fr

\section{Vierling Lee \\ University of Idaho, États-Unis; Catie, 7170 Turrialba, Costa Rica leev@uidaho.edu}

\section{Welsh Kristen \\ Catie, 7170 Turrialba, Costa Rica; \\ University of Idaho, États-Unis \\ kristen.unwala@ub.edu.bs}

\section{Chapitre 3}

\section{Allinne Clémentine}

Cirad, Ciheam-Iamm, Inra, Montpellier SupAgro, Université de Montpellier, UMR-System, Montpellier, France; Catie, Programma de agricultura, ganaderia y agroforesteria, Turrialba, Costa Rica clementine.allinne@cirad.fr

\section{Avelino Jacques}

Cirad, Université de Montpellier, UPR-Bioagresseurs, Montpellier, France; Catie, Programma de agricultura, ganaderia y agroforesteria, Turrialba, Costa Rica; IicaPromecafe, Coronado, San José, Costa Rica jacques.avelino@cirad.fr

\section{Boudrot Audrey}

Cirad, Université de Montpellier, UPR-Bioagresseurs, Montpellier, France; Agrocampus Ouest, Rennes, France audrey.boudrot@adear37.org,

\section{De Melo Elias}

Catie, Programma de agricultura, ganaderia y agroforesteria, Turrialba, Costa Rica eliasdem@catie.ac.cr

\section{Granados Eduardo}

Universidad de Costa Rica, sede del Atlántico, Turrialba, Costa Rica eduardogranados30@gmail.com 


\section{Merle Isabelle}

Cirad, Université de Montpellier,

UPR-Bioagresseurs, Montpellier, France;

Ensaia, Protection des cultures, Vandæuvre-

lès-Nancy, France

isabelle.merle@cirad.fr

\section{Pico Jimmy}

Cirad, Université de Montpellier,

UPR-Bioagresseurs, Montpellier, France;

Catie, Programma de agricultura, ganaderia y

agroforesteria, Turrialba, Costa Rica

jimmy.pico@iniap.gob.ec

\section{Vonthron Simon}

Cirad, Inra, Montpellier SupAgro, Université de Montpellier, Montpellier, France

simon.vonthron@supagro.inra.fr, simon.

vonthron@acadis.org

\section{Chapitre 4}

\section{Aucante Mélaine}

Istom, Angers, France; AgroParisTech, Cirad, IRD, Irstea, Université de Montpellier, UMR-G-EAU, Montpellier, France melaine.aucante@irstea.fr

\section{Bonifazi Mathilde}

IRC, Montpellier SupAgro, France

mathilde.bonifazi@inra.fr

\section{Ferrand Nils}

AgroParisTech, Cirad, IRD, Irstea, Université de Montpellier, UMR-G-EAU, Montpellier,

Francee

nils.ferrand@irstea.fr

\section{Freguin-Gresh Sandrine}

Cirad, Inra, Montpellier SupAgro, Université de Montpellier, UMR-Art-Dev, Montpellier, France

sandrine.freguin@cirad.fr

\section{Le Coq Jean-François}

Cirad, Inra, Montpellier SupAgro, Université de Montpellier, UMR-Art-Dev, Montpellier, France; Ciat, Cali, Colombie; CINPE, UNA, Heredia, Costa Rica

jean-francois.le_coq@cirad.fr, JF.LeCoq@ CGIAR.ORG

\section{Rapidel Bruno}

Cirad, Ciheam-Iamm, Inra, Montpellier

SupAgro, Université de Montpellier, UMR-System, Montpellier, France bruno.rapidel@cirad.fr

\section{Sibelet Nicole}

Cirad, Inra, Montpellier SupAgro,

Université de Montpellier, UMR-Innovation, Montpellier, France; Catie, 7170 Turrialba, Costa Rica

nicole.sibelet@cirad.fr

\section{Chapitre 5}

\section{Bihina M. Armelle}

Université Yaoundé I, Joseph Tchooungui

Akoa, Yaoundé, Cameroun

armelle.bihina@gmail.com

\section{Blanchet A.}

Montpellier SupAgro, Inra, Cirad, Université de Montpellier, Montpellier, France.

liline_34_w@hotmail.fr

\section{Carrière Stéphanie}

IRD, Université Paul Valéry, UMR-Gred, Montpellier, France

stephanie.carriere@ird.fr

\section{Levang Patrice}

IRD, Université Paul Valéry, UMR-Gred, Montpellier, France patrice.levang@ird.fr

\section{Manga Essouma F.}

Irad, Station de Nkolbisson, Yaoundé,

Cameroun

essouma2003@yahoo.fr

\section{Michel Isabelle}

Cirad, Inra, Montpellier SupAgro,

Université de Montpellier, UMR-Innovation,

Montpellier, France

isabelle.michel@supagro.fr

\section{Moisy Christophe}

Cirad, Inra, Montpellier SupAgro,

Université de Montpellier, UMR-Innovation,

Montpellier, France

christophe.moisy@inra.fr

Ngono Francesca

Université Yaoundé I, Joseph Tchooungui

Akoa, Yaoundé, Cameroun

franchescangono@gmail.com

\section{Chapitre 6}

\section{Bouambi Emmanuel}

Irad, département des plantes stimulantes,

Yaoundé, Cameroun

\section{Essobo Jean-Daniel}

Cirad, Ciheam-Iamm, Inra, Montpellier

SupAgro, Université de Montpellier, UMR-System, Montpellier, France 


\section{Jagoret Patrick}

Cirad, Ciheam-Iamm, Inra, Montpellier SupAgro, Université de Montpellier, UMR-System, Montpellier, France patrick.jagoret@cirad.fr

\section{Mvondo Sakouma Kenneth}

Cirad, Ciheam-Iamm, Inra, Montpellier SupAgro, Université de Montpellier, UMR-System, Montpellier, France

\section{Saj Stéphane}

Cirad, Ciheam-Iamm, Inra, Montpellier SupAgro, Université de Montpellier, UMR-System, Montpellier, France; Irad, Département des plantes stimulantes, Yaoundé, Cameroun stephane.saj@cirad.fr

\section{Todem-Ngogue Hervé}

Irad, département des plantes stimulantes, Yaoundé, Cameroun

\section{Chapitre 7}

\section{Gidoin Cynthia}

Inra, UR-PSH, Avignon, France

gidoin.cynthia@gmail.com

\section{Ngo Bieng Marie-Ange}

Cirad, Université de Montpellier, UR-Forêts et sociétés, Montpellier, France; Catie, Programa bosques, biodiversidad y cambio climatico, Turrialba, Costa Rica marie-ange.ngo_bieng@cirad.fr

\section{Ten Hoopen Gerben-Martijn}

Cirad, Université de Montpellier,

UPR-Bioagresseurs, France; Irad, Yaoundé, Cameroun; Cocoa research center, St-Augustine, Trinidad and Tobago gerben-martijn.ten_hoopen@cirad.fr, tenhoopen@cirad.fr

\section{Chapitre 8}

\section{Bidou Jean-Étienne \\ LAM}

jean-etienne.bidou@orange.fr

\section{Droy Isabelle}

IRD, UMI-Résiliences, Bondy, France isabelle.droy@ird.fr

\section{Koukpéré Abidine}

koussouab@gmail.com

\section{Chapitre 9}

Awessou Kohomlan G. Beranger

Direction générale des Eaux, Forêts et Chasse (DGEFC), BP 393 Cotonou, Bénin

kohomlan@yahoo.fr

\section{Peugeot Christophe}

IRD, Université Montpellier,

UMR-HydroSciences, Montpellier,

Montpellier, France

christophe.peugeot@ird.fr

\author{
Agbossou Euloge \\ INE et LHME/FSA, Université Abomey \\ Calavi de Cotonou, PO Box 01 BP 526 \\ Cotonou, Bénin \\ agbossou.euloge@yahoo.fr
}

\section{Seghieri Josiane}

IRD, Université de Montpellier, UMR-HydroSciences, Montpellier, France josiane.seghieri@ird.fr

\section{Chapitre 10}

\section{Clermont-Dauphin Cathy}

Cirad, IRD, Université Montpellier, UMR-Eco\&Sols, Montpellier, France;

Laboratoire mixte international intensification écologique des sols cultivés en Afrique de l'Ouest (LMI Iesol), Campus IRD/Isra

Bel-Air, Dakar, Sénégal

cathy.clermont@ird.fr

\section{Séguis Luc}

IRD, Université de Montpellier, UMR-HydroSciences, Montpellier, France luc.seguis@umontpellier.fr, Luc.Seguis@univ-montp2.fr

\section{Velluet Cécile}

IRD, Université de Montpellier, UMR-HydroSciences, Montpellier, France cecile.velluet@gmail.com, cecile.velluet@inra.fr

\section{Degbé Marc Erudy}

Université Abomey Calavi de Cotonou, PO

Box 01 BP 526 Cotonou, Bénin merudyp3@gmail.com

\section{Cournac Laurent}

Cirad, IRD, Université Montpellier, UMR-Eco\&Sols, Montpellier, France; Laboratoire mixte international intensification écologique des sols cultivés en Afrique de l'Ouest (LMI Iesol), Campus IRD/Isra Bel-Air, Dakar, Sénégal laurent.cournac@cirad.fr 


\section{Seghieri Josiane}

IRD, Université de Montpellier, UMR-HydroSciences, Montpellier, France josiane.seghieri@ird.fr

\section{Chapitre 11}

\section{Sanogo Diaminatou}

Centre national de recherches forestières,

Dakar, Sénégal.

sdiami@yahoo.fr.

\section{Camara Baba Ansoumana}

Laboratoire d'agroforesterie et d'écologie, département d'agroforesterie, UFR-ST Université Assane Seck de Ziguinchor, Sénégal

\section{Diatta Youssouf}

Laboratoire d'agroforesterie et d'écologie, Département d'agroforesterie, UFR-ST Université Assane Seck de Ziguinchor, Sénégal

\section{Coly Lamine}

Laboratoire d'agroforesterie et d'écologie, département d'agroforesterie, UFR-ST Université Assane Seck de Ziguinchor, Sénégal

\section{Diop Mouhamadou}

National de recherches forestières,

Dakar, Sénégal

mamadou77.diop@ucad.edu.sn

\section{Badji Marcel}

Laboratoire d'écologie végétale, département de biologie végétale, FST, Université Cheikh Anta Diop, Dakar, Sénégal badjimarcel@yahoo.fr

\section{Binam Joachim Nyemeck}

Centre mondial de l'agroforesterie (Icraf), Bureau régional de l'Afrique de l'Ouest et du Centre-Node Sahel, Bamako, Mali j.binam@cgiar.org

\section{Chapitre 12}

\section{Assigbetse Komi}

Cirad, IRD, Université Montpellier, Montpellier, UMR-Eco\&Sols, France;

Laboratoire mixte international intensification écologique des sols cultivés en Afrique de l'Ouest (LMI Iesol), Campus IRD/Isra Bel-Air, Dakar, Sénégal

Komi.Assigbetse@ird.fr

\section{Badiane Ndour Yacine}

Cirad, IRD, Université Montpellier, Montpellier, UMR-Eco\&Sols, Montpellier, France; Laboratoire mixte international intensification écologique des sols cultivés en Afrique de l'Ouest (LMI Iesol), Campus IRD-Isra Bel-Air, Dakar, Sénégal yacine.ndour@ird.fr

\section{Balaya Roger}

Université de Thiès, École nationale supérieure d'agriculture, département biologie des plantes, Thiès, Sénégal

brotheroger@yahoo.fr

\section{Bogie N.}

University of California, School of natural sciences, Merced (CA), États-Unis nbogie@ucmerced.edu

\section{Bright M.B.}

Ohio State University, Department of plant pathology, Wooster $(\mathrm{OH})$,

School of environment and natural resources, Columbus (OH), États-Unis mbhbright@gmail.com

\section{Chapuis-Lardy Lydie}

Cirad, IRD, Université Montpellier, Montpellier, UMR-Eco\&Sols, Montpellier, France; Laboratoire mixte international intensification écologique des sols cultivés en Afrique de l'Ouest (LMI Iesol), Campus IRD-Isra Bel-Air, Dakar, Sénégal

Lydie.lardy@ird.fr

\section{Cournac Laurent}

Cirad, IRD, Université Montpellier, Montpellier, UMR-Eco\&Sols, Montpellier, France; Laboratoire mixte international intensification écologique des sols cultivés en Afrique de l'Ouest (LMI Iesol), Campus IRD/ Isra Bel-Air, Dakar, Sénégal laurent.cournac@cirad.fr

Debenport $\mathbf{S}$.

Ohio State University, Department of plant pathology, Wooster $(\mathrm{OH})$, États-Unis debenport.2@osu.edu

Delay C.

Ohio State University, School of environment and natural resources, Columbus $(\mathrm{OH})$, États-Unis cdelay89@gmail.com 


\section{Diakhaté Sidy}

Cirad, IRD, Université Montpellier, Montpellier, UMR-Eco\&Sols, Montpellier, France; Laboratoire mixte international intensification écologique des sols cultivés en Afrique de l'Ouest (LMI Iesol), Campus IRD/ Isra Bel-Air, Dakar, Sénégal sidy.diakhate@ird.fr

\section{Dick R.P.}

Ohio State University, School of environment and natural resources, Columbus $(\mathrm{OH})$,

États-Unis

dick.78@osu.edu

\section{Diédhiou Ibrahima}

École nationale supérieure d'agriculture, département de biologie des plantes, Thiès, Sénégal ibrahima_diedhiou@hotmail.com

\section{Founoune-Mboup Hassan}

Isra, Laboratoire national de recherches et des productions végétales, Campus IRD/Isra Bel Air, Dakar, Sénégal

fhassna@yahoo.fr

\section{Ghezzehei T.}

University of California, School of natural sciences, Merced (CA), États-Unis taghezzehei@ucmerced.edu

\section{Jourdan Christophe}

Cirad, IRD, Université Montpellier, Montpellier, UMR-Eco\&Sols, Montpellier, France; Inra, Montpellier SupAgro, UMR-Lisah, France christophe.jourdan@cirad.fr

\section{McSpadden Gardener B.}

Ohio State University, Department of plant pathology, Wooster $(\mathrm{OH})$, États-Unis mcspadden-garden.1@osu.edu

\section{Sambou Donatien M.}

Cirad, IRD, Université Montpellier, Montpellier, UMR-Eco\&Sols, Montpellier, France; Laboratoire mixte international intensification écologique des sols cultivés en Afrique de l'Ouest (LMI Iesol), Campus IRD/ Isra Bel-Air, Dakar, Sénégal donatienmsambou@yahoo.fr

\section{Chapitre 13}

\section{Allies Aubin}

IRD, Université de Montpellier, UMR-HydroSciences, Montpellier, France aubin.allies@umontpellier.fr

\section{Bil-Assanou Issoufou Hassane}

Université Dan Dicko Dankoulodo de Maradi, Faculté d'agronomie et des sciences de l'environnement, département des Sciences et techniques de productions végétales, Maradi, Niger bil-assanou@hotmail.com

\section{Cappelaere Bernard}

IRD, Université de Montpellier, UMR-HydroSciences, Montpellier, France bernard.cappelaere@umontpellier.fr, bernard.cappelaere@ird.fr

\section{Chazarin Jean-Philippe}

IRD, Université de Montpellier, UMR-HydroSciences, Montpellier, France jean-philippe.chazarin@umontpellier.fr

\section{Demarty Jérôme}

IRD, Université de Montpellier, UMR-HydroSciences, Montpellier, France jerome.demarty@ird.fr

\section{Maïnassara Ibrahim \\ IRD, Niamey, Niger ibrahim.mainassara@ird.fr}

\section{Moussa Moumouni Rachida}

Faculté d'agronomie et des sciences de l'environnement, département des Sciences et Techniques de Productions Végétales, Université Dan Dicko Dankoulodo de Maradi, Maradi, Niger

Rachidamoussa15@gmail.com

\section{Ö̈ Monique}

IRD, Université de Montpellier, UMR-HydroSciences, Montpellier, France monique.oi@umontpellier.fr

\section{Seghieri Josiane}

IRD, Université de Montpellier, UMR-HydroSciences, Montpellier, France josiane.seghieri@ird.fr

\section{Velluet Cécile}

IRD, Université de Montpellier, UMR-HydroSciences, Montpellier, France cecile.velluet@gmail.com, cecile.velluet@inra.fr

\section{Chapitre 14}

\author{
Bidou Jean-Étienne \\ Ifree, Villiers-en-Bois, France \\ jebidou@ades.cnrs.fr
}




\section{Droy Isabelle}

IRD, UMI-Résiliences, Bondy, France

isabelle.droy@ird.fr

\section{Freguin-Gresh Sandrine}

Cirad, Inra, Montpellier SupAgro, Université de Montpellier, UMR-Art-Dev, Montpellier, France; UCA, Nitlapan, Managua, Nicaragua sandrine.freguin@cirad.fr

\section{Le Coq Jean-François}

Cirad, Inra, Montpellier SupAgro, Université de Montpellier, UMR-Art-Dev, Montpellier, France; CINPE, UNA, Heredia, Costa Rica jean-francois.le_coq@cirad.fr, JF.LeCoq@ CGIAR.ORG

\section{Pédelahore Philippe}

Cirad, Inra, Montpellier SupAgro, Université de Montpellier, UMR-Innovation, Montpellier, France; Icraf, SD1, P.O. Box 30677-00 100, Nairobi, Kenya philippe.pedelahore@cirad.fr

\section{Sibelet Nicole}

Cirad, Inra, Montpellier SupAgro,

Université de Montpellier, UMR-Innovation, Montpellier, France; Catie, 7170 Turrialba, Costa Rica nicole.sibelet@cirad.fr

\section{Chapitre 15}

\section{Allinne Clémentine \\ Cirad, Ciheam-Iamm, Inra, Montpellier \\ SupAgro, Université de Montpellier, UMR-System, Montpellier, France; Catie, Programma de agricultura, ganaderia y agroforesteria, Turrialba, Costa Rica clementine.allinne@cirad.fr}

\section{Avelino Jacques}

Cirad, Université de Montpellier, UPR-Bioagresseurs, Montpellier, France; Catie, Programma de agricultura, ganaderia y agroforesteria, Turrialba, Costa Rica jacques.avelino@cirad.fr

\section{Babin Régis}

Cirad, Université de Montpellier, UPR-Bioagresseurs, France; International centre of insect physiology and ecology, Nairobi, Kenya regis.babin@cirad.fr

\section{Bagny Beilhe Leila}

Cirad, Université de Montpellier, UPR-Bioagresseurs, France; Irad, Yaoundé, Cameroun; Catie, Programma de agricultura, ganaderia y agroforesteria, Turrialba,

Costa Rica

leila.bagny@cirad.fr

\section{Brévault Thierry}

Cirad, Université de Montpellier, UR-Aïda, Montpellier, France; Biopass, Isra-IRD-UcadCirad, Dakar, Sénégal thierry.brevault@cirad.fr

\section{Gidoin Cynthia}

Cirad, Ciheam-Iamm, Inra, Montpellier SupAgro, Université de Montpellier, UMR-System, Montpellier, France; Inra, UR-PSH, Avignon, France cindy.gidoin@avignon.inra.fr

\section{Motisi Natacha}

Cirad, Université de Montpellier, UPR-Bioagresseurs, Montpellier, France;

Catie, Programma de agricultura, ganaderia y agroforesteria, Turrialba, Costa Rica natacha.motisi@cirad.fr

\section{Ngo Bieng Marie-Ange}

Cirad, Université de Montpellier, UR-Forêts et sociétés, France; Catie, Programa bosques, biodiversidad y cambio climatico, Turrialba, Costa Rica

marie-ange.ngo_bieng@cirad.fr

\section{Soti Valérie}

Cirad, Université de Montpellier, UR-Aïda, Montpellier, France; CSE, Centre de suivi écologique, Dakar, Sénégal valerie.soti@cirad.fr

\section{Ten Hoopen Gerben-Martijn}

Cirad, Université de Montpellier, UPR-Bioagresseurs, France; Irad, Yaoundé, Cameroun; Cocoa research center, St-Augustine, Trinidad and Tobago gerben-martijn.ten_hoopen@cirad.fr 